\title{
Unsaturated Vicinal Frustrated Lewis Pair Formation by Electrocyclic Ring Closure and Their Reaction with Nitric Oxide
}

Thomas Özgün, ${ }^{\S}$ Guo-Qiang Chen, ${ }^{\S}$ Constantin G. Daniliuc, ${ }^{\S}$ Alison C. McQuilken, ${ }^{\dagger}$ Timothy H. Warren, ${ }^{\dagger}$ Robert Knitsch, ${ }^{\ddagger}$ Hellmut Eckert, ${ }^{\ddagger}$ Gerald Kehr, ${ }^{\S}$ Gerhard Erker ${ }^{\star \S}$

§ Organisch-Chemisches Institut, Westfälische Wilhelms-Universität, Corrensstrasse 40, 48149 Münster, Germany

† Department of Chemistry, Georgetown University, Box 571227, Washington, D.C. 20057-1227, United States

‡ Institut für Physikalische Chemie, Westfälische Wilhelms-Universität, Corrensstrasse 30, 48149 Münster, Germany

\section{Supporting Information}




\section{Table of contents}

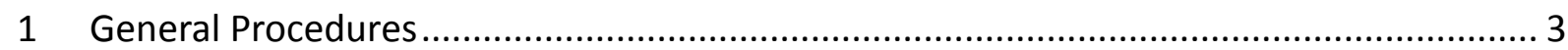

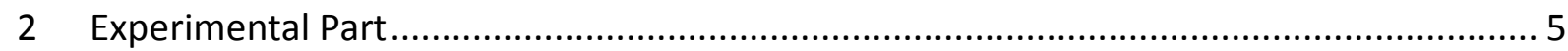

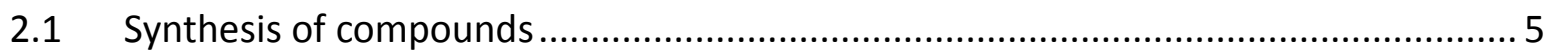

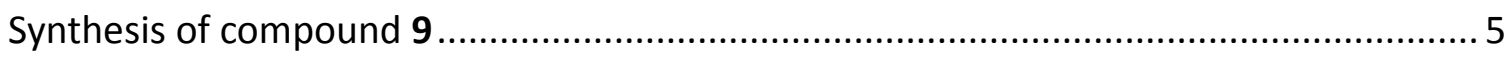

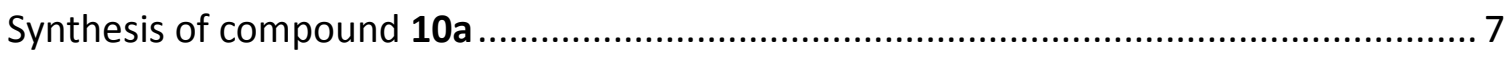

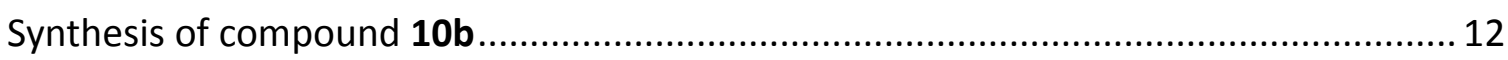

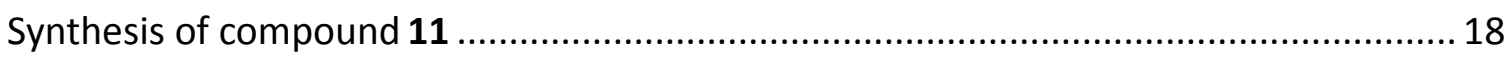

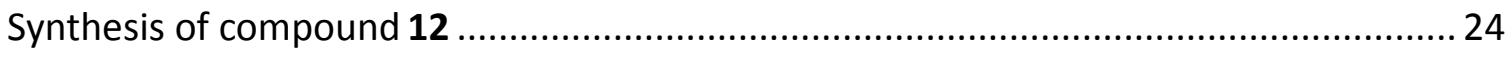

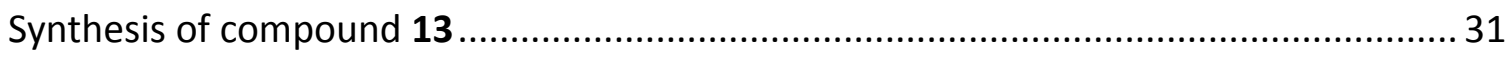

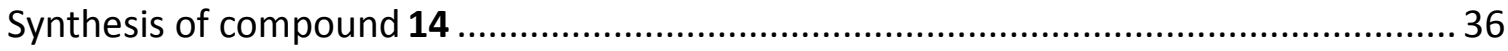

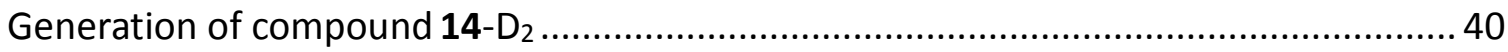

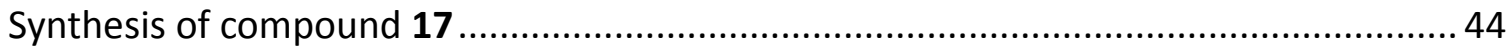

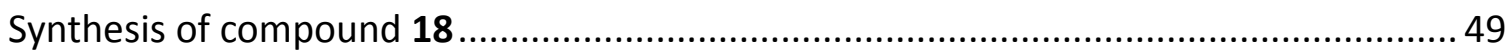

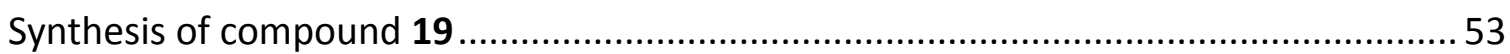

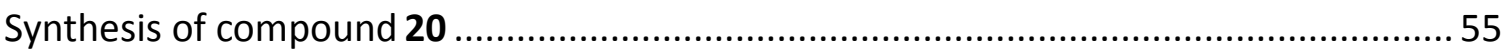

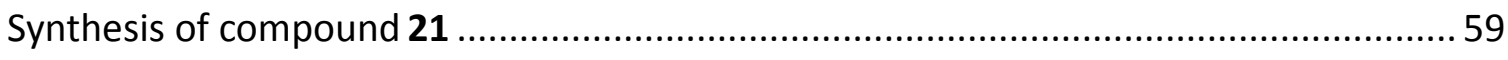

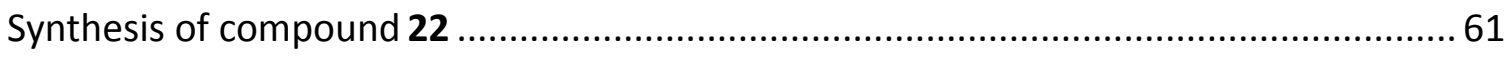

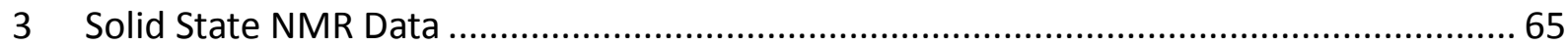

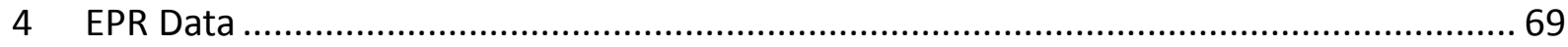




\section{General Procedures}

All syntheses involving air- and moisture sensitive compounds were carried out using standard Schlenk-type glassware (or in a glove box) under an atmosphere of argon. Solvents were dried and stored under an argon atmosphere. NMR spectra were recorded on an Agilent DD2-500 $\mathrm{MHz}\left({ }^{1} \mathrm{H}: 500 \mathrm{MHz},{ }^{13} \mathrm{C}: 126 \mathrm{MHz},{ }^{19} \mathrm{~F}: 470 \mathrm{MHz},{ }^{11} \mathrm{~B}: 160 \mathrm{MHz},{ }^{31} \mathrm{P}: 202 \mathrm{MHz}\right)$ and on an Agilent DD2- $600 \mathrm{MHz}\left({ }^{1} \mathrm{H}: 600 \mathrm{MHz},{ }^{13} \mathrm{C}: 151 \mathrm{MHz},{ }^{19} \mathrm{~F}: 564 \mathrm{MHz},{ }^{11} \mathrm{~B}: 192 \mathrm{MHz},{ }^{31} \mathrm{P}: 243\right.$ $\mathrm{MHz}) .{ }^{1} \mathrm{H}$ NMR and ${ }^{13} \mathrm{C}$ NMR: chemical shifts are given relative to TMS and referenced to the solvent signal. ${ }^{19} \mathrm{~F} N M R$ : chemical shifts are given relative to $\mathrm{CFCl}_{3}(\delta=0$, external reference), ${ }^{11} \mathrm{~B}$ NMR: chemical shifts are given relative to $\mathrm{BF}_{3} \cdot \mathrm{Et}_{2} \mathrm{O}\left(\delta=0\right.$, external reference), ${ }^{31} \mathrm{P}$ NMR: chemical shifts are given relative to $\mathrm{H}_{3} \mathrm{PO}_{4}\left(85 \%\right.$ in $\left.\mathrm{D}_{2} \mathrm{O}\right)(\delta=0$, external reference). NMR assignments were supported by additional 2D NMR experiments. Elemental analyses were performed on an Elementar Vario EI III. IR spectra were recorded on a Varian 3100 FT-IR (Excalibur Series). Melting points and decomposition points were obtained with a DSC 2010 (TA Instruments). HRMS was recorded on GTC Waters Micromass (Manchester, UK).

X-Ray diffraction: For compounds 10a, 10b, 12, 14, 19, 21 and 22 data sets were collected with a Nonius Kappa CCD diffractometer. Programs used: data collection, COLLECT (R. W. W. Hooft, Bruker AXS, 2008, Delft, The Netherlands); data reduction Denzo-SMN (Z. Otwinowski, W. Minor, Methods Enzymol. 1997, 276, 307-326); absorption correction, Denzo (Z. Otwinowski, D. Borek, W. Majewski, W. Minor, Acta Crystallogr. 2003, A59, 228-234); structure solution SHELXS-97 (G. M. Sheldrick, Acta Crystallogr. 1990, A46, 467-473); structure refinement SHELXL-97 (G. M. Sheldrick, Acta Crystallogr. 2008, A64, 112-122) and graphics, XP (BrukerAXS, 2000). For compound 18 and 20 data sets were collected with a Kappa CCD APEXII Bruker diffractometer. For compounds 11, 17, 20' data sets were collected with a D8 Venture Dual Source 100 CMOS diffractometer. Programs used: data collection: APEX2 V2014.5-0 (Bruker AXS Inc., 2014); cell refinement: SAINT V8.34A (Bruker AXS Inc., 2013); data reduction: SAINT V8.34A (Bruker AXS Inc., 2013); absorption correction, SADABS V2014/2 (Bruker AXS Inc., 2014); structure solution SHELXT-2014 (Sheldrick, 2014); structure refinement SHELXL-2014 (Sheldrick, 2014) and graphics, XP (Bruker AXS Inc., 2014). $R$ values are given for observed reflections, and $w R^{2}$ values are given for all reflections. Exceptions and special features: For compound 11 one pentane molecule, for compound 14 one dichloromethane molecule, for compounds 19 and 20' two dichloromethane molecules and for compound $\mathbf{2 1}$ one phenyl group and one part of the six membered ring C5 to C10 are disordered over two positions. Several restraints (SADI, SAME, ISOR and SIMU) were used in order to improve refinement stability. For compounds 10a and 12 one badly pentane molecule and for compounds $\mathbf{1 7}$ and $\mathbf{1 8}$ one badly half pentane molecule were found in the asymmetric unit and could not be satisfactorily refined. The program SQUEEZE (A. L. Spek J. 
Appl. Cryst., 2003, 36, 7-13) was therefore used to remove mathematically the effect of the solvent. The quoted formula and derived parameters are not included the squeezed solvent molecules. Compound $\mathbf{1 0 b}$ presents one tBu group and one six membered ring at $\mathrm{C} 1$ atom disordered over two positions. Several restraints (SADI, SAME, ISOR and SIMU) were used in order to improve refinement stability. Moreover, one badly disordered pentane molecule was found in the asymmetrical unit and could not be satisfactorily refined. The program SQUEEZE was therefore used to remove mathematically the effect of the solvent. The quoted formula and derived parameters are not included the squeezed solvent molecule.

Materials: Vinylboranes 6a, 6b [Ekkert, O.; Tuschewitzki, O.; Daniliuc, C. G.; Kehr, G.; Erker, G. Chem. Commun., 2013, 49, 6992-6994; Parks, D. J.; Piers, W. E.; Yap, G. P. A. Organometallics, 1998, 17, 5492-5503.] were prepared according to the literature. 


\section{Experimental Part}

\subsection{Synthesis of compounds}

\section{Synthesis of compound 9}

For analogous synthesis of the diphenylphospane derivative see: Chen, G.-Q.; Kehr, G.;

Daniliuc, C. G.; Erker, G. Org. Biomol. Chem. 2015, 13, 765-769.

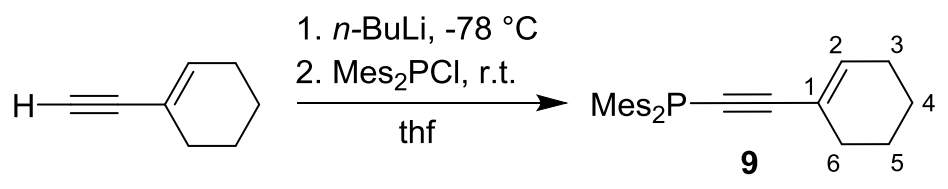

Scheme S1

$n$-Butyllithium $(10.0 \mathrm{~mL}, 16.0 \mathrm{mmol}, 1.0 \mathrm{eq})$ was added dropwise to a solution of 1 ethynylcyclohexene $(1.9 \mathrm{~mL}, 1.7 \mathrm{~g}, 16.0 \mathrm{mmol}, 1.0 \mathrm{eq})$ in tetrahydrofuran $(80 \mathrm{~mL})$ at $-78^{\circ} \mathrm{C}$. The reaction mixture was warmed to $r$. t. and stirred for $10 \mathrm{~min}$. Then a solution of chlorodimesitylphosphane $(4.9 \mathrm{~g}, 16.0 \mathrm{mmol}, 1.0 \mathrm{eq})$ in tetrahydrofuran $(30 \mathrm{~mL})$ was added dropwise to the obtained dark brown suspension at $-78^{\circ} \mathrm{C}$. The reaction mixture was stirred for $10 \mathrm{~min}$ at $-78{ }^{\circ} \mathrm{C}$ and subsequently stirred for 2 hours at ambient temperature. Then all volatiles were removed in vacuo and the sticky residue was suspended in pentane $(100 \mathrm{~mL})$. The resulting suspension was filtered via cannula (WHATMAN glass fiber filter) and all volatiles were removed from the filtrate in vacuo to give a brown oil. After Purification via column chromatography (silica: $\mathrm{CH}_{2} \mathrm{Cl}_{2}: \mathrm{CyH}=15: 85 ; \mathrm{R}_{\mathrm{f}}$ : 0.70 ) compound 9 was obtained as a colorless, crystalline solid $(5.1 \mathrm{~g}, 13.6 \mathrm{mmol}, 85 \%)$.

IR $(\mathrm{KBr}): \tilde{v}\left[\mathrm{~cm}^{-1}\right]=3063(\mathrm{w}), 2958(\mathrm{w}), 2855(\mathrm{w}), 2727(\mathrm{w}), 2661(\mathrm{w}), 2466(\mathrm{w}), 2300(\mathrm{w}), 2133$ (m), 1908 (w), $1881(w), 1720(w), 1601(m), 1553(w), 1465(m), 1433(s), 1407(m), 1372$ (m), $1346(w), 1074(w), 1028(m), 996(w), 917(m), 845(s), 787(m), 712(w), 689(m), 616$ (m), $600(\mathrm{~m}), 558(\mathrm{~s}), 460(\mathrm{w}), 431(\mathrm{w})$.

M.p. $80^{\circ} \mathrm{C}$.

Anal. Calc. for $\mathrm{C}_{26} \mathrm{H}_{31} \mathrm{P}: \mathrm{C}: 83.39$; H: 8.34. Found: C: 83.20; $\mathrm{H}: 8.43$.

${ }^{1} \mathrm{H}$ NMR $\left(500 \mathrm{MHz}\right.$, dichloromethane- $\left.d_{2}, 299 \mathrm{~K}\right): \delta 6.81\left(\mathrm{dm},{ }^{4} \mathrm{~J}_{\mathrm{PH}}=3.2 \mathrm{~Hz}, 4 \mathrm{H}, m-\mathrm{Mes}\right), 6.08$ (m, 1H, 2-CH), 2.37 (s, 12H, o-Mes), 2.24 (s, 6H, p-Mes), $2.11\left(\mathrm{~m}, 2 \mathrm{H}, 6-\mathrm{CH}_{2}\right), 2.09(\mathrm{~m}, 2 \mathrm{H}, 3-$ $\left.\mathrm{CH}_{2}\right), 1.62\left(\mathrm{~m}, 2 \mathrm{H}, 5-\mathrm{CH}_{2}\right), 1.57\left(\mathrm{~m}, 2 \mathrm{H}, 4-\mathrm{CH}_{2}\right)$.

${ }^{13} \mathrm{C}\left\{{ }^{1} \mathrm{H}\right\}$ NMR (126 MHz, dichloromethane- $\left.d_{2}, 299 \mathrm{~K}\right): \delta 142.2\left(\mathrm{~d},{ }^{2} \mathrm{JPC}_{\mathrm{PC}}=15.6 \mathrm{~Hz}, \mathrm{o}-\mathrm{Mes}\right), 138.7$ (p-Mes), $135.6\left(\mathrm{~d},{ }^{4} J_{\mathrm{PC}}=2.6 \mathrm{~Hz}, 2-\mathrm{CH}\right), 130.5$ (d, $\left.{ }^{1} J_{\mathrm{PC}}=12.6 \mathrm{~Hz}, i-\mathrm{Mes}\right), 130.2\left(\mathrm{~d},{ }^{3} J_{\mathrm{PC}}=\right.$ $3.6 \mathrm{~Hz}, m-\mathrm{Mes}), 121.7\left(\mathrm{~d},{ }^{3} \mathrm{JPC}_{\mathrm{PC}}=1.5 \mathrm{~Hz}, 1-\mathrm{C}=\right), 109.2$ (d, $\left.{ }^{2} \mathrm{JPC}_{\mathrm{PC}}=8.8 \mathrm{~Hz}, \equiv \mathrm{C}\right), 84.3\left(\mathrm{~d},{ }^{1} \mathrm{~J}_{\mathrm{PC}}=\right.$ $3.4 \mathrm{~Hz}, \mathrm{PC} \equiv), 28.8\left(\mathrm{~d},{ }^{4} \mathrm{JPC}_{\mathrm{PC}}=1.6 \mathrm{~Hz}, 6-\mathrm{CH}_{2}\right), 26.1\left(3-\mathrm{CH}_{2}\right), 23.0\left(\mathrm{~d},{ }^{3} \mathrm{~J}_{\mathrm{PC}}=14.4 \mathrm{~Hz}, o-\mathrm{CH}_{3}{ }^{\mathrm{Mes}}\right)$, $22.6\left(5-\mathrm{CH}_{2}\right), 21.8\left(4-\mathrm{CH}_{2}\right), 21.0\left(p-\mathrm{CH}_{3}{ }^{\mathrm{Mes}}\right)$. 
${ }^{31} \mathbf{P}\left\{{ }^{1} \mathrm{H}\right\}$ NMR $\left(202 \mathrm{MHz}\right.$, dichloromethane- $\left.d_{2}, 299 \mathrm{~K}\right): \delta-56.5\left(v_{1 / 2} \sim 3 \mathrm{~Hz}\right)$.
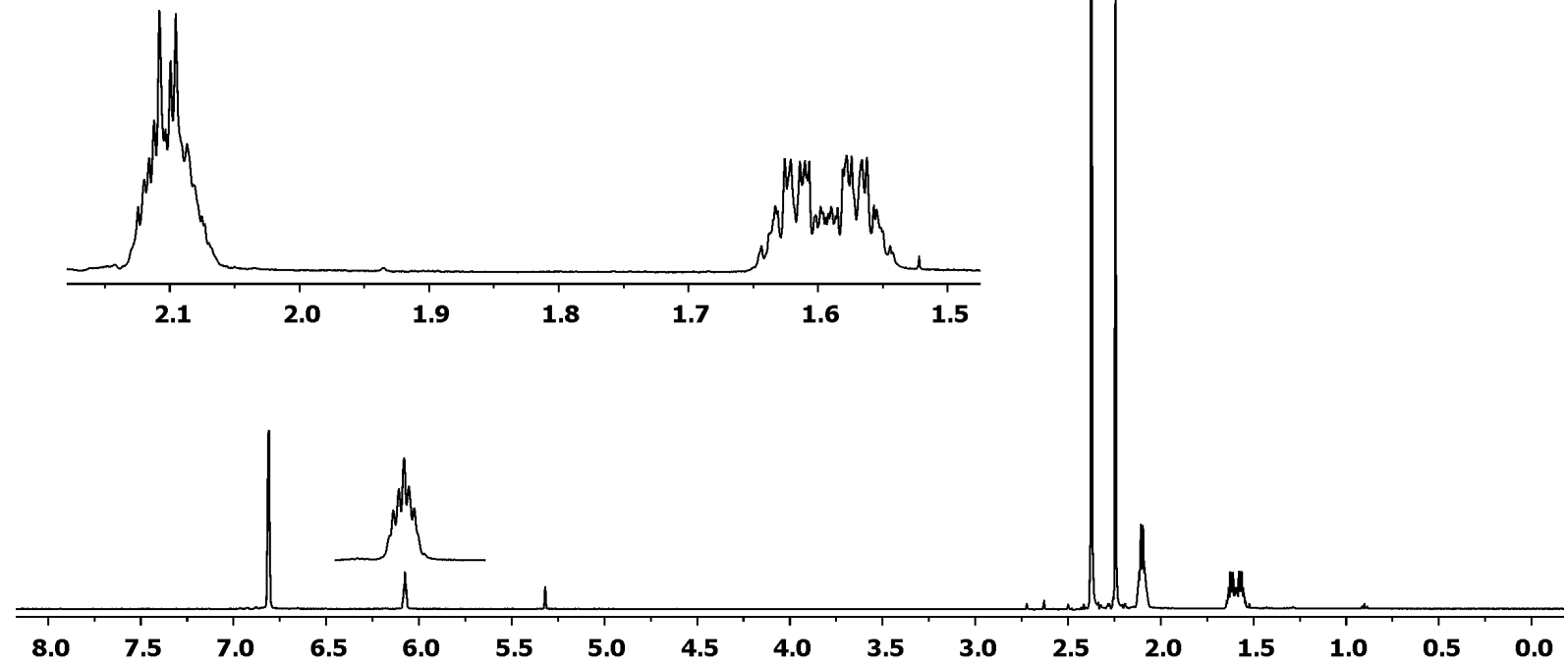

Figure S1: ${ }^{1} \mathrm{H}$ NMR $\left(500 \mathrm{MHz}\right.$, dichloromethane- $\left.d_{2}, 299 \mathrm{~K}\right)$ spectrum of compound 9
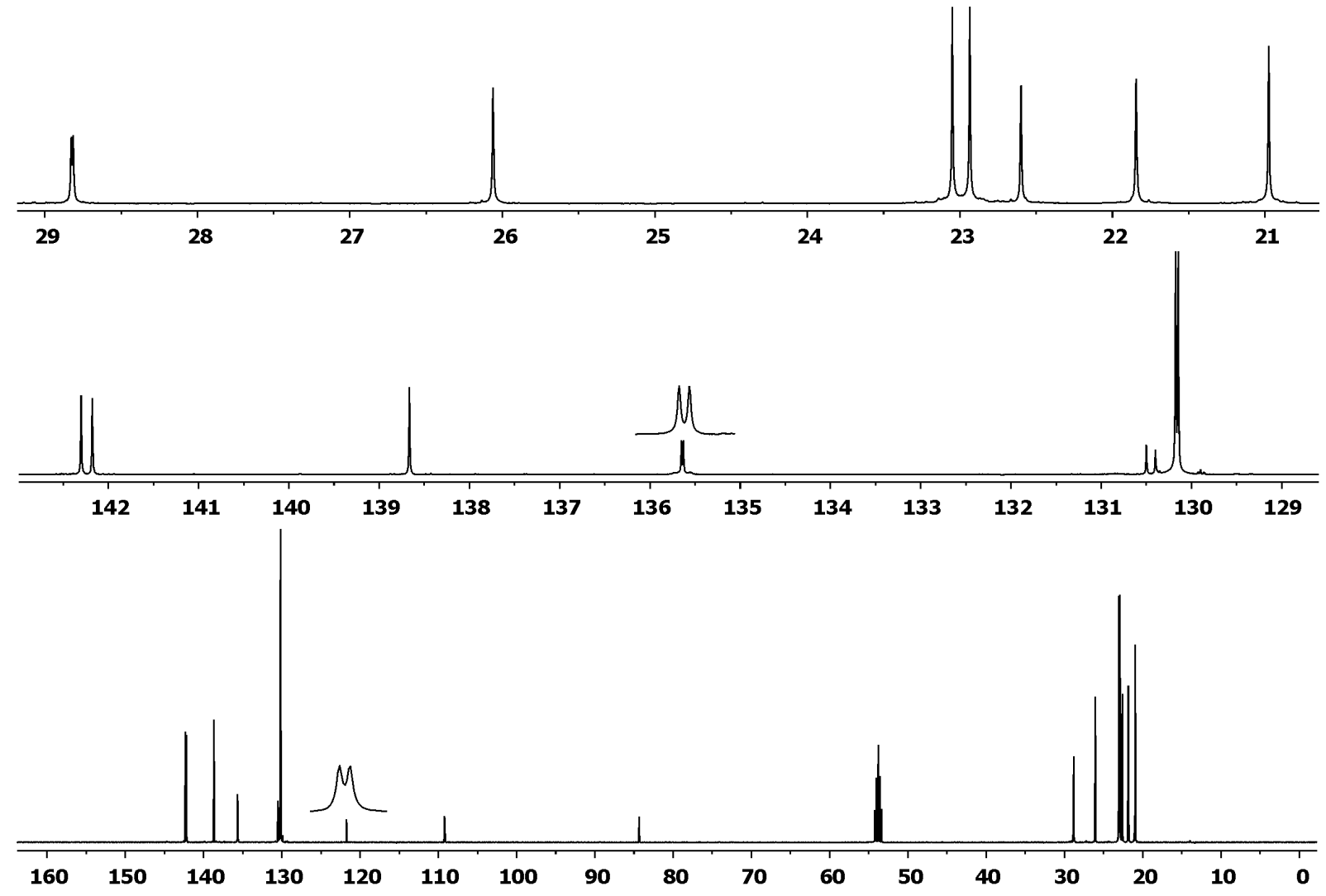

Figure S2: ${ }^{13} \mathrm{C}\left\{{ }^{1} \mathrm{H}\right\}$ NMR $(126 \mathrm{MHz}$, dichloromethane-d $2,299 \mathrm{~K})$ spectrum of compound 9 


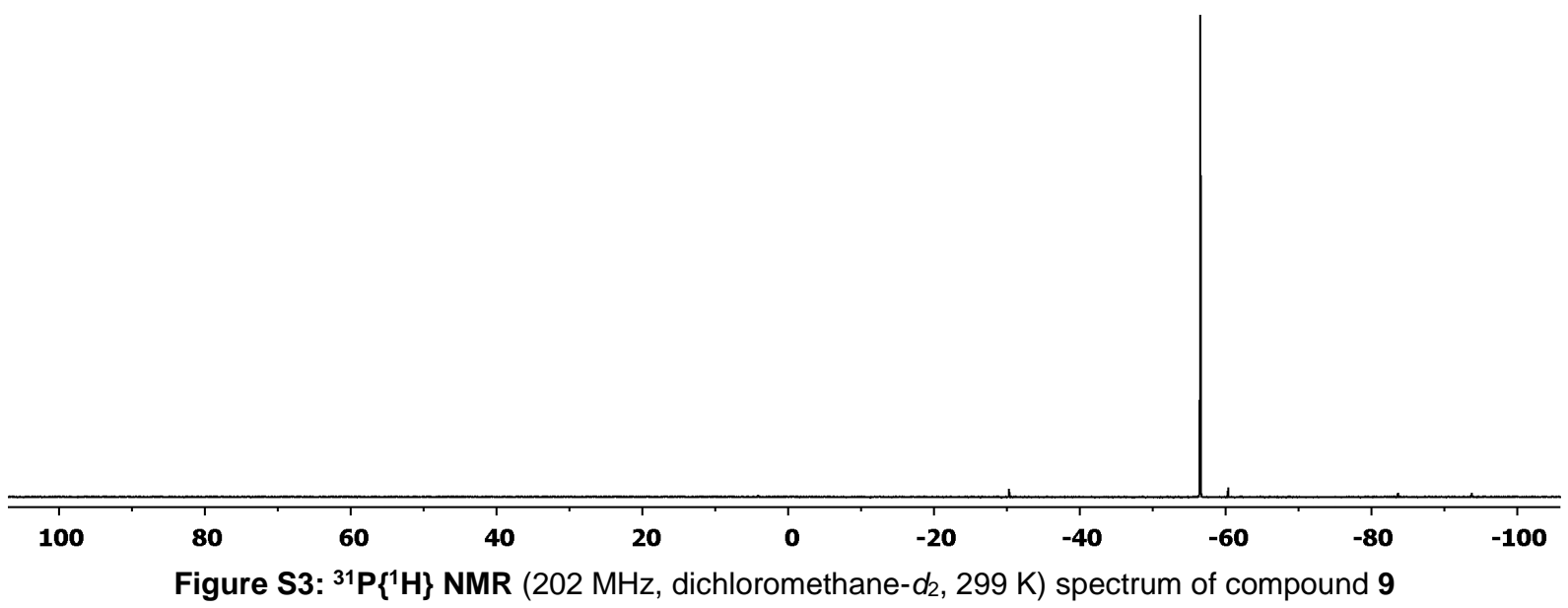

\section{Synthesis of compound $10 a$}

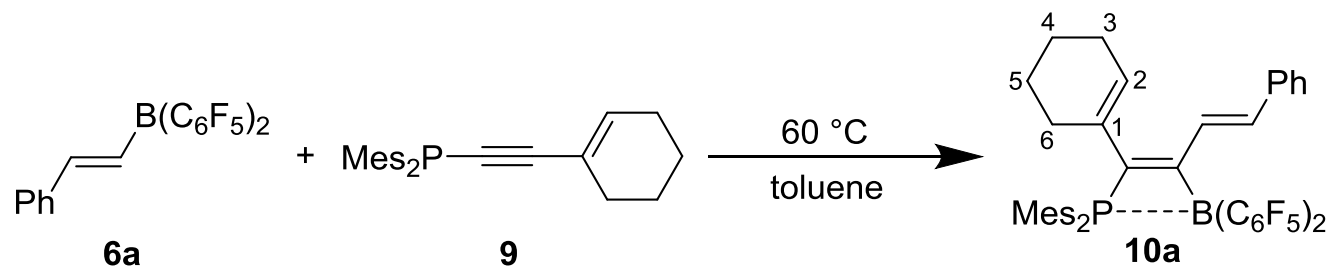

Scheme S2

A solution of phosphane $9(417.6 \mathrm{mg}, 1.1 \mathrm{mmol}, 1.0 \mathrm{eq})$ in toluene $(6 \mathrm{~mL})$ was added to a solution of borane $6 \mathbf{a}(500.0 \mathrm{mg}, 1.1 \mathrm{mmol}, 1.0 \mathrm{eq})$ in toluene $(4 \mathrm{~mL})$. The reaction mixture was stirred at $60^{\circ} \mathrm{C}$ for $3 \mathrm{~d}$ and then all volatiles were removed in vacuo. The resulting sticky residue was dissolved in pentane $(3 \mathrm{~mL})$, which was subsequently removed in vacuo. The obtained solid was washed with pentane $(5 \times 3 \mathrm{~mL})$ to give compound 10a as a white powdery solid (822.0 mg, $1.0 \mathrm{mmol}, 90 \%)$.

IR $(\mathrm{KBr}): \tilde{v}\left[\mathrm{~cm}^{-1}\right]=3696(\mathrm{w}), 3023(\mathrm{~m}), 2929(\mathrm{~s}), 2856(\mathrm{~m}), 2347(\mathrm{w}), 1947(\mathrm{w}), 1723(\mathrm{w}), 1642$ (s), 1604 (s), 1553 (w), 1515 (s), 1455 (m), $1380(\mathrm{~m}), 1341$ (w), 1287 (s), 1269 (m), $1248(\mathrm{~m})$, $1152(\mathrm{w}), 1091$ (s), $1034(\mathrm{~m}), 1024$ (w), 996 (s), 852 (s), 805 (m), 773 (m), 741 (s), 696 (s), $643(\mathrm{~m}), 629(\mathrm{~m}), 607(\mathrm{~m}), 575(\mathrm{~m}), 553(\mathrm{~m}), 499(\mathrm{~m}), 453(\mathrm{w}), 418(\mathrm{~m})$.

Decomp. $200{ }^{\circ} \mathrm{C}$.

Anal. Calc. for $\mathrm{C}_{46} \mathrm{H}_{38} \mathrm{BF}_{10} \mathrm{P}: \mathrm{C}: 67.17 ; \mathrm{H}: 4.66$. Found: $\mathrm{C}: 66.78 ; \mathrm{H}: 4.60$.

${ }^{1} \mathrm{H}$ NMR (600 MHz, dichloromethane- $\left.d_{2}, 213 \mathrm{~K}\right): \delta 7.28(\mathrm{~m}, 2 \mathrm{H}, \mathrm{o}-\mathrm{Ph}), 7.23(\mathrm{~m}, 2 \mathrm{H}, m-\mathrm{Ph})$, $7.18(\mathrm{~m}, 1 \mathrm{H}, \mathrm{p}-\mathrm{Ph}), 7.14\left(\mathrm{br} \mathrm{d},{ }^{3} \mathrm{JHH}_{\mathrm{HH}}=16.3 \mathrm{~Hz}, 1 \mathrm{H},=\mathrm{CH}\right), 6.97\left(\mathrm{~d},{ }^{4} J_{\mathrm{PH}}=3.5 \mathrm{~Hz}, 1 \mathrm{H}, m-\right.$ Mes $\left.^{\mathrm{a}}\right), 6.81\left(\mathrm{~s}, 1 \mathrm{H}, \mathrm{m}^{\prime}-\mathrm{Mes}^{\mathrm{a}}\right), 6.79\left(\mathrm{~m}, 1 \mathrm{H}, m-\mathrm{Mes}^{\mathrm{b}}\right), 6.40\left(\mathrm{~d},{ }^{3} \mathrm{~J}_{\mathrm{HH}}=16.3 \mathrm{~Hz}, 1 \mathrm{H},=\mathrm{CH}^{\mathrm{Ph}}\right), 6.38$ $\left(\mathrm{d},{ }^{4} J_{\mathrm{PH}}=2.5 \mathrm{~Hz}, 1 \mathrm{H}, m^{\prime}-\mathrm{Mes}^{\mathrm{b}}\right), 5.87(\mathrm{~s}, 1 \mathrm{H}, 2-\mathrm{CH}), 2.57\left(\mathrm{~s}, 3 \mathrm{H}, \mathrm{o}-\mathrm{CH}_{3}{ }^{\mathrm{Mes}, b}\right), 2.45(\mathrm{~m}, 3 \mathrm{H}, 0-$ $\left.\mathrm{CH}_{3}{ }^{\mathrm{Mes}, \mathrm{a}}\right), 2.30,2.20$ (each m, each 1H, 3- $\left.\mathrm{CH}_{2}\right)^{\mathrm{t}}, 2.23\left(\mathrm{~s}, 3 \mathrm{H}, p-\mathrm{CH}_{3}{ }^{\mathrm{Mes}, \mathrm{a}}\right), 2.20\left(\mathrm{~s}, 3 \mathrm{H}, o^{\prime}-\right.$ 
$\mathrm{CH}_{3}{ }^{\text {Mes,a }}$ ), 2.10 (s, 3H, $p-\mathrm{CH}_{3}{ }^{\text {Mes,b }}$ ), 1.77, 1.70 (each m, each $\left.1 \mathrm{H}, 5-\mathrm{CH}_{2}\right)^{\mathrm{t}}, 1.65,1.52$ (each m, each $\left.1 \mathrm{H}, 4-\mathrm{CH}_{2}\right)^{\mathrm{t}}, 1.61\left(\mathrm{~s}, 3 \mathrm{H}, \mathrm{o}^{\prime}-\mathrm{CH}_{3}{ }^{\mathrm{Mes}, \mathrm{b}}\right), 1.58,1.43$ (each $\mathrm{m}$, each $\left.1 \mathrm{H}, 6-\mathrm{CH}_{2}\right)^{\mathrm{t}}$. ${ }^{\mathrm{t}}$ tentatively assigned]

${ }^{13} \mathrm{C}\left\{{ }^{1} \mathrm{H}\right\}$ NMR $\left(151 \mathrm{MHz}\right.$, dichloromethane- $\left.d_{2}, 213 \mathrm{~K}\right): \delta 166.3\left(\mathrm{~d},{ }^{2} \mathrm{JPC}_{\mathrm{PC}}=29.6 \mathrm{~Hz}, \mathrm{BC}=\right), 143.1$ $\left(\mathrm{d},{ }^{2} \mathrm{JPC}_{\mathrm{PC}}=17.8 \mathrm{~Hz}, \mathrm{o}-\mathrm{Mes}^{\mathrm{a}}\right), 143.0\left(o^{\prime}-\mathrm{Mes}^{\mathrm{a}}\right), 142.5\left(\mathrm{~d},{ }^{2} \mathrm{JPC}_{\mathrm{PC}}=3.4 \mathrm{~Hz}, \mathrm{o}-\mathrm{Mes}^{\mathrm{b}}\right), 141.3\left(\mathrm{~d},{ }^{4} \mathrm{~J}_{\mathrm{PC}}=\right.$ $\left.2.4 \mathrm{~Hz}, p-M_{e s}^{\mathrm{a}}\right), 139.9\left(p-\mathrm{Mes}^{\mathrm{b}}\right), 139.8\left(\mathrm{~d},{ }^{2} \mathrm{JPC}_{\mathrm{PC}}=10.2 \mathrm{~Hz}, \mathrm{o}^{\prime}-\mathrm{Mes}^{\mathrm{b}}\right), 138.7\left(\mathrm{~d},{ }^{1} \mathrm{~J}_{\mathrm{PC}}=50.6 \mathrm{~Hz}\right.$, $\mathrm{PC}=), 136.3\left(=\mathrm{CH}^{\mathrm{Ph}}\right), 136.1(i-\mathrm{Ph}), 133.2\left(\mathrm{~d},{ }^{2} \mathrm{~J}_{\mathrm{PC}}=3.3 \mathrm{~Hz}, 1-\mathrm{C}=\right), 130.2\left(\mathrm{~d},{ }^{3} \mathrm{JPC}_{\mathrm{PC}}=8.3 \mathrm{~Hz}, \mathrm{~m}-\right.$ $\left.\mathrm{Mes}^{\mathrm{b}}\right), 130.0\left(\mathrm{~d},{ }^{3} \mathrm{JPC}_{\mathrm{PC}}=8.3 \mathrm{~Hz}, 2-\mathrm{CH}\right), 129.6\left(\mathrm{~d},{ }^{3} \mathrm{JPC}_{\mathrm{PC}}=5.9 \mathrm{~Hz}, \mathrm{~m}^{\prime}-\mathrm{Mes}^{\mathrm{a}}\right), 129.3\left(\mathrm{~d},{ }^{3} J_{\mathrm{PC}}=9.2 \mathrm{~Hz}\right.$, $m$-Mes ${ }^{\mathrm{a}}$ ), $129.2\left(\mathrm{~d},{ }^{3} \mathrm{JPC}_{\mathrm{PC}}=9.2 \mathrm{~Hz}, m^{\prime}-\mathrm{Mes}^{\mathrm{b}}\right), 128.3(m-\mathrm{Ph}), 128.1(p-\mathrm{Ph}), 126.6(o-\mathrm{Ph}), 125.4$ $\left(\mathrm{d},{ }^{1} J_{\mathrm{PC}}=25.3 \mathrm{~Hz}, i-\mathrm{Mes}^{\mathrm{a}}\right), 124.6\left(\mathrm{~d},{ }^{1} J_{\mathrm{PC}}=39.9 \mathrm{~Hz}, i-\mathrm{Mes}^{\mathrm{b}}\right), 124.4\left(\mathrm{~d},{ }^{3} J_{\mathrm{PC}}=46.8 \mathrm{~Hz},=\mathrm{CH}\right)$, $27.4\left(5-\mathrm{CH}_{2}\right)^{\mathrm{t}}, 25.3\left(3-\mathrm{CH}_{2}\right)^{\mathrm{t}}, 25.0\left(\mathrm{br} \mathrm{d},{ }^{3} \mathrm{JPC}_{\mathrm{PC}}=13.5 \mathrm{~Hz}, \mathrm{o}_{-} \mathrm{CH}_{3}{ }^{\mathrm{Mes}, \mathrm{b}}\right), 23.7\left(\mathrm{dm}, J=8.7 \mathrm{~Hz}, o^{-}\right.$ $\left.\mathrm{CH}_{3}{ }^{\mathrm{Mes}, \mathrm{a}}\right), 22.4\left(6-\mathrm{CH}_{2}\right)^{\mathrm{t}}, 22.3\left(\mathrm{~d},{ }^{3} J_{\mathrm{PC}}=2.2 \mathrm{~Hz}, o^{\prime}-\mathrm{CH}_{3}{ }^{\mathrm{Mes}, \mathrm{a}}\right), 22.0(\mathrm{dd}, J=9.5 \mathrm{~Hz}, J=3.9 \mathrm{~Hz}$, $\left.o^{\prime}-\mathrm{CH}_{3}{ }^{\mathrm{Mes}, \mathrm{b}}\right), 21.5\left(4-\mathrm{CH}_{2}\right)^{\mathrm{t}}, 20.7\left(p-\mathrm{CH}_{3}{ }^{\mathrm{Mes}, \mathrm{a}}\right), 20.1\left(p-\mathrm{CH}_{3}{ }^{\mathrm{Mes}, \mathrm{b}}\right) .\left[\mathrm{C}_{6} \mathrm{~F}_{5}\right.$ not listed; ${ }^{\mathrm{t}}$ tentatively assigned]

${ }^{31} \mathbf{P}\left\{{ }^{1} \mathrm{H}\right\}$ NMR $\left(243 \mathrm{MHz}\right.$, dichloromethane- $\left.d_{2}, 213 \mathrm{~K}\right): \delta 8.8$ (partial relaxed 1:1:1:1 q, JPB $25 \mathrm{~Hz})$.

${ }^{11} \mathrm{~B}\left\{{ }^{1} \mathrm{H}\right\}$ NMR $\left(192 \mathrm{MHz}\right.$, dichloromethane- $\left.d_{2}, 213 \mathrm{~K}\right): \delta-2.0\left(v_{1 / 2} \sim 1900 \mathrm{~Hz}\right)$

${ }^{19} \mathrm{~F}$ NMR $\left(564 \mathrm{MHz}\right.$, dichloromethane- $\left.d_{2}, 213 \mathrm{~K}\right): \delta-125.4(\mathrm{~m}, \mathrm{o}),-131.7(\mathrm{~m}, \mathrm{o}),-158.0\left(\mathrm{t},{ }^{3} \mathrm{JFF}_{\mathrm{FF}}\right.$ $=21.3 \mathrm{~Hz}, p),-163.8\left(\mathrm{~m}, \mathrm{~m}^{\prime}\right),-164.6(\mathrm{~m}, \mathrm{~m})\left(\right.$ each $\left.1 \mathrm{~F}, \mathrm{C}_{6} \mathrm{~F}_{5}\right)\left[\Delta \delta^{19} \mathrm{~F}_{\mathrm{mp}}=5.8,6.6\right],-128.4(0)$, $130.7\left(o^{\prime}\right),-159.4(\mathrm{~m}, p),-165.6\left(\mathrm{~m}, m^{\prime}\right),-165.9(\mathrm{~m}, m)\left(\right.$ each $\left.1 \mathrm{~F}, \mathrm{C}_{6} \mathrm{~F}_{5}\right)\left[\Delta \delta^{19} \mathrm{~F}_{\mathrm{mp}}=6.2,6.5\right]$.
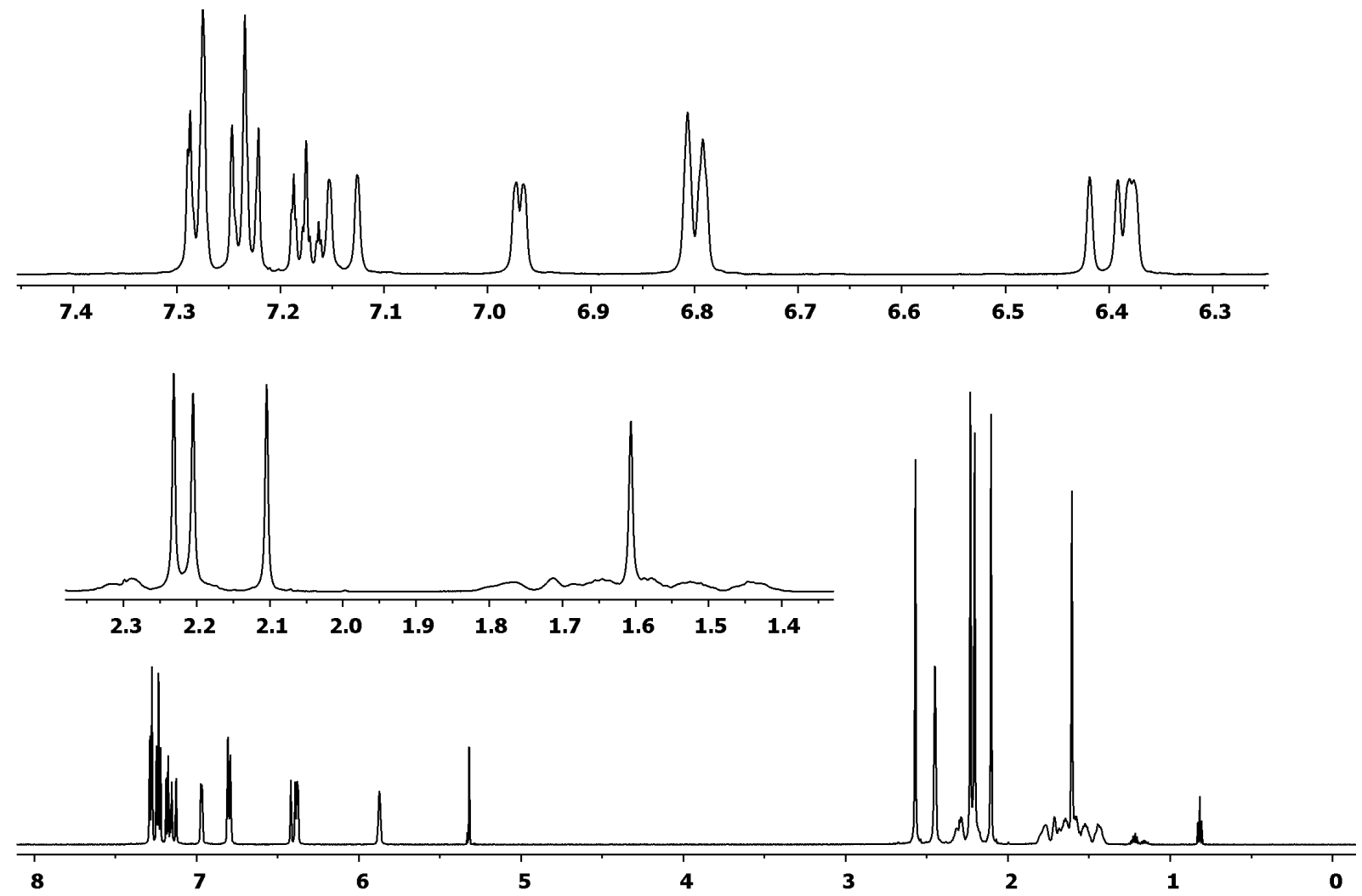

Figure S4: ${ }^{1} \mathbf{H}$ NMR (600 MHz, dichloromethane- $\left.d_{2}, 213 \mathrm{~K}\right)$ spectrum of compound $10 \mathrm{a}$ 

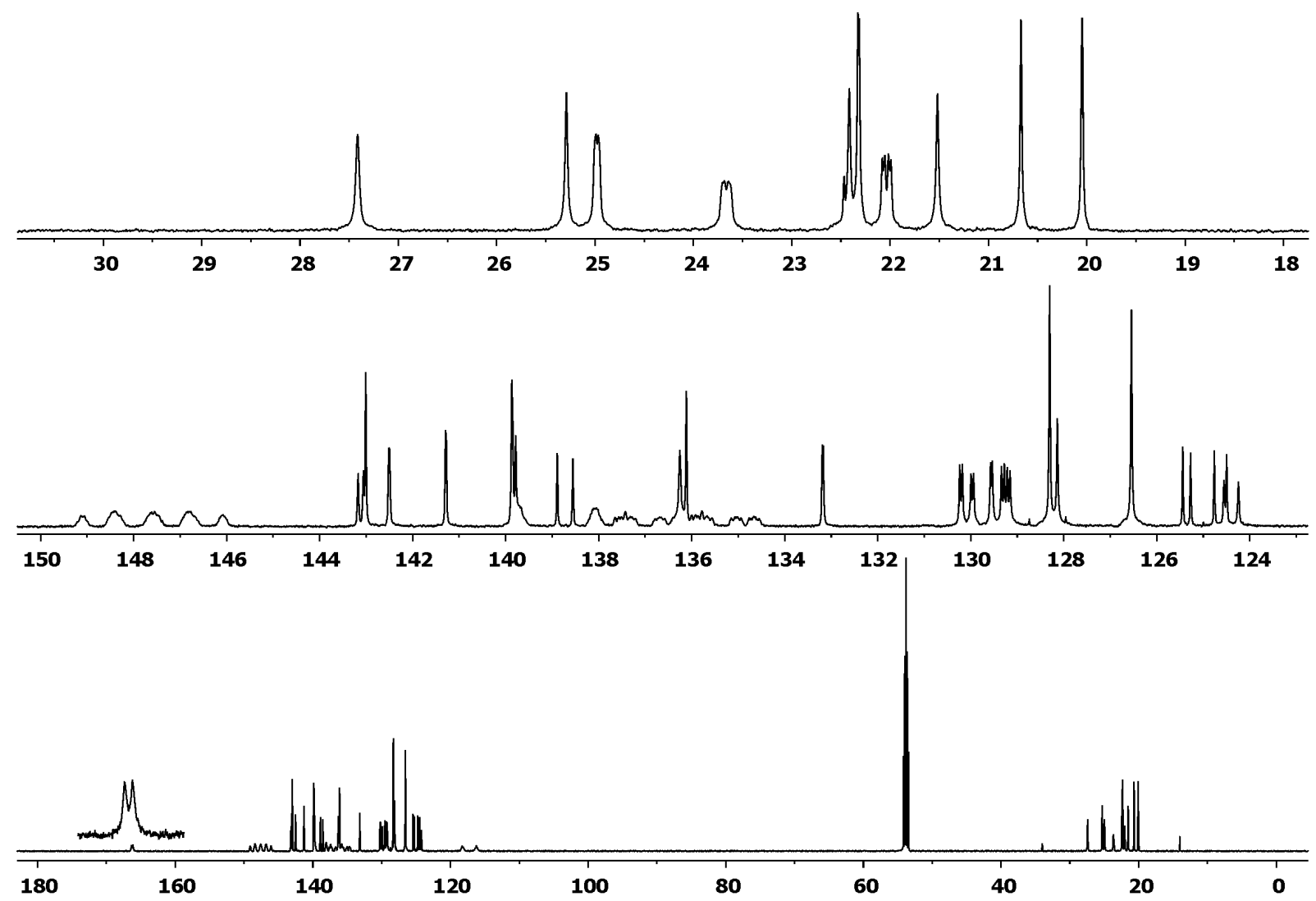

Figure S5: ${ }^{13} \mathrm{C}\left\{{ }^{1} \mathrm{H}\right\}$ NMR $\left(151 \mathrm{MHz}\right.$, dichloromethane- $\left.\mathrm{d}_{2}, 213 \mathrm{~K}\right)$ spectrum of compound 10a

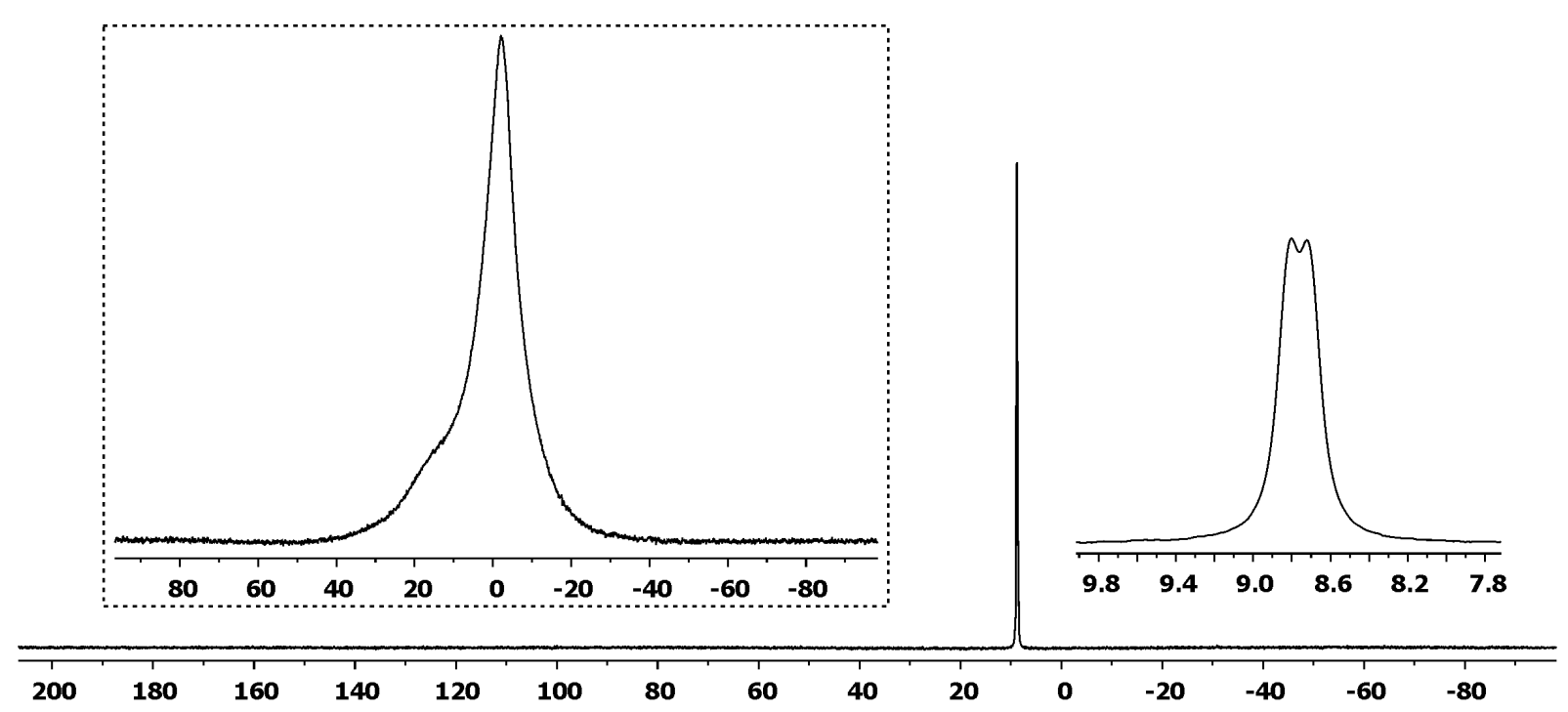

Figure S6: ${ }^{11} \mathbf{B}\left\{{ }^{1} \mathbf{H}\right\}$ NMR $\left(192 \mathrm{MHz}\right.$, dichloromethane- $\left.d_{2}, 213 \mathrm{~K}\right)$ and ${ }^{31} \mathbf{P}\left\{{ }^{1} \mathrm{H}\right\} \mathbf{N M R}(243 \mathrm{MHz}$, dichloromethane$d_{2}, 213 \mathrm{~K}$ ) spectra of compound $10 \mathrm{a}$ 


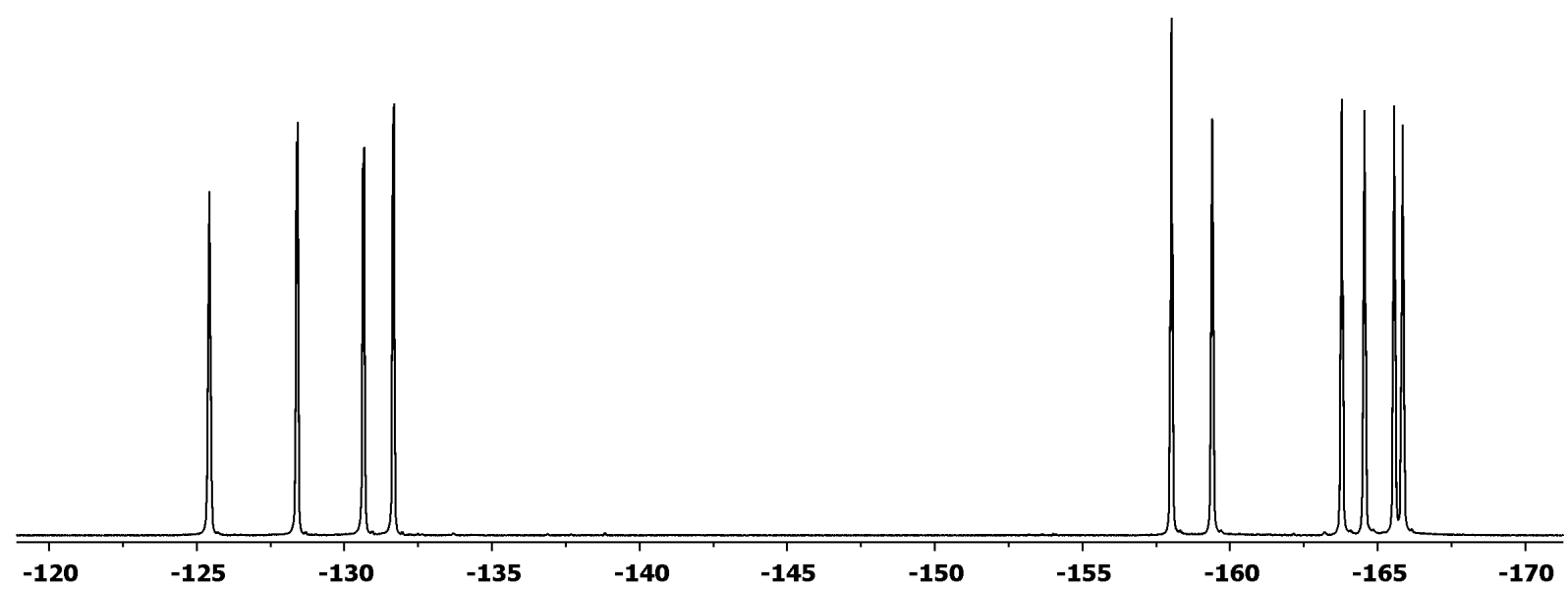

Figure S7: ${ }^{19} \mathrm{~F}$ NMR $\left(564 \mathrm{MHz}\right.$, dichloromethane- $\left.d_{2}, 213 \mathrm{~K}\right)$ spectrum of compound 10a
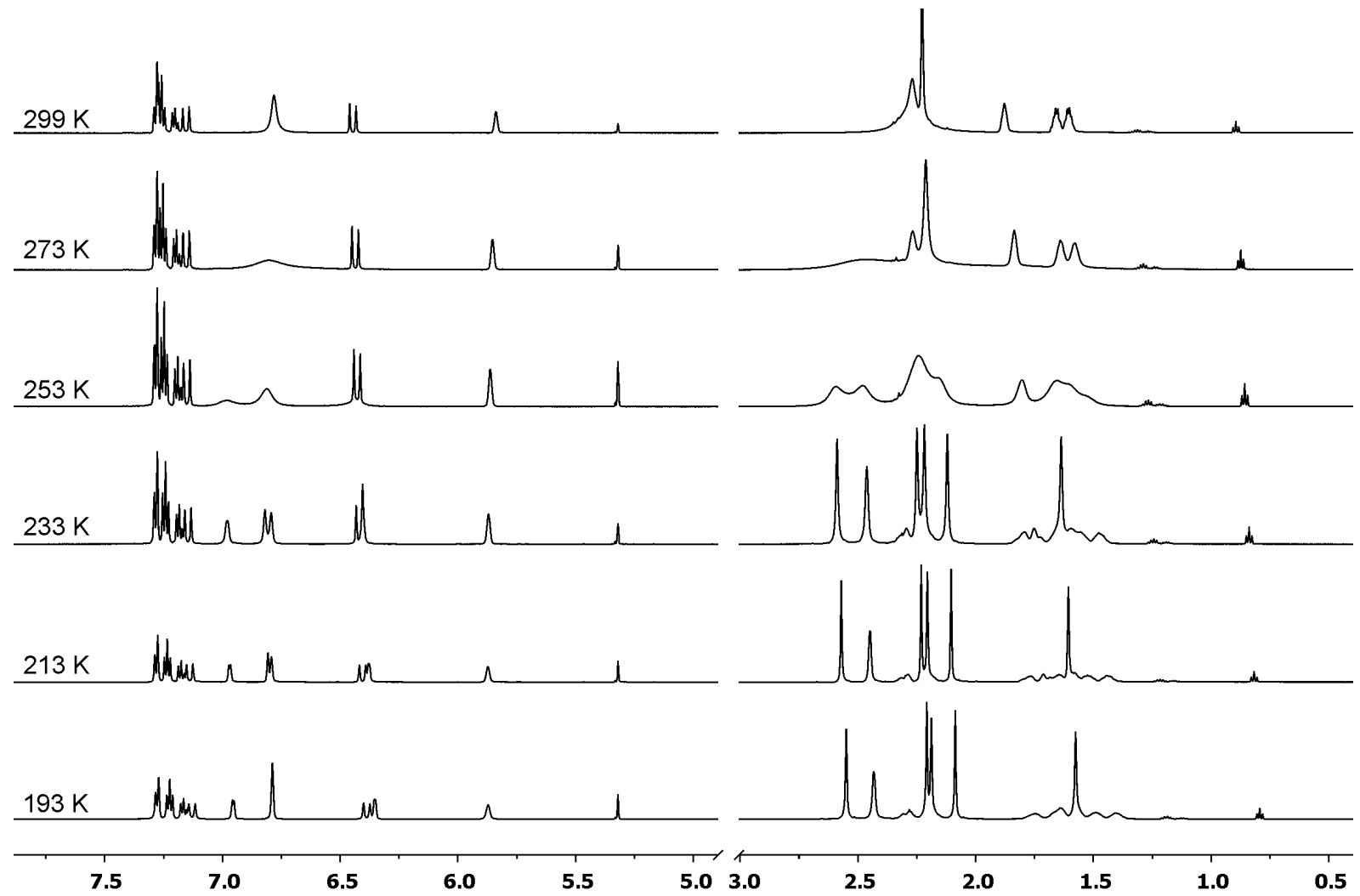

Figure S8: Dynamic ${ }^{1} \mathbf{H}$ NMR $\left(600 \mathrm{MHz}\right.$, dichloromethane- $\left.d_{2}\right)$ spectra of compound 10a 

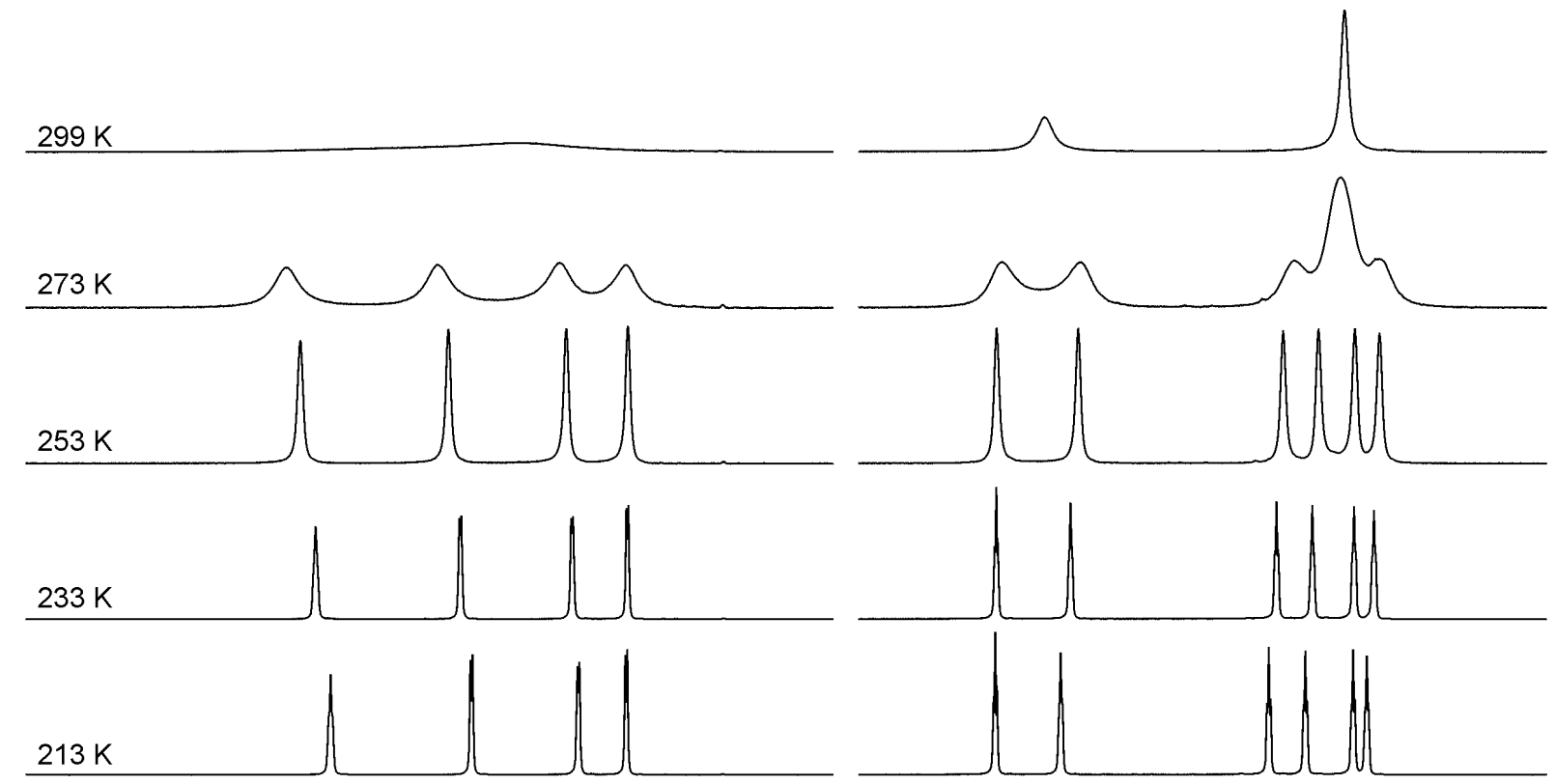

$213 K$
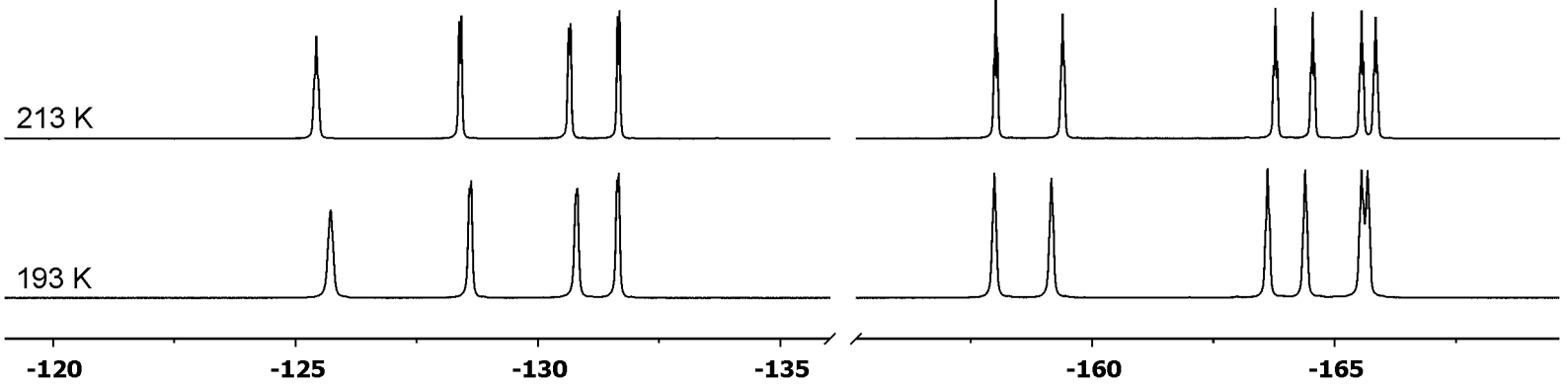

Figure S9: Dynamic ${ }^{19} \mathrm{~F}$ NMR $\left(564 \mathrm{MHz}\right.$, dichloromethane- $\left.d_{2}\right)$ spectra of compound 10a

$\Delta \mathrm{G}^{\ddagger}\left[\mathrm{T}_{\mathrm{c}}, \Delta v(\mathrm{~T})\right]=\mathrm{RT}_{\mathrm{c}}(22.96+\ln (\mathrm{Tc} / \Delta \mathrm{v}))[\mathrm{J} / \mathrm{mol}]$

$\mathrm{T}_{\mathrm{c}}=$ coalescence temperature $[\mathrm{K}]: 286 \mathrm{~K}\left({ }^{19} \mathrm{~F}, p-\mathrm{BC}_{6} \mathrm{~F}_{5}\right)$

$\Delta v=$ chemical shift difference $[\mathrm{Hz}]\left({ }^{19} \mathrm{~F}, p-\mathrm{BC}_{6} \mathrm{~F}_{5}, 233 \mathrm{~K}\right): 883 \mathrm{~Hz}$

$\mathrm{R}=8.314 \mathrm{~J} /(\mathrm{mol} \cdot \mathrm{K}) ; 1 \mathrm{~J}=0.239 \mathrm{cal}$

$\Delta \mathrm{G} \ddagger[286 \mathrm{~K}, \Delta v(233 \mathrm{~K})=883 \mathrm{~Hz}]=51913 \mathrm{~J} / \mathrm{mol}=12.4 \pm 0.3 \mathrm{kcal} / \mathrm{mol}$

Crystals suitable for the X-ray crystal structure analysis were obtained from a dichloromethane solution of compound $10 \mathrm{a}$ at $-35^{\circ} \mathrm{C}$ :

X-ray crystal structure analysis of compound 10a: formula $\mathrm{C}_{46} \mathrm{H}_{38} \mathrm{BF}_{10} \mathrm{P}, M=822.54$, colourless crystal, $0.15 \times 0.10 \times 0.06 \mathrm{~mm}, a=12.4191(2), b=13.0158(2), c=15.8780(3) \AA$, $\alpha$ $=106.250(1), \beta=105.633(1), y=92.883(1)^{\circ}, V=2350.9(1) \AA^{3}, \rho_{\text {calc }}=1.162 \mathrm{gcm}^{-3}, \mu=0.126$ $\mathrm{mm}^{-1}$, empirical absorption correction $(0.981 \leq \mathrm{T} \leq 0.992), Z=2$, triclinic, space group $P \overline{1}$ (No. 2), $\lambda=0.71073 \AA, T=223(2) \mathrm{K}, \omega$ and $\varphi$ scans, 20532 reflections collected $( \pm h, \pm k, \pm l), 7974$ independent $\left(R_{\text {int }}=0.047\right)$ and 6146 observed reflections $[>2 \sigma(I)], 529$ refined parameters, $R$ $=0.070, w R^{2}=0.191$, max. (min.) residual electron density $0.31(-0.31)$ e. $\AA^{-3}$, hydrogen atoms were calculated and refined as riding atoms. 


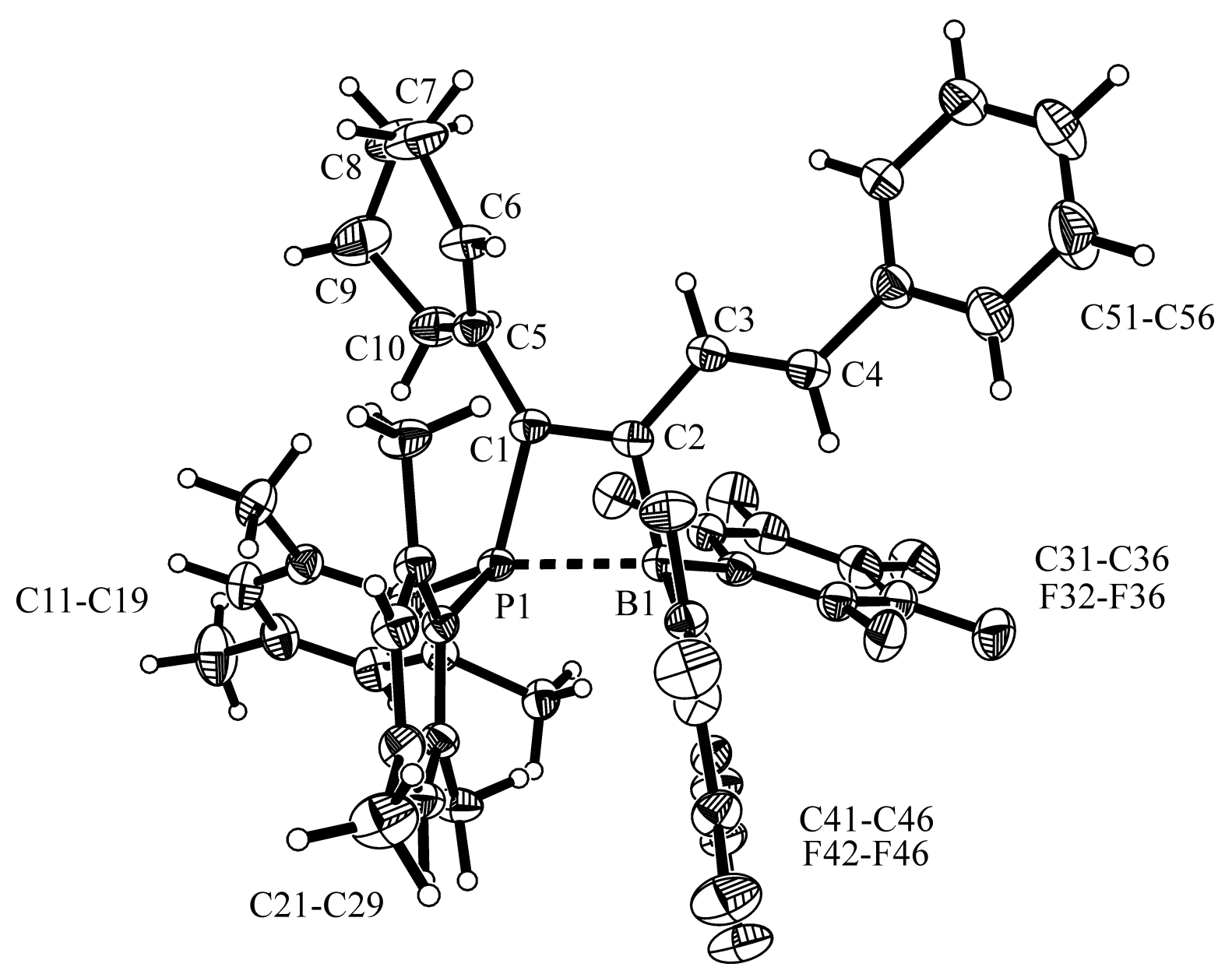

Figure S10: X-ray crystal structure of compound 10a (thermal ellipsoids are shown at the $30 \%$ probability level)

\section{Synthesis of compound $10 b$}

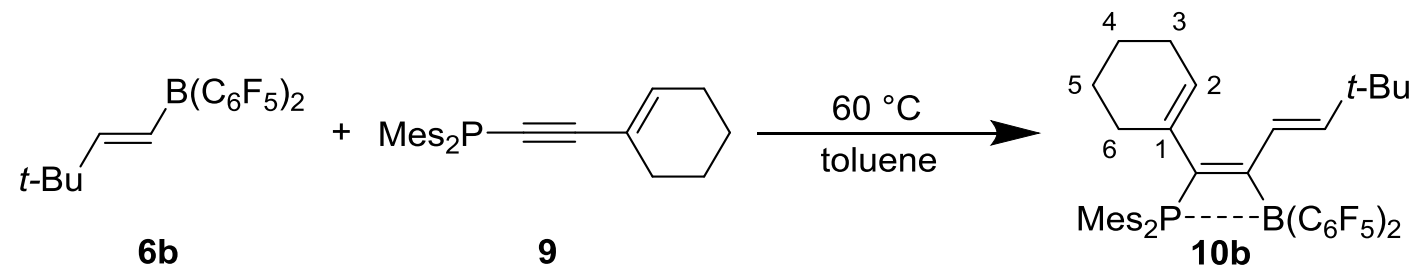

Scheme S3

A solution of borane $6 \mathrm{~b}(1.54 \mathrm{~g}, 3.60 \mathrm{mmol}, 1.0 \mathrm{eq})$ in toluene $(5 \mathrm{~mL})$ was added dropwise to a solution of phosphane $9(1.35 \mathrm{~g}, 3.60 \mathrm{mmol}, 1.0 \mathrm{eq})$ in toluene $(5 \mathrm{~mL})$. The reaction mixture was stirred at $60^{\circ} \mathrm{C}$ for 3 days and then all volatiles were removed in vacuo. The resulting sticky red residue was dissolved in pentane $(5 \mathrm{~mL})$, which was subsequently removed in vacuo. The obtained solid was washed with pentane $(5 \times 3 \mathrm{~mL})$ and dried to finally give compound $10 \mathrm{~b}$ as an off-white solid (1.26 g, $1.6 \mathrm{mmol}, 43 \%)$.

IR $(\mathrm{KBr}): \tilde{v}\left[\mathrm{~cm}^{-1}\right]=5340(\mathrm{w}), 4382(\mathrm{w}), 4310(\mathrm{w}), 3901(\mathrm{w}), 3819(\mathrm{w}), 3747(\mathrm{w}), 3673(\mathrm{w})$, $3628(w), 3156(w), 3026(s), 2954(s), 2863(s), 2739(w), 2632(w), 2568(w), 2400(w)$, 
$2360(w), 2220(w), 2093(w), 1828(w), 1772(w), 1735(w), 1699(w), 1645(s), 1605(m)$, $1515(\mathrm{~m}), 1445(\mathrm{~m}), 1381(\mathrm{~m}), 1177(\mathrm{w}), 1114(\mathrm{~s}), 1032(\mathrm{~m}), 966(\mathrm{~m}), 925(\mathrm{~m}), 854(\mathrm{~s})$, $805(\mathrm{~m}), 772(\mathrm{~m}), 743(\mathrm{~s}), 695(\mathrm{~s}), 668(\mathrm{~s}), 630(\mathrm{~s}), 609(\mathrm{~s}), 574(\mathrm{~m}), 554(\mathrm{~m}), 533(\mathrm{~m})$, $498(\mathrm{~m}), 456(\mathrm{~m}), 417(\mathrm{~m})$.

Decomp. $212^{\circ} \mathrm{C}$.

Anal. Calc. for $\mathrm{C}_{44} \mathrm{H}_{42} \mathrm{BF}_{10} \mathrm{P}: \mathrm{C}: 65.85 ; \mathrm{H}: 5.27$. Found: C: $65.79 ; \mathrm{H}: 5.21$.

${ }^{1} \mathrm{H}$ NMR $\left(500 \mathrm{MHz}\right.$, dichloromethane- $\left.d_{2}, 213 \mathrm{~K}\right): \delta 6.95\left(\mathrm{~d},{ }^{4} \mathrm{JPH}_{\mathrm{PH}}=3.0 \mathrm{~Hz}, 1 \mathrm{H}, m-\mathrm{Mes}^{\mathrm{a}}\right), 6.78(\mathrm{~s}$, $\left.1 \mathrm{H}, m^{\prime}-\mathrm{Mes}^{\mathrm{a}}\right), 6.76\left(\mathrm{~s}, 1 \mathrm{H}, m^{\prime}-\mathrm{Mes}^{\mathrm{b}}\right), 6.34\left(\mathrm{~s}, 1 \mathrm{H}, m-\mathrm{Mes}^{\mathrm{b}}\right), 6.15\left(\mathrm{~d},{ }^{3} \mathrm{JHH}_{\mathrm{H}}=16.0 \mathrm{~Hz}, 1 \mathrm{H},=\mathrm{CH}\right)$, $5.72(\mathrm{~s}, 1 \mathrm{H}, 2-\mathrm{CH}), 5.62\left(\mathrm{~d},{ }^{3} \mathrm{JHH}_{\mathrm{HH}}=16.0 \mathrm{~Hz}, 1 \mathrm{H},=\mathrm{CH}^{\mathrm{tBu}}\right), 2.53\left(\mathrm{~s}, 3 \mathrm{H}, \mathrm{o}^{\prime}-\mathrm{CH}_{3}{ }^{\mathrm{Mes}, \mathrm{b}}\right), 2.44(\mathrm{br}, 3 \mathrm{H}$, $o-\mathrm{CH}_{3}{ }^{\mathrm{Mes}, \mathrm{a}}$ ), 2.21 (s, 3H, $p-\mathrm{CH}_{3}{ }^{\mathrm{Mes}, \mathrm{a}}$ ), 2.18, 2.13 (each m, each $\left.1 \mathrm{H}, 3-\mathrm{CH}_{2}\right)^{\mathrm{t}}, 2.16\left(\mathrm{~s}, 3 \mathrm{H}, \mathrm{o}^{\prime}-\right.$ $\mathrm{CH}_{3}{ }^{\mathrm{Mes}, \mathrm{a}}$ ), 2.09 (s, 3H, $p-\mathrm{CH}_{3}{ }^{\mathrm{Mes}, \mathrm{b}}$ ), 1.71, 1.67 (each br m, each $\left.1 \mathrm{H}, 5-\mathrm{CH}_{2}\right)^{\mathrm{t}}, 1.59,1.45$ (each $\mathrm{m}$, each $\left.1 \mathrm{H}, 4-\mathrm{CH}_{2}\right), 1.56\left(\mathrm{~s}, 3 \mathrm{H}, \mathrm{o}-\mathrm{CH}_{3}{ }^{\mathrm{Mes}, \mathrm{b}}\right), 1.53,1.40$ (each m, each $\left.1 \mathrm{H}, 6-\mathrm{CH}_{2}\right), 0.75(\mathrm{~s}$, $\left.9 \mathrm{H}, \mathrm{CH}_{3}{ }^{\mathrm{tBu}}\right)$. [ ${ }^{\mathrm{t}}$ tentatively assigned]

${ }^{13} \mathrm{C}\left\{{ }^{1} \mathrm{H}\right\}$ NMR (126 MHz, dichloromethane- $\left.d_{2}, 213 \mathrm{~K}\right): \delta 167.9\left(\mathrm{br} \mathrm{d},{ }^{2} \mathrm{JPC}_{\mathrm{PC}}=30 \mathrm{~Hz}, \mathrm{BC}=\right), 151.9$ $\left(=\mathrm{CH}^{\mathrm{tBu}}\right), 143.1\left(\mathrm{~d},{ }^{2} \mathrm{JPC}_{\mathrm{PC}}=17.2 \mathrm{~Hz}, \mathrm{o}-\mathrm{Mes}^{\mathrm{a}}\right), 143.0\left(o^{\prime}-\mathrm{Mes}^{\mathrm{a}}\right), 142.5\left(\mathrm{~d},{ }^{2} \mathrm{JPC}_{\mathrm{PC}}=3.4 \mathrm{~Hz}, \mathrm{o}^{\prime}-\mathrm{Mes}^{\mathrm{b}}\right)$, $141.1\left(\mathrm{~d},{ }^{4} \mathrm{~J}_{\mathrm{PC}}=1.7 \mathrm{~Hz}, p-\mathrm{Mes}^{\mathrm{a}}\right), 139.7\left(\mathrm{~d},{ }^{2} \mathrm{~J}_{\mathrm{PC}}=13.2 \mathrm{~Hz}, o-\mathrm{Mes}^{\mathrm{b}}\right), 139.7\left(\mathrm{~d},{ }^{4} \mathrm{JPC}_{\mathrm{PC}}=2.1 \mathrm{~Hz}, p-\right.$ $\left.M{ }^{b}\right), 135.5\left(d,{ }^{1} J_{P C}=51.1 \mathrm{~Hz}, P C=\right), 133.0\left(d,{ }^{2} J_{P C}=3.5 \mathrm{~Hz}, 1-C=\right), 130.1\left(d,{ }^{3} J_{P C}=8.2 \mathrm{~Hz}\right.$, $\left.m^{\prime}-\mathrm{Mes}^{\mathrm{b}}\right), 129.5\left(\mathrm{~d},{ }^{3} J_{\mathrm{PC}}=6.9 \mathrm{~Hz}, 2-\mathrm{CH}\right), 129.5\left(\mathrm{~d},{ }^{3} J_{\mathrm{PC}}=6.9 \mathrm{~Hz}, m^{\prime}-\mathrm{Mes}^{\mathrm{a}}\right), 129.3\left(\mathrm{~d},{ }^{3} J_{\mathrm{PC}}=\right.$ $\left.9.1 \mathrm{~Hz}, m-\mathrm{Mes}^{\mathrm{a}}\right), 129.1$ (d, $\left.{ }^{3} \mathrm{JPC}_{\mathrm{PC}}=9.1 \mathrm{~Hz}, m-\mathrm{Mes}^{\mathrm{b}}\right), 125.6\left(\mathrm{~d},{ }^{1} \mathrm{~J}_{\mathrm{PC}}=25.0 \mathrm{~Hz}, i-\mathrm{Mes}^{\mathrm{a}}\right), 124.8(\mathrm{~d}$, $\left.{ }^{1} J_{\mathrm{PC}}=39.6 \mathrm{~Hz}, i-\mathrm{Mes}^{\mathrm{b}}\right), 120.2\left(\mathrm{~d},{ }^{3} J_{\mathrm{PC}}=45.5 \mathrm{~Hz},=\mathrm{CH}\right), 33.3\left(\mathrm{C}^{\mathrm{tBu}}\right), 28.0\left(\mathrm{CH}_{3}^{\mathrm{tBu}}\right), 27.4\left(5-\mathrm{CH}_{2}\right)^{\mathrm{t}}$, $25.2\left(3-\mathrm{CH}_{2}\right)^{\mathrm{t}}, 24.9\left(\mathrm{~m}, \mathrm{o}^{\prime}-\mathrm{CH}_{3}{ }^{\mathrm{Mes}, \mathrm{b}}\right), 23.6\left(\mathrm{~m}, \mathrm{o}-\mathrm{CH}_{3}{ }^{\mathrm{Mes}, \mathrm{a}}\right), 22.4\left(6-\mathrm{CH}_{2}\right)^{\mathrm{t}}, 22.3\left(\mathrm{~d},{ }^{3} \mathrm{JPC}_{\mathrm{PC}}=2.3 \mathrm{~Hz}\right.$, $\left.o^{\prime}-\mathrm{CH}_{3}{ }^{\text {Mes,a }}\right), 22.0\left(\mathrm{dd}, J=9.3 \mathrm{~Hz}, J=3.4 \mathrm{~Hz}, o-\mathrm{CH}_{3}{ }^{\text {Mes,b}}\right), 21.5\left(4-\mathrm{CH}_{2}\right)^{\mathrm{t}}, 20.7\left(p-\mathrm{CH}_{3}{ }^{\text {Mes,a }}\right), 20.0$ $\left(p-\mathrm{CH}_{3}{ }^{\mathrm{Mes}, \mathrm{b}}\right) \cdot\left[\mathrm{C}_{6} \mathrm{~F}_{5}\right.$ not listed; ${ }^{t}$ tentatively assigned]

${ }^{31} \mathbf{P}\left\{{ }^{1} \mathrm{H}\right\}$ NMR $\left(202 \mathrm{MHz}\right.$, dichloromethane- $\left.d_{2}, 213 \mathrm{~K}\right): \delta 10.9$ (partial relaxed 1:1:1:1 q JPB $26 \mathrm{~Hz})$.

${ }^{31} \mathbf{P}\left\{{ }^{1} \mathrm{H}\right\}$ NMR $\left(202 \mathrm{MHz}\right.$, dichloromethane- $\left.d_{2}, 299 \mathrm{~K}\right): \delta 12.8\left(v_{1 / 2} \sim 37 \mathrm{~Hz}\right)$.

${ }^{11} \mathrm{~B}\left\{{ }^{1} \mathrm{H}\right\}$ NMR $\left(160 \mathrm{MHz}\right.$, dichloromethane- $\left.d_{2}, 213 \mathrm{~K}\right): \delta-3.0\left(\mathrm{v}_{1 / 2} \sim 2400 \mathrm{~Hz}\right)$.

${ }^{19} \mathrm{~F}$ NMR $\left(470 \mathrm{MHz}\right.$, dichloromethane- $\left.d_{2}, 213 \mathrm{~K}\right): \delta-125.9(\mathrm{~m}, \mathrm{o}),-131.2(\mathrm{~m}, 0),-158.7(\mathrm{br} \mathrm{t}$, $\left.{ }^{3} J_{\mathrm{FF}}=21.3 \mathrm{~Hz}, p\right), 164.7(\mathrm{~m}, \mathrm{~m}),-164.9\left(\mathrm{~m}, \mathrm{~m}^{\prime}\right)\left(\right.$ each $\left.1 \mathrm{~F}, \mathrm{C}_{6} \mathrm{~F}_{5}\right)\left[\Delta \delta^{19} \mathrm{~F}_{\mathrm{m}, \mathrm{p}}=6.0,6.2\right] ;-128.8(\mathrm{~m}$, o), -130.7 (m, o), -159.8 (br t, $\left.{ }^{3} J_{\mathrm{FF}}=17.0 \mathrm{~Hz}, p\right),-165.9(\mathrm{~m}, m),-166.1(\mathrm{~m}, m)($ each $1 \mathrm{~F}$, $\left.\mathrm{C}_{6} \mathrm{~F}_{5}\right)\left[\Delta \delta^{19} \mathrm{~F}_{\mathrm{m}, \mathrm{p}}=6.1,6.3\right]$ 


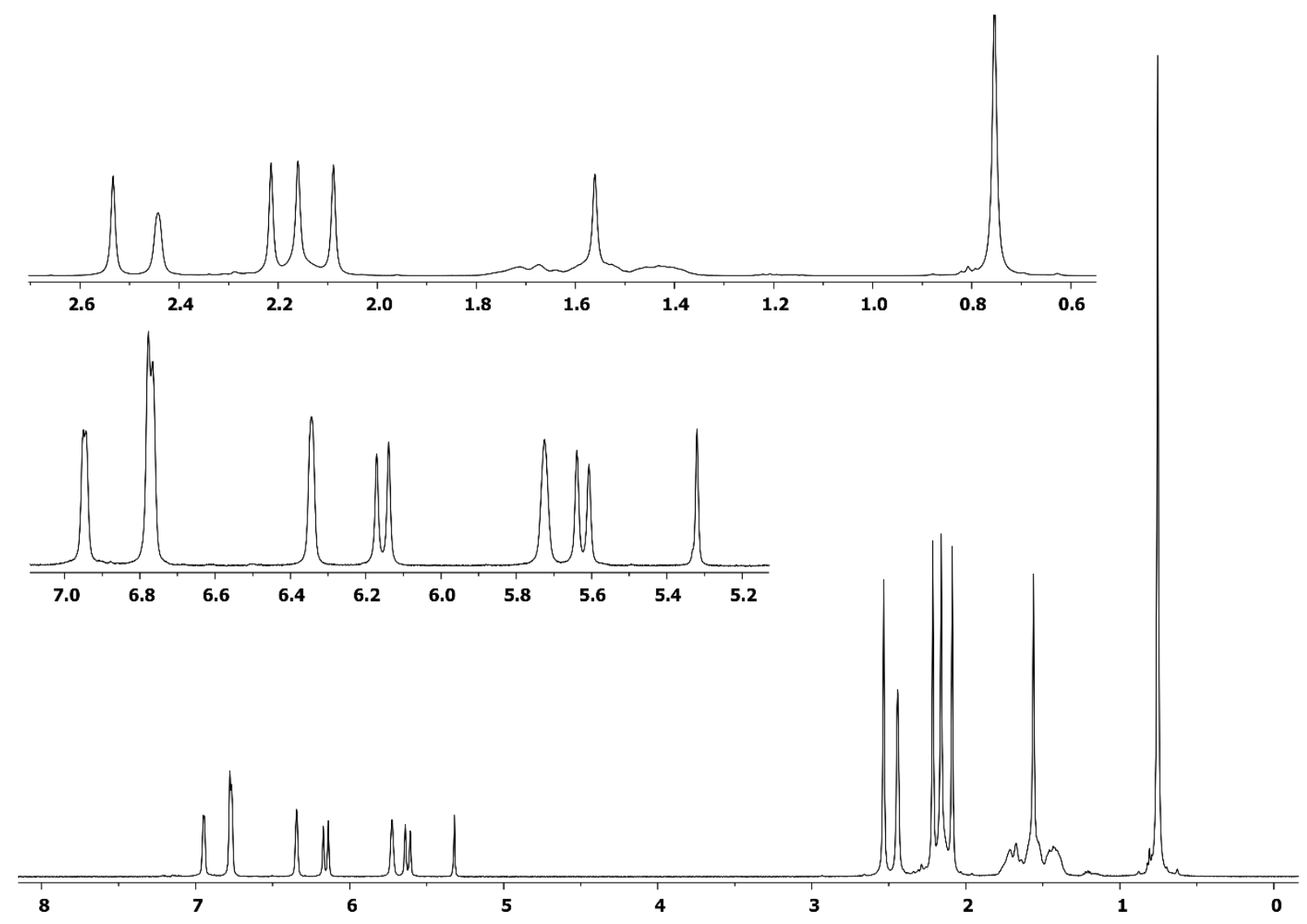

Figure S11: ${ }^{1} \mathbf{H}$ NMR $\left(500 \mathrm{MHz}\right.$, dichloromethane- $\left.d_{2}, 213 \mathrm{~K}\right)$ spectrum of compound 10b
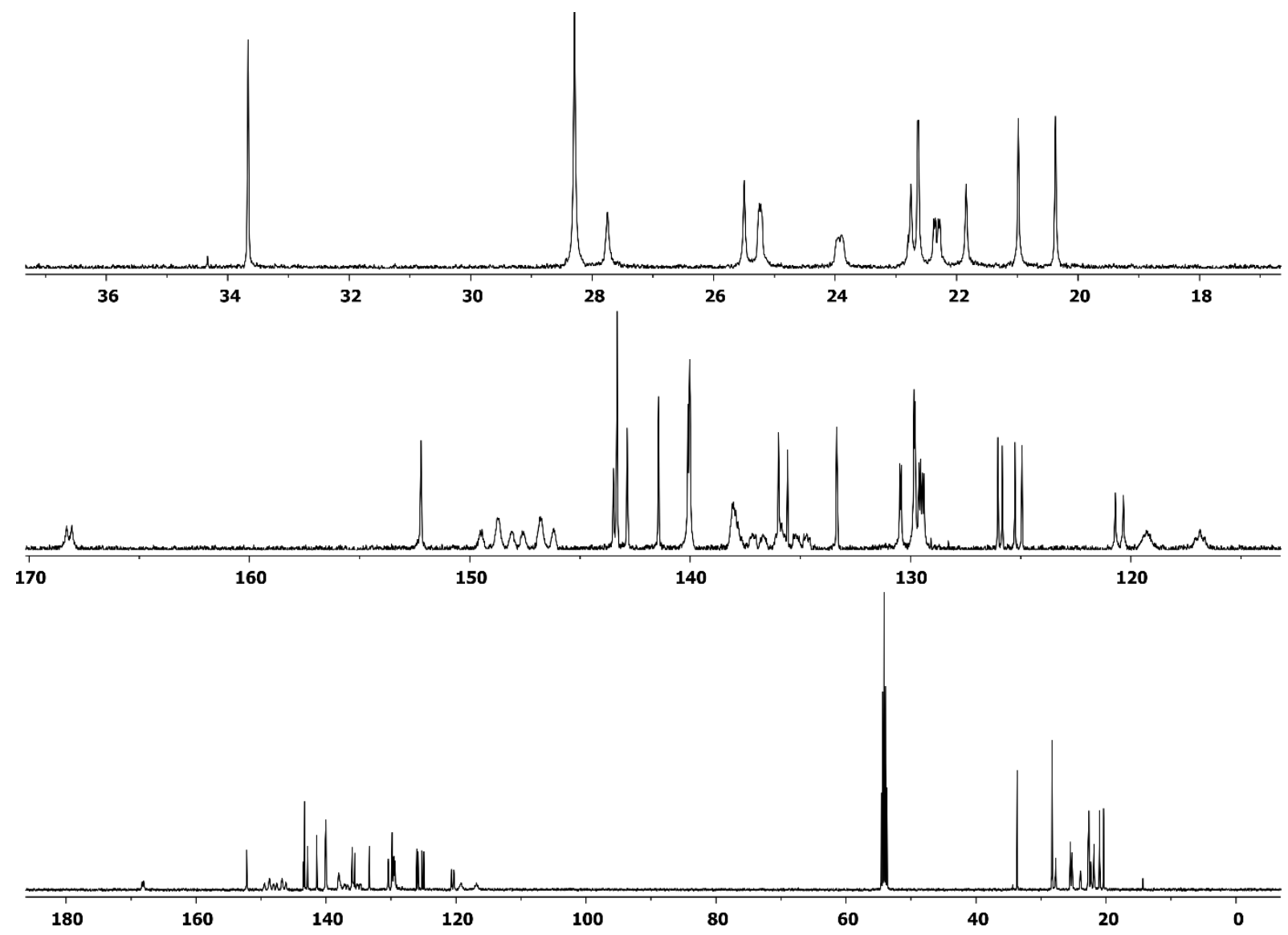

Figure S12: ${ }^{13} \mathrm{C}\left\{{ }^{1} \mathrm{H}\right\}$ NMR (126 MHz, dichloromethane- $\left.d_{2}, 213 \mathrm{~K}\right)$ spectrum of compound $10 \mathrm{~b}$ 


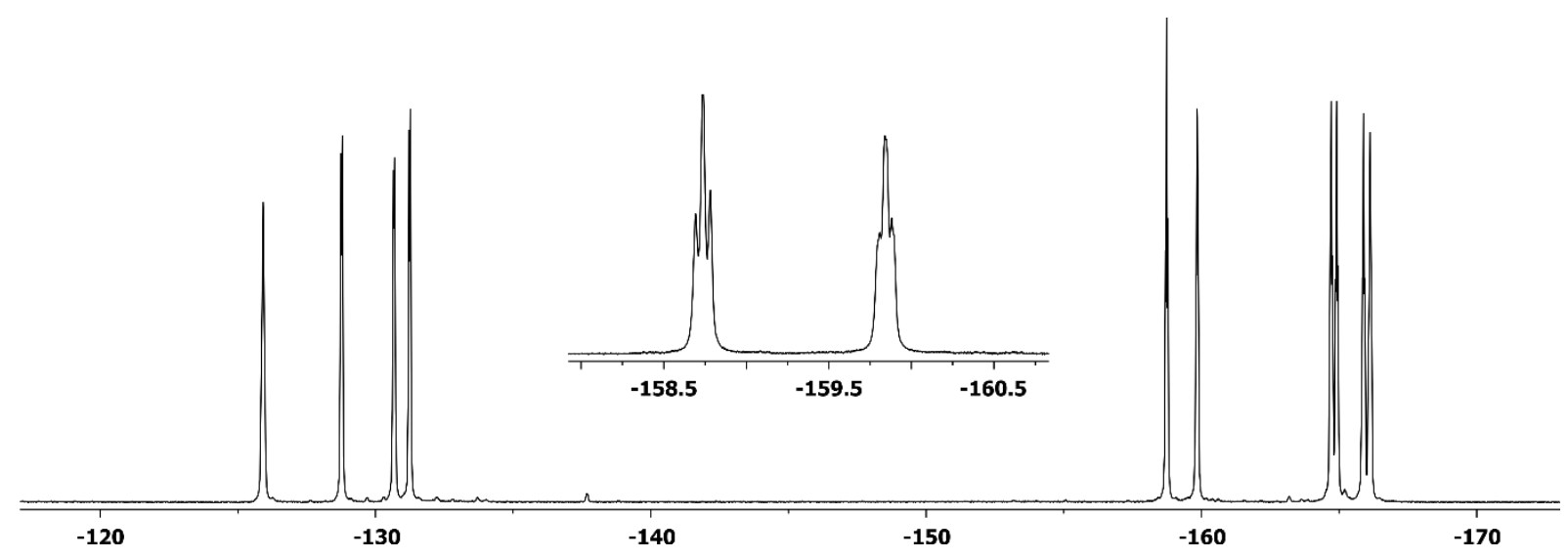

Figure S13: ${ }^{19} \mathrm{~F}$ NMR (470 MHz, dichloromethane- $\left.d_{2}, 213 \mathrm{~K}\right)$ spectrum of compound 10b

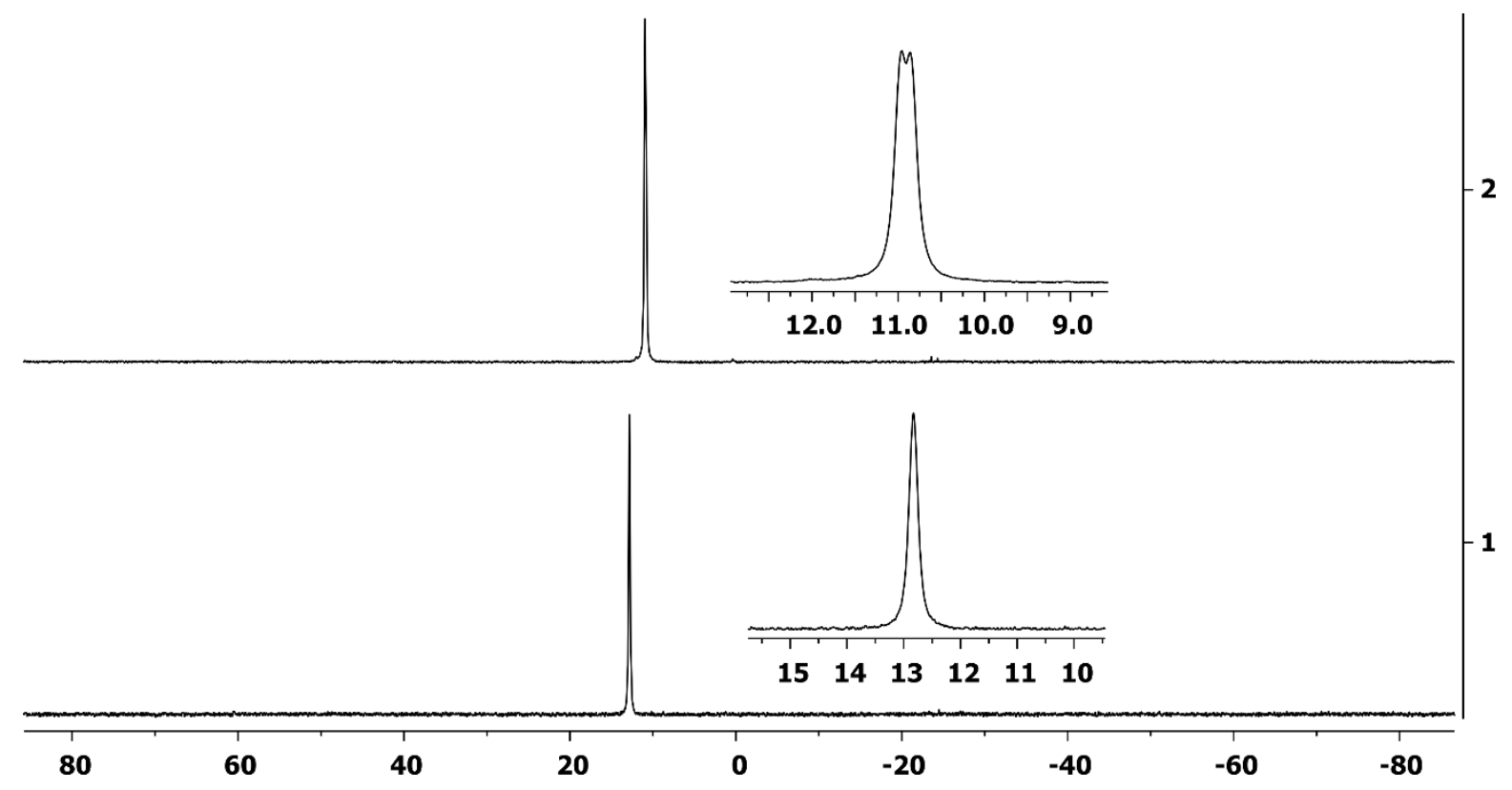

Figure S14: ${ }^{31} \mathrm{P}\left\{{ }^{1} \mathrm{H}\right\}$ NMR (202 MHz, dichloromethane- $\left.d_{2}\right)$ spectra of compound $10 \mathrm{~b}$ at $299 \mathrm{~K}(1)$ and $213 \mathrm{~K}(2)$

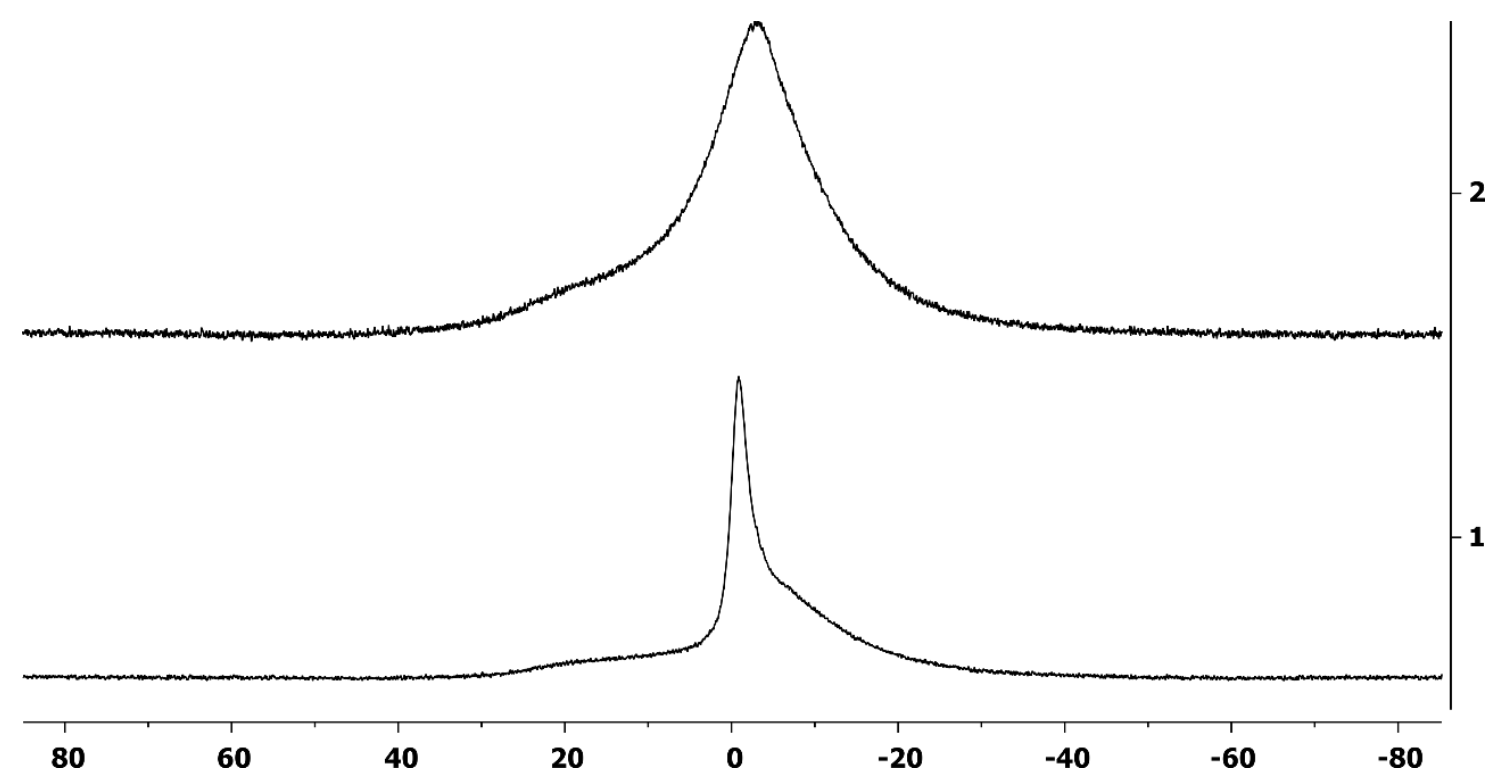

Figure S15: ${ }^{11} \mathrm{~B}\left\{{ }^{1} \mathrm{H}\right\}$ NMR (160 MHz, dichloromethane- $\left.d_{2}\right)$ spectra of compound $10 \mathrm{~b}$ at $299 \mathrm{~K}(1)$ and $213 \mathrm{~K}(2)$ 


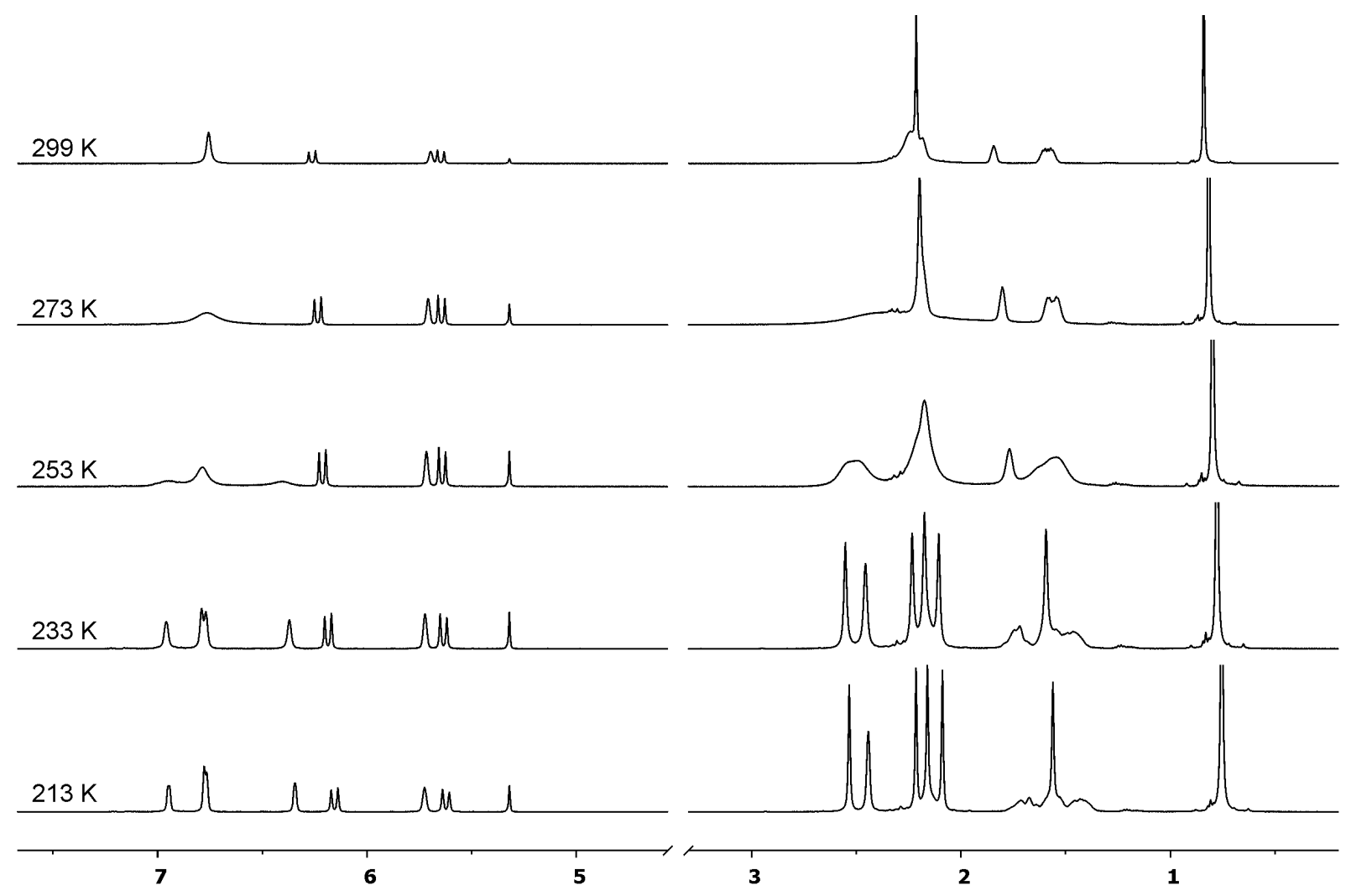

Figure S16: Dynamic ${ }^{1} \mathrm{H}$ NMR (500 MHz, dichloromethane-d2) spectra of compound 10b
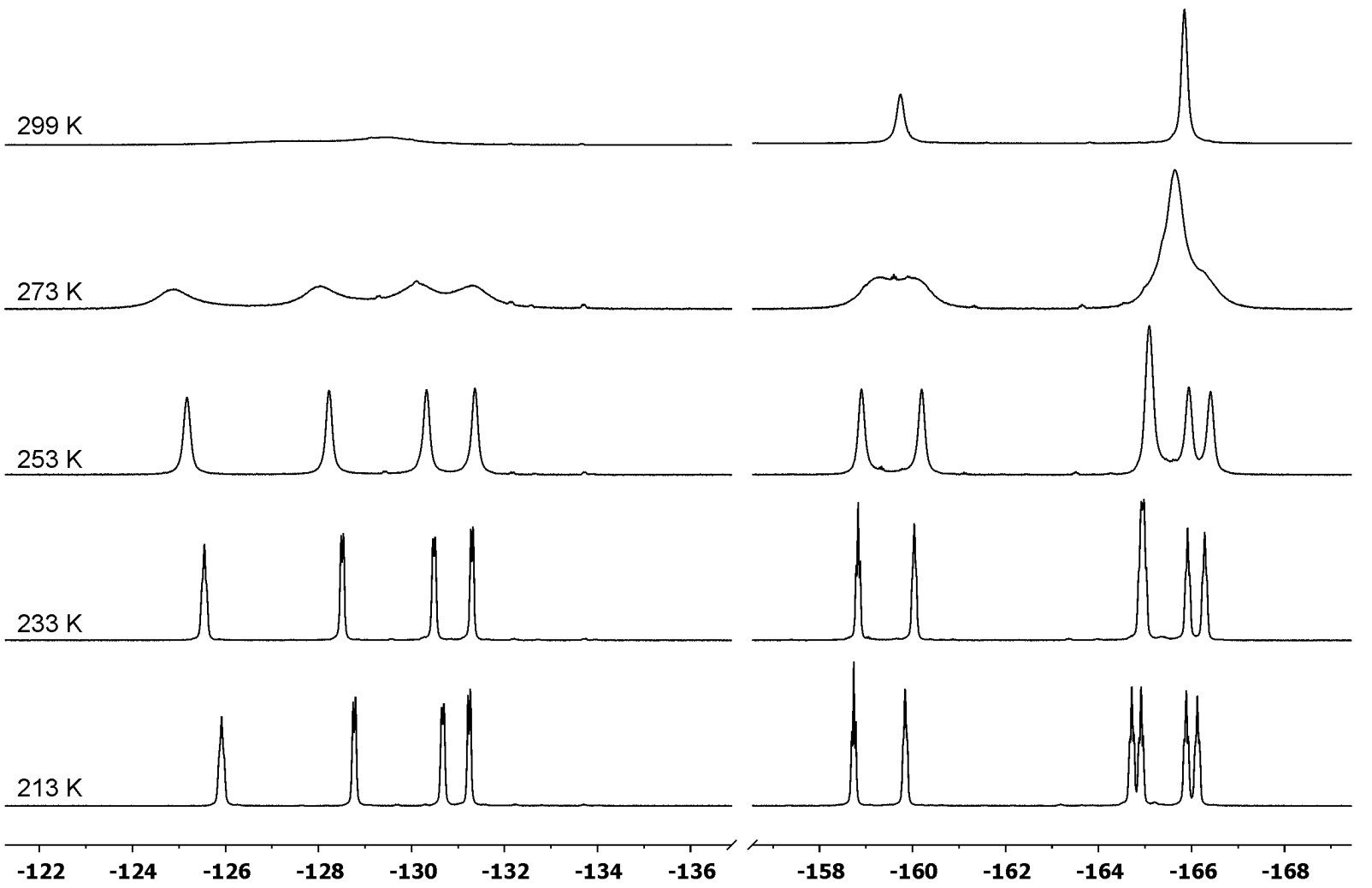

Figure S17: Dynamic ${ }^{19} \mathrm{~F}$ NMR (470 MHz, dichloromethane- $\left.d_{2}\right)$ spectra of compound 10b 
$\Delta \mathrm{G}^{\ddagger}\left[\mathrm{T}_{\mathrm{c}}, \Delta v(\mathrm{~T})\right]=\mathrm{RT}_{\mathrm{c}}(22.96+\ln (\mathrm{Tc} / \Delta v))[\mathrm{J} / \mathrm{mol}]$

$\mathrm{T}_{\mathrm{c}}=$ coalescence temperature $[\mathrm{K}]: 286 \mathrm{~K}\left({ }^{19} \mathrm{~F}, p-\mathrm{BC}_{6} \mathrm{~F}_{5}\right)$

$\Delta v=$ chemical shift difference $[\mathrm{Hz}]\left({ }^{19} \mathrm{~F}, p-\mathrm{BC}_{6} \mathrm{~F}_{5}, 233 \mathrm{~K}\right): 567 \mathrm{~Hz}$

$\mathrm{R}=8.314 \mathrm{~J} /(\mathrm{mol} \cdot \mathrm{K}) ; 1 \mathrm{~J}=0.239 \mathrm{cal}$

$\Delta \mathrm{G} \ddagger[286 \mathrm{~K}, \Delta v(233 \mathrm{~K})=567 \mathrm{~Hz}]=52967 \mathrm{~J} / \mathrm{mol}=12.7 \pm 0.3 \mathrm{kcal} / \mathrm{mol}$

Crystals suitable for the X-ray crystal structure analysis were obtained from a solution of $\mathbf{1 0 b}$ in pentane at $-35^{\circ} \mathrm{C}$ :

X-ray crystal structure analysis of compound $10 \mathrm{~b}$ : formula $\mathrm{C}_{44} \mathrm{H}_{42} \mathrm{BF}{ }_{10} \mathrm{P}, M=802.56$, colourless crystal, $0.22 \times 0.06 \times 0.01 \mathrm{~mm}, a=12.7265(3), b=13.0670(3), c=15.1809(6) \AA, \alpha$ $=103.041(1), \beta=98.995(1), \gamma=102.917(2)^{\circ}, V=2339.7(1) \AA^{3}, \rho_{\text {calc }}=1.139 \mathrm{gcm}^{-3}, \mu=0.125$ $\mathrm{mm}^{-1}$, empirical absorption correction $(0.973 \leq \mathrm{T} \leq 0.998), Z=2$, triclinic, space group $P^{\overline{1}}$ (No. 2), $\lambda=0.71073 \AA, T=223(2) \mathrm{K}, \omega$ and $\varphi$ scans, 21518 reflections collected $( \pm h, \pm k, \pm l), 8122$ independent $\left(R_{\text {int }}=0.067\right)$ and 5633 observed reflections $[1>2 \sigma(I)], 603$ refined parameters, $R$ $=0.087, w R^{2}=0.233$, max. (min.) residual electron density $0.29(-0.29)$ e. $\AA^{-3}$, hydrogen atoms were calculated and refined as riding atoms.

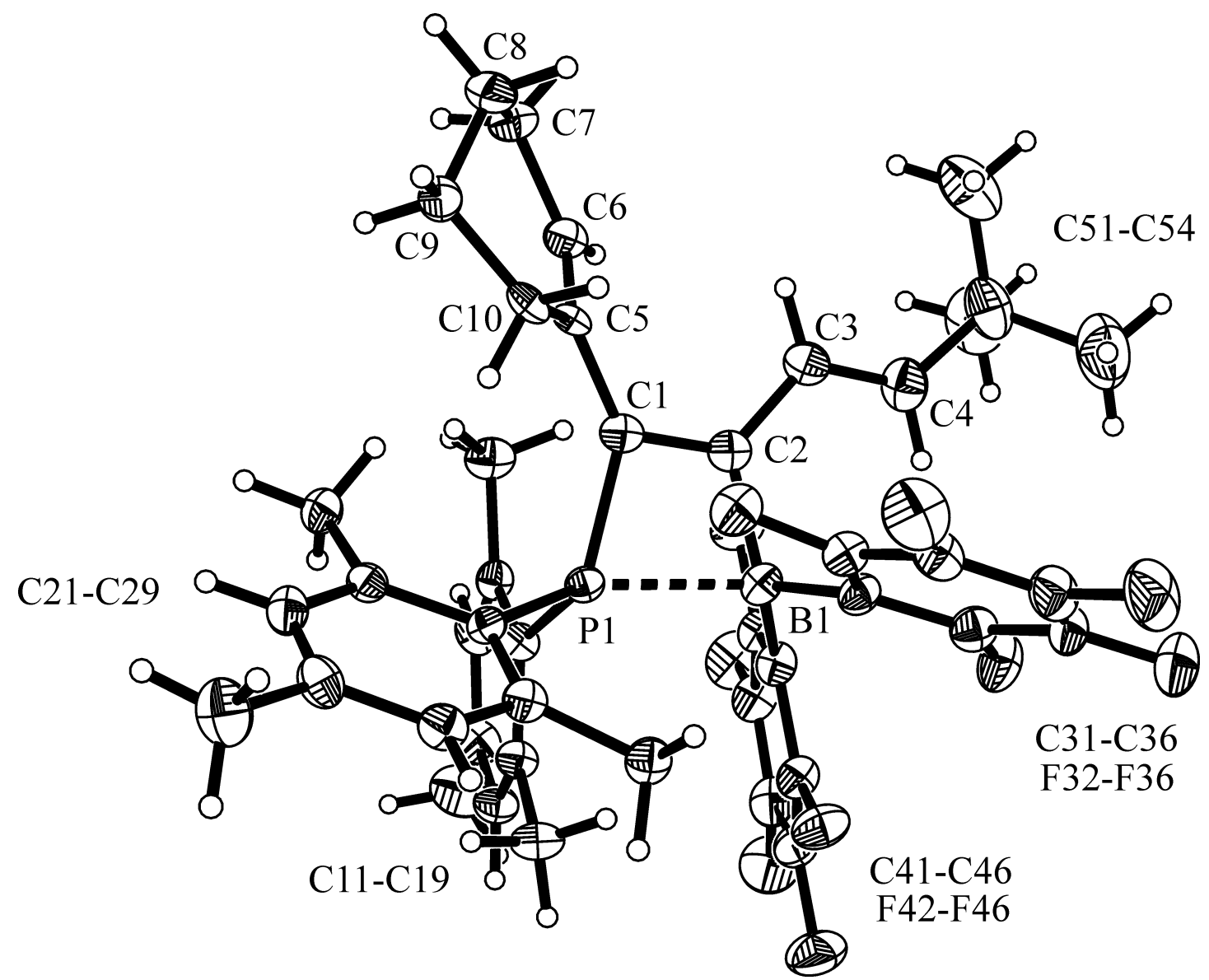

Figure S18: X-ray crystal structure of compound 10b (thermal ellipsoids are shown at the $30 \%$ probability level) 


\section{Synthesis of compound 11}

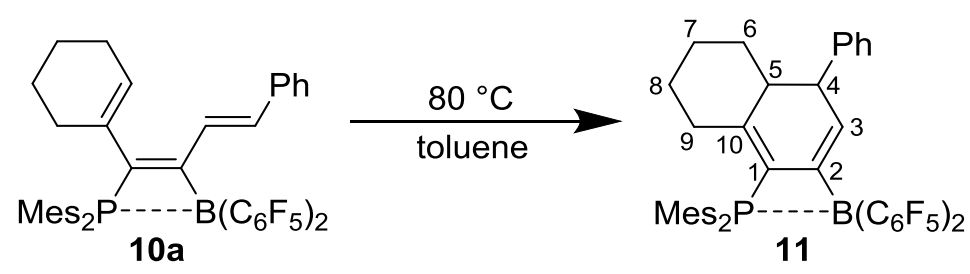

Scheme S4

A solution of compound 10a $(515.2 \mathrm{mg}, 626.3 \mu \mathrm{mol}, 1.0 \mathrm{eq})$ in toluene $(5 \mathrm{~mL})$ was stirred at $80^{\circ} \mathrm{C}$ for 3 days. Then all volatiles were removed in vacuo and the resulting sticky residue was dissolved in pentane $(3 \mathrm{~mL})$, which was subsequently removed in vacuo. After suspending the obtained sticky solid in pentane $(3 \mathrm{~mL})$ the precipitate was collected, washed with pentane $(3 \times 2 \mathrm{~mL})$ and dried in vacuo to give compound 11 as a white solid (454.2 mg, $552.2 \mu \mathrm{mol}$, $88 \%)$.

IR $(\mathrm{KBr}): \tilde{v}\left[\mathrm{~cm}^{-1}\right]=3696(\mathrm{w}), 3027(\mathrm{~m}), 2959(\mathrm{~m}), 2934(\mathrm{~s}), 2856(\mathrm{~m}), 2797(\mathrm{w}), 2295(\mathrm{w}), 1642$ (m), 1602 (s), 1556 (w), 1515 (s), 1460 (s), $1384(\mathrm{~m}), 1283(\mathrm{~m}), 1246$ (m), 1204 (w), 1094 (s), 1040 (w), 1011 (w), 968 (s), 907 (w), 853 (s), 834 (w), 769 (m), 739 (s), 702 (s), 675 (m), 640 (m), $597(w), 555(s), 534(w), 510(w), 482(w), 427(m)$.

M.p. $130^{\circ} \mathrm{C}$.

Anal. Calc. for $\mathrm{C}_{46} \mathrm{H}_{38} \mathrm{BF}_{10} \mathrm{P}: \mathrm{C}: 67.17 ; \mathrm{H}: 4.66$. Found: C: $66.79 ; \mathrm{H}: 5.02$.

A solution of the white solid in dichloromethane- $d_{2}$ showed at $213 \mathrm{~K}$ a mixture of two isomers 11 and $11^{\prime}$ [ratio ca. $77: 23\left({ }^{31} \mathrm{P}\right)$ ].

Major component (11):

${ }^{1} \mathbf{H}$ NMR $\left(500 \mathrm{MHz}\right.$, dichloromethane- $\left.d_{2}, 213 \mathrm{~K}\right): \delta 7.29(\mathrm{~m}, 2 \mathrm{H}, m-\mathrm{Ph}), 7.22(\mathrm{~m}, 2 \mathrm{H}, o-\mathrm{Ph})$, $7.19(\mathrm{~m}, 1 \mathrm{H}, p-\mathrm{Ph}), 7.00\left(\mathrm{~d},{ }^{4} J_{\mathrm{PH}}=3.2 \mathrm{~Hz}, 1 \mathrm{H}, m-\mathrm{Mes}^{\mathrm{a}}\right), 6.79\left(\mathrm{~m}, 1 \mathrm{H}, m-\mathrm{Mes}^{\mathrm{b}}\right), 6.78(\mathrm{~m}, 1 \mathrm{H}$, $\left.m^{\prime}-\mathrm{Mes}^{\mathrm{a}}\right), 6.51\left(\mathrm{~m}, 1 \mathrm{H}, m^{\prime}-\mathrm{Mes}^{\mathrm{b}}\right), 5.88(\mathrm{br}, 1 \mathrm{H}, 3-\mathrm{CH}), 4.14(\mathrm{br} \mathrm{d}, J=10.3 \mathrm{~Hz}, 1 \mathrm{H}, 4-\mathrm{CH}), 2.62$ (s, 3H, o- $\mathrm{CH}_{3}{ }^{\mathrm{Mes}, \mathrm{b}}$ ), $2.45\left(\mathrm{~s}, 3 \mathrm{H}, o-\mathrm{CH}_{3}{ }^{\mathrm{Mes}, \mathrm{a}}\right), 2.34(\mathrm{~m}, 1 \mathrm{H}, 5-\mathrm{CH}), 2.26\left(\mathrm{~s}, 3 \mathrm{H}, p-\mathrm{CH}_{3}{ }^{\mathrm{Mes}, \mathrm{a}}\right), 2.15$ (s, 3H, p- $\left.\mathrm{CH}_{3}{ }^{\mathrm{Mes}, \mathrm{b}}\right), 2.09(2 \mathrm{H}), 1.47(1 \mathrm{H}), 1.07(1 \mathrm{H}), 1.29(1 \mathrm{H}), 0.63(1 \mathrm{H}), 1.17(1 \mathrm{H}), 0.81$ $(1 \mathrm{H})\left(\right.$ each m, $\left.\mathrm{CH}_{2}\right), 1.96\left(\mathrm{~s}, 3 \mathrm{H}, o^{\prime}-\mathrm{CH}_{3}{ }^{\mathrm{Mes}, \mathrm{b}}\right), 1.83\left(\mathrm{~s}, 3 \mathrm{H}, o^{\prime}-\mathrm{CH}_{3}{ }^{\mathrm{Mes}, \mathrm{a}}\right)$.

${ }^{13} \mathrm{C}\left\{{ }^{1} \mathrm{H}\right\}$ NMR $\left(126 \mathrm{MHz}\right.$, dichloromethane- $\left.d_{2}, 213 \mathrm{~K}\right): \delta 153.1(\mathrm{br} \mathrm{dm}, J=3.8 \mathrm{~Hz}, 10-\mathrm{C}) \mathrm{t}, 145.7$ $(\mathrm{br}, 2-\mathrm{C})^{\mathrm{t}}, 143.8\left(\mathrm{~d},{ }^{2} \mathrm{JPC}_{\mathrm{PC}}=19.1 \mathrm{~Hz}, o-\mathrm{Mes}^{\mathrm{a}}\right), 142.6(i-\mathrm{Ph})^{t}, 142.6\left(\mathrm{~d},{ }^{2} J_{\mathrm{PC}}=6.5 \mathrm{~Hz}, o-\mathrm{Mes}^{\mathrm{b}}\right)$, $142.0\left(\mathrm{~d},{ }^{2} \mathrm{~J}_{\mathrm{PC}}=6.5 \mathrm{~Hz}, o^{\prime}-\mathrm{Mes}^{\mathrm{a}}\right), 141.7\left(\mathrm{~d},{ }^{2} \mathrm{~J}_{\mathrm{PC}}=14.9 \mathrm{~Hz}, o^{\prime}-\mathrm{Mes}^{\mathrm{b}}\right), 141.0\left(\mathrm{~d},{ }^{4} J_{\mathrm{PC}}=3.1 \mathrm{~Hz}, p-\right.$ $\left.\mathrm{Mes}^{\mathrm{a}}\right), 140.7\left(\mathrm{~d},{ }^{4} J_{\mathrm{PC}}=2.1 \mathrm{~Hz}, p-\mathrm{Mes}^{\mathrm{b}}\right), 131.2\left(\mathrm{~d},{ }^{3} J_{\mathrm{PC}}=7.2 \mathrm{~Hz}, m^{\prime}-\mathrm{Mes}^{\mathrm{a}}\right), 130.3\left(\mathrm{~d},{ }^{3} J_{\mathrm{PC}}=\right.$ 11.2 Hz, $m-\mathrm{Mes}^{\mathrm{a}}$ ), 130.1 (d, ${ }^{3} \mathrm{JPC}_{\mathrm{PC}}=7.8 \mathrm{~Hz}, m-\mathrm{Mes}^{\mathrm{b}}$ ), $128.6(o-\mathrm{Ph}), 128.3\left(\mathrm{~d},{ }^{3} \mathrm{JPC}_{\mathrm{PC}}=9.9 \mathrm{~Hz}, m^{\prime}-\right.$ Mes $\left.^{b}\right), 127.8(m-P h), 126.0$ (d, $\left.{ }^{1} J_{P C}=53.5 \mathrm{~Hz}, 1-C\right), 125.8(p-P h), 124.7$ (br d, J = 42.7 Hz 3$\mathrm{CH}), 123.8\left(\mathrm{~d},{ }^{1} \mathrm{~J}_{\mathrm{PC}}=41.9 \mathrm{~Hz}, i-\mathrm{Mes}^{\mathrm{b}}\right), 121.7\left(\mathrm{~d},{ }^{1} \mathrm{~J}_{\mathrm{PC}}=25.6 \mathrm{~Hz}, i-\mathrm{Mes}^{\mathrm{a}}\right), 47.9\left(\mathrm{~d},{ }^{3} J_{\mathrm{PC}}=10.9 \mathrm{~Hz}\right.$, 
5-CH), 45.6 (4-CH), $33.5(\mathrm{dm}, J=6.8 \mathrm{~Hz}), 27.8,27.6,25.8\left(\mathrm{CH}_{2}\right), 25.4\left(\mathrm{~d},{ }^{3} \mathrm{JPC}_{\mathrm{PC}}=10.5 \mathrm{~Hz}, o_{-}\right.$ $\left.\mathrm{CH}_{3}{ }^{\mathrm{Mes}, \mathrm{a}}\right), 24.3\left(\mathrm{~d},{ }^{3} \mathrm{JPC}_{\mathrm{PC}}=3.3 \mathrm{~Hz}\right.$, o' $\left.^{\prime} \mathrm{CH}_{3}{ }^{\mathrm{Mes}, \mathrm{a}}\right), 22.9\left(\mathrm{~m}, \mathrm{o}^{-} \mathrm{CH}_{3}{ }^{\mathrm{Mes}, \mathrm{b}}\right), 20.7$ ( $\left.\mathrm{m}, o^{\prime}-\mathrm{CH}_{3}{ }^{\mathrm{Mes}, \mathrm{b}}\right), 20.6$ (d, $\left.J=1.4 \mathrm{~Hz}, p-\mathrm{CH}_{3}{ }^{\text {Mes,a }}\right), 20.3\left(\mathrm{~d}, J=1.1 \mathrm{~Hz}, p-\mathrm{CH}_{3}{ }^{\mathrm{Mes}, \mathrm{b}}\right.$ ). $\left[\mathrm{C}_{6} \mathrm{~F}_{5}\right.$ not listed, ${ }^{t}$ tentatively assigned].

${ }^{31} \mathbf{P}\left\{{ }^{1} \mathrm{H}\right\}$ NMR $\left(202 \mathrm{MHz}\right.$, dichloromethane- $\left.d_{2}, 213 \mathrm{~K}\right): \delta 10.4\left(v_{1 / 2} \sim 40 \mathrm{~Hz}\right)$

${ }^{11} \mathrm{~B}\left\{{ }^{1} \mathrm{H}\right\}$ NMR (160 MHz, dichloromethane- $\left.d_{2}, 213 \mathrm{~K}\right): \delta 3.6$ (broad).

${ }^{11} \mathbf{B}\left\{{ }^{1} \mathrm{H}\right\}$ NMR $\left(160 \mathrm{MHz}\right.$, dichloromethane- $\left.d_{2}, 299 \mathrm{~K}\right): \delta 6.4\left(v_{1 / 2} \sim 550 \mathrm{~Hz}\right)$

${ }^{19} \mathrm{~F}$ NMR $\left(470 \mathrm{MHz}\right.$, dichloromethane- $\left.d_{2}, 213 \mathrm{~K}\right): \delta-124.5,-126.0,-129.3,-130.1$ (each br, each $\left.1 F, o-C_{6} F_{5}\right),-158.4\left(b r t,{ }^{3} J_{F F}=19.7 \mathrm{~Hz}\right),-159.5\left(b r t,{ }^{3} J_{\mathrm{FF}}=20.8 \mathrm{~Hz}\right)\left(\right.$ each $\left.1 F, p-C_{6} F_{5}\right)$, 164.6, -165.6 (each $\mathrm{m}$, each $2 \mathrm{~F}, m-\mathrm{C}_{6} \mathrm{~F}_{5}$ ).

Minor component (11'):

${ }^{1} \mathbf{H}$ NMR $\left(500 \mathrm{MHz}\right.$, dichloromethane- $\left.d_{2}, 213 \mathrm{~K}\right): \delta 7.20(\mathrm{~m}, 2 \mathrm{H}, m-\mathrm{Ph}), 7.16(\mathrm{~m}, 2 \mathrm{H}, \mathrm{o}-\mathrm{Ph})$, $7.14(\mathrm{~m}, 1 \mathrm{H}, p-\mathrm{Ph}), 7.01\left(\mathrm{br}, 1 \mathrm{H}, m-\mathrm{Mes}^{\mathrm{a}}\right), 6.94\left(\mathrm{br}, 1 \mathrm{H}, m-\mathrm{Mes}^{\mathrm{b}}\right), 6.80\left(\mathrm{br}, 1 \mathrm{H}, m^{\prime}-\mathrm{Mes}^{\mathrm{a}}\right), 6.47$ (br, 1H, m'-Mes ${ }^{b}$ ), $5.92(\mathrm{br}, 1 \mathrm{H}, 3-\mathrm{CH}), 3.47$ (br m, 1H, 4-CH), $2.78(\mathrm{~m}, 1 \mathrm{H}, 5-\mathrm{CH}), 2.76(\mathrm{~s}, 3 \mathrm{H}$, $o-\mathrm{CH}_{3}{ }^{\mathrm{Mes}, \mathrm{b}}$ ), 2.27 (s, 3H, $p-\mathrm{CH}_{3}{ }^{\mathrm{Mes}, \mathrm{a}}$ ), 2.21 (s, 3H, o- $\mathrm{CH}_{3}{ }^{\mathrm{Mes}, \mathrm{a}}$ ), $2.18\left(\mathrm{~s}, 3 \mathrm{H}, p-\mathrm{CH}_{3}{ }^{\mathrm{Mes}, \mathrm{b}}\right.$ ), $2.14(1 \mathrm{H})$, $1.61(1 \mathrm{H}), 1.48(1 \mathrm{H}), 1.46(1 \mathrm{H}), 1.27(1 \mathrm{H}), 1.14(1 \mathrm{H}), 1.05(1 \mathrm{H}), 0.94(1 \mathrm{H})\left(\right.$ each $\left.m, \mathrm{CH}_{2}\right), 1.98$ (s, 3H, o'- $\left.\mathrm{CH}_{3}{ }^{\text {Mes,b}}\right), 1.76$ (s, 3H, o'- $\left.\mathrm{CH}_{3}{ }^{\text {Mes,a }}\right)$.

${ }^{13} \mathrm{C}\left\{{ }^{1} \mathrm{H}\right\}$ NMR $\left(126 \mathrm{MHz}\right.$, dichloromethane- $\left.d_{2}, 213 \mathrm{~K}\right): \delta 149.8(\mathrm{br} \mathrm{d}, J=.4 .2 \mathrm{~Hz}, 10-\mathrm{C}), 144.2$ $\left(\mathrm{d},{ }^{2} \mathrm{JPC}_{\mathrm{PC}}=20.0 \mathrm{~Hz}, \mathrm{o}-\mathrm{Mes}^{\mathrm{a}}\right), 142.8\left(\mathrm{~m}, \mathrm{o}_{\mathrm{Mes}}{ }^{\mathrm{b}}\right), 142.6(i-\mathrm{Ph})^{t}, 142.2\left(\mathrm{~m}, o^{\prime}-\mathrm{Mes}^{\mathrm{b}}\right), 142.0(\mathrm{~m}, p-$ $\left.\operatorname{Mes}^{\mathrm{a}}\right), 141.9\left(\mathrm{~d},{ }^{2} J_{\mathrm{PC}}=6.5 \mathrm{~Hz}, o^{\prime}-\mathrm{Mes}^{\mathrm{a}}\right), 141.1\left(\mathrm{~m}, p-\mathrm{Mes}^{\mathrm{b}}\right), 131.7\left(\mathrm{~d},{ }^{3} J_{\mathrm{PC}}=8.2 \mathrm{~Hz}, m^{\prime}-\mathrm{Mes}^{\mathrm{a}}\right)$, $130.2\left(\mathrm{~d},{ }^{3} \mathrm{JPC}_{\mathrm{PC}}=10.1 \mathrm{~Hz}, m-\mathrm{Mes}^{\mathrm{a}}\right), 129.9\left(\mathrm{~d},{ }^{3} \mathrm{JPC}_{\mathrm{PC}}=8.2 \mathrm{~Hz}, m-\mathrm{Mes}^{\mathrm{b}}\right), 128.7(o-\mathrm{Ph}), 128.4(\mathrm{~d}$, $\left.{ }^{1} J_{\mathrm{PC}}=53.2 \mathrm{~Hz}, 1-\mathrm{C}\right), 128.0\left(\mathrm{~m}, m^{\prime}-\mathrm{Mes}^{\mathrm{b}}\right), 128.0(m-\mathrm{Ph}), 126.2(\mathrm{br}, 3-\mathrm{CH}), 125.8(p-\mathrm{Ph}), 123.6$ $\left(\mathrm{d},{ }^{1} J_{\mathrm{PC}}=41.6 \mathrm{~Hz}, i-\mathrm{Mes}^{\mathrm{b}}\right), 120.3\left(\mathrm{~d},{ }^{1} J_{\mathrm{PC}}=26.6 \mathrm{~Hz}, i-\mathrm{Mes}^{\mathrm{a}}\right), 45.4(\mathrm{br}, 4-\mathrm{CH}), 39.8\left(\mathrm{~d},{ }^{3} J_{\mathrm{PC}}=\right.$ $9.6 \mathrm{~Hz}, 5-\mathrm{CH}), 31.7\left(\mathrm{~d},{ }^{3} \mathrm{JPC}_{\mathrm{PC}}=8.2 \mathrm{~Hz}\right), 28.5,25.2,23.3\left(\mathrm{CH}_{2}\right), 24.2\left(\mathrm{~d},{ }^{3} \mathrm{JPC}_{\mathrm{PC}}=3.3 \mathrm{~Hz}, o^{\prime}-\right.$ $\left.\mathrm{CH}_{3}{ }^{\mathrm{Mes}, \mathrm{a}}\right), 24.2\left(\mathrm{~d},{ }^{3} \mathrm{~J}_{\mathrm{PC}}=9.0 \mathrm{~Hz}, \mathrm{o}-\mathrm{CH}_{3}{ }^{\mathrm{Mes}, \mathrm{a}}\right), 21.4\left(\mathrm{~m}, \mathrm{o}-\mathrm{CH}_{3}{ }^{\mathrm{Mes}, \mathrm{b}}\right), 20.7\left(\mathrm{~m}, \mathrm{o}^{\prime}-\mathrm{CH}_{3}{ }^{\mathrm{Mes}, \mathrm{b}}\right), 20.6$ (m, $\left.p-\mathrm{CH}_{3}{ }^{\mathrm{Mes}, \mathrm{a}}\right), 20.4\left(p-\mathrm{CH}_{3}{ }^{\mathrm{Mes}, \mathrm{b}}\right)$, n.o. 2-C. $\left[\mathrm{C}_{6} \mathrm{~F}_{5}\right.$ not listed, ${ }^{t}$ tentatively assigned].

${ }^{31} \mathbf{P}\left\{{ }^{1} \mathrm{H}\right\}$ NMR $\left(202 \mathrm{MHz}\right.$, dichloromethane- $\left.d_{2}, 213 \mathrm{~K}\right): \delta 12.7$ (partial relaxed br 1:1:1:1 q, ${ }^{1} \mathrm{JPB}_{\mathrm{PB}}$ $\sim 15 \mathrm{~Hz})$

${ }^{11} \mathrm{~B}\left\{{ }^{1} \mathrm{H}\right\}$ NMR (160 MHz, dichloromethane- $\left.d_{2}, 213 \mathrm{~K}\right): \delta 3.6$ (broad).

${ }^{11} \mathbf{B}\left\{{ }^{1} \mathrm{H}\right\}$ NMR $\left(160 \mathrm{MHz}\right.$, dichloromethane- $\left.d_{2}, 299 \mathrm{~K}\right): \delta 6.4\left(v_{1 / 2} \sim 550 \mathrm{~Hz}\right)$

${ }^{19} \mathrm{~F}$ NMR $\left(470 \mathrm{MHz}\right.$, dichloromethane- $\left.d_{2}, 213 \mathrm{~K}\right): \delta-121.7,-127.4,-129.9,-130.1$ (each m, each $\left.1 \mathrm{~F}, o-\mathrm{C}_{6} \mathrm{~F}_{5}\right),-158.1\left(\mathrm{br} t,{ }^{3} J_{\mathrm{FF}}=19.8 \mathrm{~Hz}\right),-159.6\left(\mathrm{t},{ }^{3} \mathrm{~J}_{\mathrm{FF}}=20.2 \mathrm{~Hz}\right)\left(\right.$ each $\left.1 \mathrm{~F}, p-\mathrm{C}_{6} \mathrm{~F}_{5}\right)$, $164.5(2 \mathrm{~F}),-165.4(1 \mathrm{~F}), 165.5(1 \mathrm{~F})\left(\right.$ each $\left.\mathrm{m}, \mathrm{m}-\mathrm{C}_{6} \mathrm{~F}_{5}\right)$. 

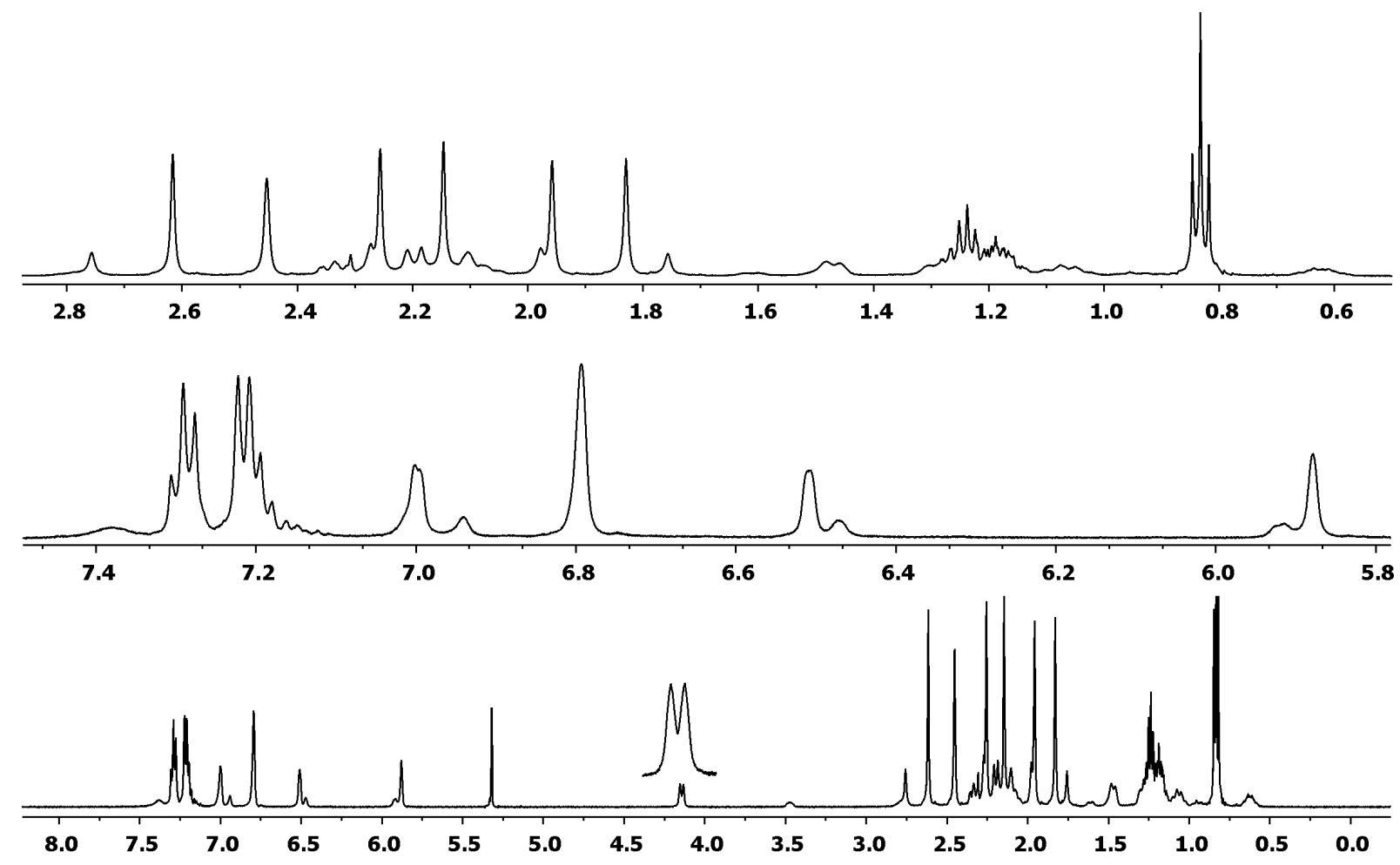

Figure S19: ${ }^{1} \mathrm{H}$ NMR $\left(500 \mathrm{MHz}\right.$, dichloromethane- $\left.d_{2}, 213 \mathrm{~K}\right)$ spectrum of compound 11 [admixed with pentane]
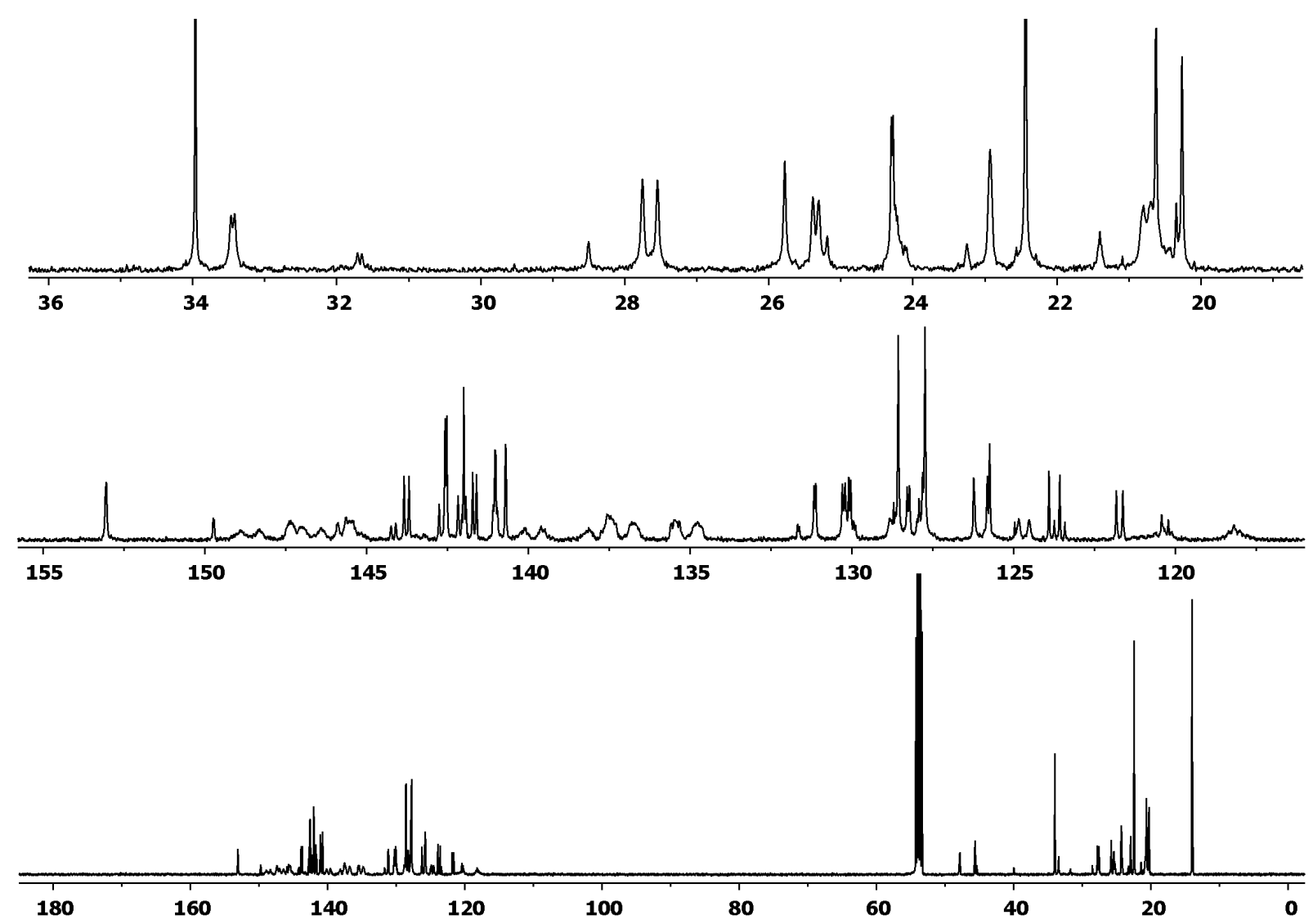

Figure S20: ${ }^{13} \mathbf{C}\left\{{ }^{1} \mathbf{H}\right\}$ NMR $(126 \mathrm{MHz}$, dichloromethane-d2, $213 \mathrm{~K})$ spectrum of compound $\mathbf{1 1}$ [admixed with pentane] 


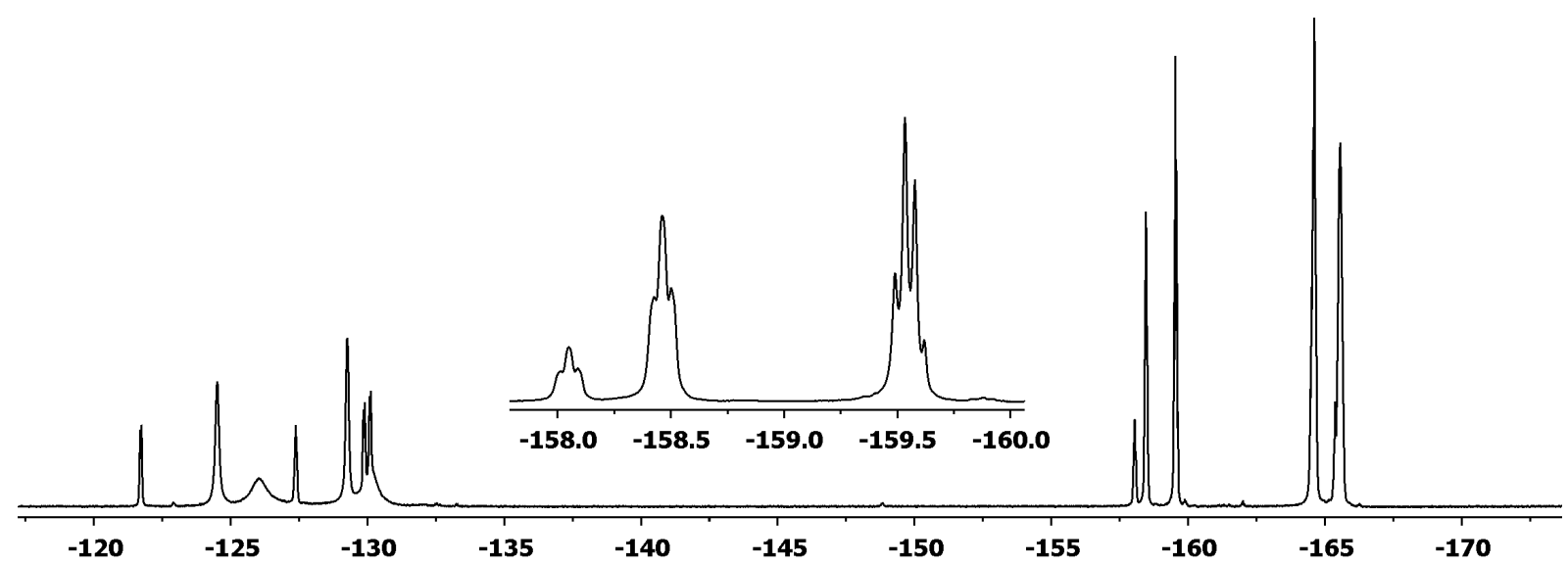

Figure S21: ${ }^{19} \mathrm{~F}$ NMR (470 MHz, dichloromethane- $\left.d_{2}, 213 \mathrm{~K}\right)$ spectrum of compound 11

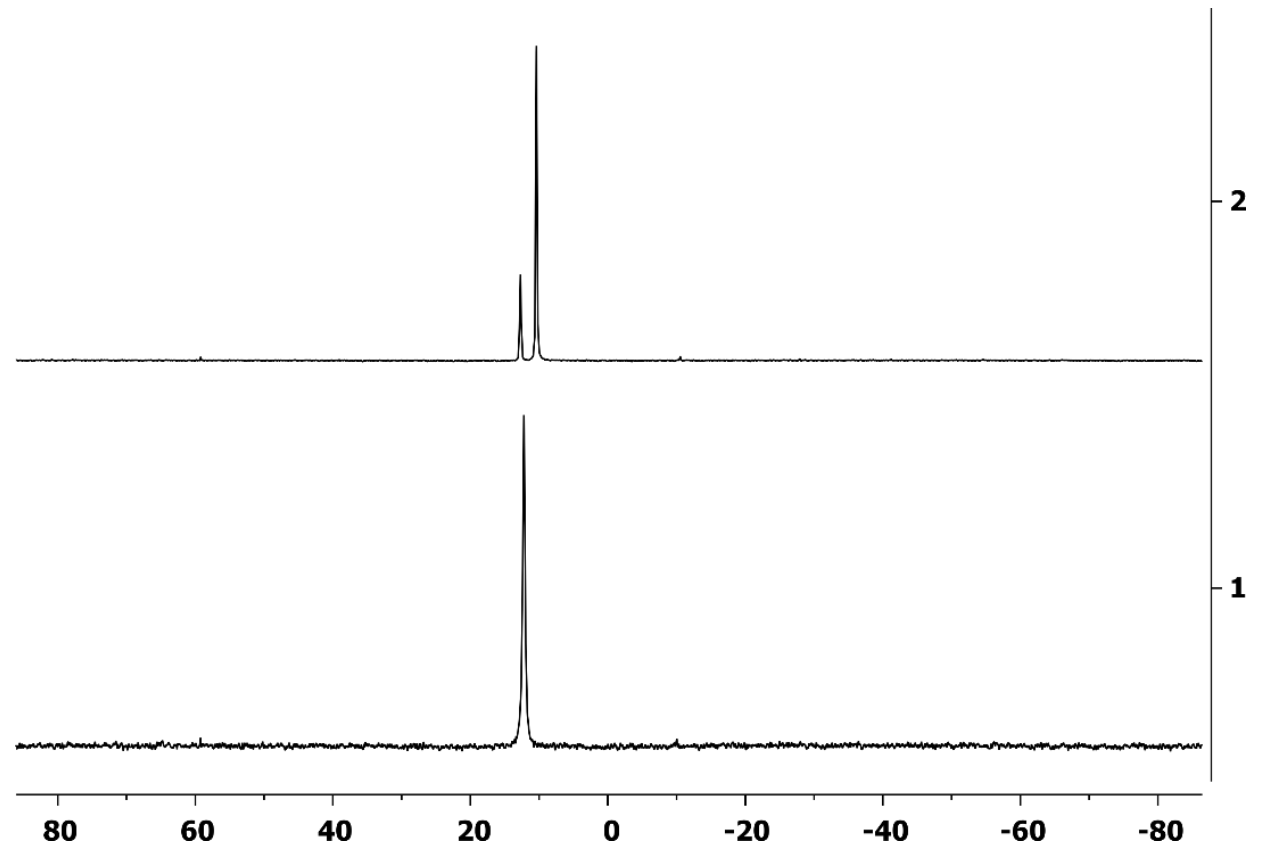

Figure S22: ${ }^{31} \mathrm{P}\left\{{ }^{1} \mathrm{H}\right\}$ NMR (202 MHz, dichloromethane- $\left.d_{2}\right)$ spectra of compound 11 at $299 \mathrm{~K}(1)$ and $213 \mathrm{~K}(2)$.

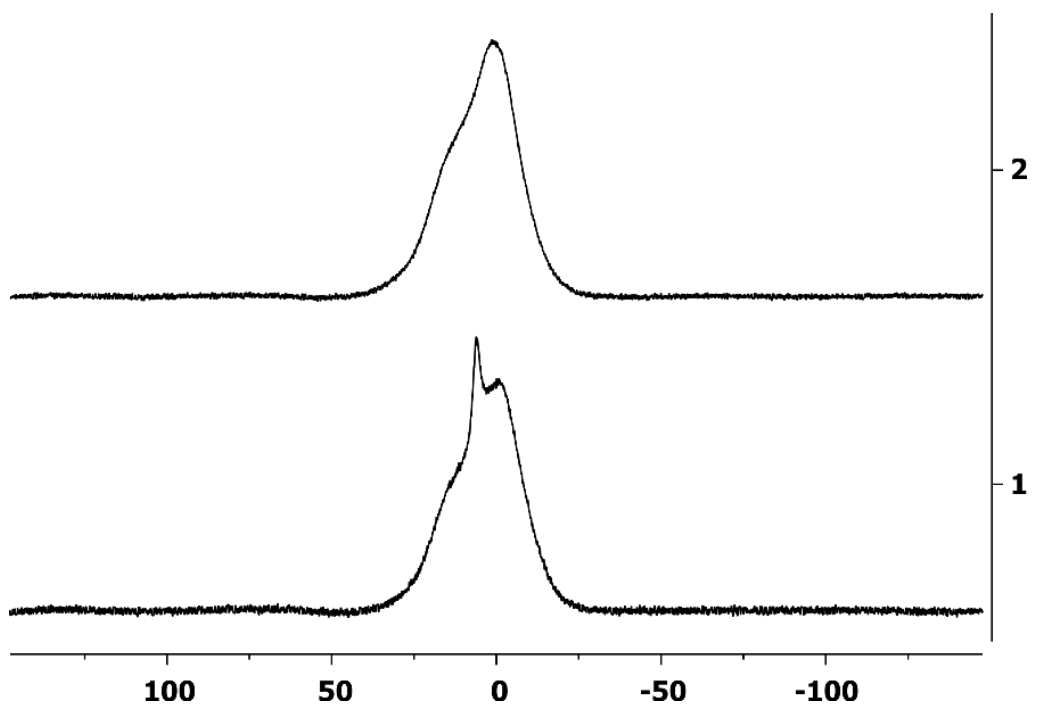

Figure S23: ${ }^{11} \mathrm{~B}\left\{{ }^{1} \mathrm{H}\right\}$ NMR (160 MHz, dichloromethane- $\left.d_{2}\right)$ spectra of compound 11 at $299 \mathrm{~K}(1)$ and $213 \mathrm{~K}(2)$ 


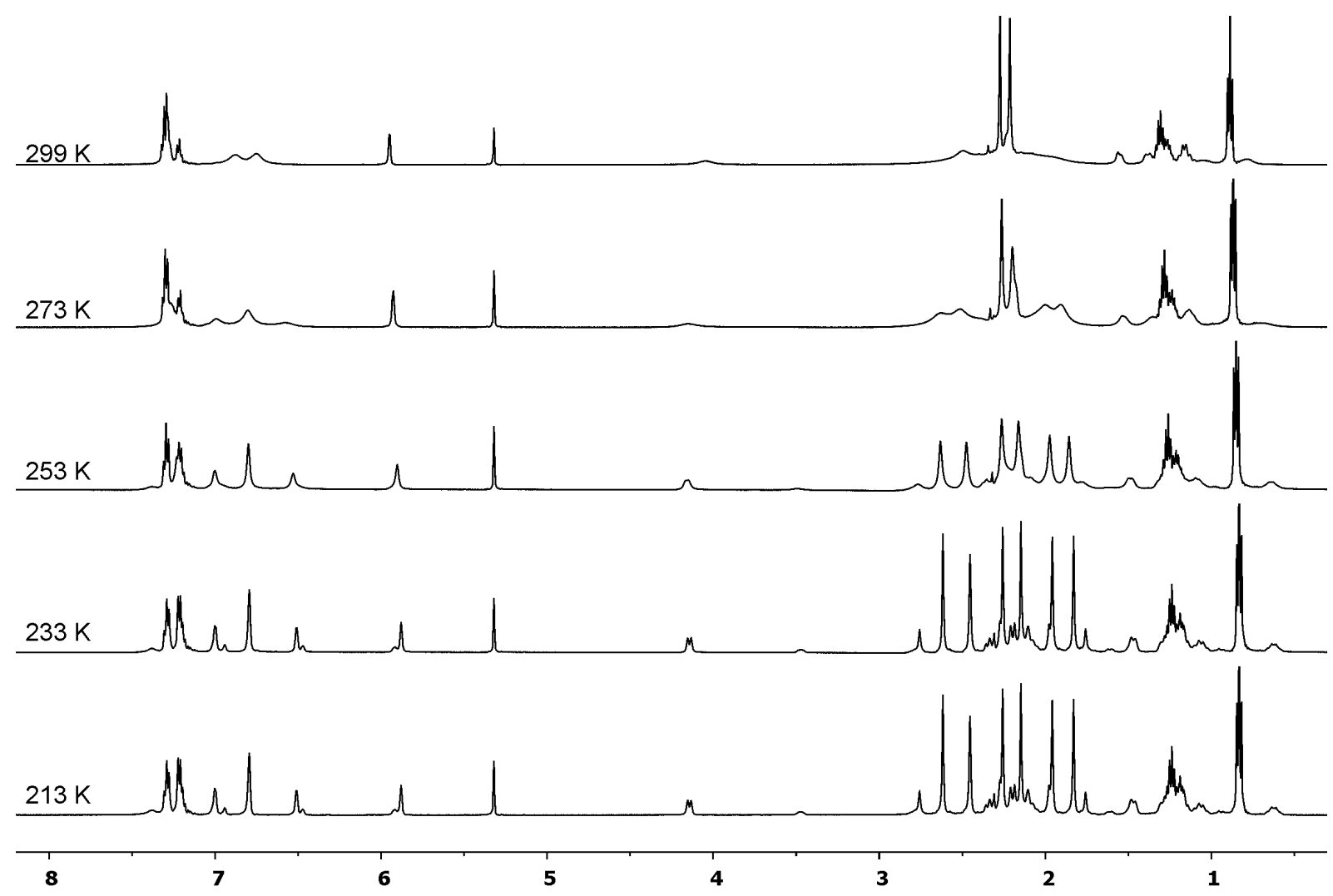

Figure S24: Dynamic ${ }^{1} \mathrm{H}$ NMR ( $500 \mathrm{MHz}$, dichloromethane- $d_{2}$ ) spectra of compound 11 [admixed with pentane]

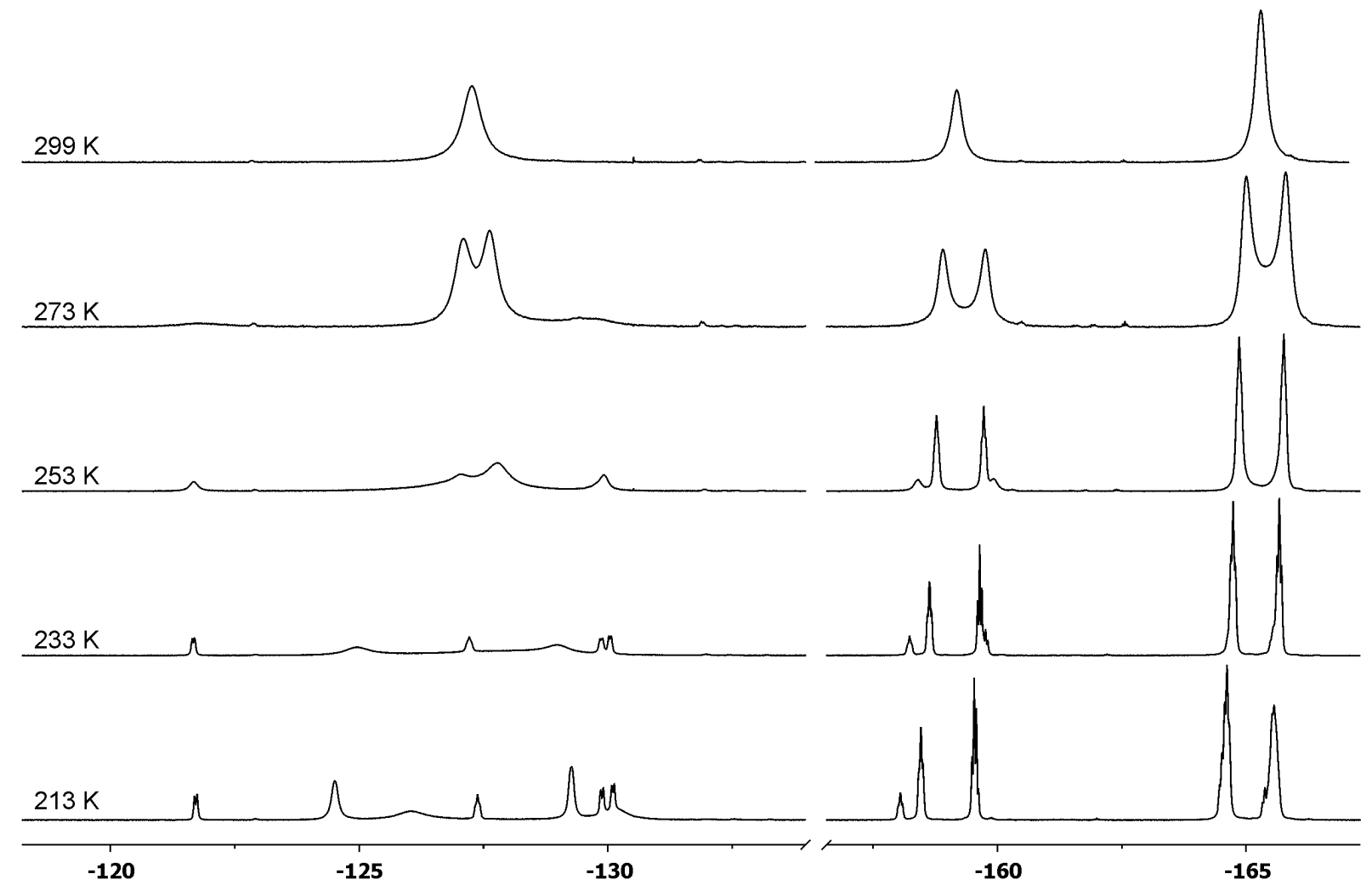

Figure S25: Dynamic ${ }^{19} \mathrm{~F}$ NMR $\left(470 \mathrm{MHz}\right.$, dichloromethane- $\left.d_{2}\right)$ spectra of compound 11 
$\Delta \mathrm{G}^{\ddagger}\left[\mathrm{T}_{\mathrm{c}}, \Delta v(\mathrm{~T})\right]=\mathrm{RT}_{\mathrm{c}}(22.96+\ln (\mathrm{Tc} / \Delta \mathrm{v}))[\mathrm{J} / \mathrm{mol}]$

$\mathrm{T}_{\mathrm{c}}=$ coalescence temperature $[\mathrm{K}]: 286 \mathrm{~K}\left({ }^{19} \mathrm{~F}, p-\mathrm{BC}_{6} \mathrm{~F}_{5}\right)$

$\Delta v=$ chemical shift difference $[\mathrm{Hz}]:\left({ }^{19} \mathrm{~F}, p-\mathrm{BC}_{6} \mathrm{~F}_{5}, 233 \mathrm{~K}\right): 476 \mathrm{~Hz}$

$\mathrm{R}=8.314 \mathrm{~J} /(\mathrm{mol} \cdot \mathrm{K}) ; 1 \mathrm{~J}=0.239 \mathrm{cal}$

$\Delta \mathrm{G}^{\ddagger}[286 \mathrm{~K}, \Delta v(233 \mathrm{~K})=476 \mathrm{~Hz}]=53.383 \mathrm{~J} / \mathrm{mol}=12.8 \pm 0.3 \mathrm{kcal} / \mathrm{mol}$

Crystals suitable for the X-ray crystal structure analysis were obtained by slow diffusion of pentane into a solution of compound 11 in dichloromethane at $-35^{\circ} \mathrm{C}$ :

X-ray crystal structure analysis of compound 11: A colorless prism-like specimen of $\mathrm{C}_{46} \mathrm{H}_{38} \mathrm{BF}_{10} \mathrm{P} \cdot \mathrm{C}_{5} \mathrm{H}_{12}$, approximate dimensions $0.050 \mathrm{~mm} \times 0.100 \mathrm{~mm} \times 0.120 \mathrm{~mm}$, was used for the X-ray crystallographic analysis. The X-ray intensity data were measured. A total of 346 frames were collected. The total exposure time was 4.33 hours. The frames were integrated with the Bruker SAINT software package using a narrow-frame algorithm. The integration of the data using a monoclinic unit cell yielded a total of 48383 reflections to a maximum $\theta$ angle of $25.35^{\circ}$ ( $0.83 \AA$ resolution), of which 7874 were independent (average redundancy 6.145, completeness $\left.=99.9 \%, R_{\text {int }}=10.95 \%, R_{\text {sig }}=6.65 \%\right)$ and $5407(68.67 \%)$ were greater than $2 \sigma\left(F^{2}\right)$. The final cell constants of $\underline{a}=14.4106(7) \AA$, $\underline{b}=21.7070(9) \AA, \underline{c}=14.5170(5) \AA, \beta=$ $108.8030(10)^{\circ}$, volume $=4298.7(3) \AA^{3}$, are based upon the refinement of the $X Y Z$-centroids of 9539 reflections above $20 \sigma(\mathrm{I})$ with $4.782^{\circ}<2 \theta<52.77^{\circ}$. Data were corrected for absorption effects using the multi-scan method (SADABS). The ratio of minimum to maximum apparent transmission was 0.943 . The calculated minimum and maximum transmission coefficients (based on crystal size) are 0.9830 and 0.9930 . The structure was solved and refined using the Bruker SHELXTL Software Package, using the space group P $121 / n 1$, with $Z=4$ for the formula unit, $\mathrm{C}_{46} \mathrm{H}_{38} \mathrm{BF}_{10} \mathrm{P} \cdot \mathrm{C}_{5} \mathrm{H}_{12}$. The final anisotropic full-matrix least-squares refinement on $F^{2}$ with 620 variables converged at $R 1=5.00 \%$, for the observed data and $w R 2=10.32 \%$ for all data. The goodness-of-fit was 1.027. The largest peak in the final difference electron density synthesis was $0.256 \mathrm{e}^{-} / \AA^{3}$ and the largest hole was $-0.255 \mathrm{e}^{-} / \AA^{3}$ with an RMS deviation of 0.059 $\mathrm{e}^{-} / \AA^{3}$. On the basis of the final model, the calculated density was $1.382 \mathrm{~g} / \mathrm{cm}^{3}$ and $F(000)$, $1864 \mathrm{e}^{-}$. 


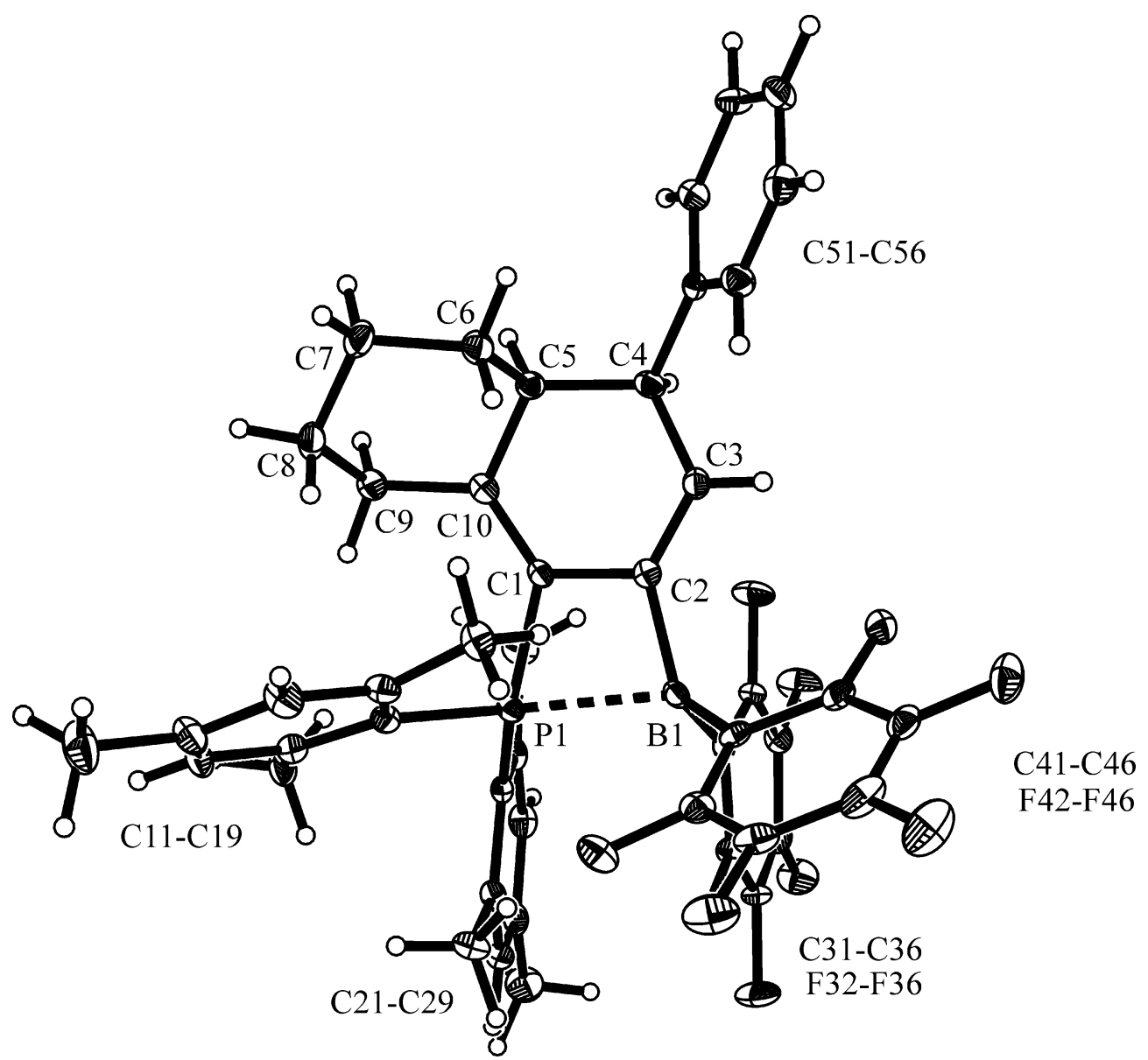

Figure S26: X-ray crystal structure of compound 11 (thermal ellipsoids are shown at the $50 \%$ probability level)

\section{Synthesis of compound 12}

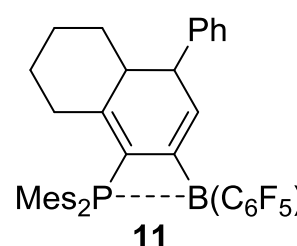

$$
\frac{2 \text { eq TEMPO }}{60{ }^{\circ} \mathrm{C}, \mathrm{C}_{6} \mathrm{H}_{6}}
$$<smiles>C[PH](c1c(Br)cc(-c2ccccc2)c2c1CCCC2)(C(F)(F)F)C(F)(F)F</smiles>

Scheme S5

A solution of TEMPO (152.0 mg, $972.6 \mu \mathrm{mol}, 2.0 \mathrm{eq})$ in benzene $(2 \mathrm{~mL})$ was added to a solution of compound 11 (400.0 mg, $486.3 \mu \mathrm{mol}, 1.00 \mathrm{eq})$ in benzene ( $3 \mathrm{~mL}$ ). The reaction mixture was stirred at $60^{\circ} \mathrm{C}$ for 2 days. Then all volatiles were removed in vacuo and the resulting sticky red residue was dissolved in pentane $(3 \mathrm{~mL})$, which was subsequently removed in vacuo. After addition of pentane $(3 \mathrm{~mL})$ to the obtained residue the resulting suspension was stored at $-35^{\circ} \mathrm{C}$ for 1 day. Subsequently the off-white solid material was collected and washed with cold pentane $(5 \times 2 \mathrm{~mL}$ ) to yield compound 12 as a white fluffy solid (319.2 $\mathrm{mg}, 0.4 \mathrm{mmol}$, $80 \%)$. 
IR $(\mathrm{KBr}): \tilde{v}\left[\mathrm{~cm}^{-1}\right]=3688(\mathrm{~m}), 3016(\mathrm{w}), 3028(\mathrm{w}), 2935(\mathrm{~m}), 2860(\mathrm{~m}), 2773(\mathrm{w}), 2739(\mathrm{w})$, $2473(w), 2399$ (w), 2348 (w), 2314 (w), 1962 (w), $1886(w), 1812(w), 1735$ (w), 1642 (s), 1603 (s), $1559(\mathrm{~m}), 1515(\mathrm{~s}), 1464(\mathrm{~m}), 1381(\mathrm{~m}), 1324(\mathrm{w}), 1305(\mathrm{~m}), 1270(\mathrm{~m}), 1260(\mathrm{~m}), 1201(\mathrm{w})$, $1178(\mathrm{~m}), 1160(\mathrm{~m}), 1092(\mathrm{~s}), 1030(\mathrm{~m}), 962(\mathrm{~s}), 894(\mathrm{~m}), 851(\mathrm{~s}), 771(\mathrm{~s}), 704(\mathrm{~s}), 678(\mathrm{~m})$, $637(m), 574(w), 554(m), 509(w), 473(w), 440(m), 408(w)$.

M.p. $136^{\circ} \mathrm{C}$.

Anal. Calc. for $\mathrm{C}_{46} \mathrm{H}_{36} \mathrm{BF}_{10} \mathrm{P}: \mathrm{C}: 67.33 ; \mathrm{H}: 4.42$. Found: C: $67.65 ; \mathrm{H}: 5.17$.

${ }^{1} \mathbf{H}$ NMR $\left(500 \mathrm{MHz}\right.$, dichloromethane- $\left.d_{2}, 299 \mathrm{~K}\right): \delta 7.42(3 \mathrm{H}), 7.34(3 \mathrm{H})($ each $\mathrm{m}, \mathrm{Ph}, 3-\mathrm{CH})$, $6.81\left(\mathrm{~d},{ }^{4} \mathrm{~J}_{\mathrm{PH}}=3.2 \mathrm{~Hz}, 4 \mathrm{H}, \mathrm{m}\right.$-Mes), $2.55(2 \mathrm{H}), 2.37(2 \mathrm{H}), 1.60(4 \mathrm{H})$ (each br m, $\left.\mathrm{CH}_{2}\right), 2.25$ (s, $\left.6 \mathrm{H}, p-\mathrm{CH}_{3}{ }^{\mathrm{Mes}}\right), 2.09$ (s, $\left.12 \mathrm{H}, o-\mathrm{CH}_{3}{ }^{\mathrm{Mes}}\right)$.

${ }^{13} \mathrm{C}\left\{{ }^{1} \mathrm{H}\right\}$ NMR $\left(126 \mathrm{MHz}\right.$, dichloromethane- $\left.d_{2}, 299 \mathrm{~K}\right): \delta 156.6\left(\mathrm{br} \mathrm{d},{ }^{2} \mathrm{JPC}=30.2 \mathrm{~Hz}, 2-\mathrm{C}\right), 148.2$, $(\mathrm{d}, J=2.8 \mathrm{~Hz}) 138.9(\mathrm{~d}, J=0.9 \mathrm{~Hz}), 135.9(\mathrm{~d}, J=8.5 \mathrm{~Hz})(4,5,10-\mathrm{C})^{\mathrm{t}}, 148.0\left(\mathrm{dm},{ }^{1} J_{\mathrm{FC}} \sim 240 \mathrm{~Hz}\right.$, $\mathrm{C}_{6} \mathrm{~F}_{5}$ ), 142.7 (br d, $\left.{ }^{2} \mathrm{JPC}_{\mathrm{PC}}=8.9 \mathrm{~Hz}, 0-\mathrm{Mes}\right), 142.5(\mathrm{~d}, J=1.9 \mathrm{~Hz}, i-\mathrm{Ph}), 141.2\left(\mathrm{br} d,{ }^{4} J_{\mathrm{PC}}=2.9 \mathrm{~Hz}\right.$ p-Mes), $140.2\left(\mathrm{dm},{ }^{1} \mathrm{JFC}_{\mathrm{FC}} 250 \mathrm{~Hz}, \mathrm{C}_{6} \mathrm{~F}_{5}\right), 137.0\left(\mathrm{dm},{ }^{1} \mathrm{JFC}_{\mathrm{FC}} 250 \mathrm{~Hz}, \mathrm{C}_{6} \mathrm{~F}_{5}\right), 134.8\left(\mathrm{~d},{ }^{1} \mathrm{~J}_{\mathrm{PC}}=\right.$ $53.5 \mathrm{~Hz}, 1-\mathrm{C})^{\mathrm{t}}, 131.5(\mathrm{dm}, J=46.2 \mathrm{~Hz}, 3-\mathrm{CH}), 130.9$ (br d, $\left.{ }^{3} J_{\mathrm{PC}}=8.9 \mathrm{~Hz}, m-\mathrm{Mes}\right), 129.5,128.4$, $127.3(p)(\mathrm{Ph}), 127.4\left(\mathrm{br}, i-\mathrm{C}_{6} \mathrm{~F}_{5}\right), 124.5\left(\mathrm{br} \mathrm{dm},{ }^{1} \mathrm{PCC}_{\mathrm{PC}}=35.1 \mathrm{~Hz}, i-\mathrm{Mes}\right), 28.7(\mathrm{~d}, J=1.0 \mathrm{~Hz})$, $27.9(\mathrm{~d}, J=4.1 \mathrm{~Hz}), 23.5,22.4\left(\mathrm{CH}_{2}\right), 22.8\left(\mathrm{br} \mathrm{dm},{ }^{3} \mathrm{JPC}=5.4 \mathrm{~Hz}, o-\mathrm{CH}_{3}{ }^{\mathrm{Mes}}\right), 20.8(\mathrm{~d}, J=1.4 \mathrm{~Hz}$, $\left.p-\mathrm{CH}_{3}{ }^{\mathrm{Mes}}\right)$. [ ${ }^{\mathrm{t}}$ tentatively assigned].

${ }^{31} \mathbf{P}\left\{{ }^{1} \mathrm{H}\right\}$ NMR $\left(202 \mathrm{MHz}\right.$, dichloromethane- $\left.d_{2}, 299 \mathrm{~K}\right): \delta 5.8\left(v_{1 / 2} \sim 36 \mathrm{~Hz}\right)$.

${ }^{11} \mathbf{B}\left\{{ }^{1} \mathrm{H}\right\}$ NMR $\left(160 \mathrm{MHz}\right.$, dichloromethane- $\left.d_{2}, 299 \mathrm{~K}\right): \delta 5.7\left(v_{1 / 2} \sim 500 \mathrm{~Hz}\right)$.

${ }^{19} \mathrm{~F} \mathrm{NMR}\left(470 \mathrm{MHz}\right.$, dichloromethane- $\left.d_{2}, 299 \mathrm{~K}\right): \delta-128.1\left(\mathrm{br}, 2 \mathrm{~F}, \mathrm{o}-\mathrm{C}_{6} \mathrm{~F}_{5}\right),-159.1\left(\mathrm{t}, 1 \mathrm{~F},{ }^{3} J_{\mathrm{FF}}=\right.$ $\left.20.2 \mathrm{~Hz}, p-\mathrm{C}_{6} \mathrm{~F}_{5}\right),-165.5\left(\mathrm{~m}, 2 \mathrm{~F}, m-\mathrm{C}_{6} \mathrm{~F}_{5}\right)\left[\Delta \delta^{19} \mathrm{~F}_{\mathrm{mp}}=6.4\right]$.

${ }^{1} \mathrm{H}$ NMR $\left(500 \mathrm{MHz}\right.$, dichloromethane- $\left.d_{2}, 193 \mathrm{~K}\right): \delta 7.39(2 \mathrm{H}), 7.31(4 \mathrm{H})($ each $\mathrm{m}, \mathrm{Ph}, 3-\mathrm{CH})$, $6.94\left(\mathrm{~d},{ }^{4} \mathrm{~J}_{\mathrm{PH}}=2.3 \mathrm{~Hz}, 1 \mathrm{H}, m-\mathrm{Mes}^{\mathrm{a}}\right), 6.85\left(\mathrm{~s}, 1 \mathrm{H}, m^{\prime}-\mathrm{Mes}^{\mathrm{a}}\right), 6.66\left(\mathrm{~s}, 1 \mathrm{H}, m-\mathrm{Mes}^{\mathrm{b}}\right), 6.52(\mathrm{~d}$, $\left.{ }^{4} J_{\mathrm{PH}}=2.0 \mathrm{~Hz}, 1 \mathrm{H}, m^{\prime}-\mathrm{Mes}^{\mathrm{b}}\right), 2.55(2 \mathrm{H}), 2.43(1 \mathrm{H}), 1.97(1 \mathrm{H}), 1.61(1 \mathrm{H}), 1.52(2 \mathrm{H}), 1.38(1 \mathrm{H})$ (each br m, $\mathrm{CH}_{2}$ ), 2.25 (s, 3H, p- $\mathrm{CH}_{3}{ }^{\mathrm{Mes}, \mathrm{a}}$ ), 2.09 (s, 3H, $p-\mathrm{CH}_{3}{ }^{\mathrm{Mes}, \mathrm{b}}$ ), 2.06 (s, 3H, o'- $\mathrm{CH}_{3}{ }^{\mathrm{Mes}, \mathrm{b}}$ ), $2.03\left(\mathrm{~s}, 3 \mathrm{H}, \mathrm{o}-\mathrm{CH}_{3}{ }^{\mathrm{Mes}, \mathrm{b}}\right), 1.96\left(\mathrm{~s}, 3 \mathrm{H}, \mathrm{o}-\mathrm{CH}_{3}{ }^{\mathrm{Mes}, \mathrm{a}}\right), 1.83$ (s, 3H, o'- $\left.\mathrm{CH}_{3}{ }^{\mathrm{Mes}, \mathrm{a}}\right)$.

${ }^{13} \mathbf{C}\left\{{ }^{1} \mathrm{H}\right\}$ NMR $\left(126 \mathrm{MHz}\right.$, dichloromethane- $\left.d_{2}, 193 \mathrm{~K}\right): \delta 155.0\left(\mathrm{br} \mathrm{d},{ }^{2} \mathrm{JPC}=30.6 \mathrm{~Hz}, 2-\mathrm{C}\right), 146.8$, 137.7, $134.4(\mathrm{~d}, J=8.4 \mathrm{~Hz})(4,5,10-\mathrm{C})^{\mathrm{t}}, 143.3\left(\mathrm{~d},{ }^{2} \mathrm{JPC}=18.3 \mathrm{~Hz}, o-\mathrm{Mes}^{\mathrm{a}}\right), 142.1\left(o^{\prime}-\mathrm{Mes}^{\mathrm{a}}\right)$, $141.2(\mathrm{~m}, i-\mathrm{Ph}), 141.2$ (p-Mes $\left.{ }^{\mathrm{a}}\right), 141.1$ (d, $\left.{ }^{2} \mathrm{JPC}_{\mathrm{PC}}=16.0 \mathrm{~Hz}, o^{\prime}-\mathrm{Mes}^{\mathrm{b}}\right), 140.5\left(\right.$ o-Mes $\left.^{\mathrm{b}}\right), 140.2(\mathrm{~m}$, $\left.p-M^{b}{ }^{b}\right), 133.7\left(d,{ }^{1} J_{P C}=54.2 \mathrm{~Hz}, 1-C\right){ }^{t}, 131.4\left(\mathrm{br} \mathrm{d},{ }^{3} J_{\mathrm{PC}}=7.0 \mathrm{~Hz}, m^{\prime}-\mathrm{Mes}^{\mathrm{a}}\right), 130.6(\mathrm{dd}, \mathrm{J}=$ $45.0 \mathrm{~Hz}, J=12.0 \mathrm{~Hz}, 3-\mathrm{CH}), 130.0\left(\mathrm{br} d,{ }^{3} \mathrm{PCC}_{\mathrm{PC}}=10.7 \mathrm{~Hz}, m-\mathrm{Mes}^{\mathrm{a}}\right.$ ), 129.7 (d, ${ }^{3} \mathrm{JPC}_{\mathrm{PC}}=7.4 \mathrm{~Hz}, m-$ Mes $\left.^{b}\right), 128.8,128.4,127.8,127.5,126.6(p)$ (each br, Ph), $128.1\left(d,{ }^{3} J_{P C}=9.4 \mathrm{~Hz}, m^{\prime}-M_{e s}^{b}\right)$, $125.5\left(\mathrm{~d},{ }^{1} \mathrm{JPC}_{\mathrm{PC}}=40.3 \mathrm{~Hz}, i-\mathrm{Mes}^{\mathrm{b}}\right), 120.0\left(\mathrm{~d},{ }^{1} \mathrm{JPC}_{\mathrm{PC}}=28.5 \mathrm{~Hz}, i-\mathrm{Mes}^{\mathrm{a}}\right), 28.2,27.2(\mathrm{~d}, J=3.7 \mathrm{~Hz})$, 22.6, $21.5\left(\mathrm{CH}_{2}\right), 24.7\left(\mathrm{~d},{ }^{3} \mathrm{JPC}=3.6 \mathrm{~Hz}, o^{\prime}-\mathrm{CH}_{3}{ }^{\mathrm{Mes}, \mathrm{a}}\right), 22.9\left(\mathrm{dd}, J=10.1 \mathrm{~Hz}, J=4.0 \mathrm{~Hz}, o^{-}\right.$ 
$\left.\mathrm{CH}_{3}{ }^{\mathrm{Mes}, \mathrm{a}}\right), 21.0\left(\mathrm{~m}, o_{-}-\mathrm{CH}_{3}{ }^{\text {Mes,b}}\right), 20.7\left(\mathrm{~m}, o^{\prime}-\mathrm{CH}_{3}{ }^{\mathrm{Mes}, \mathrm{b}}\right), 20.5\left(p-\mathrm{CH}_{3}{ }^{\mathrm{Mes}, \mathrm{a}}\right), 20.0\left(p-\mathrm{CH}_{3}{ }^{\mathrm{Mes}, \mathrm{b}}\right)$. [ $\mathrm{C}_{6} \mathrm{~F}_{5}$ not listed; ${ }^{\mathrm{t}}$ tentatively assigned].

${ }^{31} \mathrm{P}\left\{{ }^{1} \mathrm{H}\right\}$ NMR $\left(202 \mathrm{MHz}\right.$, dichloromethane- $\left.d_{2}, 193 \mathrm{~K}\right): \delta 4.1\left(v_{1 / 2} \sim 40 \mathrm{~Hz}\right)$.

${ }^{11} \mathrm{~B}\left\{{ }^{1} \mathrm{H}\right\}$ NMR (160 MHz, dichloromethane- $\left.d_{2}, 193 \mathrm{~K}\right): \delta 2.0$ (broad).

${ }^{19} \mathrm{~F}$ NMR $\left(470 \mathrm{MHz}\right.$, dichloromethane- $\left.d_{2}, 193 \mathrm{~K}\right): \delta-126.2(\mathrm{~m}, \mathrm{o}),-130.7\left(\mathrm{~m}, \mathrm{o}^{\prime}\right),-158.7$ (br t, $\left.{ }^{3} J_{\mathrm{FF}}=21.4 \mathrm{~Hz}, p\right),-164.3(\mathrm{~m}, \mathrm{~m}),-164.5\left(\mathrm{~m}, \mathrm{~m}^{\prime}\right)\left(\right.$ each $\left.1 \mathrm{~F}, \mathrm{C}_{6} \mathrm{~F}_{5}\right)\left[\Delta \delta^{19} \mathrm{~F}_{\mathrm{mp}}=5.6,5.8\right],-127.6(\mathrm{~m}$, $1 \mathrm{~F}, o),-129.1\left(\mathrm{~m}, 1 \mathrm{~F}, o^{\prime}\right),-157.8\left(\mathrm{brt}, 1 \mathrm{~F},{ }^{3} \mathrm{JFF}_{\mathrm{FF}}=21.6 \mathrm{~Hz}, p\right),-165.5\left(\mathrm{~m}, 2 \mathrm{~F}, m, m^{\prime}\right)\left(\mathrm{C}_{6} \mathrm{~F}_{5}\right)\left[\Delta \delta^{19} \mathrm{~F}_{\mathrm{mp}}\right.$ $=7.7]$.
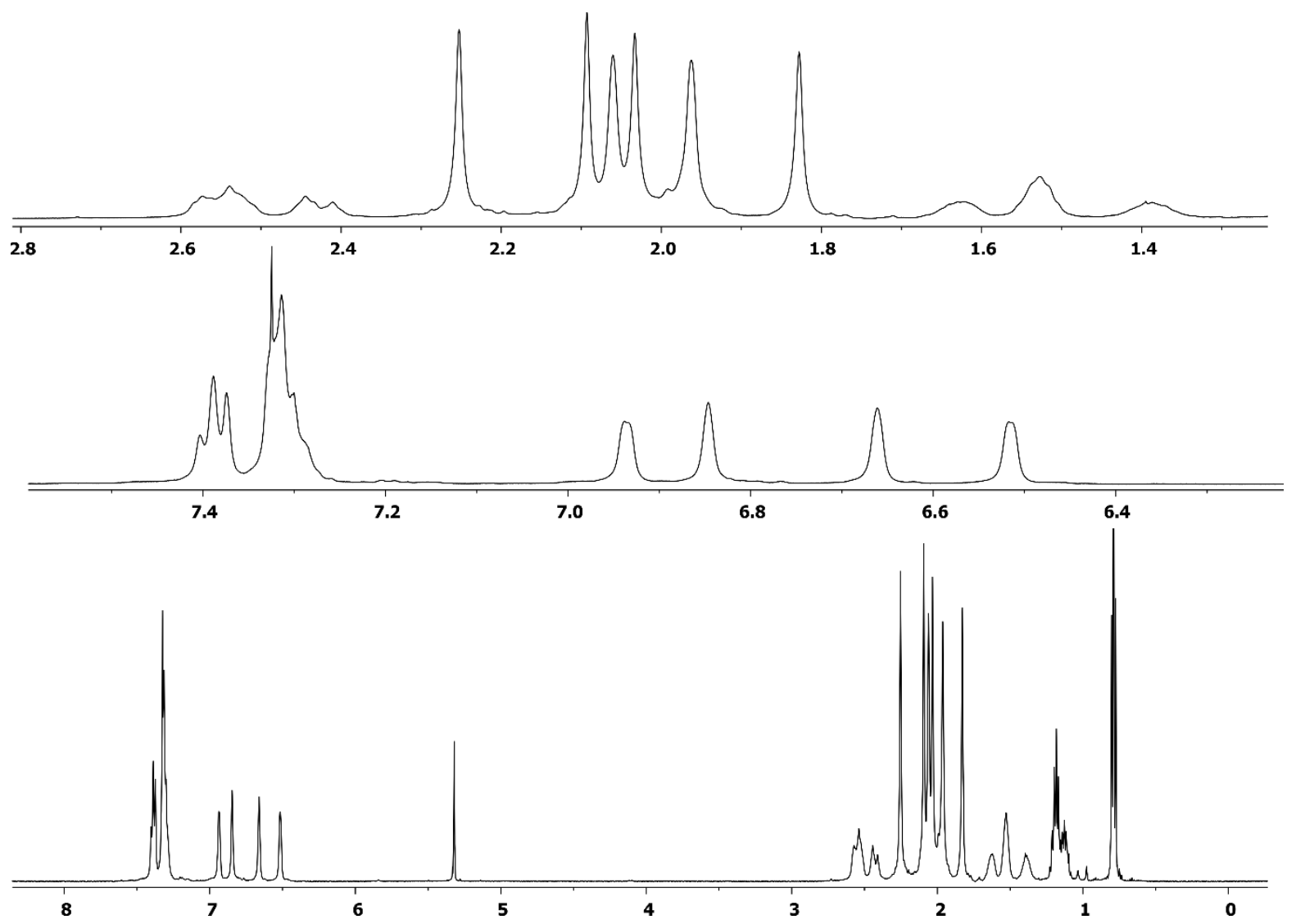

Figure S27: ${ }^{1} \mathbf{H}$ NMR (500 MHz, dichloromethane- $\left.d_{2}, 193 \mathrm{~K}\right)$ spectrum of compound 12 [admixed with pentane] 

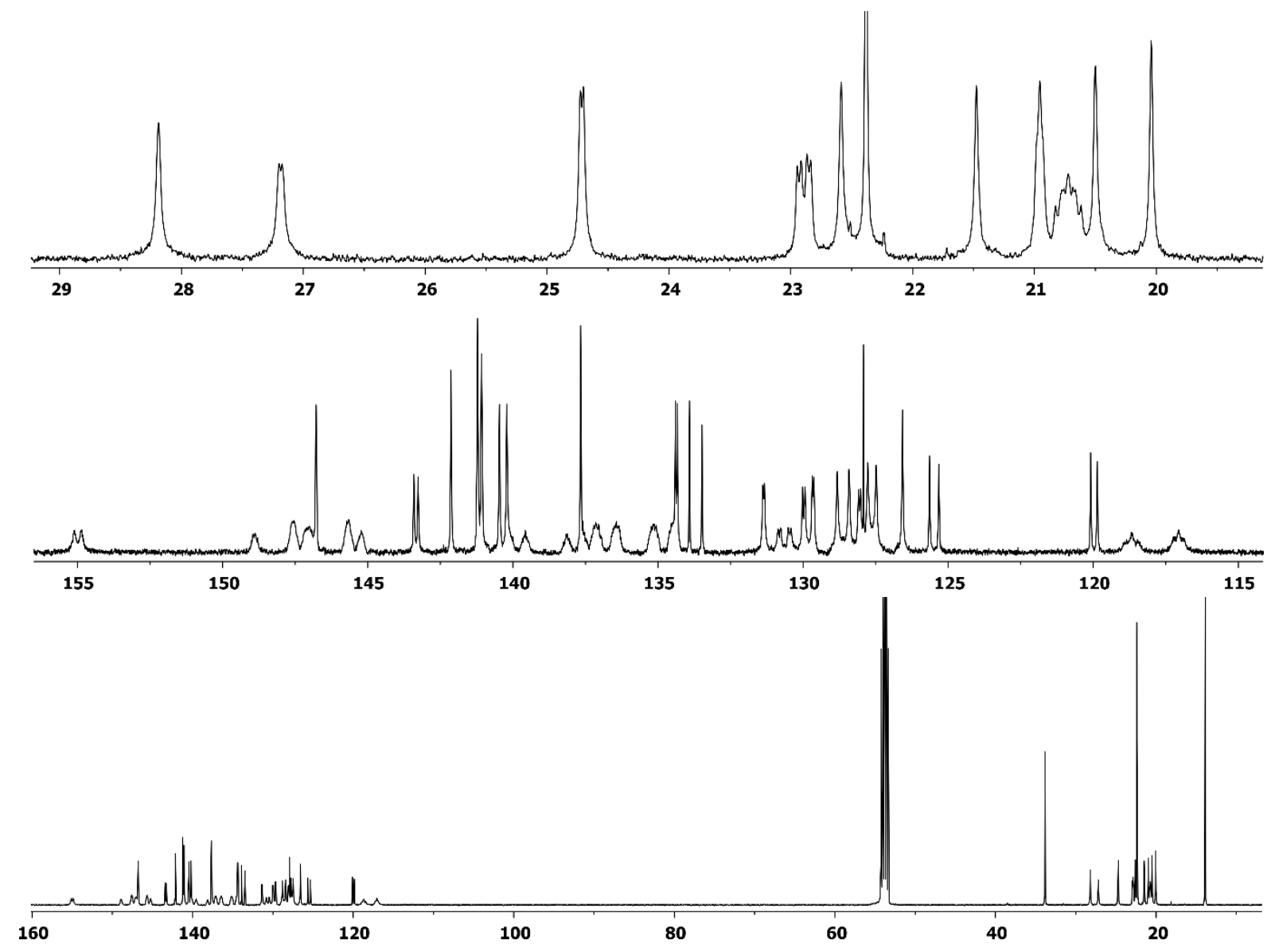

Figure S28: ${ }^{13} \mathbf{C}\left\{{ }^{1} \mathrm{H}\right\}$ NMR $(126 \mathrm{MHz}$, dichloromethane-d, $193 \mathrm{~K})$ spectrum of compound 12 [admixed with pentane]

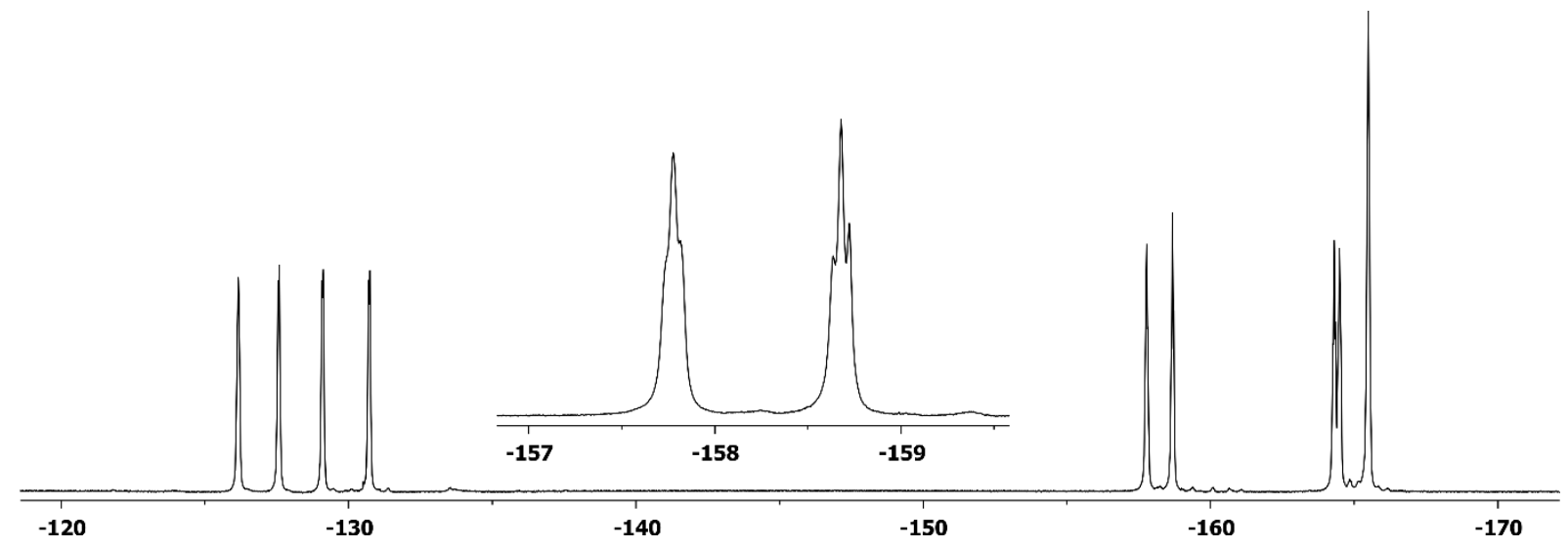

Figure S29: ${ }^{19} \mathrm{~F}$ NMR (470 MHz, dichloromethane- $d_{2}, 193 \mathrm{~K}$ ) spectrum of compound 12 


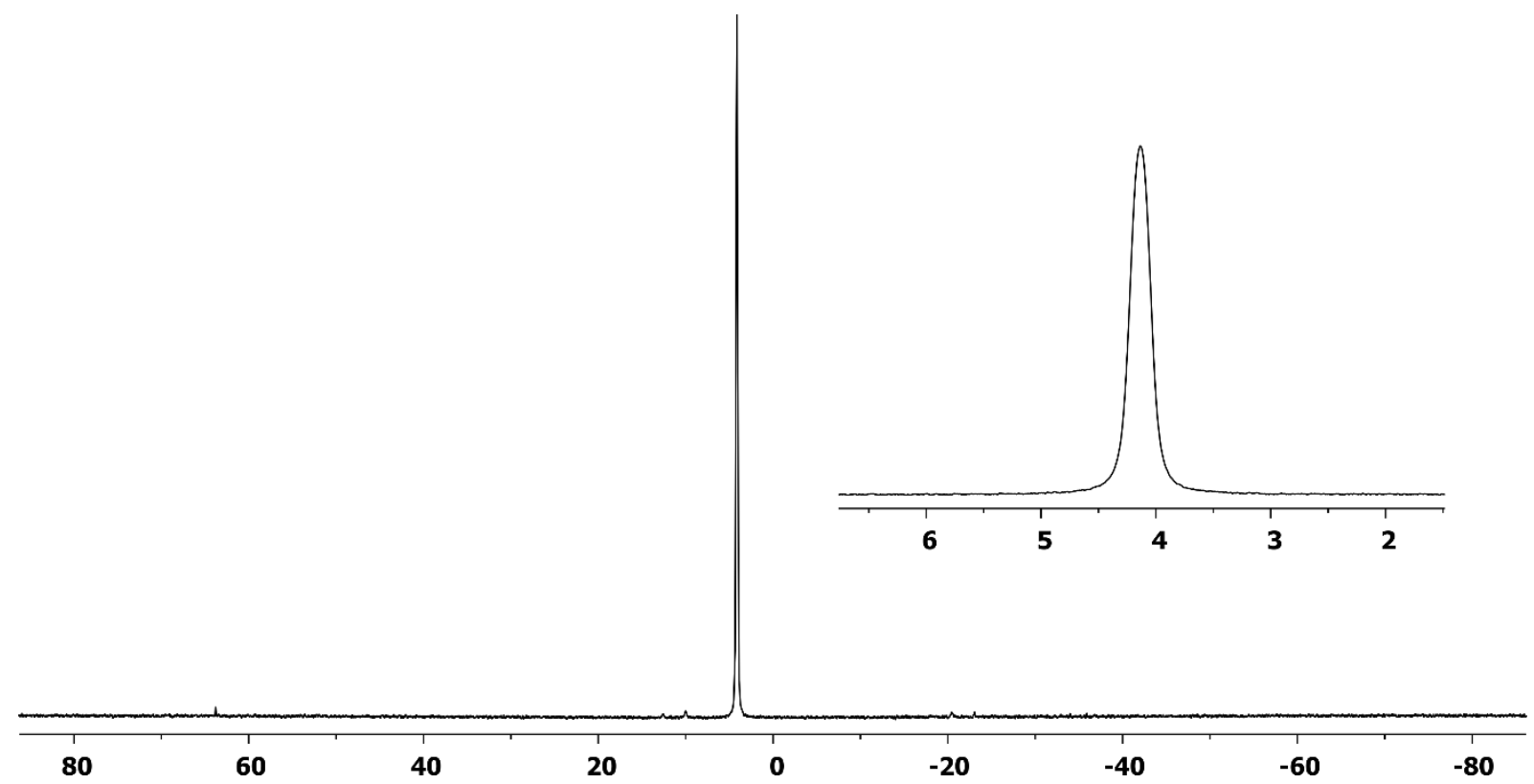

Figure S30: ${ }^{31} \mathbf{P}\left\{{ }^{1} \mathbf{H}\right\}$ NMR $\left(202 \mathrm{MHz}\right.$, dichloromethane- $\left.d_{2}, 193 \mathrm{~K}\right)$ spectrum of compound 12

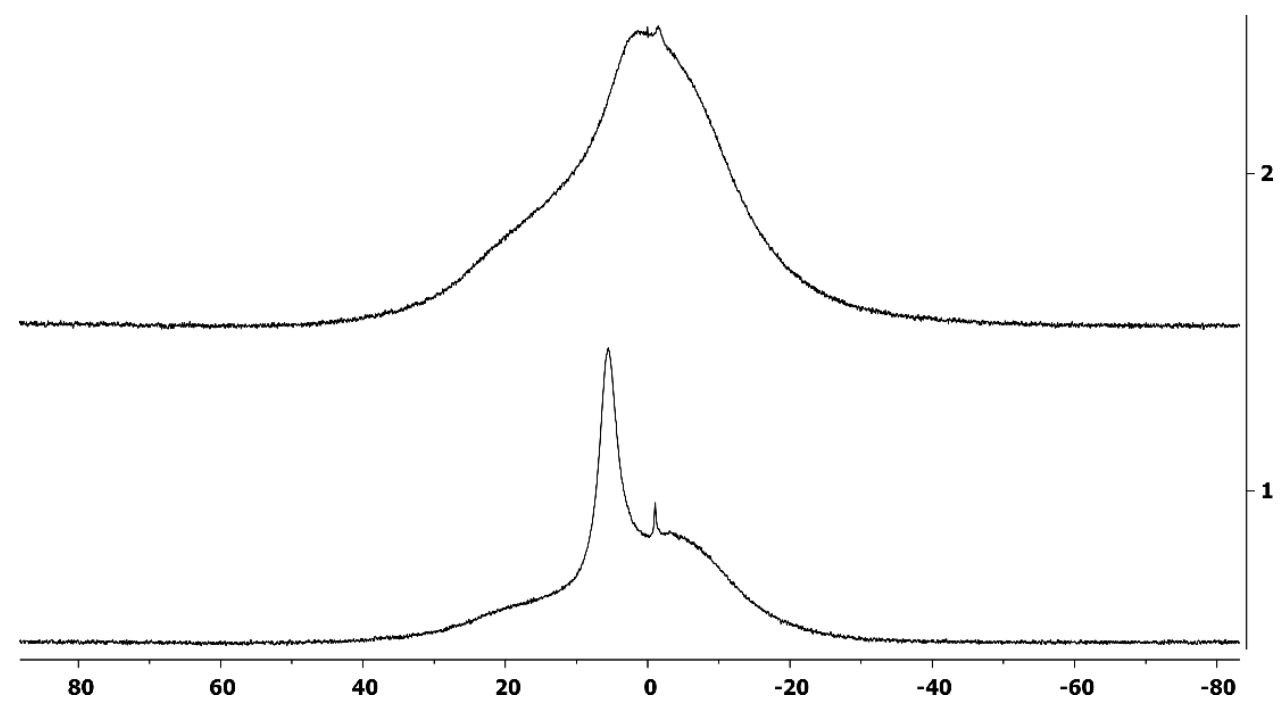

Figure S31: ${ }^{11} \mathrm{~B}\left\{{ }^{1} \mathrm{H}\right\}$ NMR (160 MHz, dichloromethane- $\left.d_{2}\right)$ spectra of compound 12 at $299 \mathrm{~K}(1)$ and $193 \mathrm{~K}(2)$ 


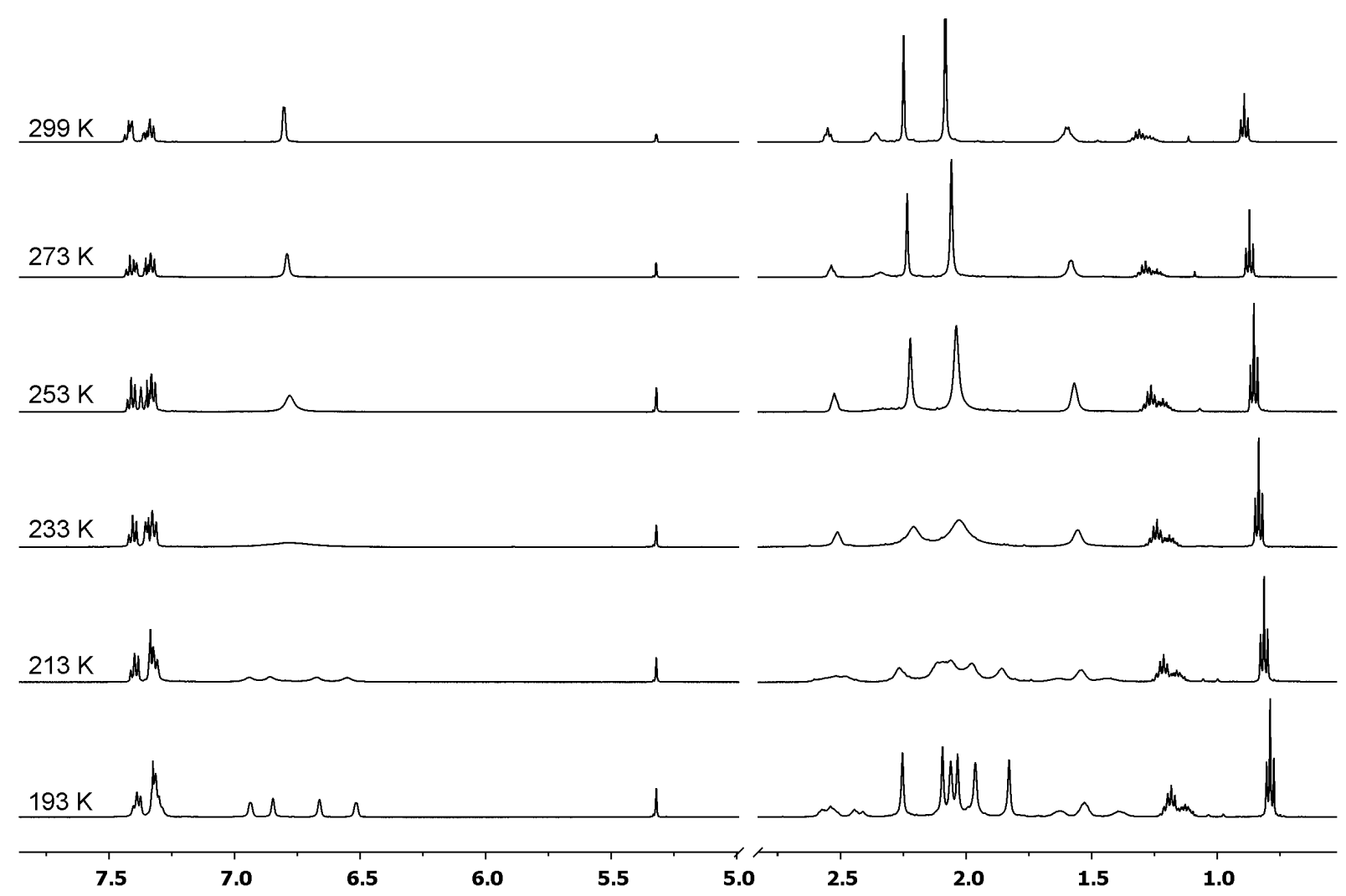

Figure S32: Dynamic ${ }^{1} \mathrm{H}$ NMR (500 MHz, dichloromethane- $\left.d_{2}\right)$ spectra of compound 12 [admixed with pentane]

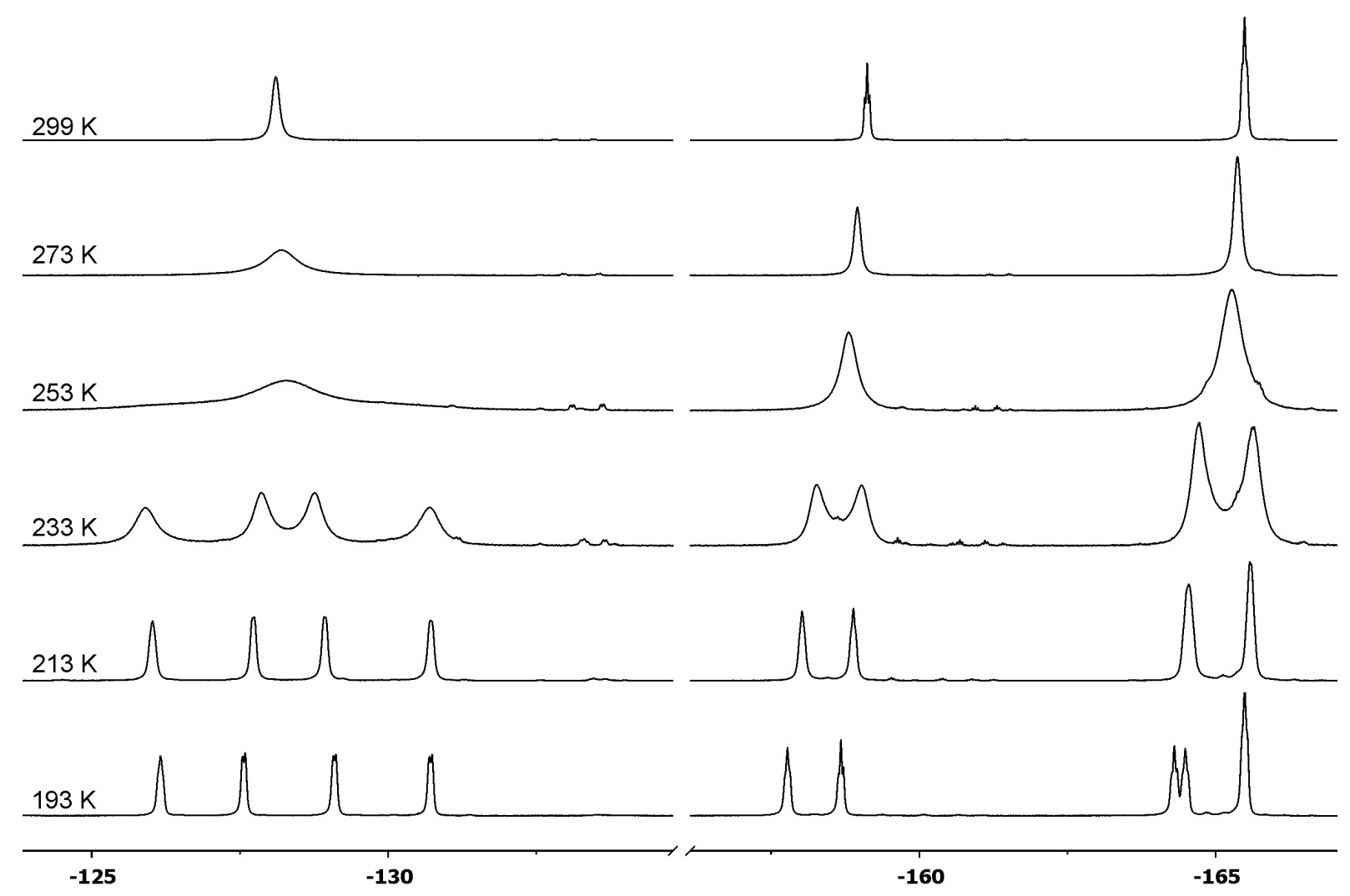

Figure S33: Dynamic ${ }^{19} \mathrm{~F} \mathrm{NMR}\left(470 \mathrm{MHz}\right.$, dichloromethane- $\left.d_{2}\right)$ spectra of compound 12 
$\Delta \mathrm{G}^{\ddagger}\left[\mathrm{T}_{\mathrm{c}}, \Delta v(\mathrm{~T})\right]=\mathrm{RT}_{\mathrm{c}}(22.96+\ln (\mathrm{Tc} / \Delta v))[\mathrm{J} / \mathrm{mol}]$

$\mathrm{T}_{\mathrm{c}}=$ coalescence temperature $[\mathrm{K}]: 243 \mathrm{~K}\left({ }^{19} \mathrm{~F}, p-\mathrm{BC}_{6} \mathrm{~F}_{5}\right)$

$\Delta v=$ chemical shift difference $[\mathrm{Hz}]\left({ }^{19} \mathrm{~F}, p-\mathrm{BC}_{6} \mathrm{~F}_{5}, 193 \mathrm{~K}\right): 426 \mathrm{~Hz}$

$\mathrm{R}=8.314 \mathrm{~J} /(\mathrm{mol} \cdot \mathrm{K}) ; 1 \mathrm{~J}=0.239 \mathrm{cal}$

$\Delta \mathrm{G} \mp[243 \mathrm{~K}, \Delta v(193 \mathrm{~K})=426 \mathrm{~Hz}]=45.251 \mathrm{~J} / \mathrm{mol}=10.8 \pm 0.3 \mathrm{kcal} / \mathrm{mol}$

Crystals suitable for the X-ray crystal structure analysis were obtained by slow diffusion of pentane into a solution of compound 12 in dichloromethane at $-35^{\circ} \mathrm{C}$ :

X-ray crystal structure analysis of compound 12: formula $\mathrm{C}_{46} \mathrm{H}_{36} \mathrm{BF}_{10} \mathrm{P}, M=820.53$, colourless crystal, $0.08 \times 0.06 \times 0.02 \mathrm{~mm}, a=13.8302(3), b=23.4539(5), c=14.8556(3) \AA$ $=108.613(1)^{\circ}, V=4566.7(2) \AA^{3}, \rho_{\text {calc }}=1.193 \mathrm{gcm}^{-3}, \mu=0.130 \mathrm{~mm}^{-1}$, empirical absorption correction (0.989 $\leq \mathrm{T} \leq 0.997), Z=4$, monoclinic, space group $P 2_{1} / \mathrm{n}$ (No. 14$), \lambda=0.71073 \AA$, $T=223(2) \mathrm{K}, \omega$ and $\varphi$ scans, 27050 reflections collected $( \pm h, \pm k, \pm 1), 7819$ independent $\left(R_{\text {int }}=\right.$ $0.103)$ and 5035 observed reflections $[>2 \sigma(I)], 529$ refined parameters, $R=0.106, w R^{2}=$ 0.234 , max. (min.) residual electron density $0.90(-0.47)$ e. $\AA^{-3}$, hydrogen atoms were calculated and refined as riding atoms.

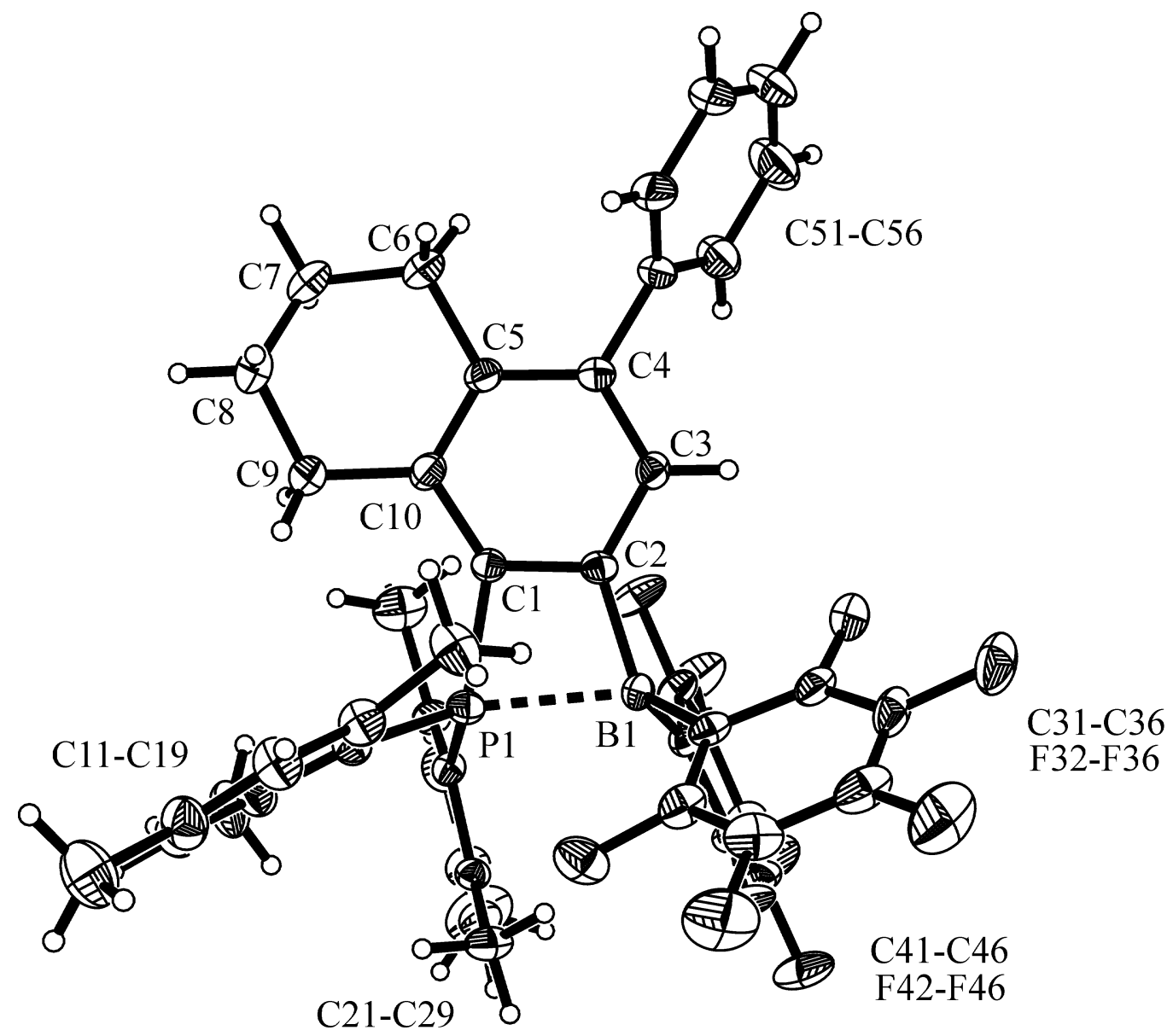

Figure S34: X-ray crystal structure of compound 12 (thermal ellipsoids are shown at the 30\% probability level) 


\section{Synthesis of compound 13}

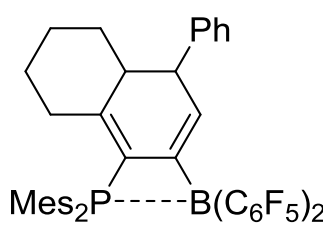

11

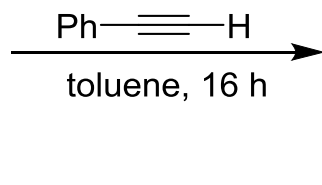

Scheme S6

Phenylacetylene $(12.4 \mathrm{mg}, 122 \mu \mathrm{mol}, 1.0 \mathrm{eq})$ was added dropwise to a solution of compound 11 (100 mg, $122 \mu \mathrm{mol}, 1.0 \mathrm{eq})$ in toluene $(4 \mathrm{~mL})$ at ambient temperature and then the reaction mixture was stirred for 16 hours at $80^{\circ} \mathrm{C}$. Subsequently all volatiles were removed in vacuo and the resulting yellow oil suspended in pentane $(2 \mathrm{~mL})$. Then the reaction suspension was dried in vacuo and the resulting solid was washed with pentane $(3 \times 2 \mathrm{~mL})$. After drying in vacuo, compound 13 was obtained as a white solid (43.8 mg, 47.4 mol; 39\%).

IR $(\mathrm{KBr}): \tilde{v}\left[\mathrm{~cm}^{-1}\right]=3060(\mathrm{w}), 3027(\mathrm{w}), 2931$ (w), 2361 (w) $1945(\mathrm{w}), 1747(\mathrm{w}), 1639(\mathrm{w}), 1603$ $(w), 1547(w), 1511(m), 1452(s), 1382(w), 1296(w), 1269(m), 1248(m), 1154(w), 1083(s)$, 1033 (w), 966 (s), $910(w), 854(w), 784(w), 757(m), 738(w), 696(m), 645(m), 605(w), 558$ $(w), 490(w), 421(w)$.

M.p. $182^{\circ} \mathrm{C}$.

Anal. Calc. for $\mathrm{C}_{54} \mathrm{H}_{44} \mathrm{BF} 10 \mathrm{P}: \mathrm{C}: 70.14 ; \mathrm{H}: 4.80$. Found: C: $68.60 ; \mathrm{H}: 4.56$.

${ }^{1} \mathrm{H}$ NMR $\left(500 \mathrm{MHz}\right.$, dichloromethane- $\left.d_{2}, 253 \mathrm{~K}\right): \delta 9.70\left(\mathrm{~d},{ }^{1} \mathrm{~J}_{\mathrm{PH}}=495.9 \mathrm{~Hz}, \mathrm{PH}\right), 7.31(\mathrm{~m}, 2 \mathrm{H}$,

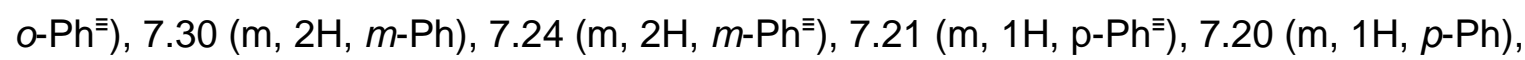
$7.15(\mathrm{~m}, 2 \mathrm{H}, 0-\mathrm{Ph}), 6.91\left(\mathrm{br} \mathrm{d},{ }^{3} \mathrm{~J}_{\mathrm{PH}}=4.1 \mathrm{~Hz}, 1 \mathrm{H}, m-\mathrm{Mes}^{\mathrm{a}}\right), 6.84\left(\mathrm{br} \mathrm{d},{ }^{3} \mathrm{JPH}_{\mathrm{PH}}=4.4 \mathrm{~Hz}, 1 \mathrm{H}, m-\right.$ $\mathrm{Mes}^{\mathrm{b}}$ ), $6.82\left(\mathrm{br} \mathrm{d},{ }^{3} \mathrm{JPH}_{\mathrm{PH}}=3.8 \mathrm{~Hz}, 1 \mathrm{H}, \mathrm{m}^{\prime}-\mathrm{Mes}^{\mathrm{a}}\right), 6.77\left(\mathrm{br} \mathrm{d},{ }^{3} J_{\mathrm{PH}}=4.6 \mathrm{~Hz}, 1 \mathrm{H}, m^{\prime}-\mathrm{Mes}^{\mathrm{b}}\right), 5.90$ (br, 1H, 3-CH), $3.82\left(\mathrm{dm},{ }^{3} \mathrm{~J}_{\mathrm{HH}}=8.8 \mathrm{~Hz}, 1 \mathrm{H}, 4-\mathrm{CH}\right), 2.66\left(\mathrm{~s}, 3 \mathrm{H}, 0-\mathrm{CH}_{3}{ }^{\mathrm{Mes}, \mathrm{b}}\right), 2.65(\mathrm{~s}, 3 \mathrm{H}, 0-$ $\mathrm{CH}_{3}{ }^{\text {Mes,a }}$ ), 2.52/2.04, 1.72/1.24, 1.63/1.17, 1.47/0.95 (each $\mathrm{m}$, each $\left.1 \mathrm{H}, \mathrm{CH}_{2}\right), 2.34(\mathrm{~m}, 1 \mathrm{H}, 5-$ $\mathrm{CH}$ ), 2.26 (s, 3H, p- $\mathrm{CH}_{3}{ }^{\mathrm{Mes}, \mathrm{b}}$ ), 2.23 (s, 3H, $\left.p-\mathrm{CH}_{3}{ }^{\mathrm{Mes}, \mathrm{a}}\right), 1.89$ (s, 3H, o'- $\left.\mathrm{CH}_{3}{ }^{\mathrm{Mes}, \mathrm{b}}\right), 1.77$ (s, 3H, o' $\left.^{\prime}-\mathrm{CH}_{3}{ }^{\mathrm{Mes}, \mathrm{a}}\right)$.

${ }^{13} \mathrm{C}\left\{{ }^{1} \mathrm{H}\right\}$ NMR $\left(126 \mathrm{MHz}\right.$, dichloromethane- $\left.d_{2}, 253 \mathrm{~K}\right): \delta 168.4\left(\mathrm{~d},{ }^{2} \mathrm{~J}_{\mathrm{PC}}=7.7 \mathrm{~Hz}, 10-\mathrm{C}\right){ }^{\mathrm{t}}, 144.9$ $\left(\mathrm{d},{ }^{2} J_{\mathrm{PC}}=9.2 \mathrm{~Hz}, o-\mathrm{Mes}^{\mathrm{b}}\right), 144.8\left(\mathrm{~d},{ }^{4} J_{\mathrm{PC}}=2.8 \mathrm{~Hz}, p-\mathrm{Mes}^{\mathrm{a}}\right), 144.2\left(\mathrm{~d},{ }^{4} J_{\mathrm{PC}}=2.8 \mathrm{~Hz}, p-\mathrm{Mes}^{\mathrm{b}}\right)$, $143.4\left(\mathrm{~d},{ }^{2} J_{\mathrm{PC}}=11.0 \mathrm{~Hz}, o-\mathrm{Mes}^{\mathrm{a}}\right), 142.8\left(\mathrm{~d},{ }^{2} \mathrm{~J}_{\mathrm{PC}}=9.4 \mathrm{~Hz}, o^{\prime}-\mathrm{Mes}^{\mathrm{a}}\right), 142.4(i-\mathrm{Ph}), 142.2(\mathrm{~d}$, $\left.{ }^{2} J_{\mathrm{PC}}=10.9 \mathrm{~Hz}, \mathrm{o}^{\prime}-\mathrm{Mes}^{\mathrm{b}}\right), 134.6(\mathrm{br} \mathrm{dm}, J=14.2 \mathrm{~Hz}, 3-\mathrm{CH}), 132.3\left(\mathrm{~d},{ }^{3} J_{\mathrm{PC}}=11.3 \mathrm{~Hz}, \mathrm{~m}^{\prime}-\right.$ $\left.\operatorname{Mes}^{\mathrm{a}}\right), 131.6\left(\mathrm{~d},{ }^{3} \mathrm{JPC}_{\mathrm{PC}}=11.5 \mathrm{~Hz}, m-\mathrm{Mes}^{\mathrm{b}}\right), 131.2\left(o-\mathrm{Ph}^{\equiv}\right), 130.6\left(\mathrm{~d},{ }^{3} J_{\mathrm{PC}}=10.8 \mathrm{~Hz}, m^{\prime}-\mathrm{Mes}^{\mathrm{b}}\right)$, $130.4\left(\mathrm{~d},{ }^{3} \mathrm{JPC}=10.7 \mathrm{~Hz}, m-\mathrm{Mes}^{\mathrm{a}}\right), 129.0(o-\mathrm{Ph}), 128.2(m-\mathrm{Ph}), 128.1\left(m-\mathrm{Ph}^{\equiv}\right), 126.9\left(i-\mathrm{Ph}^{\equiv}\right)$, $126.5(p-\mathrm{Ph} \equiv), 126.2(p-\mathrm{Ph}), 118.9\left(\mathrm{~d},{ }^{1} J_{\mathrm{PC}}=77.8 \mathrm{~Hz}, i-\mathrm{Mes}^{\mathrm{a}}\right), 115.5\left(\mathrm{~d},{ }^{1} J_{\mathrm{PC}}=83.4 \mathrm{~Hz}, i-\right.$ 
Mes $\left.^{b}\right), 113.1\left(\mathrm{~d},{ }^{1} \mathrm{JPC}_{\mathrm{PC}}=73.1 \mathrm{~Hz}, 1-\mathrm{C}\right) \mathrm{t}, 110.3\left(\mathrm{br} 1: 1: 1: 1 \mathrm{q},{ }^{1} \mathrm{~J}_{\mathrm{CB}} \sim 80 \mathrm{~Hz}, \mathrm{BC} \equiv\right), 98.7$ (br,

$\mathrm{PhC} \equiv), 51.3\left(\mathrm{~d},{ }^{3} \mathrm{JPC}_{\mathrm{PC}}=12.4 \mathrm{~Hz}, 5-\mathrm{CH}\right), 43.0(4-\mathrm{CH}), 35.0\left(\mathrm{~d},{ }^{3} \mathrm{~J}_{\mathrm{PC}}=9.8 \mathrm{~Hz}\right), 31.7,29.2,26.3$ $\left(\mathrm{CH}_{2}\right), 23.8\left(\mathrm{~d},{ }^{3} \mathrm{JPC}_{\mathrm{PC}}=10.7 \mathrm{~Hz}, o-\mathrm{CH}_{3}{ }^{\mathrm{Mes}, \mathrm{a}}\right), 22.3\left(\mathrm{~d},{ }^{3} \mathrm{JPC}_{\mathrm{PC}}=5.8 \mathrm{~Hz}, \mathrm{o}^{\prime}-\mathrm{CH}_{3}{ }^{\mathrm{Mes}, \mathrm{a}}\right), 21.5\left(\mathrm{~d},{ }^{3} \mathrm{~J}_{\mathrm{PC}}=\right.$ $\left.4.4 \mathrm{~Hz}, o-\mathrm{CH}_{3}{ }^{\mathrm{Mes}, \mathrm{b}}\right), 21.1\left(p-\mathrm{CH}_{3}{ }^{\mathrm{Mes}, \mathrm{a}}\right), 20.9\left(p-\mathrm{CH}_{3}{ }^{\mathrm{Mes}, \mathrm{b}}\right), 20.4\left(\mathrm{~d},{ }^{3} \mathrm{JPC}_{\mathrm{PC}}=10.4 \mathrm{~Hz}, o^{\prime}-\mathrm{CH}_{3}{ }^{\mathrm{Mes}, \mathrm{b}}\right)$, n.o. (2-C). [ $\mathrm{C}_{6} \mathrm{~F}_{5}$ not listed; ${ }^{\mathrm{t}}$ tentatively assigned].

${ }^{31} \mathbf{P}$ NMR (202 MHz, dichloromethane- $\left.d_{2}, 253 \mathrm{~K}\right): \delta-29.3\left(\mathrm{~d},{ }^{1} \mathrm{~J}_{\mathrm{PH}}=496.6 \mathrm{~Hz}\right)$.

${ }^{31} \mathbf{P}\left\{{ }^{1} \mathbf{H}\right\}$ NMR $\left(202 \mathrm{MHz}\right.$, dichloromethane- $\left.d_{2}, 253 \mathrm{~K}\right): \delta-29.3\left(v_{1 / 2} \sim 15 \mathrm{~Hz}\right)$.

${ }^{11} \mathbf{B}\left\{{ }^{1} \mathrm{H}\right\}$ NMR $\left(160 \mathrm{MHz}\right.$, dichloromethane- $\left.d_{2}, 253 \mathrm{~K}\right): \delta-17.7\left(v_{1 / 2} \sim 50 \mathrm{~Hz}\right)$

${ }^{19} \mathrm{~F}$ NMR $\left(470 \mathrm{MHz}\right.$, dichloromethane- $\left.d_{2}, 253 \mathrm{~K}\right): \delta-129.2(\mathrm{br}, 2 \mathrm{~F}, 0),-162.5\left(\mathrm{t},{ }^{3} \mathrm{JFF}_{\mathrm{FF}}=20.8 \mathrm{~Hz}\right.$, $1 \mathrm{~F}, p),-166.5(\mathrm{~m}, 2 \mathrm{~F}, m)\left(\mathrm{C}_{6} \mathrm{~F}_{5}\right)\left[\Delta \delta^{19} \mathrm{~F}_{\mathrm{mp}}=3.2\right],-129.6(\mathrm{~m}, 2 \mathrm{~F}, o),-161.0\left(\mathrm{t},{ }^{3} J_{\mathrm{FF}}=20.7 \mathrm{~Hz}, 1 \mathrm{~F}\right.$, p), $-165.9(\mathrm{~m}, 2 \mathrm{~F}, m)\left(\mathrm{C}_{6} \mathrm{~F}_{5}\right)\left[\Delta \delta^{19} \mathrm{~F}_{\mathrm{mp}}=4.9\right]$.
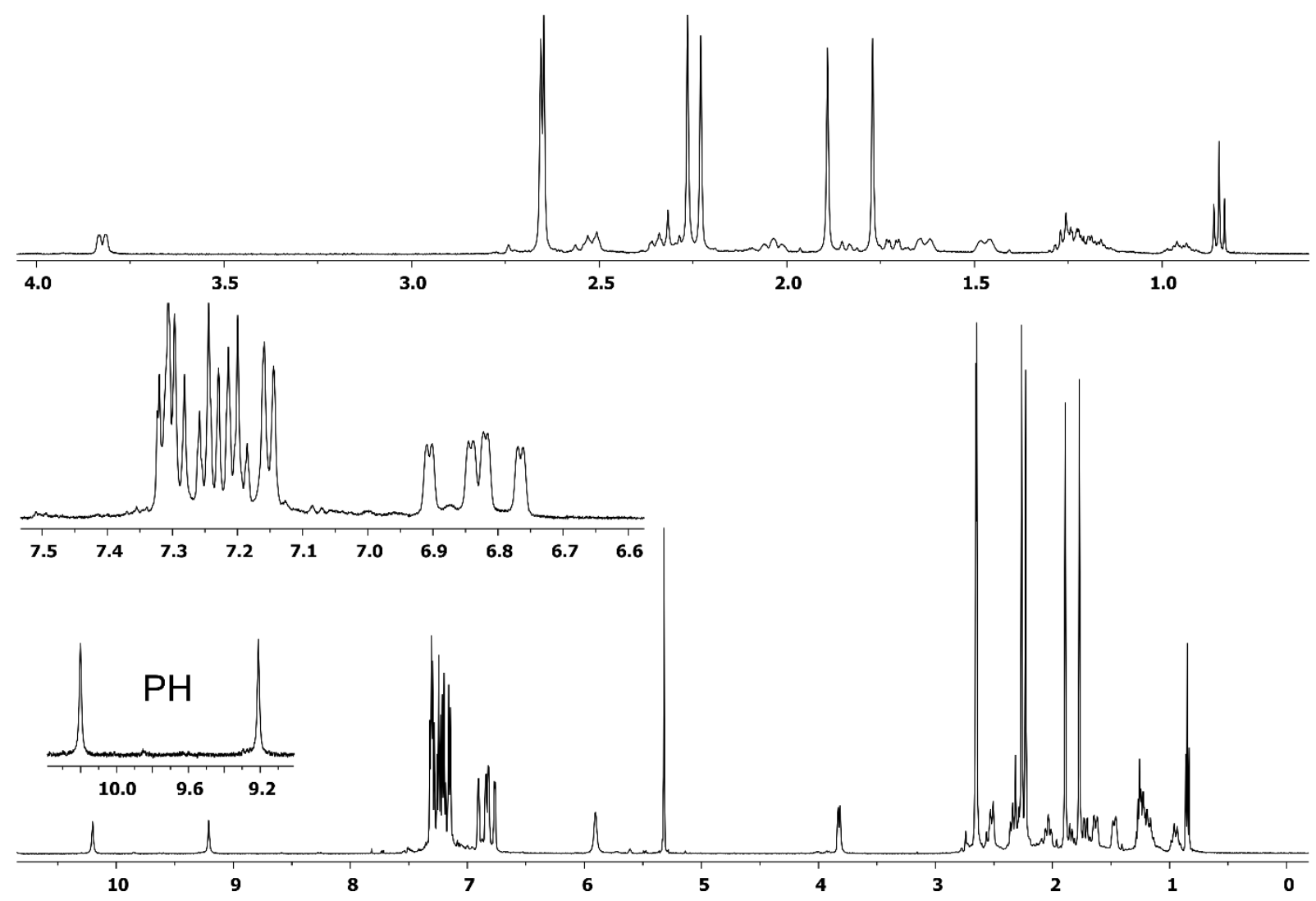

Figure S35: ${ }^{1} \mathbf{H}$ NMR $\left(500 \mathrm{MHz}\right.$, dichloromethane- $\left.d_{2}, 253 \mathrm{~K}\right)$ spectrum of compound 13 

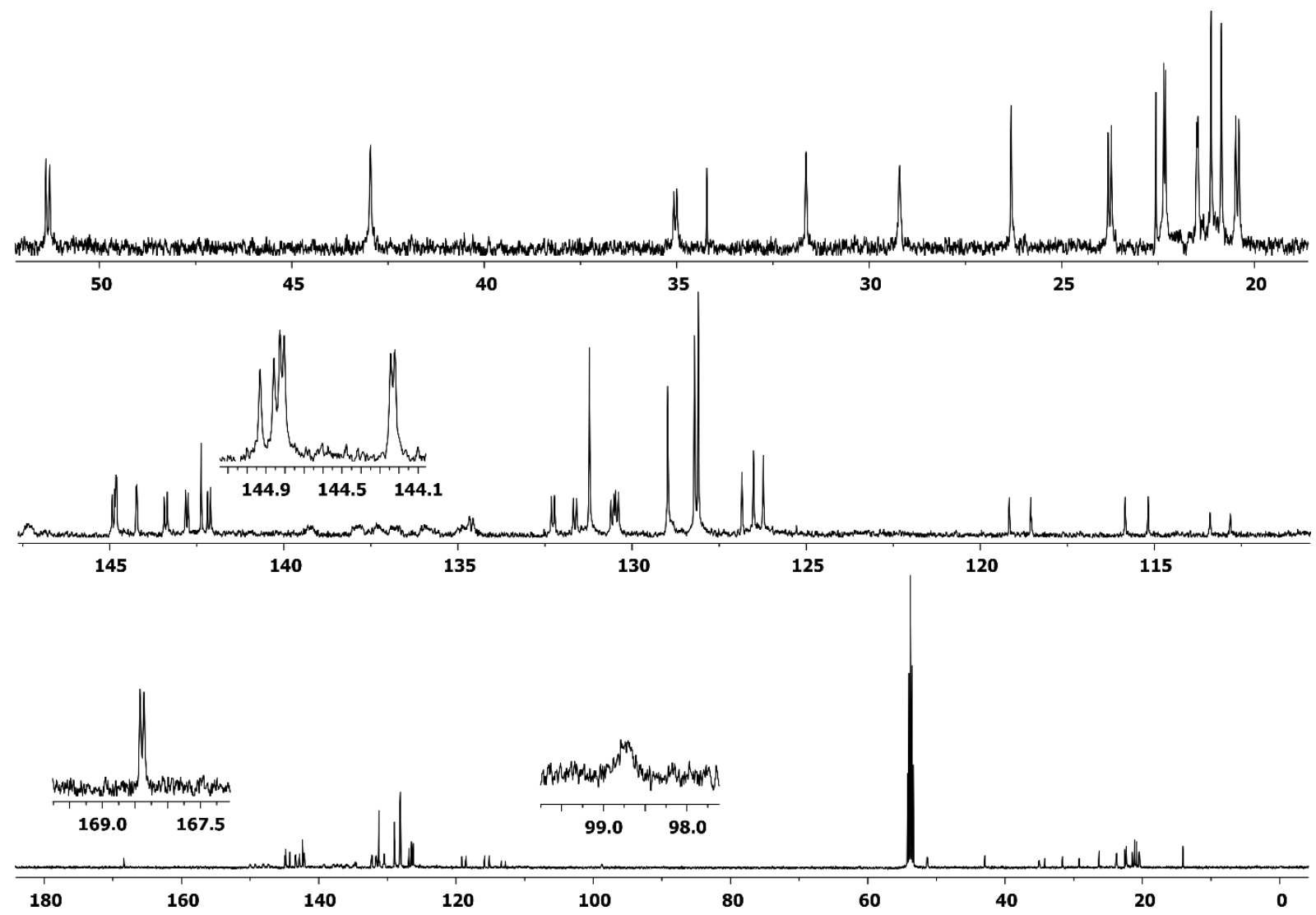

Figure S36: ${ }^{13} \mathrm{C}$ NMR (126 MHz, dichloromethane-d2, $253 \mathrm{~K}$ ) spectrum of compound 13

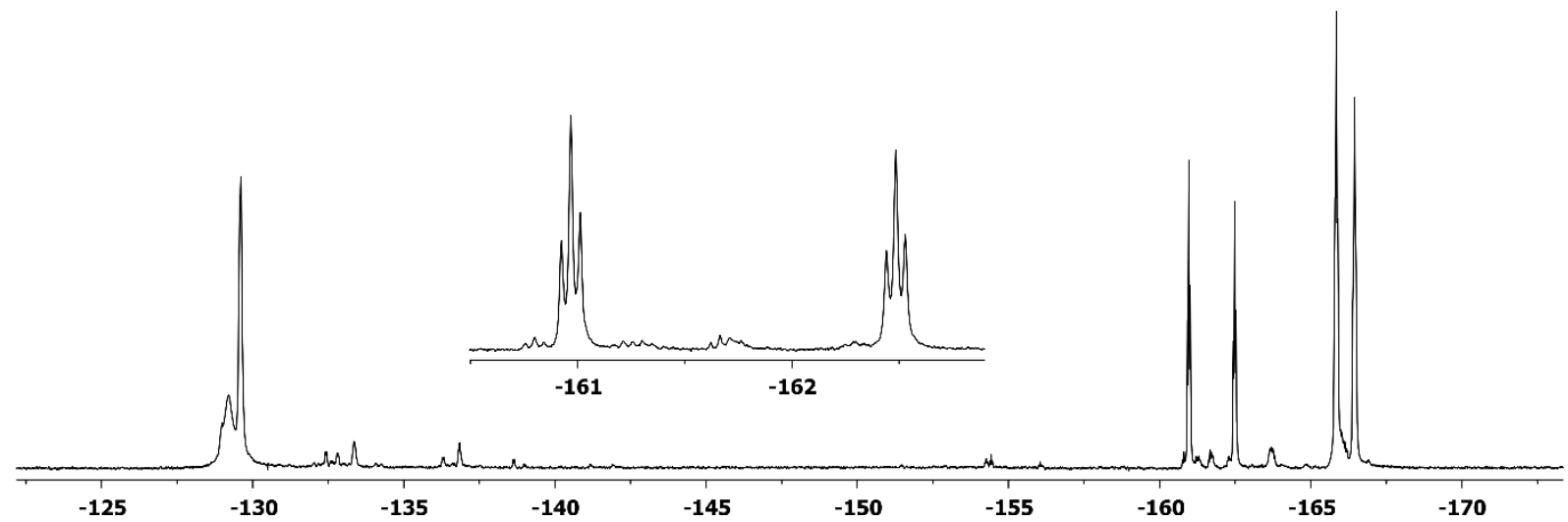

Figure S37: ${ }^{19} \mathrm{~F}$ NMR $\left(470 \mathrm{MHz}\right.$, dichloromethane- $\left.d_{2}, 253 \mathrm{~K}\right)$ spectrum of compound 13 


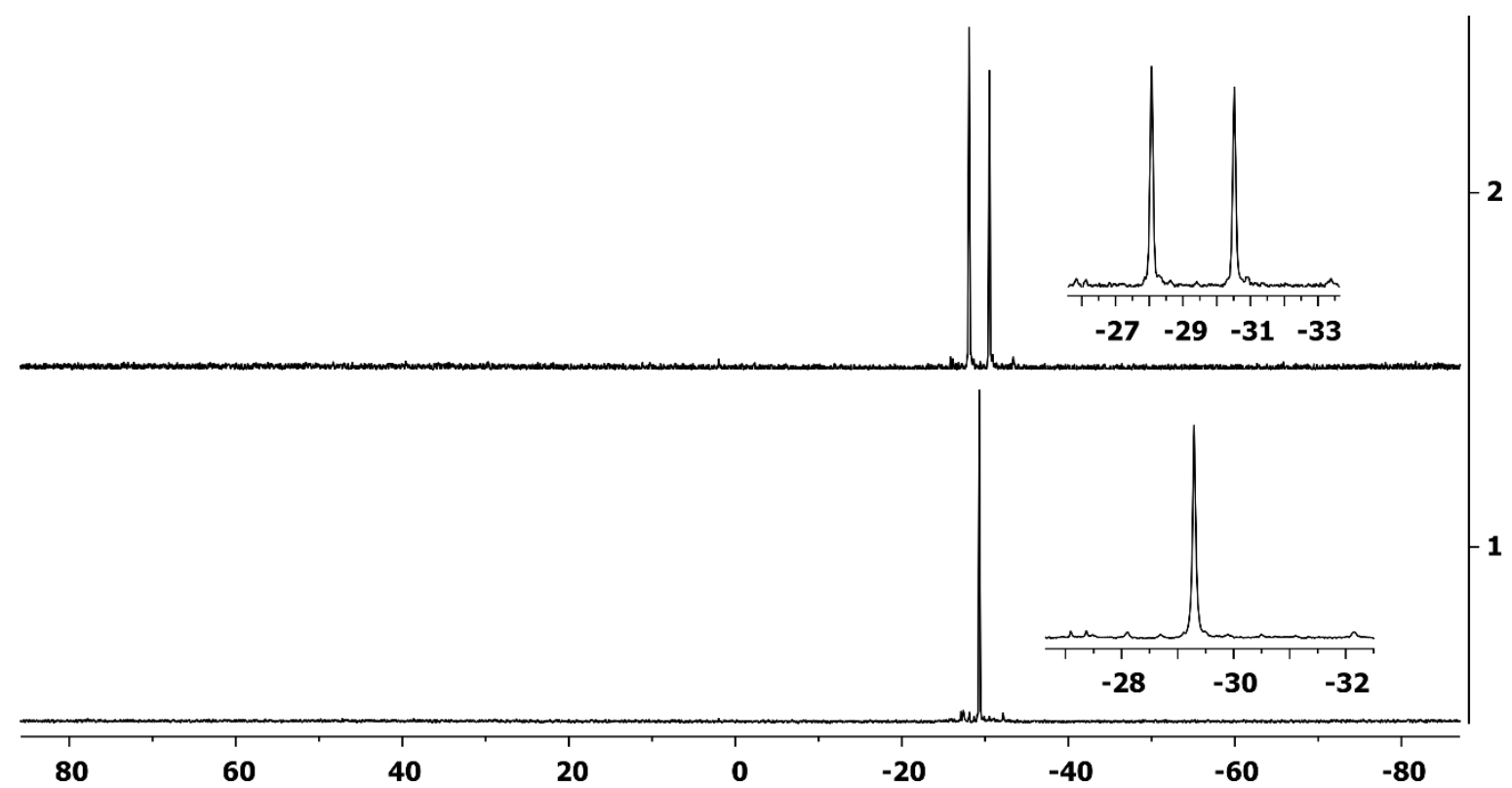

Figure S38: ${ }^{31} \mathbf{P}\left\{{ }^{1} \mathrm{H}\right\}$ NMR (1) and ${ }^{31} \mathbf{P}$ NMR (2) (202 MHz, dichloromethane- $d_{2}, 253 \mathrm{~K}$ ) spectra of compound 13

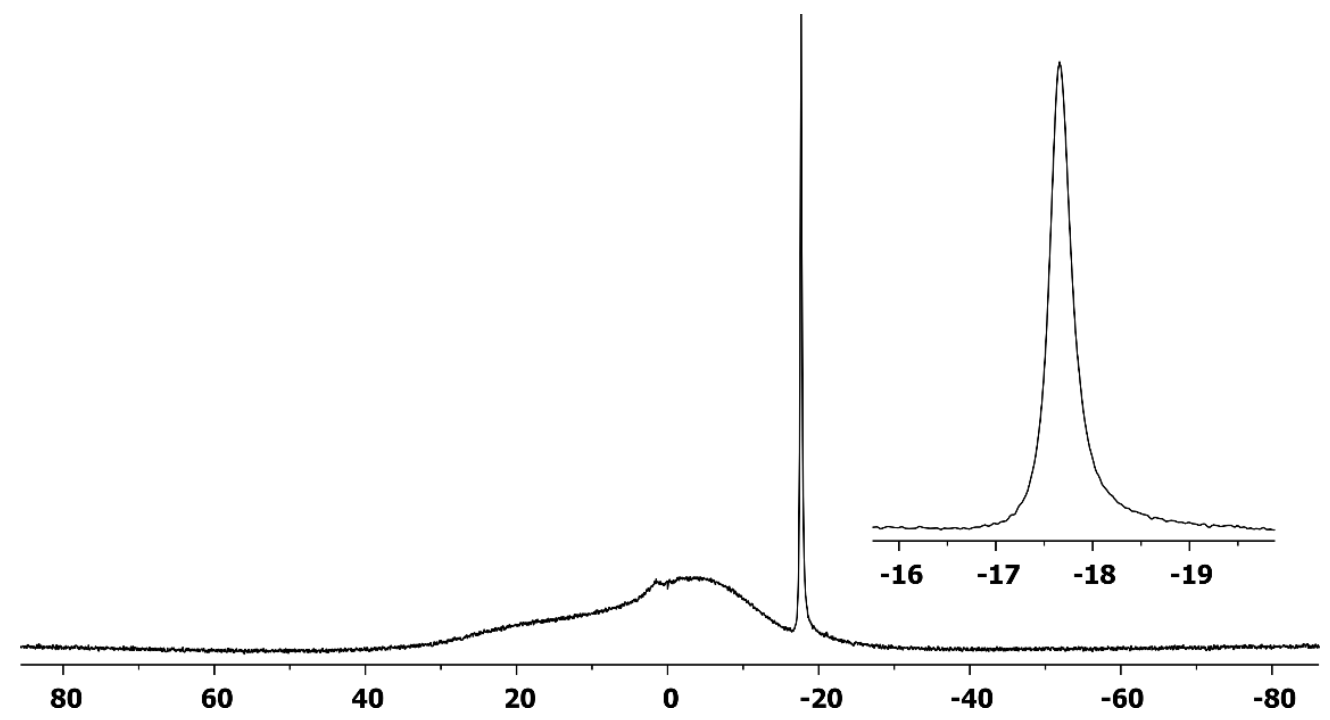

Figure S39: ${ }^{11} \mathrm{~B}\left\{{ }^{1} \mathrm{H}\right\}$ NMR $\left(160 \mathrm{MHz}\right.$, dichloromethane- $\left.d_{2}, 253 \mathrm{~K}\right)$ spectrum of compound 13

Crystals suitable for the X-ray crystal structure analysis were obtained from a dichloromethane solution of compound 13 at $-35^{\circ} \mathrm{C}$ :

X-ray crystal structure analysis of compound 13: A colorless prism-like specimen of $\mathrm{C}_{61} \mathrm{H}_{52} \mathrm{BF}_{10} \mathrm{P}$, approximate dimensions $0.180 \mathrm{~mm} \times 0.180 \mathrm{~mm} \times 0.200 \mathrm{~mm}$, was used for the X-ray crystallographic analysis. The X-ray intensity data were measured. A total of 460 frames were collected. The total exposure time was 4.47 hours. The frames were integrated with the Bruker SAINT software package using a narrow-frame algorithm. The integration of the data using a monoclinic unit cell yielded a total of 40631 reflections to a maximum $\theta$ angle of $26.40^{\circ}$ 
(0.80 $\AA$ resolution), of which 10234 were independent (average redundancy 3.970, completeness $\left.=99.9 \%, R_{\text {int }}=5.56 \%, R_{\text {sig }}=4.69 \%\right)$ and $8954(87.49 \%)$ were greater than $2 \sigma\left(\mathrm{F}^{2}\right)$. The final cell constants of $\underline{a}=14.2250(7) \AA \AA$ $105.716(2)^{\circ}$, volume $=2504.1(2) \AA^{3}$, are based upon the refinement of the $X Y Z$-centroids of 9863 reflections above $20 \sigma(\mathrm{I})$ with $4.573^{\circ}<2 \theta<52.62^{\circ}$. Data were corrected for absorption effects using the multi-scan method (SADABS). The ratio of minimum to maximum apparent transmission was 0.923 . The calculated minimum and maximum transmission coefficients (based on crystal size) are 0.9740 and 0.9760 . The structure was solved and refined using the Bruker SHELXTL Software Package, using the space group $P 1 \mathrm{n} 1$, with $Z=2$ for the formula unit, $\mathrm{C}_{61} \mathrm{H}_{52} \mathrm{BF} \mathrm{F}_{10} \mathrm{P}$. The final anisotropic full-matrix least-squares refinement on $\mathrm{F}^{2}$ with 669 variables converged at $\mathrm{R} 1=3.89 \%$, for the observed data and $\mathrm{wR} 2=8.45 \%$ for all data. The goodness-of-fit was 1.042 . The largest peak in the final difference electron density synthesis was $0.194 \mathrm{e}^{-} / \AA^{3}$ and the largest hole was $-0.226 \mathrm{e}^{-} / \AA^{3}$ with an RMS deviation of $0.045 \mathrm{e}^{-} / \AA^{3}$. On the basis of the final model, the calculated density was $1.349 \mathrm{~g} / \mathrm{cm}^{3}$ and $F(000), 1056 \mathrm{e}$.

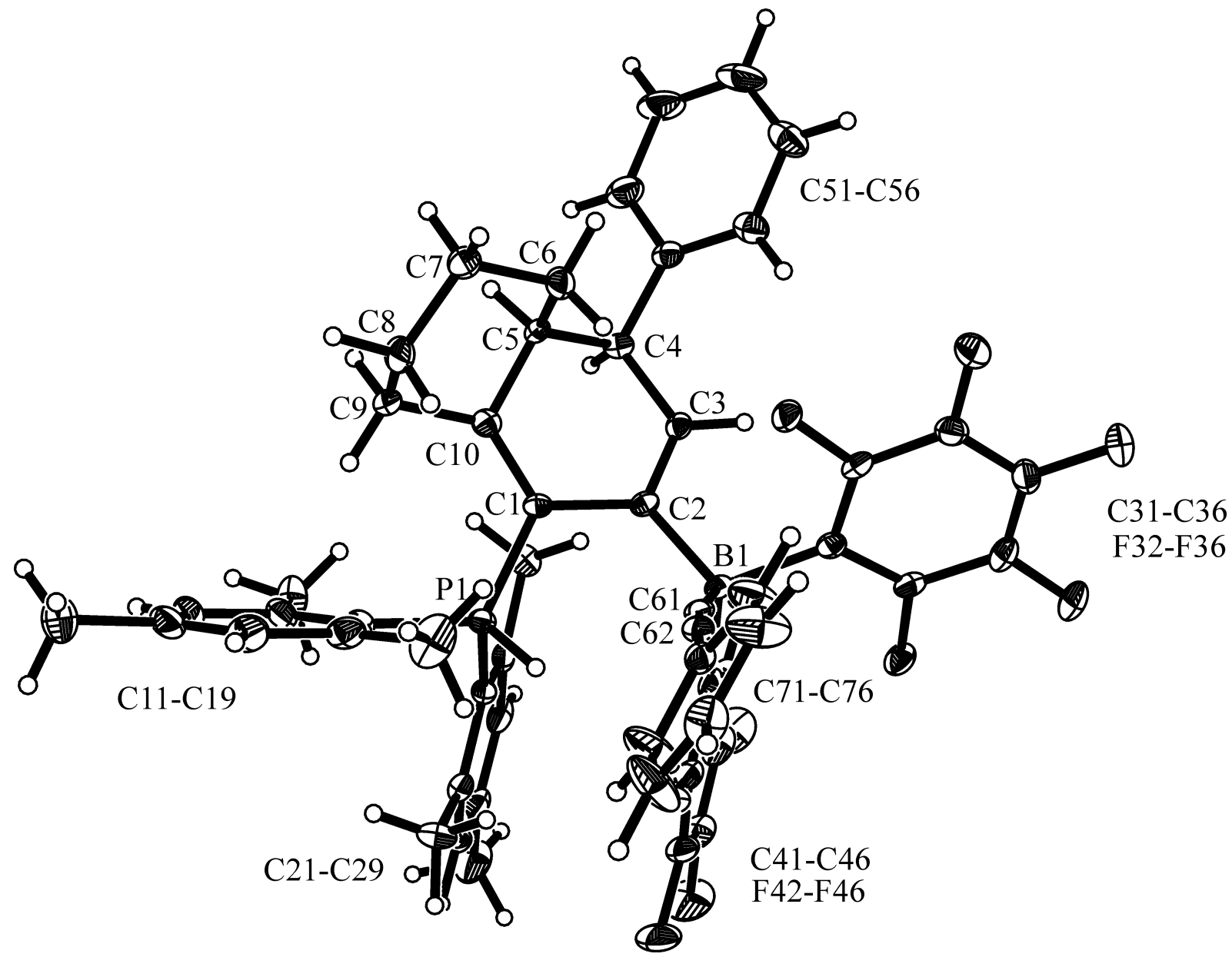

Figure S40: X-ray crystal structure of compound 13 (thermal ellipsoids are shown at the 50\% probability level) 


\section{Synthesis of compound 14}

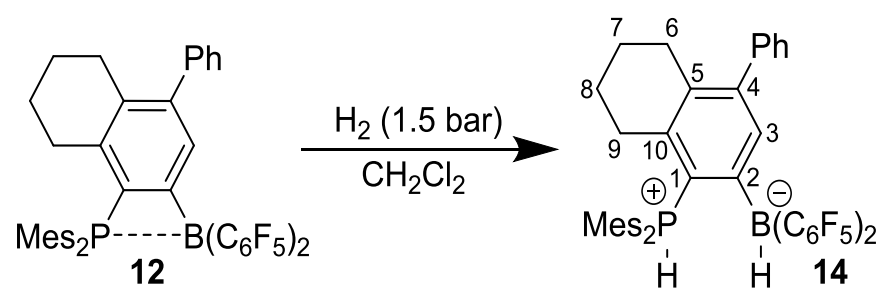

Scheme S7

A yellow solution of compound $12(200.0 \mathrm{mg}, 243.7 \mu \mathrm{mol}, 1.0 \mathrm{eq})$ in $\mathrm{CH}_{2} \mathrm{Cl}_{2}(2 \mathrm{~mL})$ was degassed at $-78^{\circ} \mathrm{C}$ and then exposed to a dihydrogen atmosphere (1.5 bar) at room temperature. The solution was stirred for $24 \mathrm{~h}$ at ambient temperature, before all volatiles were removed in vacuo. The obtained off-white residue was washed with pentane $(3 \times 2 \mathrm{~mL})$ and dried in vacuo to give compound 14 as a white solid (175.7 mg, $213.6 \mu \mathrm{mol}, 88 \%$ ).

IR $(\mathrm{KBr}): \tilde{v}\left[\mathrm{~cm}^{-1}\right]=3418(\mathrm{w}), 3316(\mathrm{w}), 3055(\mathrm{w}), 3028(\mathrm{w}), 2941(\mathrm{w}), 2854(\mathrm{w}), 2443(\mathrm{w}), 2360$ (w), $1939(w), 1775(w), 1639(m), 1604(m), 1559(w), 1509(s), 1461(s), 1380(m), 1332(w)$, $1293(w), 1271(\mathrm{~m}), 1206$ (w), 1176 (w), $1134(\mathrm{w}), 1083(\mathrm{~s}), 1030(\mathrm{~m}), 967$ (s), $905(\mathrm{~m}), 857$ $(\mathrm{m}), 768(\mathrm{~m}), 741(\mathrm{w}), 698(\mathrm{~m}), 678(\mathrm{w}), 647(\mathrm{~m}), 617(\mathrm{w}), 575(\mathrm{w}), 555(\mathrm{~m}), 512(\mathrm{w}), 488(\mathrm{w})$, $429(\mathrm{~m})$.

Decomp. $167^{\circ} \mathrm{C}$.

Anal. Calc. for $\mathrm{C}_{46} \mathrm{H}_{38} \mathrm{BF}{ }_{10} \mathrm{P}: \mathrm{C}: 67.17 ; \mathrm{H}:$ 4.66. Found: $\mathrm{C}: 67.36 ; \mathrm{H}: 5.28$.

${ }^{1} \mathrm{H}$ NMR $\left(600 \mathrm{MHz}\right.$, dichloromethane- $\left.d_{2}, 299 \mathrm{~K}\right): \delta 9.48\left(\mathrm{~d},{ }^{1} \mathrm{~J}_{\mathrm{PH}}=507.3 \mathrm{~Hz}, 1 \mathrm{H}, \mathrm{PH}\right), 7.38(\mathrm{~m}$, $2 \mathrm{H}, m-\mathrm{Ph}), 7.31(\mathrm{~m}, 1 \mathrm{H}, p-\mathrm{Ph}), 7.24(\mathrm{~m}, 2 \mathrm{H}, o-\mathrm{Ph}), 7.22\left(\mathrm{~d},{ }^{4} \mathrm{~J}_{\mathrm{PH}}=5.5 \mathrm{~Hz}, 1 \mathrm{H}, 3-\mathrm{CH}\right), 7.07$ (d, $\left.{ }^{4} J_{\mathrm{PH}}=4.4 \mathrm{~Hz}, 1 \mathrm{H}, m-\mathrm{Mes}^{\mathrm{a}}\right), 7.00\left(\mathrm{~d},{ }^{4} J_{\mathrm{PH}}=4.6 \mathrm{~Hz}, 1 \mathrm{H}, m-\mathrm{Mes}^{\mathrm{b}}\right), 6.96\left(\mathrm{~d}, 1 \mathrm{H},{ }^{4} J_{\mathrm{PH}}=3.5 \mathrm{~Hz}\right.$, $\left.m^{\prime}-M_{e s}{ }^{a}\right), 6.81\left(\mathrm{~d},{ }^{4} J_{\mathrm{PH}}=4.4 \mathrm{~Hz}, 1 \mathrm{H}, m^{\prime}-\mathrm{Mes}^{\mathrm{b}}\right.$ ), 3.90 (partial relaxed 1:1:1:1 q, ${ }^{1} J_{\mathrm{BH}} \sim 85 \mathrm{~Hz}$, $1 \mathrm{H}, \mathrm{BH}), 2.67 / 2.20$ (each m, each $\left.1 \mathrm{H}, 9-\mathrm{CH}_{2}\right)^{\mathrm{t}}, 2.54\left(\mathrm{~m}, 2 \mathrm{H}, 6-\mathrm{CH}_{2}\right)^{\mathrm{t}}, 2.34\left(\mathrm{~s}, 3 \mathrm{H}, p-\mathrm{CH}_{3}{ }^{\mathrm{Mes}, \mathrm{a}}\right)$, 2.32 (s, 3H, p- $\mathrm{CH}_{3}{ }^{\mathrm{Mes}, \mathrm{b}}$ ), 2.31 (s, 3H, o- $\mathrm{CH}_{3}{ }^{\mathrm{Mes}, \mathrm{a}}$ ), 2.23 (s, 3H, o- $\mathrm{CH}_{3}{ }^{\mathrm{Mes}, \mathrm{b}}$ ), 2.14 (s, 3H, o'$\left.\mathrm{CH}_{3}{ }^{\text {Mes,b }}\right), 1.91\left(\mathrm{~s}, 3 \mathrm{H}, o^{\prime}-\mathrm{CH}_{3}{ }^{\mathrm{Mes}, \mathrm{a}}\right), 1.69(1 \mathrm{H}), 1.50(2 \mathrm{H}), 1.39(1 \mathrm{H})\left(\text { each } \mathrm{m}, 7,8-\mathrm{CH}_{2}\right)^{\mathrm{t}} \cdot{ }^{\mathrm{t}}$ tentatively assigned]

${ }^{13} \mathrm{C}\left\{{ }^{1} \mathrm{H}\right\}$ NMR (151 MHz, dichloromethane- $\left.d_{2}, 299 \mathrm{~K}\right): \delta 146.5\left(\mathrm{~d},{ }^{4}{ }_{\mathrm{PC}}=3.9 \mathrm{~Hz}, 4-\mathrm{C}\right), 145.2(\mathrm{~d}$, $\left.{ }^{4} J_{\mathrm{PC}}=2.8 \mathrm{~Hz}, p-\mathrm{Mes}^{\mathrm{b}}\right), 144.9\left(\mathrm{~d},{ }^{4} J_{\mathrm{PC}}=3.0 \mathrm{~Hz}, p-\mathrm{Mes}^{\mathrm{a}}\right), 144.4\left(\mathrm{~d},{ }^{2} J_{\mathrm{PC}}=10.1 \mathrm{~Hz}, o^{\prime}-\mathrm{Mes}^{\mathrm{b}}\right)$, $143.6\left(\mathrm{~d},{ }^{2} J_{\mathrm{PC}}=11.8 \mathrm{~Hz}, o_{-}-\mathrm{Mes}^{\mathrm{a}}\right), 143.4\left(\mathrm{~d},{ }^{2} J_{\mathrm{PC}}=8.4 \mathrm{~Hz}, \mathrm{o}^{\prime}-\mathrm{Mes}^{\mathrm{a}}\right), 142.7\left(\mathrm{~d},{ }^{2} \mathrm{~J}_{\mathrm{PC}}=9.9 \mathrm{~Hz}, o^{-}\right.$ Mes $^{\mathrm{b}}$ ), $142.0(\mathrm{~d}, J=0.9 \mathrm{~Hz}, i-\mathrm{Ph}), 141.6\left(\mathrm{~d},{ }^{2} \mathrm{JPC}_{\mathrm{PC}}=14.2 \mathrm{~Hz}, 10-\mathrm{C}\right)^{\mathrm{t}}, 137.4\left(\mathrm{br} \mathrm{d},{ }^{3} J_{\mathrm{PC}}=16.8 \mathrm{~Hz}\right.$, 3-CH), $134.6\left(\mathrm{~d},{ }^{3} J_{\mathrm{PC}}=12.4 \mathrm{~Hz}, 5-\mathrm{C}\right)^{\mathrm{t}}, 132.6\left(\mathrm{~d},{ }^{3} J_{\mathrm{PC}}=11.0 \mathrm{~Hz}, m^{\prime}-\mathrm{Mes}^{\mathrm{a}}\right), 132.1\left(\mathrm{~d},{ }^{3} J_{\mathrm{PC}}=\right.$ $\left.11.5 \mathrm{~Hz}, m^{\prime}-\mathrm{Mes}^{\mathrm{b}}\right), 131.6\left(\mathrm{~d},{ }^{3} J_{\mathrm{PC}}=10.6 \mathrm{~Hz}, m-\mathrm{Mes}^{\mathrm{a}}\right), 130.8\left(\mathrm{~d},{ }^{3} \mathrm{JPC}_{\mathrm{PC}}=10.5 \mathrm{~Hz}, m-\mathrm{Mes}^{\mathrm{b}}\right), 129.4$ (o-Ph), $128.4(m-P h), 127.3(p-P h), 120.5\left(\mathrm{~d},{ }^{1} J_{\mathrm{PC}}=83.1 \mathrm{~Hz}, 1-\mathrm{C}\right), 117.1\left(\mathrm{~d},{ }^{1} \mathrm{JPC}_{\mathrm{PC}}=76.3 \mathrm{~Hz}, i-\right.$ Mes $\left.^{\mathrm{a}}\right), 116.2\left(\mathrm{~d},{ }^{1} J_{\mathrm{PC}}=80.9 \mathrm{~Hz}, i-\mathrm{Mes}^{\mathrm{b}}\right), 32.1\left(\mathrm{~d},{ }^{3} J_{\mathrm{PC}}=7.7 \mathrm{~Hz}, 9-\mathrm{CH}_{2}\right)^{\mathrm{t}}, 28.1\left(\mathrm{~d},{ }^{4} J_{\mathrm{PC}}=1.6 \mathrm{~Hz}\right.$, 
6- $\left.\mathrm{CH}_{2}\right)^{\mathrm{t}}, 22.5\left(7,8-\mathrm{CH}_{2}\right)^{\mathrm{t}}, 22.4\left(\mathrm{~d},{ }^{3} \mathrm{PC}_{\mathrm{PC}}=5.2 \mathrm{~Hz}, o^{\prime}-\mathrm{CH}_{3}{ }^{\mathrm{Mes}, \mathrm{a}}\right), 21.4\left(\mathrm{~d}, J=1.4 \mathrm{~Hz}, p-\mathrm{CH}_{3}{ }^{\mathrm{Mes}, \mathrm{a}}\right)$, $21.3\left(\mathrm{~d},{ }^{3} \mathrm{JPC}_{\mathrm{PC}}=11.5 \mathrm{~Hz}, o-\mathrm{CH}_{3}{ }^{\mathrm{Mes}, \mathrm{a}}\right), 21.2\left(p-\mathrm{CH}_{3}{ }^{\mathrm{Mes}, \mathrm{b}}\right), 21.1\left(\mathrm{br} \mathrm{d},{ }^{3} \mathrm{JPC}_{\mathrm{PC}}=6.1 \mathrm{~Hz}, o^{\prime}-\mathrm{CH}_{3}{ }^{\mathrm{Mes}, \mathrm{b}}\right)$, $21.0\left(\mathrm{~d},{ }^{3} \mathrm{JPC}_{\mathrm{PC}}=11.9 \mathrm{~Hz}, o-\mathrm{CH}_{3}{ }^{\mathrm{Mes}, \mathrm{b}}\right)$, n.o. (2-C).[C $\mathrm{C}_{6} \mathrm{~F}_{5}$ not listed; ${ }^{\mathrm{t}}$ tentatively assigned]

${ }^{31} \mathrm{P}$ NMR (243 MHz, dichloromethane- $\left.d_{2}, 299 \mathrm{~K}\right): \delta-28.5\left(\mathrm{br} \mathrm{d},{ }^{1} \mathrm{~J}_{\mathrm{PH}} \sim 508 \mathrm{~Hz}\right)$.

${ }^{31} \mathbf{P}\left\{{ }^{1} \mathrm{H}\right\}$ NMR $\left(243 \mathrm{MHz}\right.$, dichloromethane- $\left.d_{2}, 299 \mathrm{~K}\right): \delta-28.5(\mathrm{~m})$.

${ }^{11}$ B NMR (192 MHz, dichloromethane- $\left.d_{2}, 299 \mathrm{~K}\right): \delta-20.8\left(\mathrm{~d},{ }^{1} J_{\mathrm{BH}} \sim 83 \mathrm{~Hz}\right)$

${ }^{11} \mathbf{B}\left\{{ }^{1} \mathrm{H}\right\}$ NMR $\left(192 \mathrm{MHz}\right.$, dichloromethane- $\left.d_{2}, 299 \mathrm{~K}\right): \delta-20.8\left(v_{1 / 2} \sim 55 \mathrm{~Hz}\right)$

${ }^{19} \mathrm{~F}$ NMR $\left(564 \mathrm{MHz}\right.$, dichloromethane- $\left.d_{2}, 299 \mathrm{~K}\right): \delta-130.9(\mathrm{~m}, 2 \mathrm{~F}, 0),-163.2\left(\mathrm{t},{ }^{3} J_{\mathrm{FF}}=20.1 \mathrm{~Hz}\right.$, $1 \mathrm{~F}, p),-166.9(\mathrm{~m}, 2 \mathrm{~F}, m)\left(\mathrm{C}_{6} \mathrm{~F}_{5}\right)\left[\Delta \delta^{19} \mathrm{~F}_{\mathrm{mp}}=3.7\right],-133.0(\mathrm{~m}, 2 \mathrm{~F}, o),-162.7\left(\mathrm{t},{ }^{3} J_{\mathrm{FF}}=20.1 \mathrm{~Hz}, 1 \mathrm{~F}\right.$, p), $-166.3(\mathrm{~m}, 2 \mathrm{~F}, m)\left(\mathrm{C}_{6} \mathrm{~F}_{5}\right)\left[\Delta \delta^{19} \mathrm{~F}_{\mathrm{mp}}=3.6\right]$.
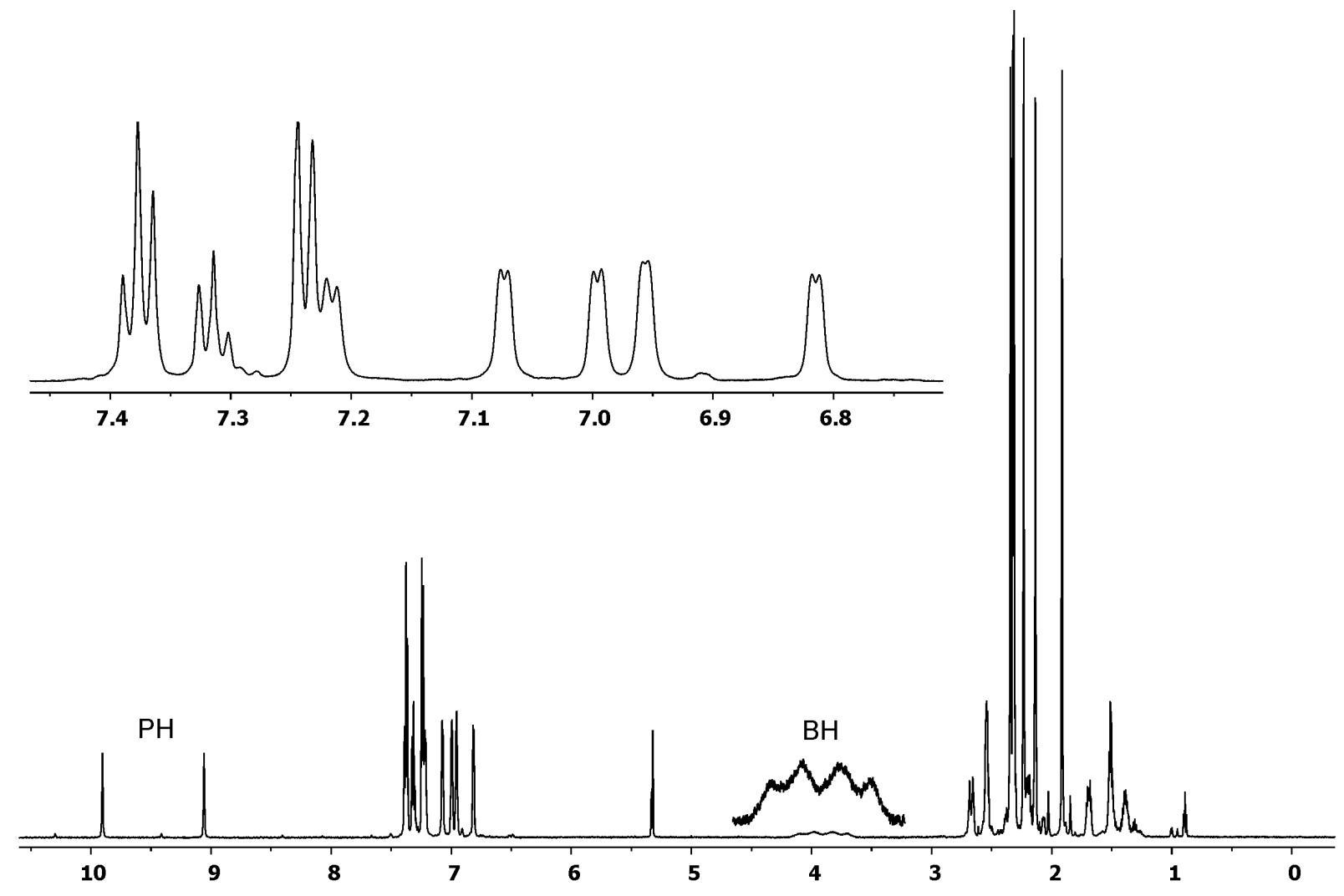

Figure S41: ${ }^{1}$ H NMR (600 MHz, dichloromethane- $\left.d_{2}, 299 \mathrm{~K}\right)$ spectrum of compound 14 

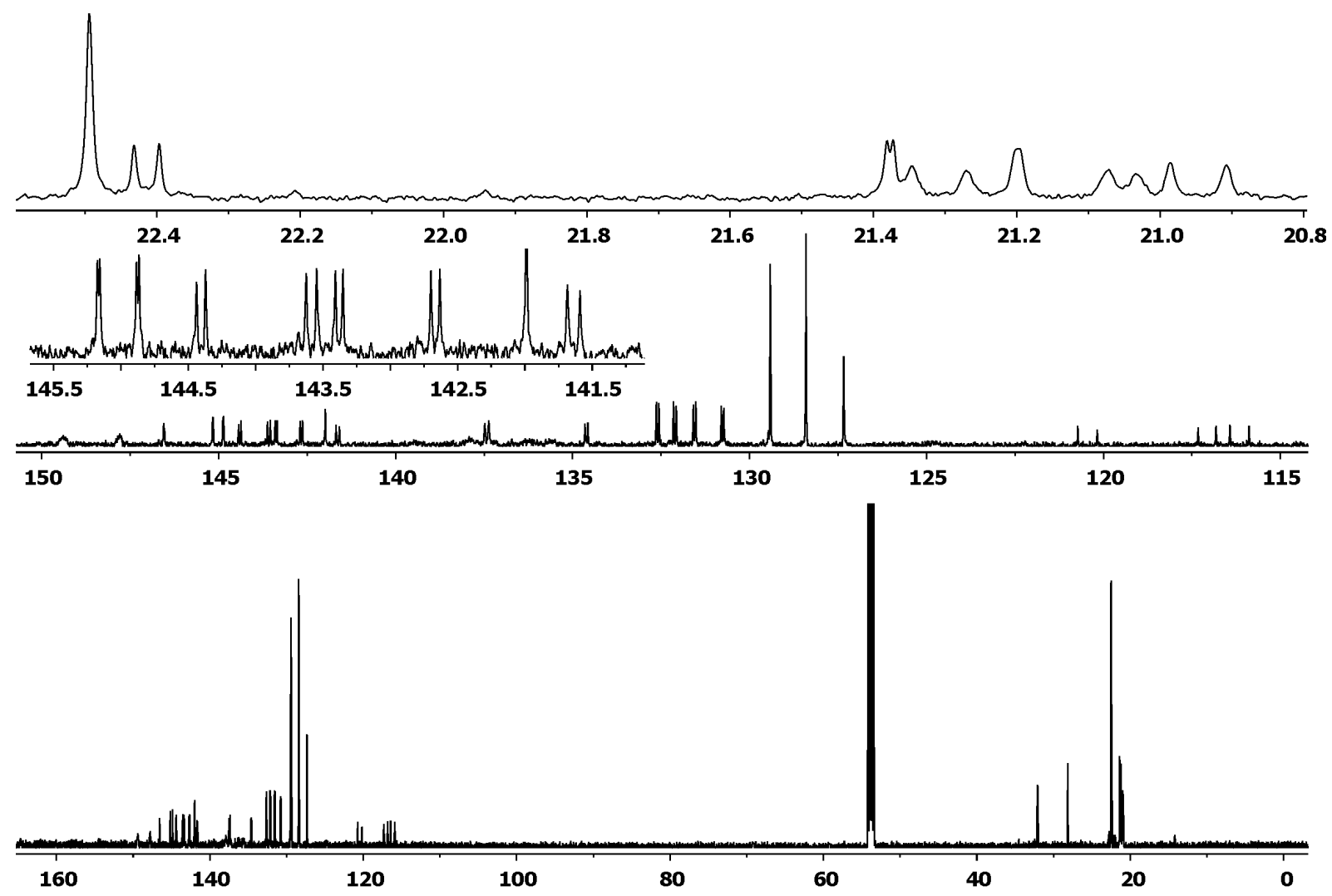

Figure S42: ${ }^{13} \mathbf{C}\left\{{ }^{1} \mathbf{H}\right\}$ NMR (151 MHz, dichloromethane- $\left.d_{2}, 299 \mathrm{~K}\right)$ spectrum of compound $\mathbf{1 4}$

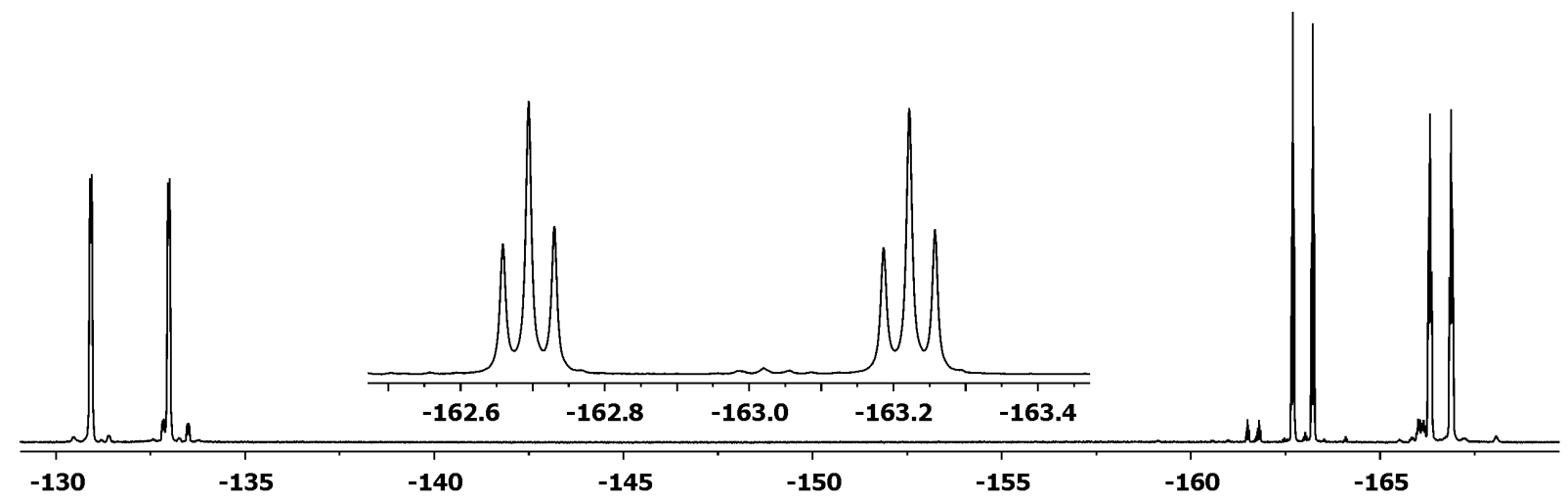

Figure S43: ${ }^{19} \mathrm{~F}$ NMR $\left(564 \mathrm{MHz}\right.$, dichloromethane- $\left.d_{2}, 299 \mathrm{~K}\right)$ spectrum of compound 14

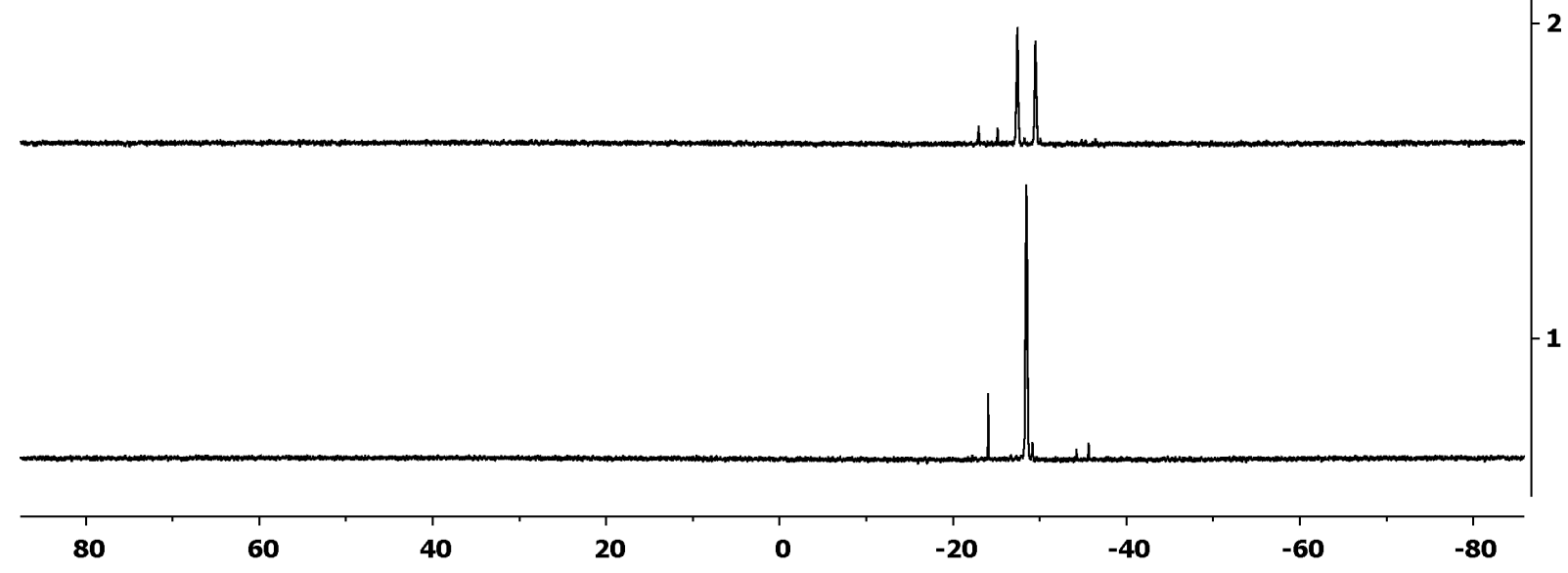

Figure S44: ${ }^{31} \mathrm{P}\left\{{ }^{1} \mathrm{H}\right\}$ NMR (1) and ${ }^{31} \mathrm{P}$ NMR (2) (243 MHz, dichloromethane- $\left.d_{2}, 299 \mathrm{~K}\right)$ spectra of compound 14 


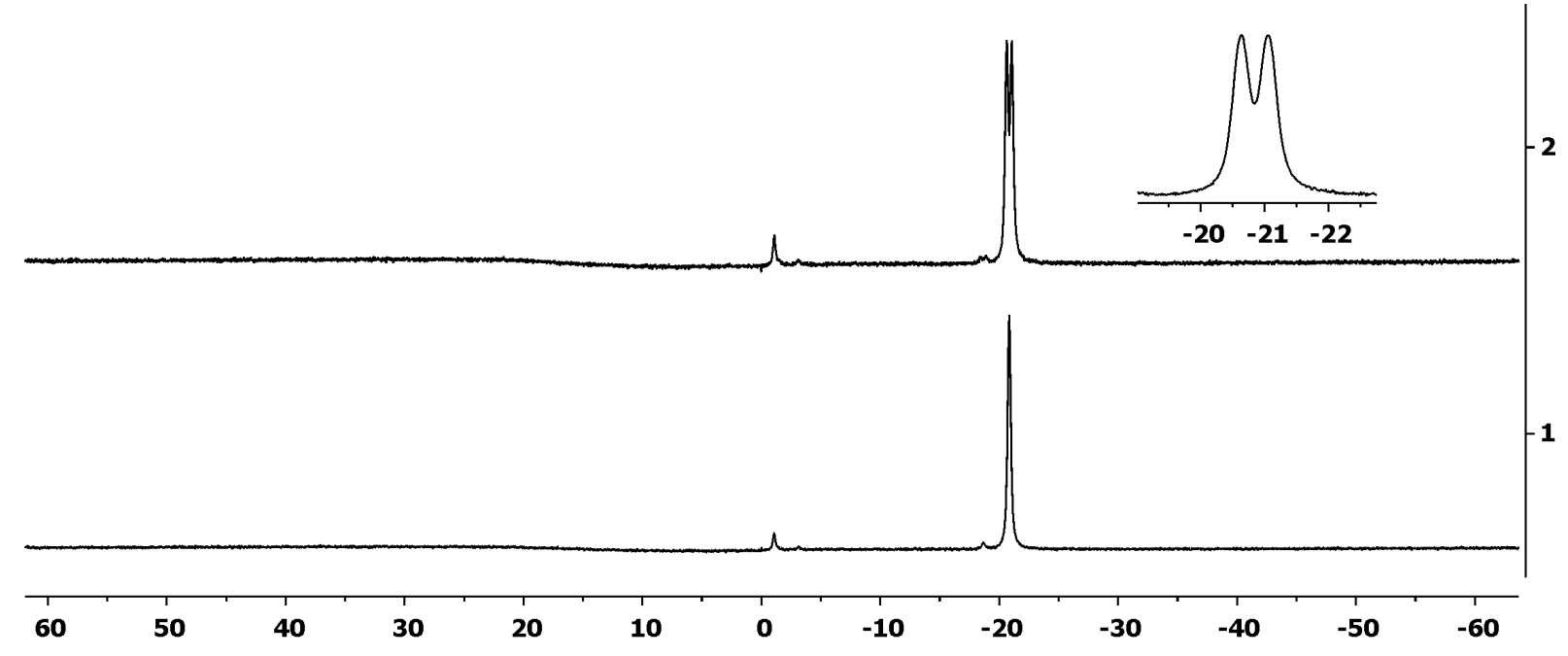

Figure S45: ${ }^{11} \mathrm{~B}\left\{{ }^{1} \mathrm{H}\right\}$ NMR (1) and ${ }^{11} \mathrm{~B}$ NMR (2) (192 MHz, dichloromethane- $\left.d_{2}, 299 \mathrm{~K}\right)$ spectra of compound 14

Crystals suitable for the X-ray crystal structure analysis were obtained from a solution of compound 14 in dichloromethane at $-35^{\circ} \mathrm{C}$ :

X-ray crystal structure analysis of compound 14: formula $\mathrm{C}_{46} \mathrm{H}_{38} \mathrm{BF}_{10} \mathrm{P} \cdot \mathrm{CH}_{2} \mathrm{Cl}_{2}, M=$ 907.47, colourless crystal, $0.07 \times 0.06 \times 0.04 \mathrm{~mm}, a=11.7476(3), b=13.4169(3), c=$ 15.7177(5) $\AA, \alpha=106.742(1), \beta=103.703(1), y=107.436(2)^{\circ}, V=2117.0(1) \AA^{3}, \rho_{\text {calc }}=1.424$ $\mathrm{gcm}^{-3}, \mu=0.270 \mathrm{~mm}^{-1}$, empirical absorption correction (0.981 $\left.\leq \mathrm{T} \leq 0.989\right), Z=2$, triclinic, space group $P^{\overline{1}}$ (No. 2), $\lambda=0.71073 \AA, T=223(2) \mathrm{K}, \omega$ and $\varphi$ scans, 10482 reflections collected $( \pm h, \pm k, \pm), 7274$ independent $\left(R_{\text {int }}=0.038\right)$ and 4952 observed reflections $[>2 \sigma(I)]$, 592 refined parameters, $R=0.085, w R^{2}=0.177$, max. (min.) residual electron density 0.40 ($0.29) e . \AA^{-3}$. The hydrogen atoms at $\mathrm{P} 1$ and $\mathrm{B} 1$ were refined freely; others were calculated and refined as riding atoms. 


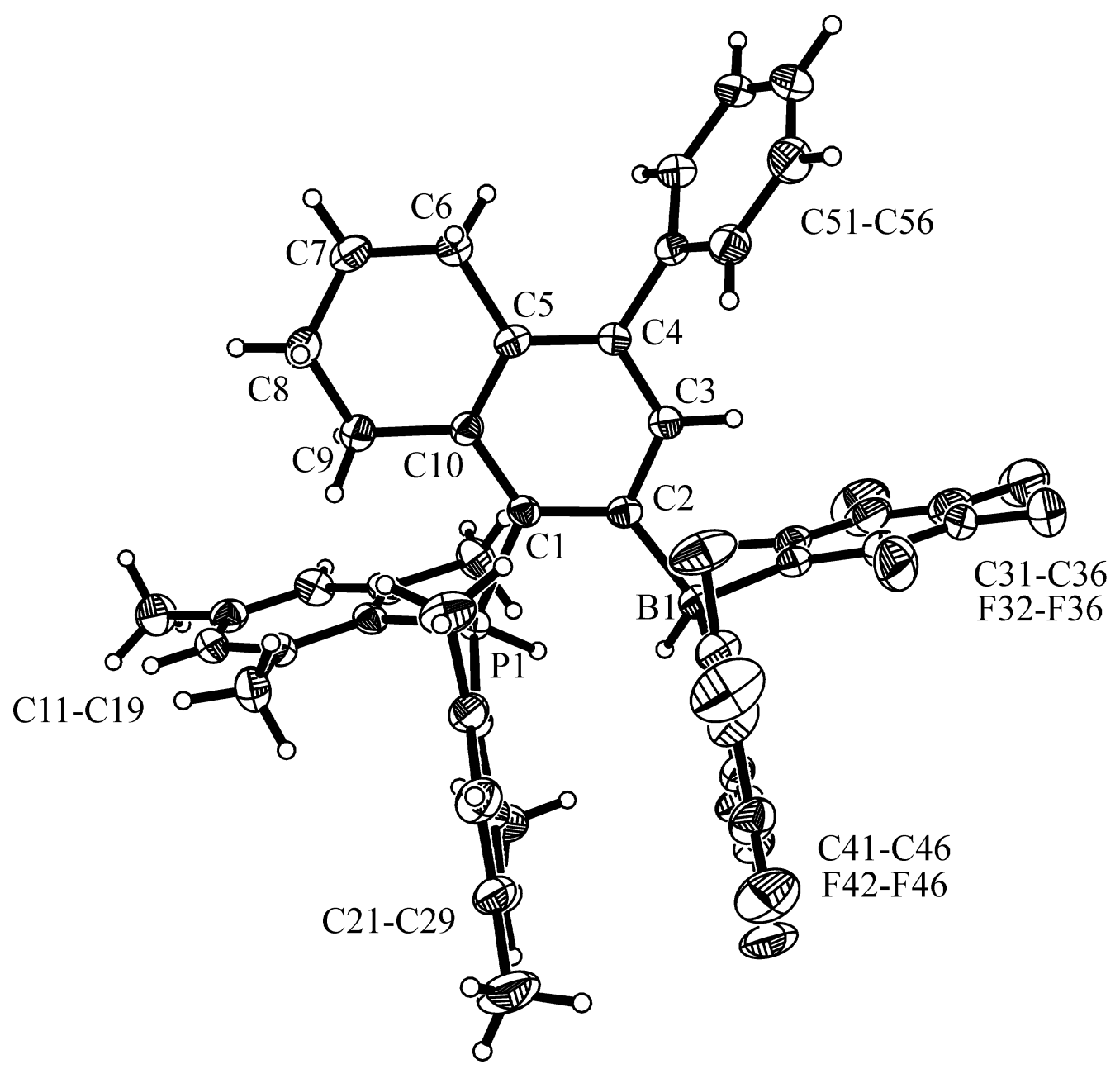

Figure S46: X-ray crystal structure of compound 14 (thermal ellipsoids are shown at the $30 \%$ probability level)

\section{Generation of compound 14-D2}
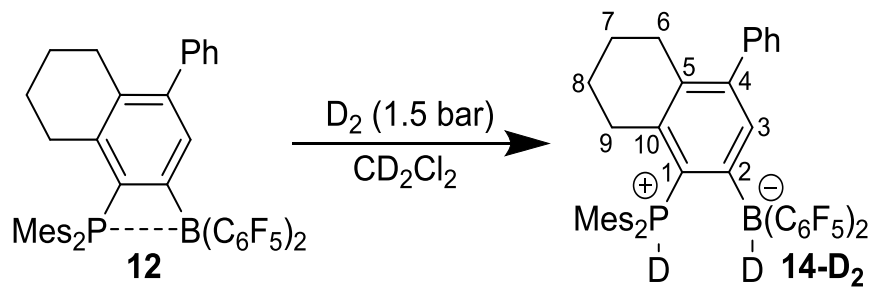

\section{Scheme S8}

A yellow solution of compound $12(20.0 \mathrm{mg}, 24.4 \mu \mathrm{mol}, 1.0 \mathrm{eq})$ in $\mathrm{CD}_{2} \mathrm{Cl}_{2}(0.5 \mathrm{~mL})$ was degassed at $-78^{\circ} \mathrm{C}$ using a J-Young NMR tube. Then the solution was exposed to a deuterium gas atmosphere (1.5 bar). After shaking the tube for $16 \mathrm{~h}$ at room temperature, the reaction mixture was characterized by NMR experiments.

A mixture of compounds 12 and 14- $\mathbf{D}_{2}\left(\mathbf{1 2}: \mathbf{1 4}-\mathrm{D}_{\mathbf{2}} \sim 54: 46\left({ }^{31} \mathrm{P}\left\{{ }^{1} \mathrm{H}\right\}\right)\right.$ was observed. 
The NMR data of compounds $14-D_{2}$ and 12 are consistent with those listed for isolated compounds 14 and 12 (see above).

\section{Compound 14- $\mathrm{D}_{2}$}

${ }^{2}$ H NMR (92 MHz, dichloromethane, $\left.299 \mathrm{~K}\right): \delta 9.46\left(\mathrm{~d},{ }^{1} \mathrm{JPD}_{\mathrm{PD}}=77.1 \mathrm{~Hz}, 1 \mathrm{D}, \mathrm{PD}\right), 3.88$ (br, 1D, BD).

${ }^{31} \mathrm{P}$ NMR $\left(243 \mathrm{MHz}\right.$, dichloromethane- $\left.d_{2}, 299 \mathrm{~K}\right): \delta-28.6$ (br 1:1:1 t, $\left.{ }^{1} \mathrm{JPD}_{\mathrm{PD}} \sim 79 \mathrm{~Hz}\right)$.

${ }^{31} \mathrm{P}\left\{{ }^{1} \mathrm{H}\right\}$ NMR $\left(243 \mathrm{MHz}\right.$, dichloromethane- $\left.d_{2}, 299 \mathrm{~K}\right): \delta-28.6\left(\right.$ br 1:1:1 t, $\left.{ }^{1} \mathrm{JPD}_{\mathrm{PD}} \sim 79 \mathrm{~Hz}\right)$.

${ }^{11}$ B NMR (192 MHz, dichloromethane- $\left.d_{2}, 299 \mathrm{~K}\right): \delta-21.0\left(v_{1 / 2} \sim 70 \mathrm{~Hz}\right)$

${ }^{11} \mathbf{B}\left\{{ }^{1} \mathrm{H}\right\}$ NMR $\left(192 \mathrm{MHz}\right.$, dichloromethane- $\left.d_{2}, 299 \mathrm{~K}\right): \delta-21.0\left(v_{1 / 2} \sim 70 \mathrm{~Hz}\right)$

${ }^{19} \mathrm{~F}$ NMR $\left(564 \mathrm{MHz}\right.$, dichloromethane- $\left.d_{2}, 299 \mathrm{~K}\right): \delta-131.0,-133.0$ (each $\mathrm{m}$, each $2 \mathrm{~F}, 0$ ), - 162.7, $-163.3\left(\right.$ each $t,{ }^{3} J_{F F}=20.1 \mathrm{~Hz}$, each $\left.1 \mathrm{~F}, p\right),-166.3,-166.9($ each $\mathrm{m}$, each $2 \mathrm{~F}, m)\left(\mathrm{C}_{6} \mathrm{~F}_{5}\right)$.

isolated compound 12

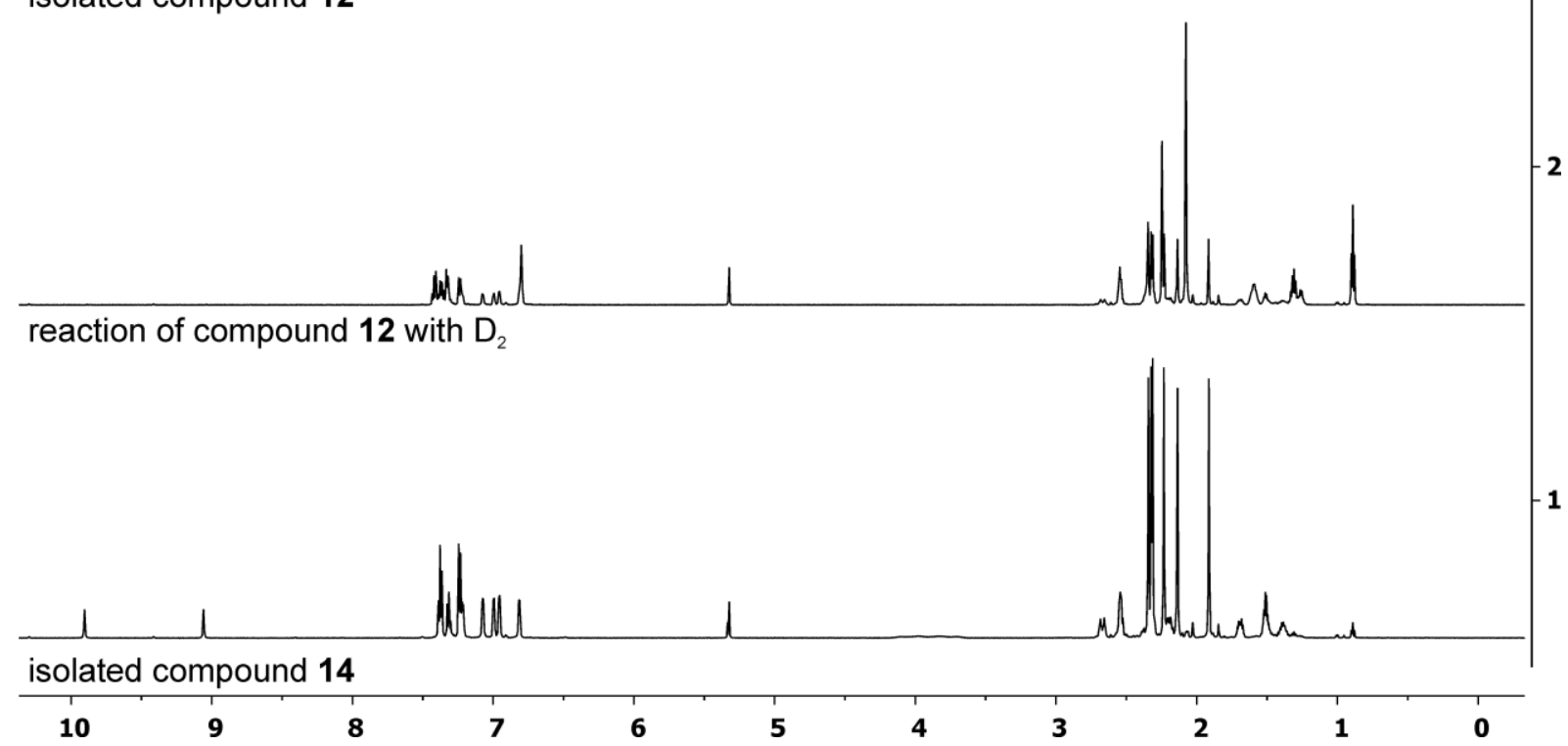

Figure S47: ${ }^{1} \mathbf{H}$ NMR $\left(600 \mathrm{MHz}\right.$, dichloromethane- $\left.d_{2}, 299 \mathrm{~K}\right)$ spectra of (1) the isolated compound 14 and (2) the reaction of compound 12 with $\mathrm{D}_{2}$; (3) ${ }^{1} \mathrm{H}$ NMR $\left(500 \mathrm{MHz}\right.$, dichloromethane- $\left.d_{2}, 299 \mathrm{~K}\right)$ spectrum of the isolated compound 12. 


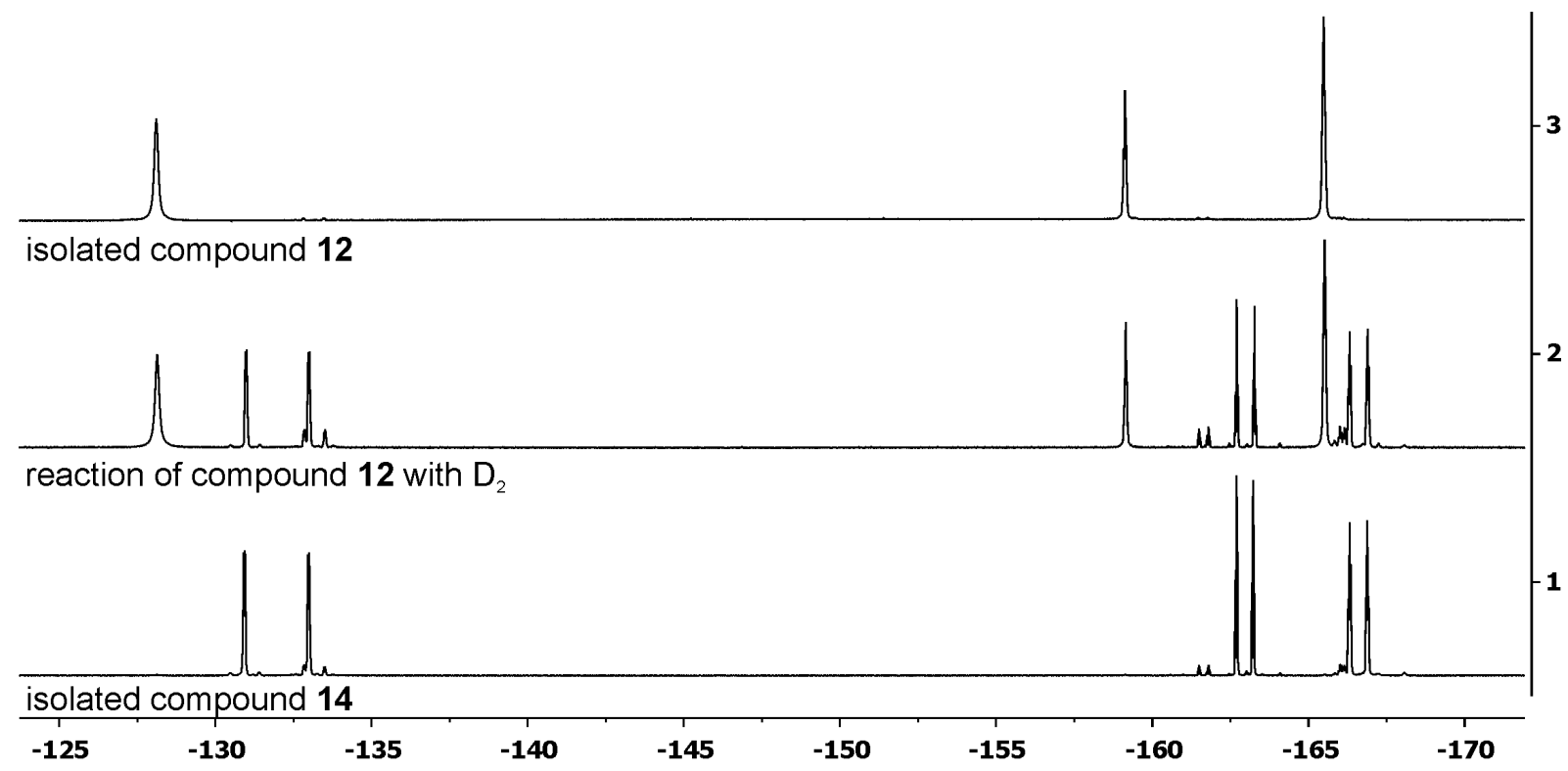

Figure S48: ${ }^{19} \mathrm{~F}$ NMR $\left(564 \mathrm{MHz}\right.$, dichloromethane- $\left.d_{2}, 299 \mathrm{~K}\right)$ spectra of (1) the isolated compound 14 and (2) the reaction of compound 12 with $\mathrm{D}_{2} ;(3){ }^{19} \mathrm{~F}$ NMR $(470 \mathrm{MHz}$, dichloromethane-d2, $299 \mathrm{~K})$ spectrum the isolated compound 12.

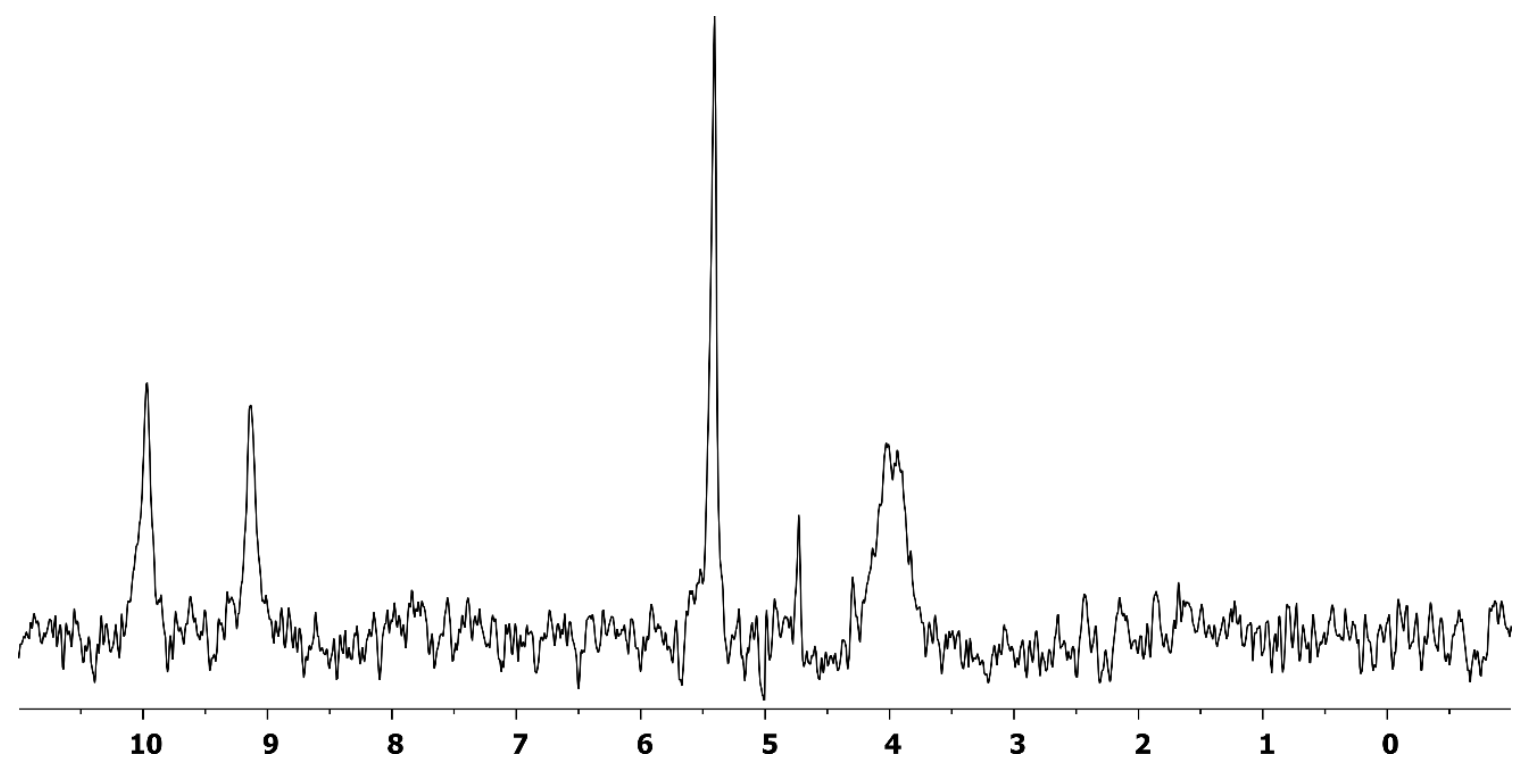

Figure S49: ${ }^{2} \mathrm{H}$ NMR (92 MHz, dichloromethane, $299 \mathrm{~K}$ ) spectrum of the reaction compound 12 with $\mathrm{D}_{2}$ 


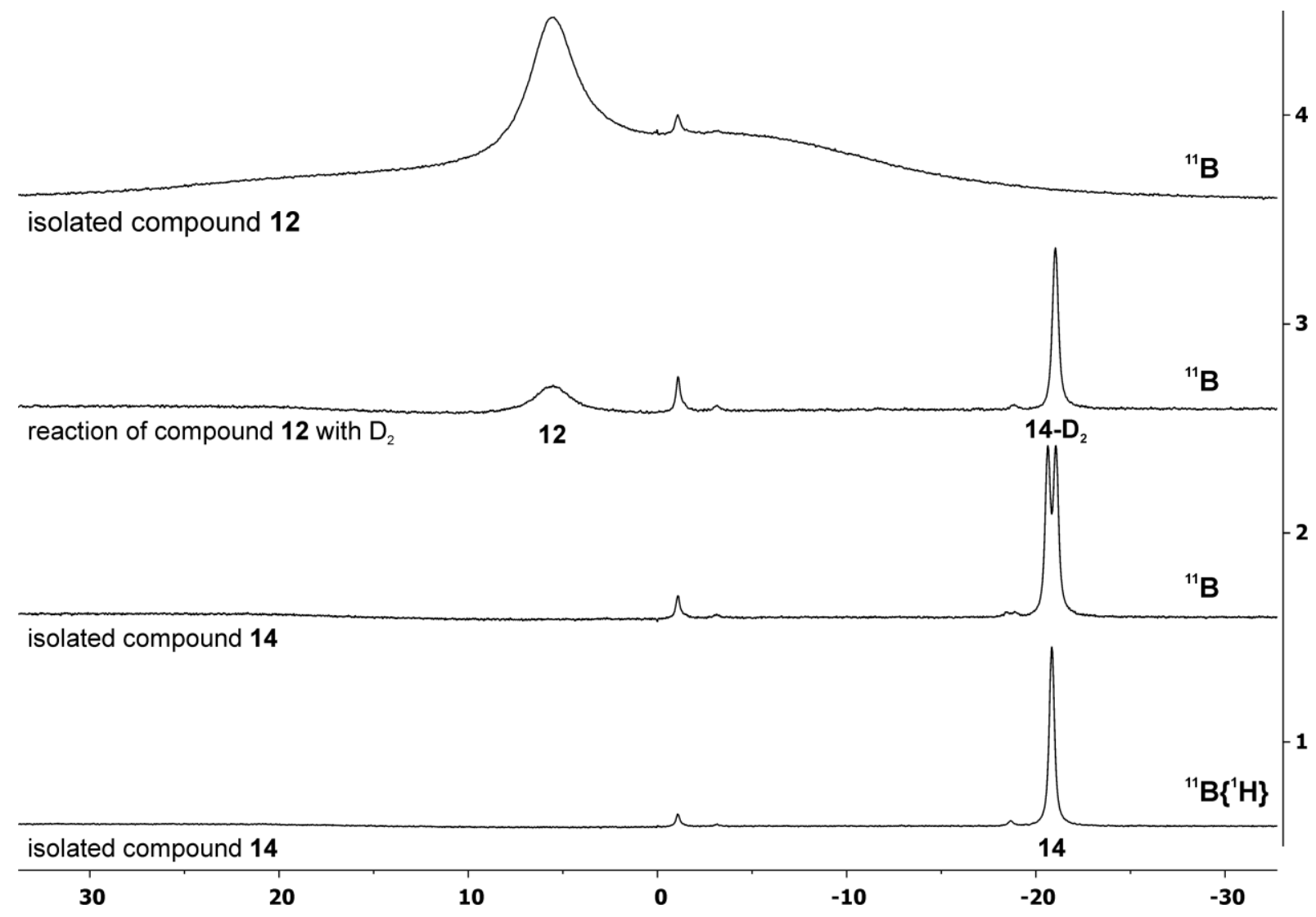

Figure S50: (1) ${ }^{11} \mathbf{B}\left\{{ }^{1} \mathrm{H}\right\}$ and (2) ${ }^{11} \mathbf{B}$ NMR (192 MHz, dichloromethane- $d_{2}, 299 \mathrm{~K}$ ) spectra of the isolated compound 14; (3) ${ }^{11} \mathrm{~B}$ NMR (192 MHz, dichloromethane- $d_{2}, 299 \mathrm{~K}$ ) spectrum of the reaction of compound 12 with $\mathrm{D}_{2}$; (4) ${ }^{11} \mathrm{~B}$ NMR (160 MHz, dichloromethane- $d_{2}, 299 \mathrm{~K}$ ) spectrum of the isolated compound 12

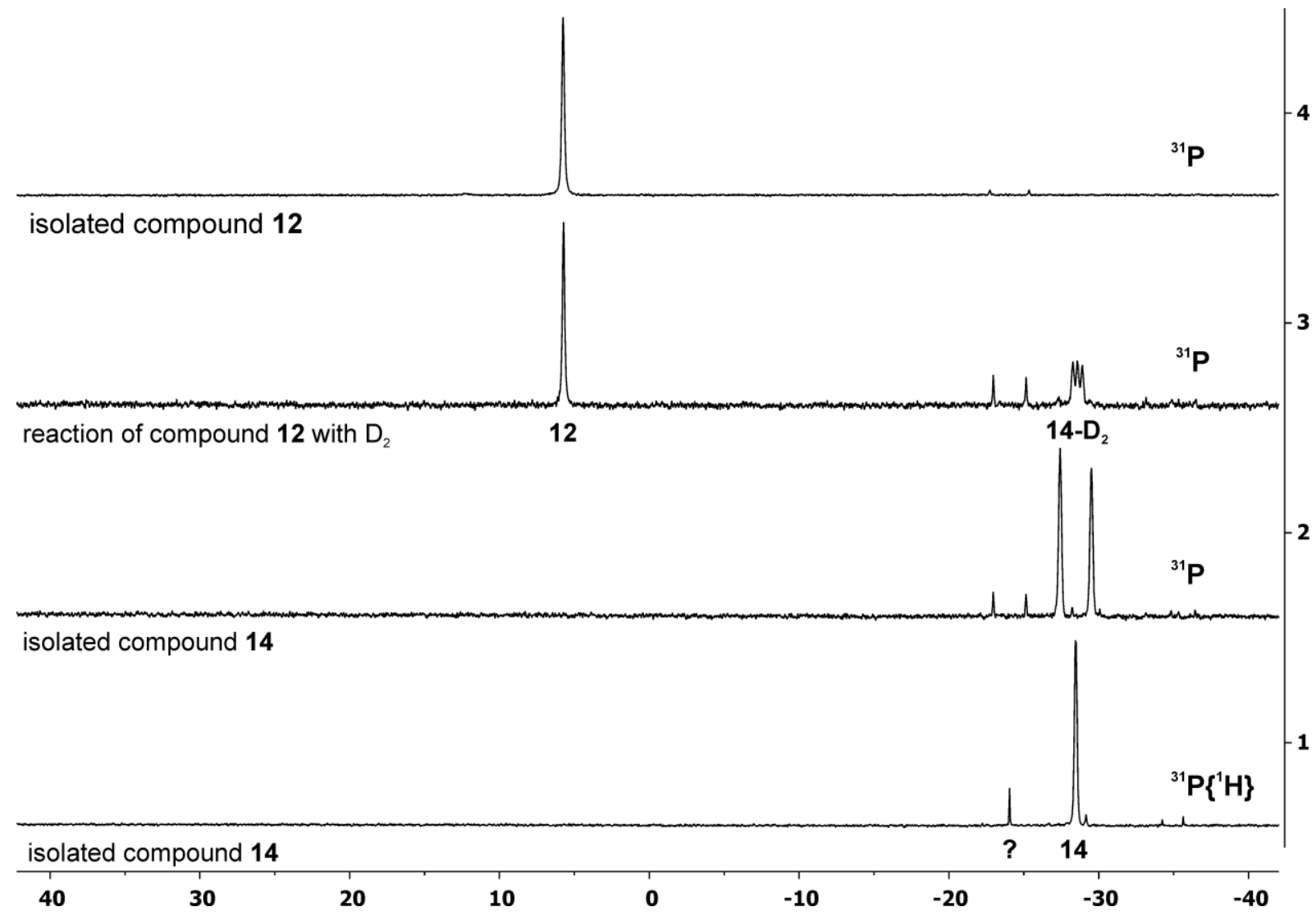

Figure S51: (1) ${ }^{31} \mathbf{P}\left\{{ }^{1} \mathrm{H}\right\}$ and (2) ${ }^{31} \mathbf{P}$ NMR $\left(243 \mathrm{MHz}\right.$, dichloromethane- $\left.d_{2}, 299 \mathrm{~K}\right)$ spectra of the isolated compound 14; (3) ${ }^{31} \mathbf{P}$ NMR $\left(243 \mathrm{MHz}\right.$, dichloromethane- $\left.d_{2}, 299 \mathrm{~K}\right)$ spectrum of the reaction of compound 12 with $\mathrm{D}_{2}$; (5) ${ }^{31} \mathrm{P}$ NMR (202 MHz, dichloromethane- $d_{2}, 299 \mathrm{~K}$ ) spectrum of the isolated compound 12 


\section{Synthesis of compound 17}

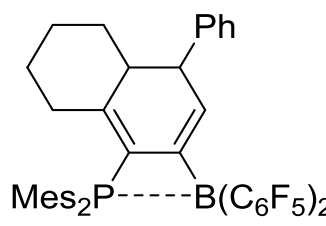

11

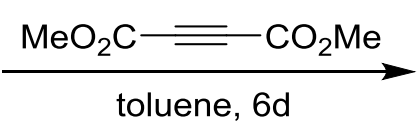

toluene, 6d

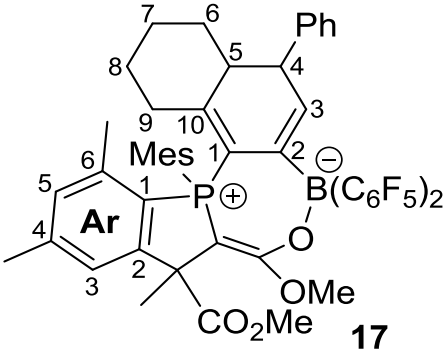

Scheme S9

A solution of dimethylacetylenedicarboxylate $(17.9 \mu \mathrm{L}, 20.7 \mathrm{mg}, 145.9 \mu \mathrm{mol}, 1.0 \mathrm{eq})$ in toluene $(1 \mathrm{~mL})$ was added to a solution of compound 11 (120.0 mg, $145.9 \mu \mathrm{mol}, 1.0 \mathrm{eq})$ in toluene $(2 \mathrm{~mL})$. The orange reaction mixture was stirred at $75^{\circ} \mathrm{C}$ for 6 days. Then all volatiles were removed in vacuo and the resulting sticky residue was dissolved in pentane $(5 \mathrm{~mL})$, which was subsequently removed in vacuo. After adding pentane $(3 \mathrm{~mL})$ to the obtained sticky solid the resulting suspension was filtered via cannula (Whatman glass fiber filter). The remaining solid was washed with pentane $(5 \times 3 \mathrm{~mL})$ and dried in vacuo to give compound 17 as a pale yellow solid (70.8 mg, $73.4 \mu \mathrm{mol}, 50 \%)$.

IR $(\mathrm{KBr}): \tilde{v}\left[\mathrm{~cm}^{-1}\right]=3448(\mathrm{w}), 3193(\mathrm{w}), 3121(\mathrm{w}), 3604(\mathrm{w}), 3029(\mathrm{~m}), 2991(\mathrm{~m}), 2942(\mathrm{~m})$, $2862(\mathrm{~m}), 2788(w), 2735(w), 2688(w), 2390(w), 2087(w), 1946(w), 1872(w), 1792(w)$, $1736(\mathrm{~s}), 1683(\mathrm{w}), 1643(\mathrm{~m}), 1605(\mathrm{~m}), 1558(\mathrm{w}), 1515(\mathrm{~m}), 1451(\mathrm{~s}), 1041(\mathrm{w}), 1377(\mathrm{~m}), 1341$ (m), $1276(\mathrm{~m}), 1248(\mathrm{~m}), 1206(\mathrm{~m}), 1180(\mathrm{w}), 1148(\mathrm{w}), 1088(\mathrm{~s}), 1030(\mathrm{~m}), 972(\mathrm{~s}), 926(\mathrm{w})$, $859(\mathrm{~m}), 801(\mathrm{w}), 783(\mathrm{~m}), 741(\mathrm{~m}), 691(\mathrm{~s}), 658(\mathrm{~m}), 642(\mathrm{~s}), 616(\mathrm{w}), 654(\mathrm{~m}), 525(\mathrm{w}), 460$ (w), $435(w)$.

M.p. $259^{\circ} \mathrm{C}$.

Anal. Calc. for $\mathrm{C}_{52} \mathrm{H}_{44} \mathrm{BF}_{10} \mathrm{O}_{4} \mathrm{P}: \mathrm{C}: 64.74 ; \mathrm{H}: 4.60$. Found: $\mathrm{C}: 64.99 ; \mathrm{H}: 4.73$.

A solution of the yellow solid in dichloromethane- $d_{2}$ showed at $299 \mathrm{~K}$ a mixture of two compounds, the major component was assigned as compound $\mathbf{1 7}$, the minor one was not identified yet [ratio ca. $\left.64: 36\left({ }^{31} \mathrm{P}\right)\right]$.

$[\mathrm{Ar}=$ 4,6-dimethylphenylene $]$

Major component 17:

${ }^{1} \mathbf{H}$ NMR $\left(600 \mathrm{MHz}\right.$, dichloromethane- $\left.d_{2}, 299 \mathrm{~K}\right): \delta 7.23(\mathrm{~m}, 2 \mathrm{H}, m-\mathrm{Ph}), 7.17(\mathrm{~m}, 1 \mathrm{H}, p-\mathrm{Ph})$, $7.11\left(\mathrm{~m}, 1 \mathrm{H}, 3-\mathrm{CH}^{\mathrm{Ar}}\right), 7.00(\mathrm{~m}, 2 \mathrm{H}, \mathrm{o}-\mathrm{Ph}), 6.97\left(\mathrm{br} \mathrm{d},{ }^{4} \mathrm{~J}_{\mathrm{PH}}=5.5 \mathrm{~Hz}, 1 \mathrm{H}, 5-\mathrm{CH}^{\mathrm{Ar}}\right), 6.75(\mathrm{br} \mathrm{d}$, $\left.{ }^{4} J_{\mathrm{PH}}=2.7 \mathrm{~Hz}, 1 \mathrm{H}, m-\mathrm{Mes}\right), 6.57\left(\mathrm{br} \mathrm{d},{ }^{4} J_{\mathrm{PH}}=3.9 \mathrm{~Hz}, 1 \mathrm{H}, m^{\prime}-\mathrm{Mes}\right), 5.86(\mathrm{~m}, 1 \mathrm{H}, 3-\mathrm{CH}), 3.95$ $\left(\mathrm{dm},{ }^{3} \mathrm{~J}_{\mathrm{HH}}=10.8 \mathrm{~Hz}, 1 \mathrm{H}, 4-\mathrm{CH}\right), 3.68\left(\mathrm{~s}, 3 \mathrm{H}, \mathrm{OMe}^{\mathrm{C}=\mathrm{O}}\right), 3.49$ (s, 3H, OMe), $2.72\left(\mathrm{~s}, 3 \mathrm{H}, o^{\prime}-\right.$ $\mathrm{CH}_{3}{ }^{\mathrm{Mes}}$ ), 2.55/2.06 (each m, each 1H, 9- $\mathrm{CH}_{2}$ ), 2.35 (s, 3H, 4- $\mathrm{CH}_{3}{ }^{\mathrm{Ar}}$ ), $2.35(\mathrm{~m}, 1 \mathrm{H}, 5-\mathrm{CH}), 2.25$ 
(s, 3H, o- $\mathrm{CH}_{3}{ }^{\mathrm{Mes}}$ ), 2.20 (s, 3H, p- $\mathrm{CH}_{3}{ }^{\mathrm{Mes}}$ ), 2.07 (s, 3H, 6- $\mathrm{CH}_{3}{ }^{\mathrm{Ar}}$ ), 1.91 (s, 3H, $\left.\mathrm{CH}_{3}\right), 1.53,1.16$ (each m, each $1 \mathrm{H}, 7-\mathrm{CH}_{2}$ ), 1.33/1.02 (each m, each $1 \mathrm{H}, 6-\mathrm{CH}_{2}$ ), 1.30/0.94 (each m, each $1 \mathrm{H}$, 8- $\mathrm{CH}_{2}$ ).

${ }^{13} \mathrm{C}\left\{{ }^{1} \mathrm{H}\right\}$ NMR $\left(151 \mathrm{MHz}\right.$, dichloromethane- $\left.d_{2}, 299 \mathrm{~K}\right): \delta 174.7\left(\mathrm{~d},{ }^{3} \mathrm{JPC}_{\mathrm{PC}}=6.9 \mathrm{~Hz}, \mathrm{O}=\mathrm{C}\right), 165.8$ $\left(\mathrm{d},{ }^{2} \mathrm{JPC}_{\mathrm{PC}}=18.3 \mathrm{~Hz}, \mathrm{O}_{2} \mathrm{C}=\right), 154.6\left(\mathrm{~d},{ }^{2} \mathrm{~J}_{\mathrm{PC}}=21.8 \mathrm{~Hz}, 2-\mathrm{C}^{\mathrm{Ar}}\right), 148.4\left(\mathrm{~d},{ }^{2} \mathrm{~J}_{\mathrm{PC}}=6.7 \mathrm{~Hz}, 10-\mathrm{C}\right), 145.9$ (d, $\left.{ }^{2} J_{\mathrm{PC}}=10.0 \mathrm{~Hz}, o-M e s\right), 145.7\left(\mathrm{~d},{ }^{4} J_{\mathrm{PC}}=2.7 \mathrm{~Hz}, 4-\mathrm{C}^{\mathrm{Ar}}\right.$ ), $145.2\left(\mathrm{~d},{ }^{2} \mathrm{~J}_{\mathrm{PC}}=12.7 \mathrm{~Hz}, o^{\prime}-\mathrm{Mes}\right)$, $144.1\left(\mathrm{~d},{ }^{4} J_{\mathrm{PC}}=2.9 \mathrm{~Hz}, p-\mathrm{Mes}\right), 142.8(i-\mathrm{Ph}), 141.8\left(\mathrm{~d},{ }^{2} J_{\mathrm{PC}}=10.3 \mathrm{~Hz}, 6-\mathrm{C}^{\mathrm{Ar}}\right), 140.3$ (br, 2-C), $138.6(\mathrm{br}, 3-\mathrm{CH}), 133.0\left(\mathrm{~d},{ }^{3} J_{\mathrm{PC}}=10.3 \mathrm{~Hz}, 5-\mathrm{CH}^{\mathrm{Ar}}\right), 132.1$ (d, $\left.{ }^{3} J_{\mathrm{PC}}=12.3 \mathrm{~Hz}, m^{\prime}-\mathrm{Mes}\right), 131.9$ $\left(\mathrm{d},{ }^{3} \mathrm{JPC}=11.0 \mathrm{~Hz}, m\right.$-Mes), $129.1(o-\mathrm{Ph}), 128.5(m-\mathrm{Ph}), 126.5(p-\mathrm{Ph}), 124.6\left(\mathrm{~d},{ }^{1} J_{\mathrm{PC}}=81.6 \mathrm{~Hz}\right.$, $1-\mathrm{C}), 123.9\left(\mathrm{~d},{ }^{1} J_{\mathrm{PC}}=86.5 \mathrm{~Hz}, 1-\mathrm{C}^{\mathrm{Ar}}\right), 123.6\left(\mathrm{~d},{ }^{3} \mathrm{JPC}_{\mathrm{PC}}=10.2 \mathrm{~Hz}, 3-\mathrm{CH}^{\mathrm{Ar}}\right), 117.0\left(\mathrm{~d},{ }^{1} \mathrm{~J}_{\mathrm{PC}}=\right.$ $82.0 \mathrm{~Hz}, i-M e s), 71.8$ (d, $\left.{ }^{1} J_{P C}=116.9 \mathrm{~Hz}, \mathrm{PC}=\right), 56.7$ (d, $\left.{ }^{2} J_{\mathrm{PC}}=15.3 \mathrm{~Hz}, \mathrm{CMe}\right), 53.7(\mathrm{OMe})$, $52.4\left(\mathrm{OMe}^{\mathrm{C}=\mathrm{O}}\right), 48.4\left(\mathrm{~d},{ }^{3} \mathrm{~J}_{\mathrm{PC}}=13.0 \mathrm{~Hz}, 5-\mathrm{CH}\right), 43.8\left(\mathrm{~d},{ }^{4} \mathrm{~J}_{\mathrm{PC}}=2.1 \mathrm{~Hz}, 4-\mathrm{CH}\right), 33.5\left(\mathrm{~d},{ }^{3} \mathrm{~J}_{\mathrm{PC}}=\right.$ $\left.9.4 \mathrm{~Hz}, 9-\mathrm{CH}_{2}\right), 29.7\left(\mathrm{CH}_{3}\right), 29.5\left(\mathrm{~m}, 6-\mathrm{CH}_{2}\right), 28.6\left(\mathrm{~d}, J=1.5 \mathrm{~Hz}, 8-\mathrm{CH}_{2}\right), 26.6\left(7-\mathrm{CH}_{2}\right), 24.9$ $\left(\mathrm{m}, \mathrm{o}^{\prime}-\mathrm{CH}_{3}{ }^{\mathrm{Mes}}\right), 23.8\left(\mathrm{~d},{ }^{3} \mathrm{JPC}=6.8 \mathrm{~Hz}, \mathrm{o}^{-} \mathrm{CH}_{3}{ }^{\mathrm{Mes}}\right), 21.7\left(\mathrm{~d}, J=1.3 \mathrm{~Hz}, 4-\mathrm{CH}_{3}{ }^{\mathrm{Ar}}\right), 20.9\left(\mathrm{~d},{ }^{3} J_{\mathrm{PC}}=\right.$ $\left.3.7 \mathrm{~Hz}, 6-\mathrm{CH}_{3}{ }^{\mathrm{Ar}}\right), 20.7$ (p- $\left.\mathrm{CH}_{3}{ }^{\mathrm{Mes}}\right)$. [ $\mathrm{C}_{6} \mathrm{~F}_{5}$ not listed]

${ }^{31} \mathrm{P}\left\{{ }^{1} \mathrm{H}\right\}$ NMR $\left(243 \mathrm{MHz}\right.$, dichloromethane- $\left.d_{2}, 299 \mathrm{~K}\right): \delta 15.9\left(v_{1 / 2} \sim 4 \mathrm{~Hz}\right)$.

${ }^{11} \mathbf{B}\left\{{ }^{1} \mathrm{H}\right\}$ NMR $\left(192 \mathrm{MHz}\right.$, dichloromethane- $\left.d_{2}, 299 \mathrm{~K}\right): \delta 0.8\left(v_{1 / 2} \sim 330 \mathrm{~Hz}\right)$.

${ }^{19} \mathrm{~F}$ NMR $\left(564 \mathrm{MHz}\right.$, dichloromethane- $\left.d_{2}, 299 \mathrm{~K}\right): \delta-127.2(\mathrm{~m}, 0),-137.6\left(\mathrm{~m}, o^{\prime}\right),-160.9\left(\mathrm{t},{ }^{3} J_{\mathrm{FF}}\right.$ $=20.2 \mathrm{~Hz}, p),-165.4\left(\mathrm{~m}, m^{\prime}\right),-166.1(\mathrm{~m}, \mathrm{~m})\left(\right.$ each $\left.1 \mathrm{~F}, \mathrm{C}_{6} \mathrm{~F}_{5}\right)\left[\Delta \delta^{19} \mathrm{~F}_{\mathrm{mp}}=4.5,5.1\right],-130.4(\mathrm{~m}, o)$, $-133.2\left(\mathrm{~m}, o^{\prime}\right),-162.6\left(\mathrm{t},{ }^{3} \mathrm{JFF}_{\mathrm{FF}}=20.4 \mathrm{~Hz}, p\right),-166.3\left(\mathrm{~m}, \mathrm{~m}^{\prime}\right),-168.2(\mathrm{~m}, \mathrm{~m})\left(\right.$ each $\left.1 \mathrm{~F}, \mathrm{C}_{6} \mathrm{~F}_{5}\right)\left[\Delta \delta^{19} \mathrm{~F}_{\mathrm{mp}}\right.$ $=3.7,5.5]$.

Minor component:

${ }^{31} \mathbf{P}\left\{{ }^{1} \mathrm{H}\right\}$ NMR $\left(243 \mathrm{MHz}\right.$, dichloromethane- $\left.d_{2}, 299 \mathrm{~K}\right): \delta 16.5\left(v_{1 / 2} \sim 30 \mathrm{~Hz}\right)$.

${ }^{11} \mathbf{B}\left\{{ }^{1} \mathrm{H}\right\}$ NMR $\left(192 \mathrm{MHz}\right.$, dichloromethane- $\left.d_{2}, 299 \mathrm{~K}\right): \delta 0.8\left(v_{1 / 2} \sim 330 \mathrm{~Hz}\right)$.

${ }^{19}$ F NMR (564 MHz, dichloromethane- $\left.d_{2}, 299 \mathrm{~K}\right): \delta$-127.0, -129.7, -132.3, -136.9 (each br, each $1 \mathrm{~F}, o$ ), -160.5, -162.9 (each br, each 1F, $p$ ), not resolved $(4 \mathrm{~F}, m)\left(\mathrm{C}_{6} \mathrm{~F}_{5}\right)$. 

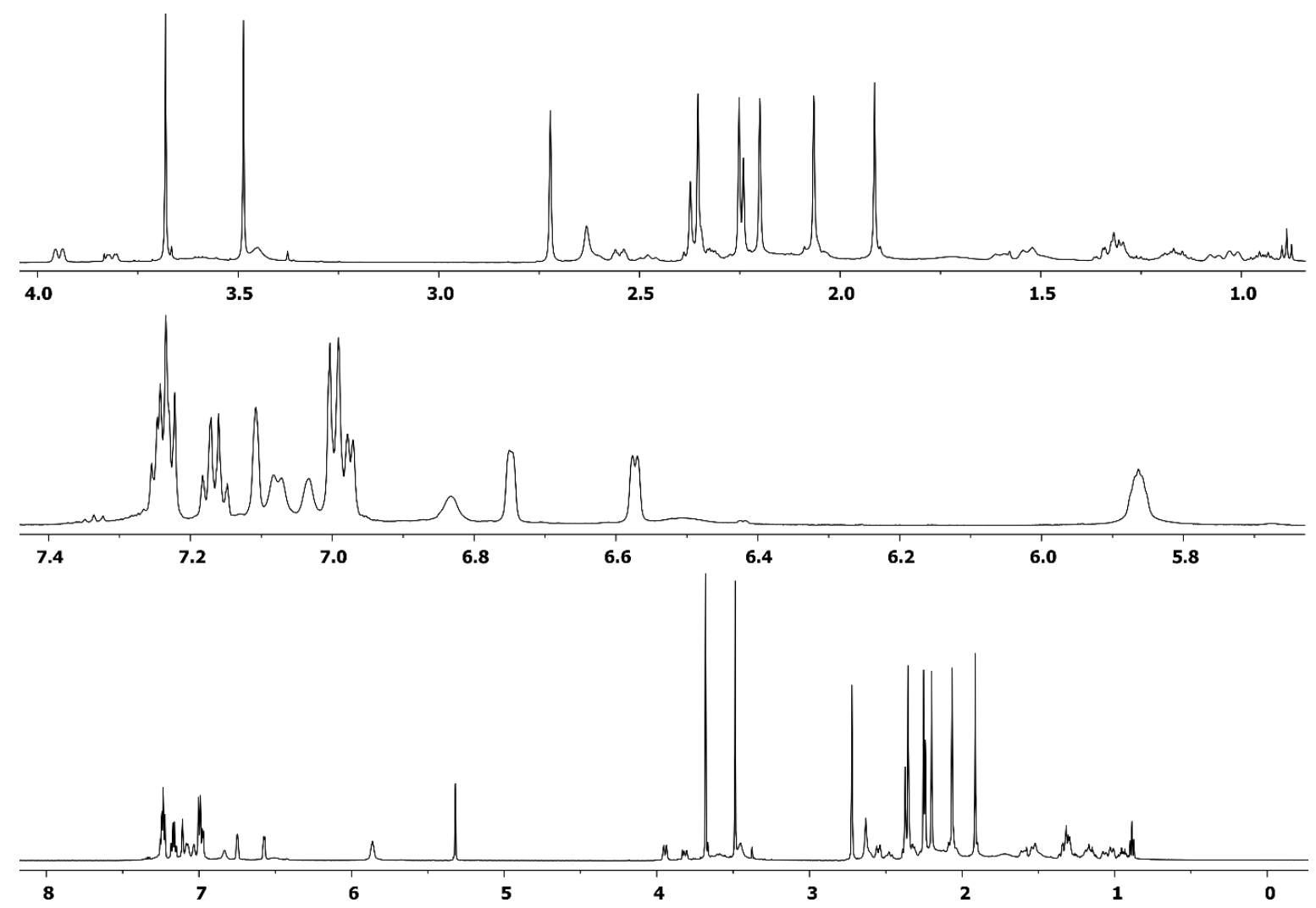

Figure S52: ${ }^{1} \mathbf{H}$ NMR (600 MHz, dichloromethane- $d_{2}$, $\left.299 \mathrm{~K}\right)$ spectrum of compound 17

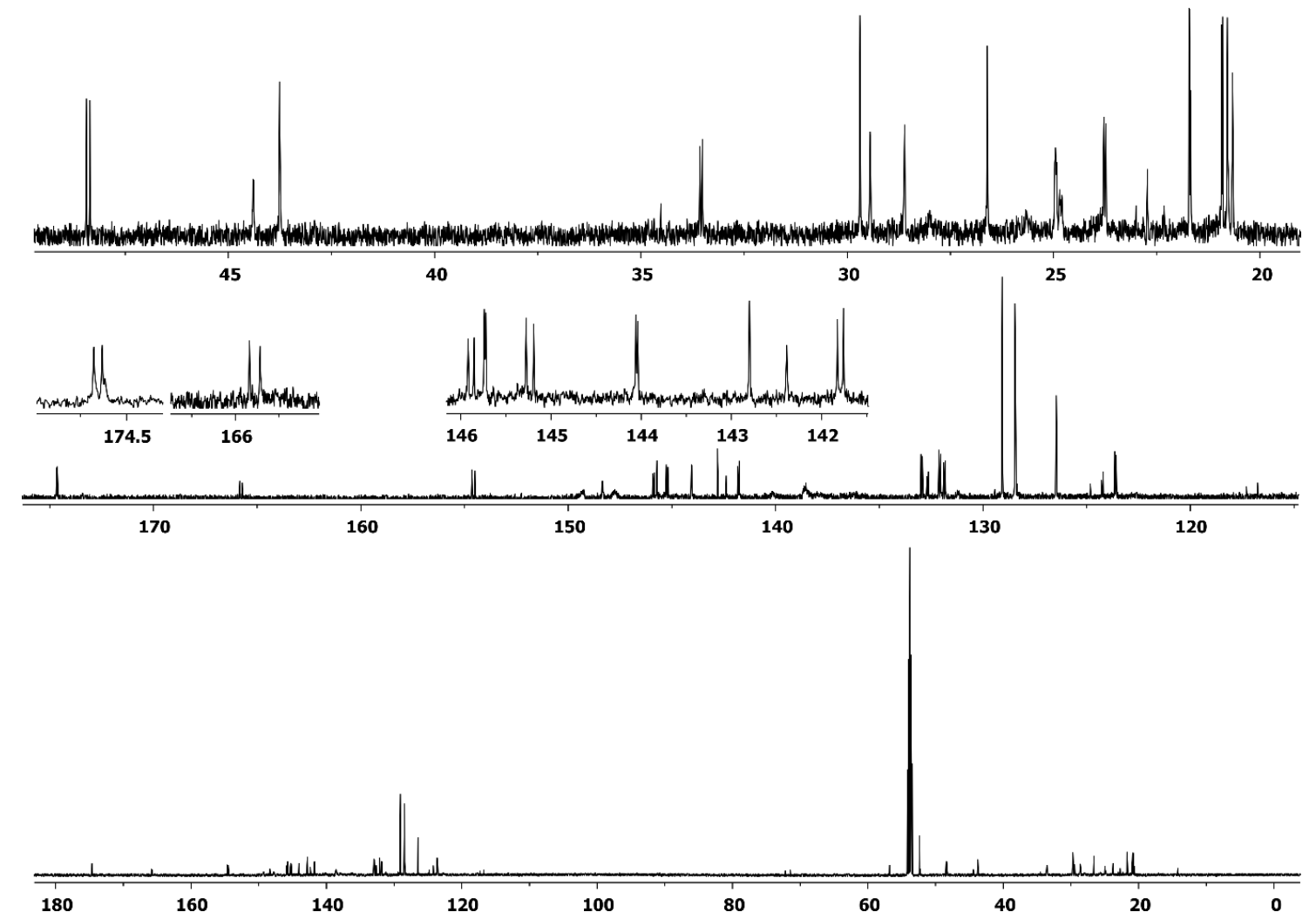

Figure S53: ${ }^{13} \mathbf{C}\left\{{ }^{1} \mathbf{H}\right\}$ NMR (151 MHz, dichloromethane- $\left.d_{2}, 299 \mathrm{~K}\right)$ spectrum of compound 17 


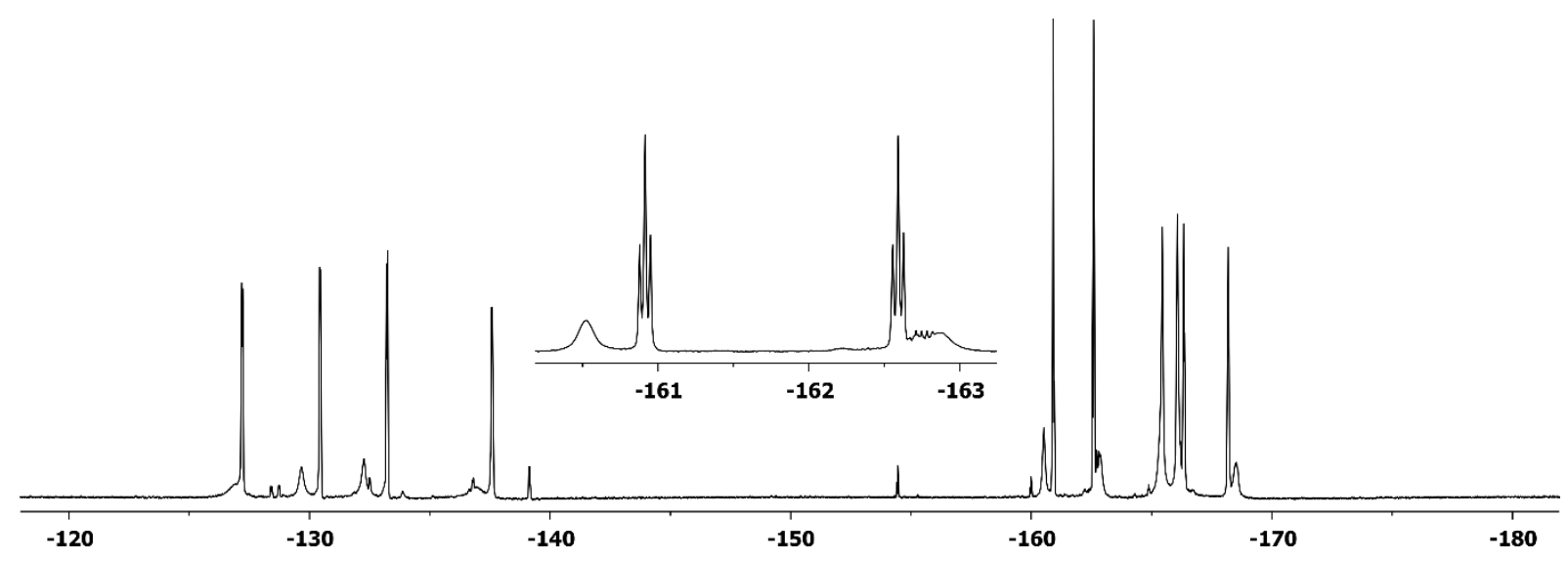

Figure S54: ${ }^{19} \mathrm{~F}$ NMR (564 MHz, dichloromethane- $d_{2}, 299 \mathrm{~K}$ ) spectrum of compound 17

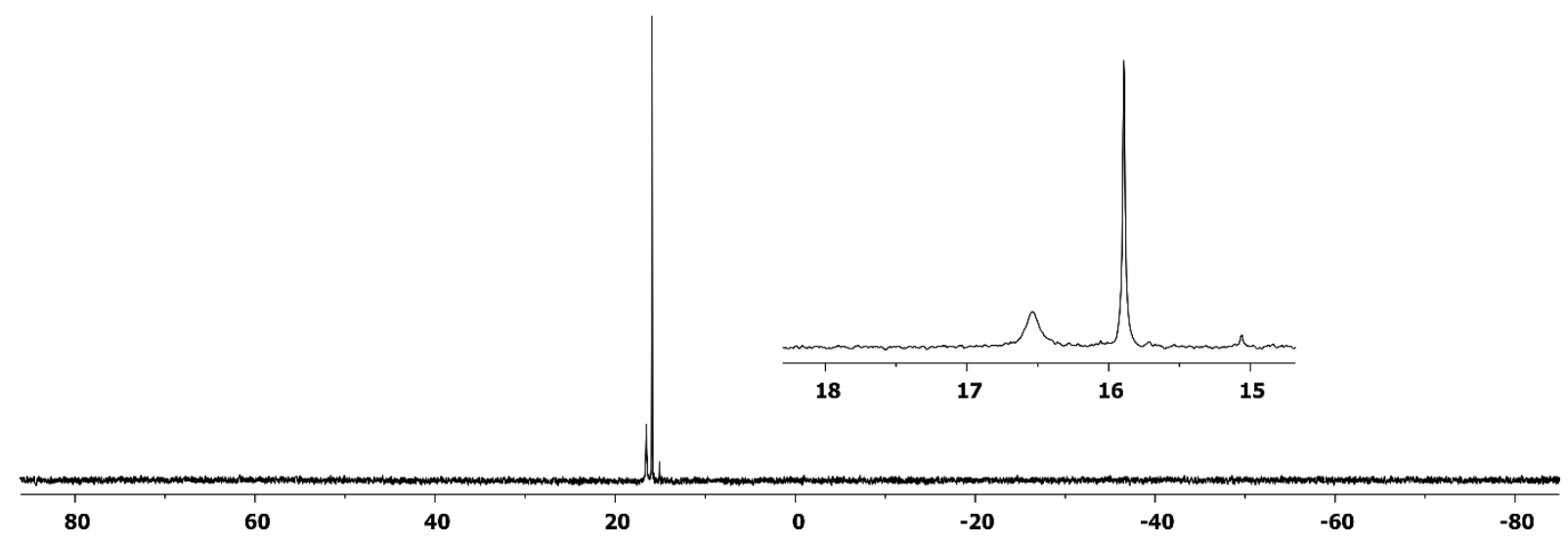

Figure S55: ${ }^{31} \mathrm{P}\left\{{ }^{1} \mathrm{H}\right\}$ NMR $\left(243 \mathrm{MHz}\right.$, dichloromethane- $\left.d_{2}, 299 \mathrm{~K}\right)$ spectrum of compound 17

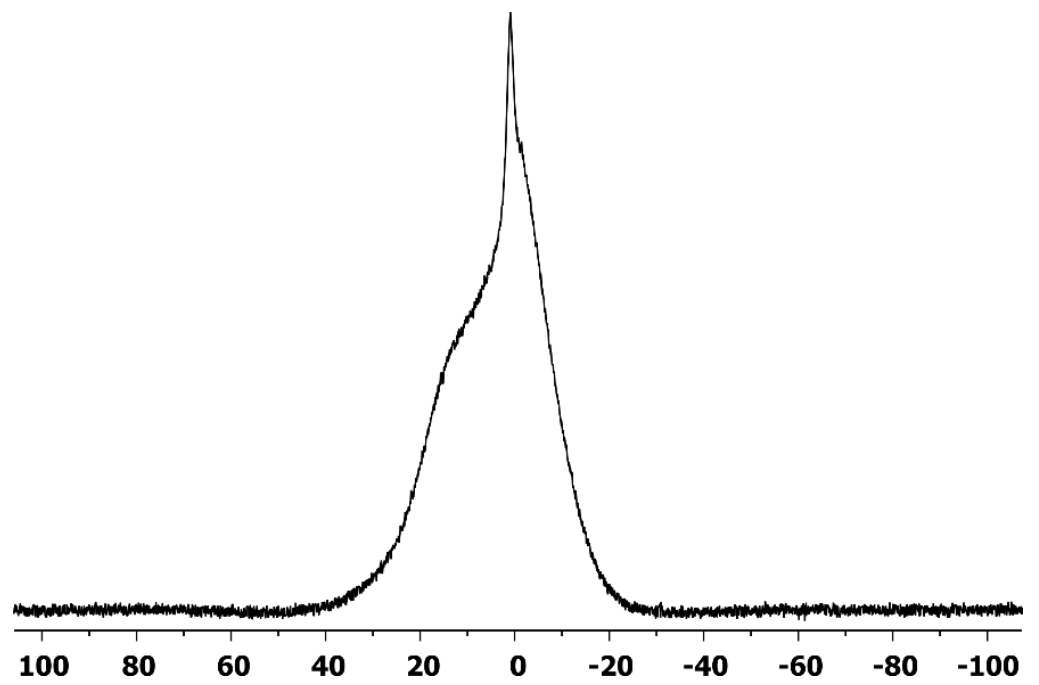

Figure S56: ${ }^{11} \mathbf{B}\left\{{ }^{1} \mathbf{H}\right\}$ NMR (192 MHz, dichloromethane-d2, $\left.299 \mathrm{~K}\right)$ spectrum of compound 17

Crystals suitable for the X-ray crystal structure analysis were obtained from a dichloromethane solution of compound $\mathbf{1 7}$ at room temperature:

X-ray crystal structure analysis of compound 17: A colorless prism-like specimen of $\mathrm{C}_{52} \mathrm{H}_{44} \mathrm{BF}_{10} \mathrm{O}_{4} \mathrm{P}$, approximate dimensions $0.110 \mathrm{~mm} \times 0.137 \mathrm{~mm} \times 0.206 \mathrm{~mm}$, was used for the $\mathrm{X}$-ray crystallographic analysis. The X-ray intensity data were measured. A total of 748 frames 
were collected. The total exposure time was 8.31 hours. The frames were integrated with the Bruker SAINT software package using a narrow-frame algorithm. The integration of the data using a monoclinic unit cell yielded a total of 8271 reflections to a maximum $\theta$ angle of $25.03^{\circ}$ (0.84 $\AA$ resolution), of which 8271 were independent (average redundancy 1.000, completeness $\left.=99.8 \%, R_{\text {int }}=8.35 \%, R_{\text {sig }}=3.22 \%\right)$ and $6534(79.00 \%)$ were greater than $2 \sigma\left(F^{2}\right)$. The final cell constants of $\underline{a}=14.9814(7) \AA$, $\underline{b}=16.7671(7) \AA \underline{c}=19.0481(8) \AA, \beta=$ $100.8210(10)^{\circ}$, volume $=4699.7(4) \AA^{3}$, are based upon the refinement of the XYZ-centroids of 9841 reflections above $20 \sigma(\mathrm{I})$ with $4.858^{\circ}<2 \theta<54.47^{\circ}$. Data were corrected for absorption effects using the multi-scan method (SADABS). The ratio of minimum to maximum apparent transmission was 0.959 . The calculated minimum and maximum transmission coefficients (based on crystal size) are 0.9710 and 0.9840 . The structure was solved and refined using the Bruker SHELXTL Software Package, using the space group P $121 / n 1$, with $Z=4$ for the formula unit, $\mathrm{C}_{52} \mathrm{H}_{44} \mathrm{BF}_{10} \mathrm{O}_{4} \mathrm{P}$. The final anisotropic full-matrix least-squares refinement on $\mathrm{F}^{2}$ with 621 variables converged at $R 1=4.87 \%$, for the observed data and $w R 2=11.16 \%$ for all data. The goodness-of-fit was 1.066. The largest peak in the final difference electron density synthesis was $0.378 \mathrm{e}^{-} / \AA^{3}$ and the largest hole was $-0.345 \mathrm{e}^{-} / \AA^{3}$ with an RMS deviation of 0.054 $\mathrm{e}^{-} / \AA^{3}$. On the basis of the final model, the calculated density was $1.363 \mathrm{~g} / \mathrm{cm}^{3}$ and $F(000)$, $1992 \mathrm{e}^{-}$.

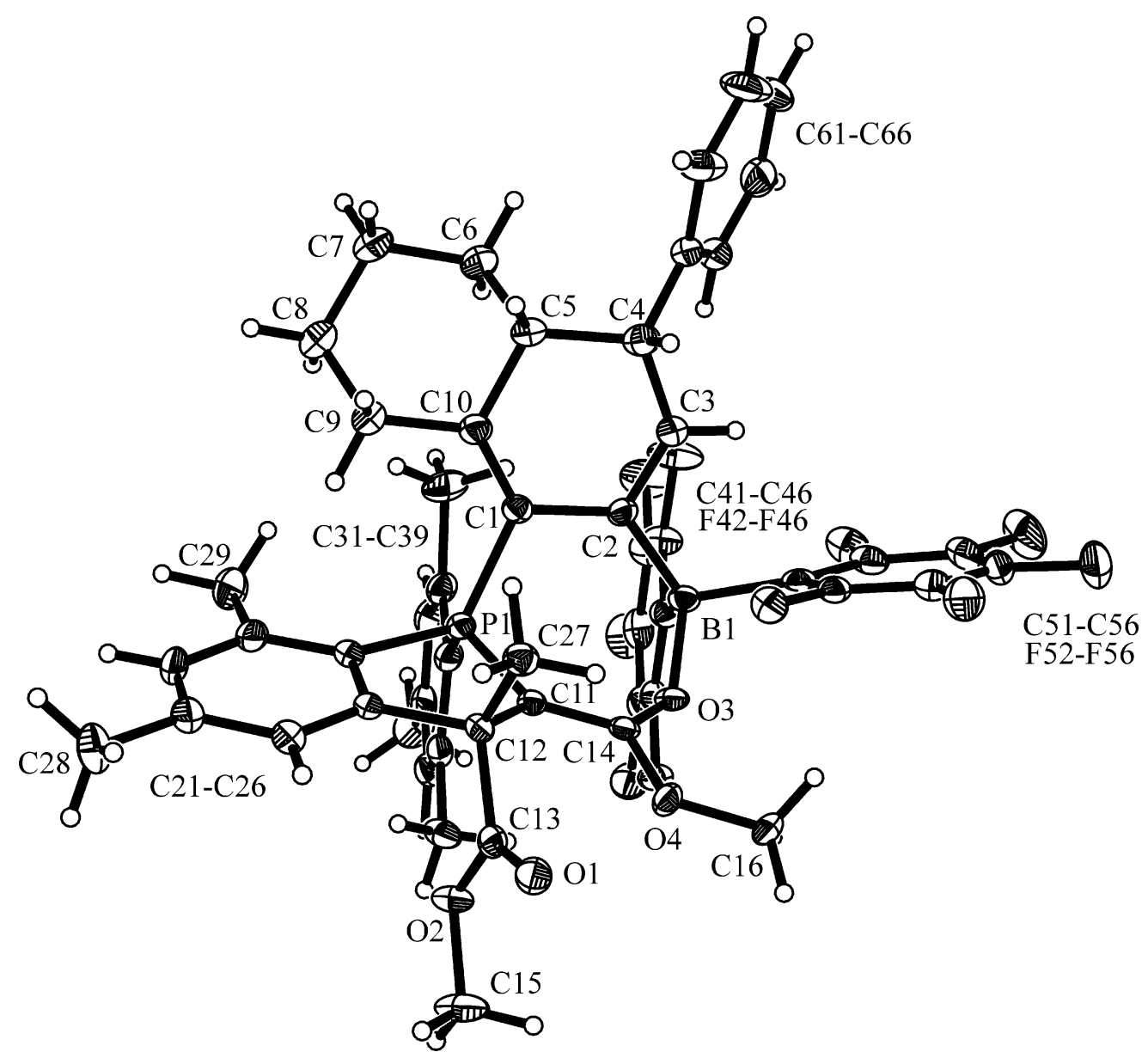

Figure S57: X-ray crystal structure of compound 17 (thermal ellipsoids are shown at the 50\% probability level) 


\section{Synthesis of compound 18}
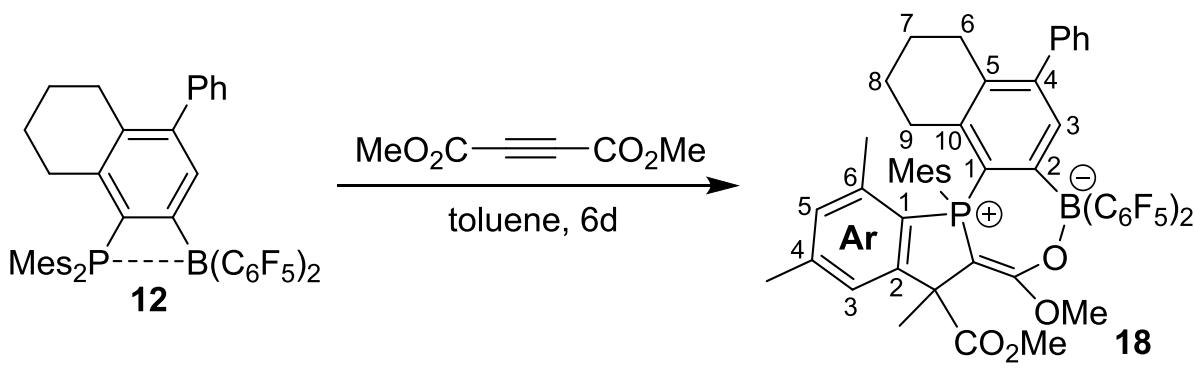

Scheme S10

A solution of dimethyl acetylenedicarboxylate $(18.0 \mu \mathrm{L}, 20.8 \mathrm{mg}, 146.2 \mu \mathrm{mol}, 1.0 \mathrm{eq})$ in toluene $(1 \mathrm{~mL})$ was added to a solution of compound $12(120.0 \mathrm{mg}, 146.2 \mu \mathrm{mol}, 1.0 \mathrm{eq}) \mathrm{in}$ toluene $(3 \mathrm{~mL})$ and the reaction mixture was stirred at $100^{\circ} \mathrm{C}$ for 6 days. Then all volatiles were removed in vacuo and the formed sticky residue was dissolved in pentane $(5 \mathrm{~mL})$, which was subsequently removed in vacuo. After adding pentane $(3 \mathrm{~mL})$ to the obtained sticky solid the resulting suspension was filtered via cannula (Whatman glass fiber filter). Then the remaining solid was washed with pentane $(5 \times 3 \mathrm{~mL})$ and dried in vacuo to give compound 18 as a pale orange solid ( $87.7 \mathrm{mg}, 91.1 \mu \mathrm{mol}, 62 \%)$.

IR $(\mathrm{KBr}): \tilde{v}\left[\mathrm{~cm}^{-1}\right]=3473(\mathrm{w}), 3027(\mathrm{w}), 2938(\mathrm{~m}), 2864(\mathrm{w}), 2668(\mathrm{w}), 2540(\mathrm{w}), 2293(\mathrm{w}), 2092$ $(\mathrm{w}), 1744(\mathrm{~s}), 1613$ (s), $1561(\mathrm{w}), 1515(\mathrm{~s}), 1450$ (s), $1377(\mathrm{w}), 1338(\mathrm{~m}), 1244(\mathrm{~m}), 1024(\mathrm{w})$, $1150(w), 1088$ (s), 1030 (w), $974(\mathrm{~s}), 926(\mathrm{w}), 875(\mathrm{w}), 854(\mathrm{~m}), 814(\mathrm{w}), 778(\mathrm{~m}), 764(\mathrm{w})$, $747(m), 710(w), 690(m), 662(w), 641(w), 604(w), 563(w), 521(w), 459(w), 444(w), 419$ (w).

M.p. $268^{\circ} \mathrm{C}$.

Anal. Calc. for $\mathrm{C}_{52} \mathrm{H}_{42} \mathrm{BF}_{10} \mathrm{O}_{4} \mathrm{P}: \mathrm{C}: 64.88 ; \mathrm{H}: 4.40$. Found: $\mathrm{C}: 64.91 ; \mathrm{H}: 4.59$.

$[\mathrm{Ar}=$ 4,6-dimethylphenylene]

${ }^{1} \mathrm{H}$ NMR (500 MHz, dichloromethane- $\left.d_{2}, 299 \mathrm{~K}\right): \delta 7.34(\mathrm{~m}, 2 \mathrm{H}, m-\mathrm{Ph}), 7.27(\mathrm{~m}, 1 \mathrm{H}, p-\mathrm{Ph})$, $7.16(\mathrm{~m}, 2 \mathrm{H}, \mathrm{o}-\mathrm{Ph}), 7.13(\mathrm{dd}, J=8.1 \mathrm{~Hz}, J=5.3 \mathrm{~Hz}, 1 \mathrm{H}, 3-\mathrm{CH}), 7.04\left(\mathrm{~m}, 1 \mathrm{H}, 3-\mathrm{CH}^{\mathrm{Ar}}\right), 7.03$ $\left(\mathrm{m}, 1 \mathrm{H}, 5-\mathrm{CH}^{\mathrm{Ar}}\right), 6.84\left(\mathrm{dm},{ }^{4} \mathrm{~J}_{\mathrm{PH}}=3.6 \mathrm{~Hz}, 1 \mathrm{H}, m-\mathrm{Mes}\right), 6.42\left(\mathrm{dm},{ }^{4} J_{\mathrm{PH}}=4.6 \mathrm{~Hz}, 1 \mathrm{H}, m^{\prime}-\mathrm{Mes}\right)$, $3.66\left(\mathrm{~s}, 3 \mathrm{H} . \mathrm{OMe}^{\mathrm{C}=\mathrm{O}} \text { ), } 3.38 \text { (s, 3H, OMe), 2.74/2.28 (each m, each } 1 \mathrm{H}, 9-\mathrm{CH}_{2}\right)^{\mathrm{t}}, 2.55 / 2.50$ (each m, each $\left.1 \mathrm{H}, 6-\mathrm{CH}_{2}\right)^{\mathrm{t}}, 2.39\left(\mathrm{~d}, J=0.8 \mathrm{~Hz}, 3 \mathrm{H}, 4-\mathrm{CH}_{3}{ }^{\mathrm{Ar}}\right.$ ), $2.34\left(\mathrm{~s}, 3 \mathrm{H}, 0-\mathrm{CH}_{3}{ }^{\mathrm{Mes}}\right)^{\mathrm{t}}, 2.20$ (s, $3 \mathrm{H}, p-\mathrm{CH}_{3}{ }^{\mathrm{Mes}}$ ), 2.09 (s, 3H, 6- $\mathrm{CH}_{3}{ }^{\mathrm{Ar}}$ ), 1.90 (s, 3H, o'- $\left.\mathrm{CH}_{3}{ }^{\mathrm{Mes}}\right)^{\mathrm{t}}, 1.59 / 1.43$ (each m, each 1H, 7$\left.\mathrm{CH}_{2}\right)^{\mathrm{t}}, 1.58\left(\mathrm{~s}, 3 \mathrm{H}, \mathrm{CH}_{3}\right), 1.51\left(\mathrm{~m}, 2 \mathrm{H}, 8-\mathrm{CH}_{2}\right)^{\mathrm{t}}$. [ ${ }^{\mathrm{t}}$ tentatively assigned]

${ }^{13} \mathrm{C}\left\{{ }^{1} \mathrm{H}\right\}$ NMR $\left(126 \mathrm{MHz}\right.$, dichloromethane- $\left.d_{2}, 299 \mathrm{~K}\right): \delta 174.6\left(\mathrm{~d},{ }^{3} J_{\mathrm{PC}}=8.6 \mathrm{~Hz}, \mathrm{O}=\mathrm{C}\right), 162.6$ $\left(\mathrm{d},{ }^{2} J_{\mathrm{PC}}=16.3 \mathrm{~Hz}, \mathrm{O}_{2} \mathrm{C}=\right), 154.9\left(\mathrm{~d},{ }^{2} \mathrm{JPC}_{\mathrm{PC}}=22.4 \mathrm{~Hz}, 2-\mathrm{C}^{\mathrm{Ar}}\right), 146.0\left(\mathrm{~d},{ }^{2} \mathrm{~J}_{\mathrm{PC}}=9.8 \mathrm{~Hz}, 0-\mathrm{Mes}\right)^{\mathrm{t}}$, $145.7\left(\mathrm{~d},{ }^{4} \mathrm{~J}_{\mathrm{PC}}=2.6 \mathrm{~Hz}, 4-\mathrm{C}^{\mathrm{Ar}}\right), 145.4\left(\mathrm{~d},{ }^{2} \mathrm{~J}_{\mathrm{PC}}=12.9 \mathrm{~Hz}, o^{\prime}-\mathrm{Mes}\right){ }^{\mathrm{t}}, 144.3\left(\mathrm{~d},{ }^{4} J_{\mathrm{PC}}=3.0 \mathrm{~Hz}, p-\right.$ 
Mes), 143.9 (dd, $J=3.4 \mathrm{~Hz}, J=1.1 \mathrm{~Hz}, 4-\mathrm{C}$ ), 142.1 (d, $J=1.2 \mathrm{~Hz}, i-\mathrm{Ph}), 142.0\left(\mathrm{~d},{ }^{2} \mathrm{JPC}=\right.$ $9.9 \mathrm{~Hz}, 6-\mathrm{C}^{\mathrm{Ar}}$ ), 139.8 (dd, $\left.J=18.5 \mathrm{~Hz}, J=9.8 \mathrm{~Hz}, 3-\mathrm{CH}\right), 135.8\left(\mathrm{~d},{ }^{1} J_{\mathrm{PC}}=80.8 \mathrm{~Hz}, 1-\mathrm{C}\right)$, $134.8\left(\mathrm{~d},{ }^{2} J_{\mathrm{PC}}=11.3 \mathrm{~Hz}, 10-\mathrm{C}\right)^{\mathrm{t}}, 134.6\left(\mathrm{~d},{ }^{3} \mathrm{~J}_{\mathrm{PC}}=11.5 \mathrm{~Hz}, 5-\mathrm{C}\right) \mathrm{t}, 133.2\left(\mathrm{~d},{ }^{3} \mathrm{JPC}_{\mathrm{PC}}=10.2 \mathrm{~Hz}, 5-\right.$ $\mathrm{CH}^{\mathrm{Ar}}$ ), 131.7 (d, $\left.{ }^{3} \mathrm{JPC}_{\mathrm{PC}}=6.2 \mathrm{~Hz}, m^{\prime}-\mathrm{Mes}\right), 131.6$ (d, $\left.{ }^{3} \mathrm{JPC}_{\mathrm{PC}}=5.1 \mathrm{~Hz}, m-\mathrm{Mes}\right), 129.4$ (o-Ph), 128.4 $(m-\mathrm{Ph}), 127.2(p-\mathrm{Ph}), 124.6\left(\mathrm{~d},{ }^{1} \mathrm{JPC}_{\mathrm{PC}}=84.2 \mathrm{~Hz}, 1-\mathrm{C}^{\mathrm{Ar}}\right), 123.5\left(\mathrm{~d},{ }^{3} \mathrm{~J}_{\mathrm{PC}}=10.0 \mathrm{~Hz}, 3-\mathrm{CH}^{\mathrm{Ar}}\right)$, $116.3\left(\mathrm{~d},{ }^{1} J_{\mathrm{PC}}=82.8 \mathrm{~Hz}, i-\mathrm{Mes}\right), 77.8\left(\mathrm{~d},{ }^{1} J_{\mathrm{PC}}=115.4 \mathrm{~Hz}, \mathrm{PC}=\right), 55.7$ (d, ${ }^{2} J_{\mathrm{PC}}=15.2 \mathrm{~Hz}$, $\mathrm{CMe}), 53.7\left(\mathrm{~d},{ }^{4} J_{\mathrm{PC}}=1.2 \mathrm{~Hz}, \mathrm{OMe}\right), 52.4\left(\mathrm{OMe}^{\mathrm{C}=0}\right), 29.4\left(\mathrm{~d},{ }^{3} J_{\mathrm{PC}}=7.1 \mathrm{~Hz}, 9-\mathrm{CH}_{2}\right)^{\mathrm{t}}, 28.7(\mathrm{~d}$, $\left.{ }^{4} J_{\mathrm{PC}}=1.6 \mathrm{~Hz}, 6-\mathrm{CH}_{2}\right)^{\mathrm{t}}, 28.2\left(\mathrm{CH}_{3}\right), 24.2\left(\mathrm{dd}, J=5.8 \mathrm{~Hz}, J=3.3 \mathrm{~Hz} o^{\prime}-\mathrm{Mes}\right)^{\mathrm{t}}, 23.9$ (dd, $J=$ $7.0 \mathrm{~Hz}, J=1.5 \mathrm{~Hz}, o-\mathrm{Mes})^{\mathrm{t}}, 23.0\left(8-\mathrm{CH}_{2}\right)^{\mathrm{t}}, 22.3\left(7-\mathrm{CH}_{2}\right)^{\mathrm{t}}, 21.7\left(\mathrm{~d}, J=1.3 \mathrm{~Hz}, 4-\mathrm{CH}_{3}{ }^{\mathrm{Ar}}\right), 20.9$ $\left(\mathrm{d},{ }^{3} \mathrm{JCC}=3.4 \mathrm{~Hz}, 6-\mathrm{CH}_{3}{ }^{\mathrm{Ar}}\right), 20.7$ (p-Mes), n.o. (2-C). [ $\mathrm{C}_{6} \mathrm{~F}_{5}$ not listed; ${ }^{\mathrm{t}}$ tentatively assigned] ${ }^{31} \mathbf{P}\left\{{ }^{1} \mathrm{H}\right\}$ NMR $\left(202 \mathrm{MHz}\right.$, dichloromethane- $\left.d_{2}, 213 \mathrm{~K}\right): \delta 15.1\left(v_{1 / 2} \sim 5 \mathrm{~Hz}\right)$.

${ }^{11} \mathbf{B}\left\{{ }^{1} \mathrm{H}\right\}$ NMR $\left(160 \mathrm{MHz}\right.$, dichloromethane- $\left.d_{2}, 299 \mathrm{~K}\right): \delta 1.1\left(v_{1 / 2} \sim 350 \mathrm{~Hz}\right)$.

${ }^{19} \mathrm{~F}$ NMR $\left(470 \mathrm{MHz}\right.$, dichloromethane- $\left.d_{2}, 299 \mathrm{~K}\right): \delta-128.5(\mathrm{~m}, \mathrm{o}),-136.8\left(\mathrm{~m}, \mathrm{o}^{\prime}\right),-160.0\left(\mathrm{t},{ }^{3} J_{\mathrm{FF}}\right.$ $=20.3 \mathrm{~Hz}, p),-164.9\left(\mathrm{~m}, m^{\prime}\right),-165.5(\mathrm{~m}, \mathrm{~m})\left(\right.$ each $\left.1 \mathrm{~F}, \mathrm{C}_{6} \mathrm{~F}_{5}\right)\left[\Delta \delta^{19} \mathrm{~F}_{\mathrm{mp}}=4.9,5.5\right],-128.8(\mathrm{~m}, o)$, $-132.6\left(\mathrm{~m}, o^{\prime}\right),-162.8\left(\mathrm{t},{ }^{3} J_{\mathrm{FF}}=20.4 \mathrm{~Hz}, p\right),-166.2\left(\mathrm{~m}, m^{\prime}\right),-168.6(\mathrm{~m}, m)\left(\right.$ each $\left.1 \mathrm{~F}, \mathrm{C}_{6} \mathrm{~F}_{5}\right)\left[\Delta \delta^{19} \mathrm{~F}_{\mathrm{mp}}\right.$ $=3.4,5.8]$.
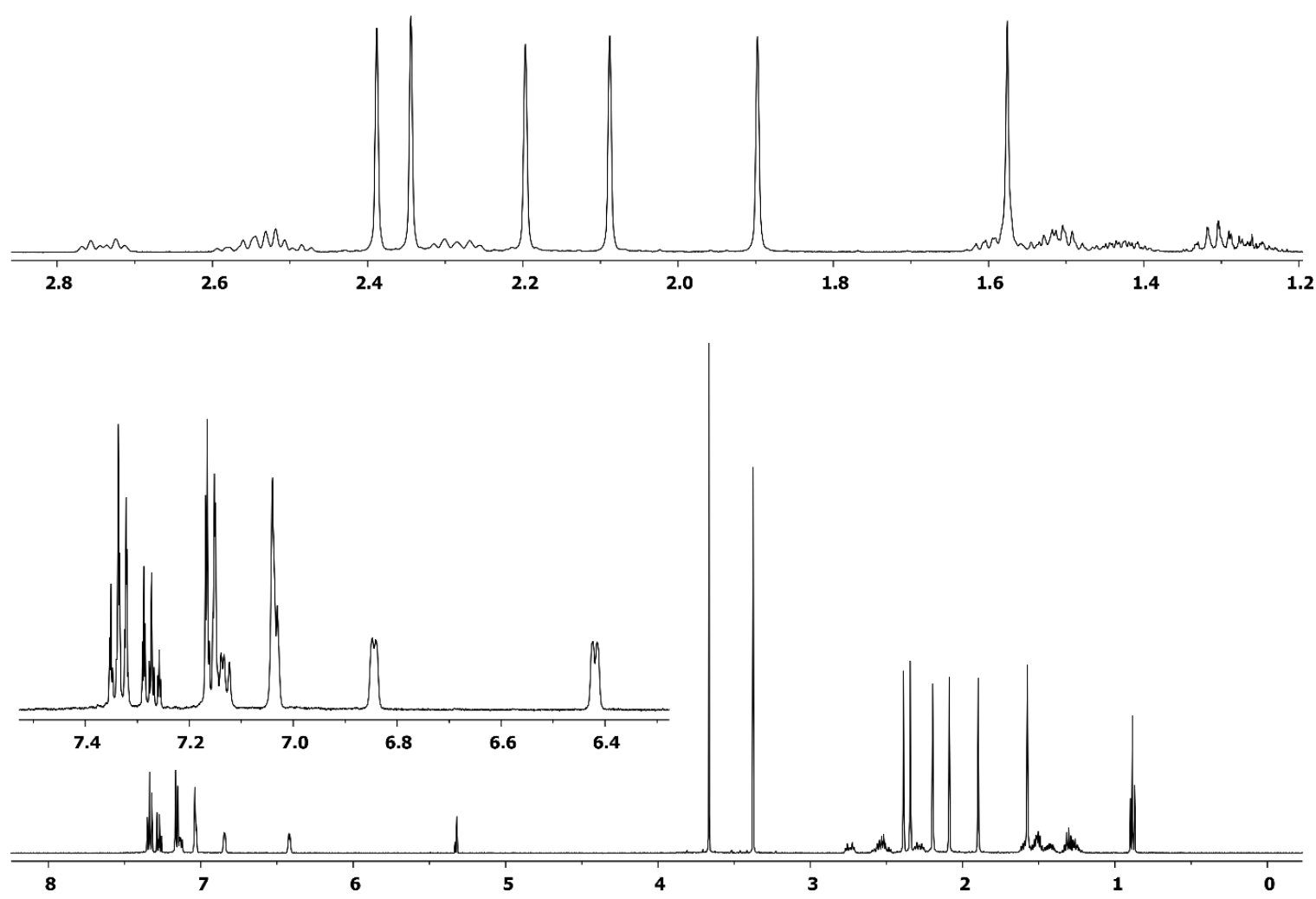

Figure S58: ${ }^{1} \mathbf{H}$ NMR $\left(500 \mathrm{MHz}\right.$, dichloromethane- $\left.d_{2}, 299 \mathrm{~K}\right)$ spectrum of compound 18 


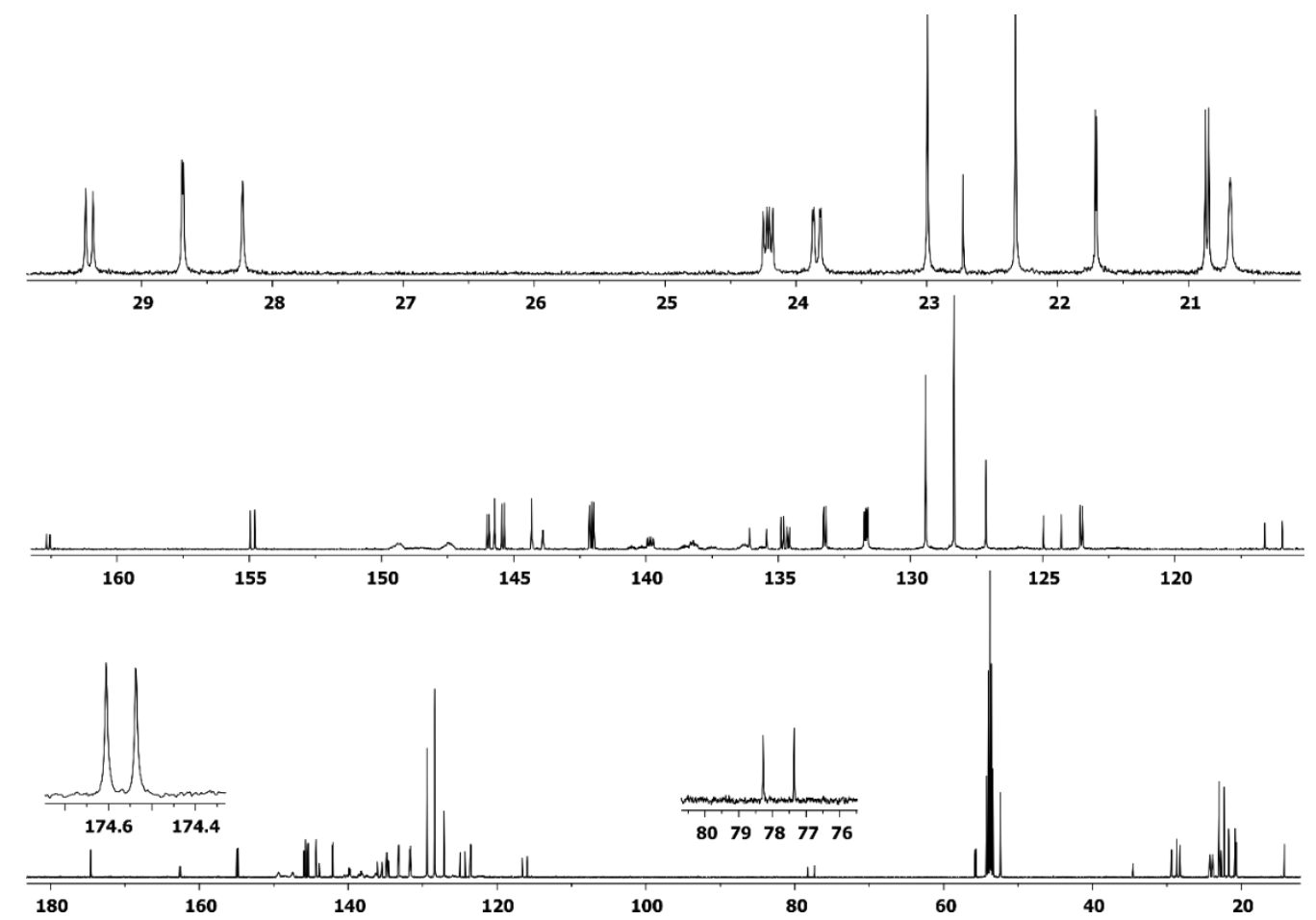

Figure S59: ${ }^{13} \mathrm{C}\left\{{ }^{1} \mathrm{H}\right\}$ NMR (126 MHz, dichloromethane- $\left.d_{2}, 299 \mathrm{~K}\right)$ spectrum of compound 18

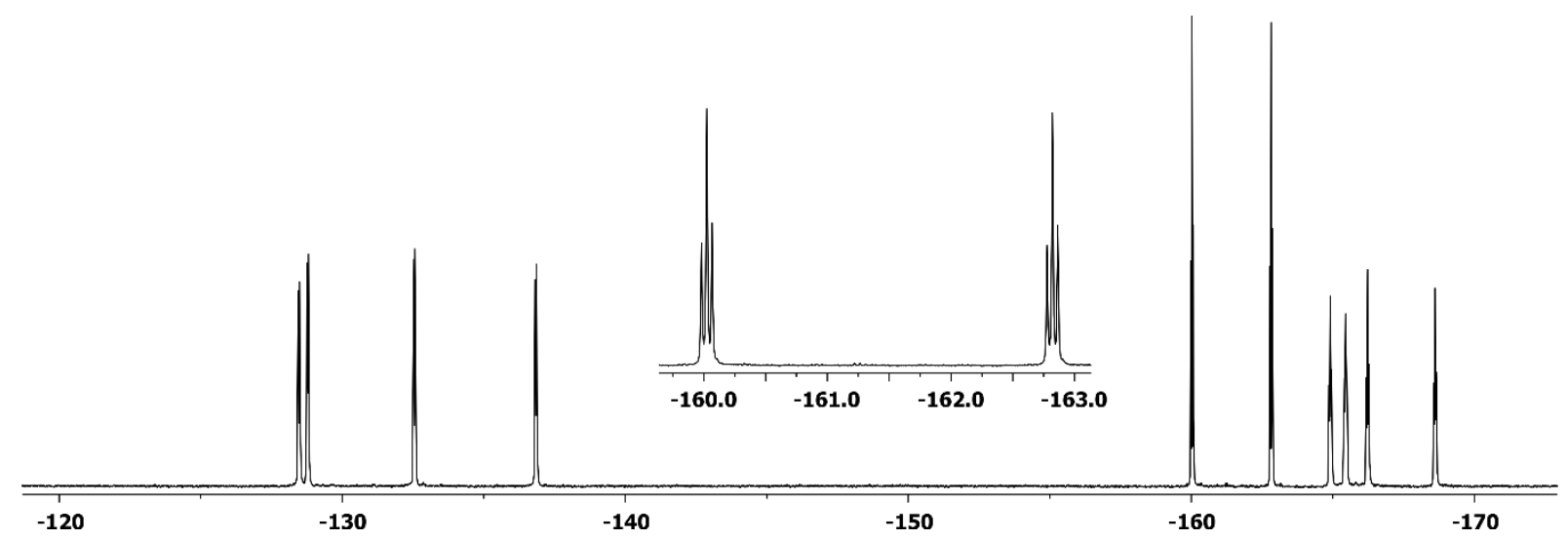

Figure S60: ${ }^{19} \mathrm{~F}$ NMR (470 MHz, dichloromethane- $\left.d_{2}, 299 \mathrm{~K}\right)$ spectrum of compound 18

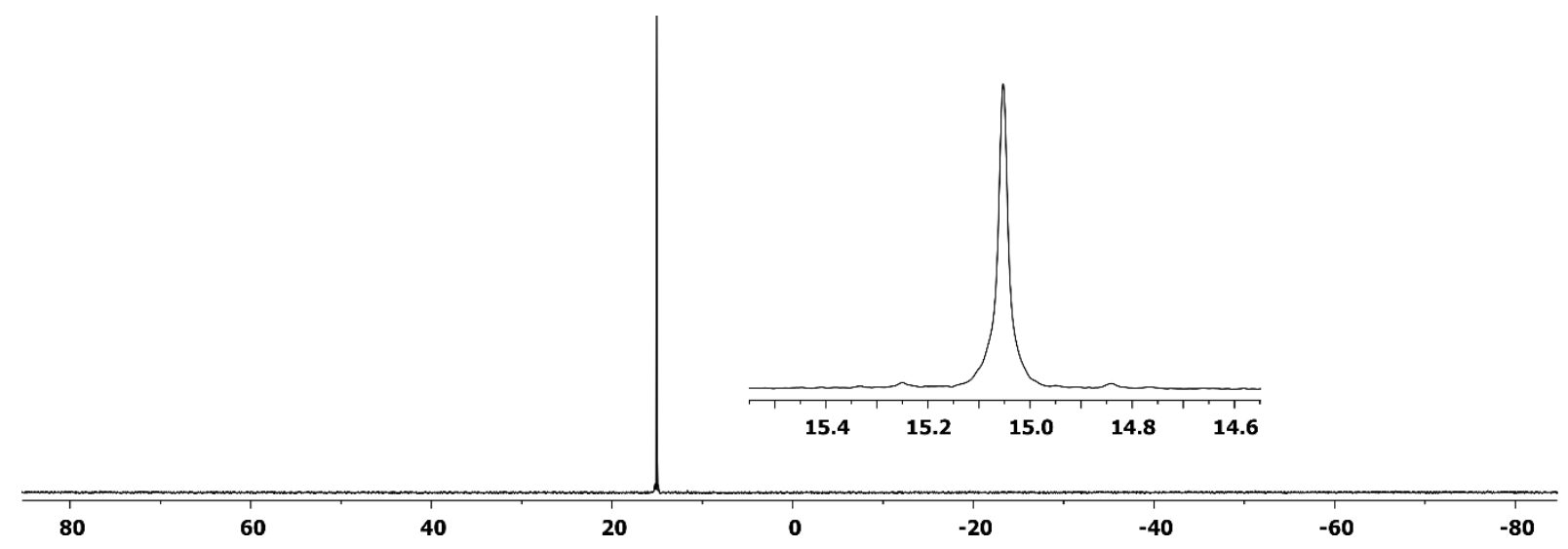

Figure S61: ${ }^{31} \mathbf{P}\left\{{ }^{1} \mathbf{H}\right\}$ NMR $\left(202 \mathrm{MHz}\right.$, dichloromethane- $\left.d_{2}, 299 \mathrm{~K}\right)$ spectrum of compound 18 


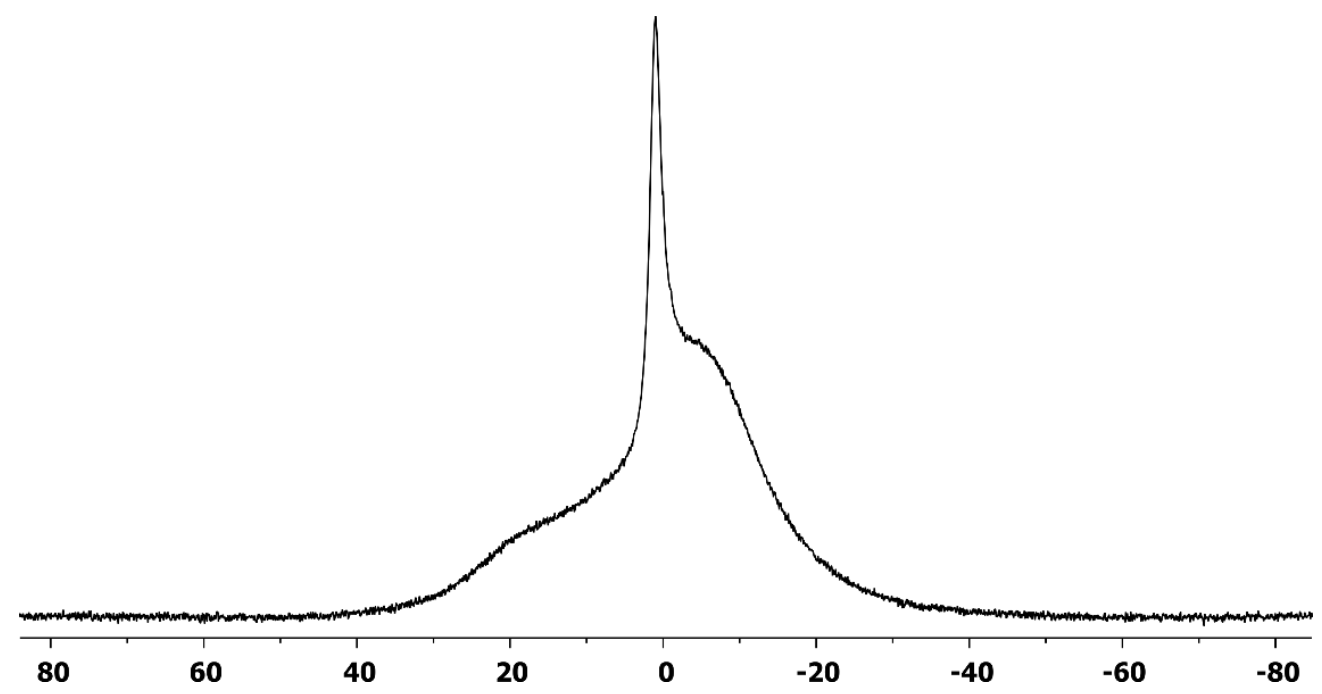

Figure S62: ${ }^{11} \mathbf{B}\left\{{ }^{1} \mathbf{H}\right\}$ NMR (160 MHz, dichloromethane- $\left.d_{2}, 299 \mathrm{~K}\right)$ spectrum of compound 18

Crystals suitable for the X-ray crystal structure analysis were obtained from a dichloromethane solution of compound 18 at $-35^{\circ} \mathrm{C}$ :

X-ray crystal structure analysis of compound 18: A colorless plate-like specimen of $\mathrm{C}_{52} \mathrm{H}_{42} \mathrm{BF}_{10} \mathrm{O}_{4} \mathrm{P}$, approximate dimensions $0.020 \mathrm{~mm} \times 0.080 \mathrm{~mm} \times 0.220 \mathrm{~mm}$, was used for the $X$-ray crystallographic analysis. The X-ray intensity data were measured. A total of 1592 frames were collected. The total exposure time was 20.62 hours. The frames were integrated with the Bruker SAINT software package using a wide-frame algorithm. The integration of the data using a monoclinic unit cell yielded a total of 8235 reflections to a maximum $\theta$ angle of $66.59^{\circ}$ (0.84 $\AA$ resolution), of which 8235 were independent (average redundancy 1.000, completeness $\left.=99.8 \%, R_{\text {int }}=9.30 \%, R_{\text {sig }}=5.39 \%\right)$ and $6008(72.96 \%)$ were greater than

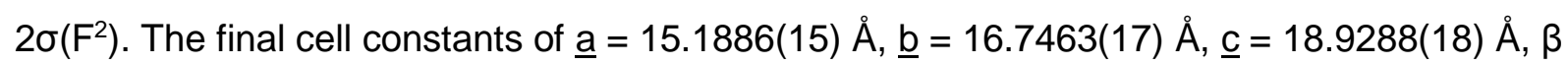
$=103.662(5)^{\circ}$, volume $=4678.4(8) \AA^{3}$, are based upon the refinement of the $X Y Z$-centroids of 9144 reflections above $20 \sigma(\mathrm{I})$ with $7.138^{\circ}<2 \theta<133.0^{\circ}$. Data were corrected for absorption effects using the multi-scan method (SADABS). The ratio of minimum to maximum apparent transmission was 0.839 . The calculated minimum and maximum transmission coefficients (based on crystal size) are 0.7680 and 0.9750 . The final anisotropic full-matrix least-squares refinement on $\mathrm{F}^{2}$ with 621 variables converged at $\mathrm{R} 1=4.81 \%$, for the observed data and $\mathrm{wR} 2$ $=12.26 \%$ for all data. The goodness-of-fit was 1.070 . The largest peak in the final difference electron density synthesis was $0.284 \mathrm{e}^{-/} / \AA^{3}$ and the largest hole was $-0.435 \mathrm{e}^{-} / \AA^{3}$ with an RMS deviation of $0.057 \mathrm{e}^{-} / \AA^{3}$. On the basis of the final model, the calculated density was $1.367 \mathrm{~g} / \mathrm{cm}^{3}$ and $F(000), 1984 \mathrm{e}^{-}$. 


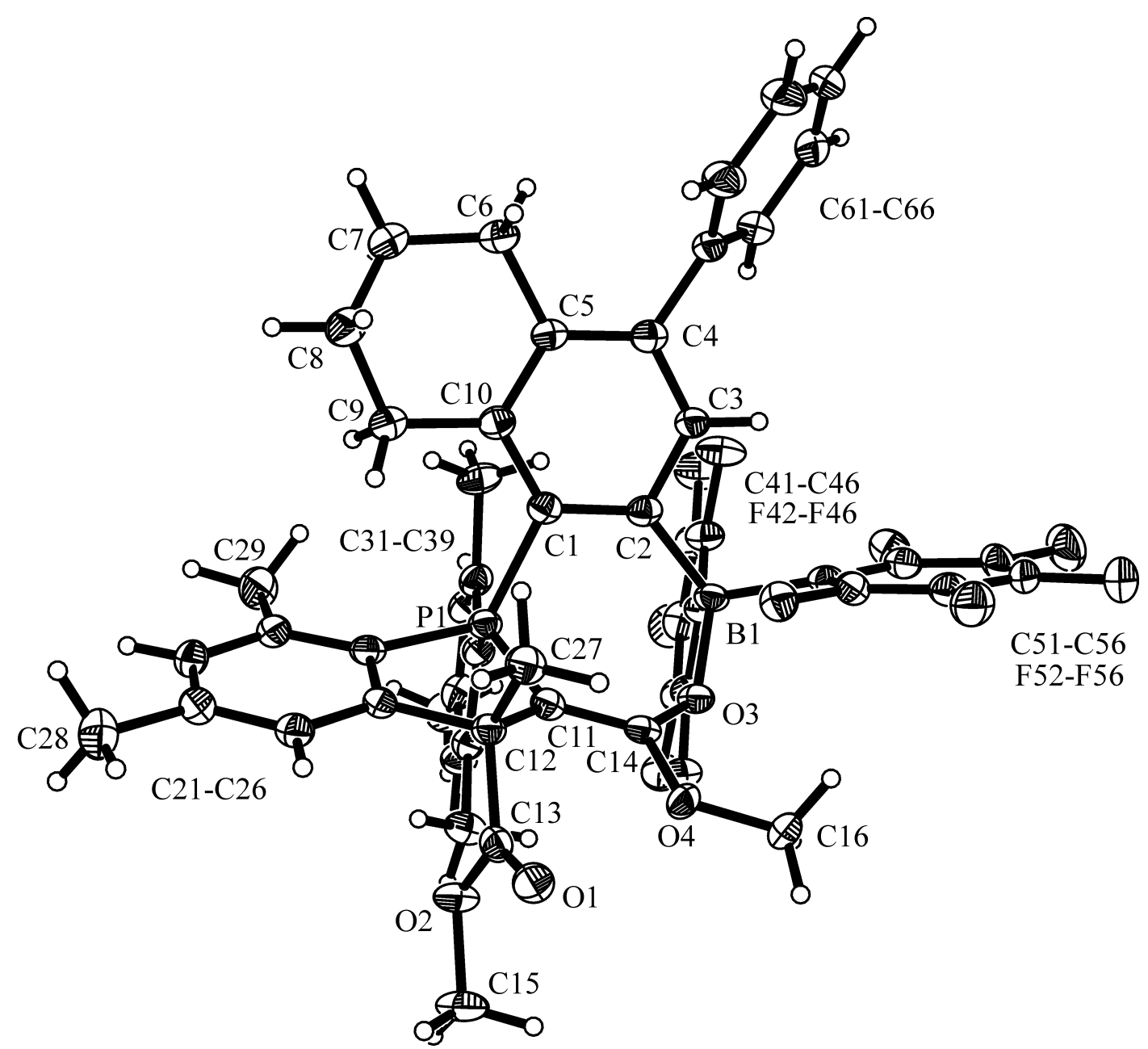

Figure S63: X-ray crystal structure of compound 18 (thermal ellipsoids are shown at the 50\% probability level)

\section{Synthesis of compound 19}

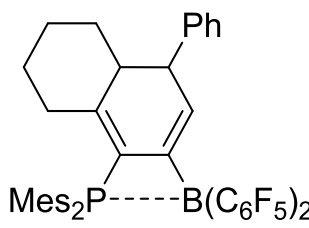

11

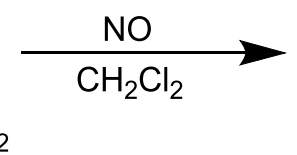

Scheme S11

A solution of compound 11 (200.0 mg, $243.1 \mu \mathrm{mol}, 1.0 \mathrm{eq})$ in $\mathrm{CH}_{2} \mathrm{Cl}_{2}(1 \mathrm{~mL})$ was degassed at $-78{ }^{\circ} \mathrm{C}$ and exposed to a nitric oxide atmosphere (1.5 bar). The solution turned dark turquoise upon stirring for $15 \mathrm{~min}$. Then all volatiles were removed in vacuo and pentane $(3 \mathrm{~mL})$ was added to the obtained residue. The suspension was filtrated via cannula (Whatman glass fiber filter) and the remaining solid was washed with pentane $(3 \times 2 \mathrm{~mL})$. After drying in vacuo compound 19 was obtained as a pale turquoise solid (166.4 mg, $195.2 \mu \mathrm{mol}, 80 \%)$.

IR (KBr): $\tilde{v}\left[\mathrm{~cm}^{-1}\right]=3515(\mathrm{w}), 3063(\mathrm{w}), 3032(\mathrm{w}), 2937(\mathrm{~m}), 2862(\mathrm{w}), 2811(\mathrm{w}), 2349(\mathrm{w}), 2297$ (w), 1739 (w), 1644 (s), 1604 (m), 1577 (w), 1553 (w), 1515 (s), 1453 (s), 1382 (m), 1318 (w), 
$1280(\mathrm{~m}), 1249(\mathrm{w}), 1183(\mathrm{w}), 1149(\mathrm{w}), 1098(\mathrm{~s}), 1033(\mathrm{w}), 974(\mathrm{~s}), 926(\mathrm{w}), 853(\mathrm{~m}), 817(\mathrm{w})$, $794(w), 772(w), 743(w), 703(s), 646(s), 599(w), 567(m), 510(w), 468(w)$.

Decomp. $168^{\circ} \mathrm{C}$.

Anal. Calc. for $\mathrm{C}_{46} \mathrm{H}_{38} \mathrm{BF}_{10} \mathrm{PNO}$ : C: $64.80 ; \mathrm{H}: 4.49 ; \mathrm{N}: 1.64$. Found: C: $64.63 ; \mathrm{H}: 4.49 ; \mathrm{N}: 1.69$.

Crystals suitable for the X-ray crystal structure analysis were obtained by slow diffusion of pentane into a solution of compound 19 in dichloromethane at $-35^{\circ} \mathrm{C}$ :

X-ray crystal structure analysis of compound 19: formula $\mathrm{C}_{46} \mathrm{H}_{38} \mathrm{BF}_{10} \mathrm{NOP} \cdot 2 \times \mathrm{CH}_{2} \mathrm{Cl}_{2}, M$ $=1022.41$, colourless crystal, $0.18 \times 0.05 \times 0.05 \mathrm{~mm}, a=12.6441(2), b=14.5097(2), c=$ 15.1347(3) $\AA, \alpha=112.290(1), \beta=96.698(1), y=108.846(2)^{\circ}, V=2339.0(1) \AA^{3}, \rho_{\text {calc }}=1.452$ $\mathrm{gcm}^{-3}, \mu=0.365 \mathrm{~mm}^{-1}$, empirical absorption correction (0.937 $\left.\leq \mathrm{T} \leq 0.982\right), Z=2$, triclinic, space group $P^{i}$ (No. 2), $\lambda=0.71073 \AA, T=223(2) \mathrm{K}, \omega$ and $\varphi$ scans, 20997 reflections collected $( \pm h, \pm k, \pm I), 8004$ independent $\left(R_{\text {int }}=0.045\right)$ and 6444 observed reflections $[>2 \sigma(I)]$, 657 refined parameters, $R=0.070, w R^{2}=0.161$, max. (min.) residual electron density 0.53 ($0.60)$ e. $\AA^{-3}$, hydrogen atoms were calculated and refined as riding atoms.

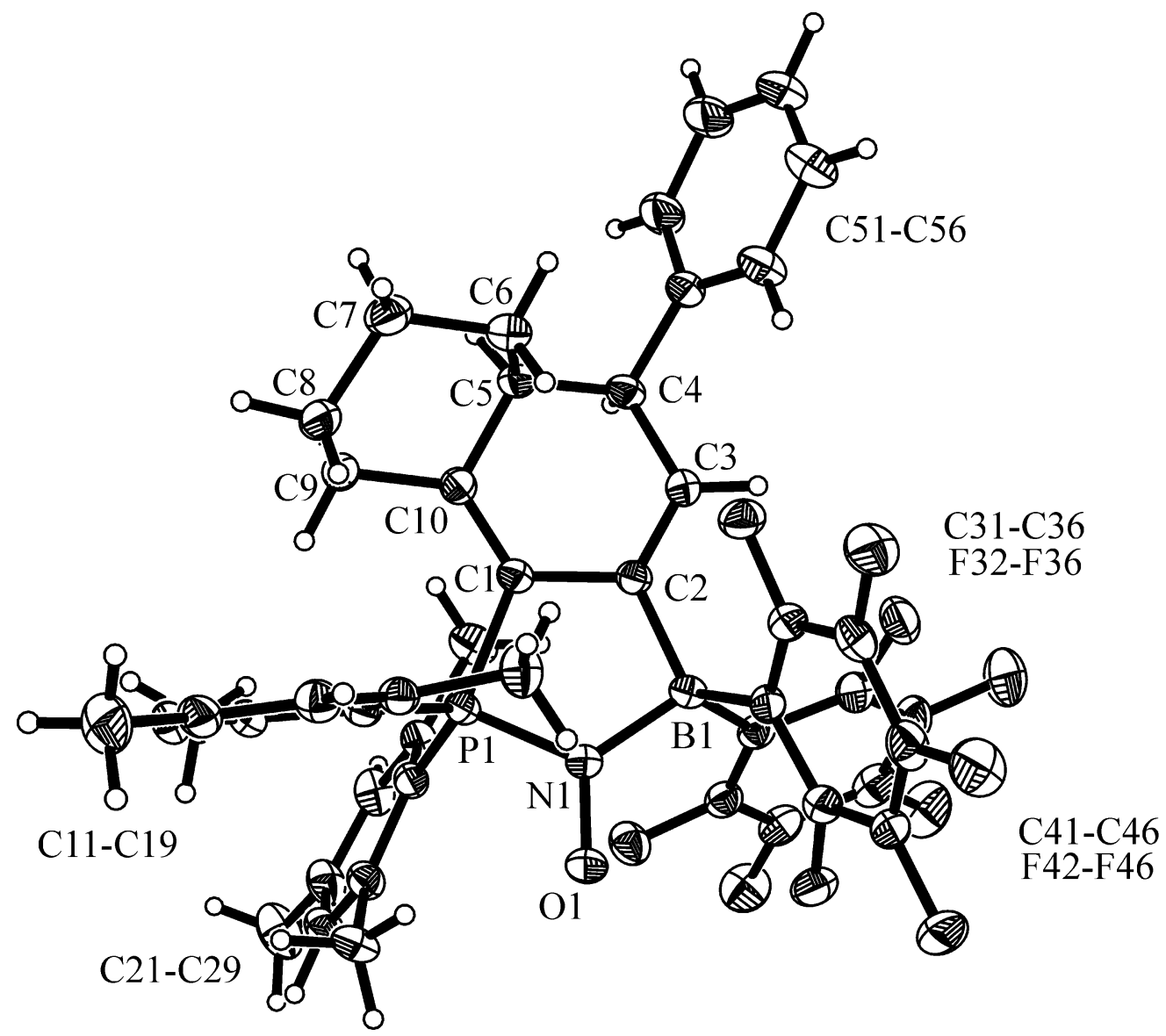

Figure S64: X-ray crystal structure of compound 19 (thermal ellipsoids are shown at the $30 \%$ probability level) 


\section{Synthesis of compound 20}
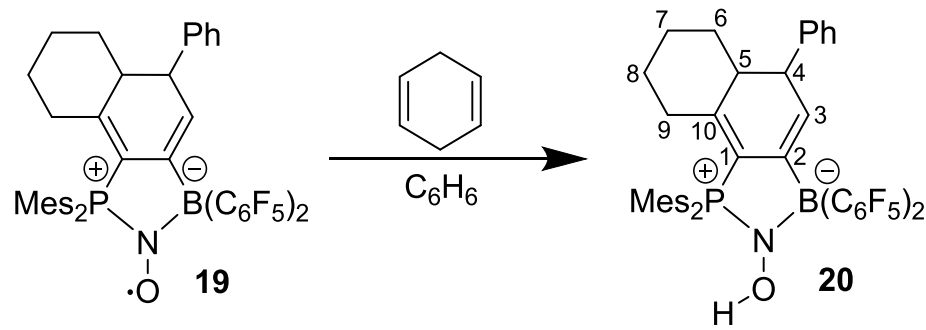

Scheme S12

1,8-Cyclohexadiene (131.6 mg, $1.64 \mathrm{mmol}, 10.0 \mathrm{eq}$ ) was added to a turquoise solution of compound 19 (140.0 mg, $164.2 \mu \mathrm{mol}, 1.0 \mathrm{eq})$ in benzene ( $2 \mathrm{~mL})$ at room temperature. The solution became colorless upon stirring for 1 hour. Then all volatiles were removed in vacuo and the off-white residue crystallized by the "layering method" with $\mathrm{CH}_{2} \mathrm{Cl}_{2}(1 \mathrm{~mL})$ and pentane $(7 \mathrm{~mL})$. The crystalline solid was ground and washed with pentane $(3 \times 1 \mathrm{~mL})$. After drying in vacuo, compound 20 was obtained as a white solid (88.4 mg, $103.6 \mu \mathrm{mol}, 63 \%)$.

IR (KBr): $\tilde{v}\left[\mathrm{~cm}^{-1}\right]=3515(\mathrm{~m}), 3061(\mathrm{w}), 3033(\mathrm{w}), 2936(\mathrm{~m}), 2859(\mathrm{w}), 2816(\mathrm{w}), 2733(\mathrm{w})$, 2340 (w), 1946 (w), 1640 (m), 1603 (m), 1590 (w), 1554 (w), 1512 (s), 1456 (s), 1406 (w), 1385 (w), 1340 (w), 1276 (m), 1246 (w), 1207 (w), 1162 (w), 1139 (w), 1084 (s), 1040 (m), 967 (s), $927(w), 892(w), 852(m), 803(w), 779(w), 754(w), 741(w), 703(m), 689(m), 645(s), 599$ $(w), 572(m), 510(w), 473(w), 452(w)$.

Decomp. $214^{\circ} \mathrm{C}$.

Anal. Calc. for $\mathrm{C}_{46} \mathrm{H}_{39} \mathrm{BF}_{10} \mathrm{PNO}$ : C: $64.73 ; \mathrm{H}: 4.61 ; \mathrm{N}: 1.64$. Found: C: $64.71 ; \mathrm{H}: 4.50 ; \mathrm{N}: 1.58$.

${ }^{1} \mathrm{H}$ NMR (500 MHz, dichloromethane- $\left.d_{2}, 299 \mathrm{~K}\right): \delta 7.33(\mathrm{~m}, 2 \mathrm{H}, m-\mathrm{Ph}), 7.29(\mathrm{~m}, 2 \mathrm{H}, o-\mathrm{Ph})$, $7.23(\mathrm{~m}, 1 \mathrm{H}, p-\mathrm{Ph}), 7.00\left(\mathrm{~d},{ }^{4} \mathrm{~J}_{\mathrm{PH}}=4.3 \mathrm{~Hz}, 2 \mathrm{H}, m-\mathrm{Mes}^{\mathrm{a}}\right), 6.92\left(\mathrm{~d},{ }^{4} J_{\mathrm{PH}}=4.6 \mathrm{~Hz}, 2 \mathrm{H}, m-\mathrm{Mes}^{\mathrm{b}}\right)$, $6.33(\mathrm{br}, 1 \mathrm{H}, 3-\mathrm{CH}), 4.63(\mathrm{t}, J=9.1 \mathrm{~Hz}, 1 \mathrm{H}, \mathrm{OH}), 3.89\left(\mathrm{br} \mathrm{dd},{ }^{3} J_{\mathrm{HH}}=9.5 \mathrm{~Hz}, J=2.0 \mathrm{~Hz}, 1 \mathrm{H}\right.$, 4- $\mathrm{CH}$ ), 2.60/2.13 (each m, each $\left.1 \mathrm{H}, 9-\mathrm{CH}_{2}\right), 2.37$ (s, 6H, o- $\left.\mathrm{CH}_{3}{ }^{\mathrm{Mes}, \mathrm{a}}\right), 2.36\left(\mathrm{~s}, 3 \mathrm{H}, p-\mathrm{CH}_{3}{ }^{\mathrm{Mes}, \mathrm{a}}\right.$ ), $2.33(\mathrm{~m}, 1 \mathrm{H}, 5-\mathrm{CH}), 2.29\left(\mathrm{~s}, 3 \mathrm{H}, p-\mathrm{CH}_{3}{ }^{\mathrm{Mes}, \mathrm{b}}\right), 2.20\left(\mathrm{~s}, 6 \mathrm{H}, o-\mathrm{CH}_{3}{ }^{\mathrm{Mes}, \mathrm{b}}\right), 1.56 / 1.13$ (each m, each $1 \mathrm{H}, 6-\mathrm{CH}_{2}$ ), 1.53/1.14 (each m, each $1 \mathrm{H}, 7-\mathrm{CH}_{2}$ ), 1.27/0.46 (each m, each $1 \mathrm{H}, 8-\mathrm{CH}_{2}$ ). ${ }^{13} \mathrm{C}\left\{{ }^{1} \mathrm{H}\right\}$ NMR $\left(126 \mathrm{MHz}\right.$, dichloromethane- $\left.d_{2}, 299 \mathrm{~K}\right): \delta 159.5\left(\mathrm{~d},{ }^{2} \mathrm{~J}_{\mathrm{PC}}=12.8 \mathrm{~Hz}, 10-\mathrm{C}\right), 144.0$ $\left(\mathrm{d},{ }^{4} J_{\mathrm{PC}}=3.0 \mathrm{~Hz}, p-\mathrm{Mes}^{\mathrm{b}}\right), 143.8\left(\mathrm{~d},{ }^{2} \mathrm{~J}_{\mathrm{PC}}=11.4 \mathrm{~Hz}, o-\mathrm{Mes}^{\mathrm{b}}\right), 143.0\left(\mathrm{~d},{ }^{4} \mathrm{~J}_{\mathrm{PC}}=2.9 \mathrm{~Hz}, p-\mathrm{Mes}^{\mathrm{a}}\right)$, $142.9(i-\mathrm{Ph}), 142.0(\mathrm{br}, 2-\mathrm{C}), 141.6\left(\mathrm{~d},{ }^{2} \mathrm{~J}_{\mathrm{PC}}=10.4 \mathrm{~Hz}, o-\mathrm{Mes}^{\mathrm{a}}\right), 132.7\left(\mathrm{~d},{ }^{3} J_{\mathrm{PC}}=11.7 \mathrm{~Hz}, \mathrm{~m}-\right.$ Mes $\left.^{b}\right), 132.3\left(\mathrm{~d},{ }^{3} \mathrm{JPC}_{\mathrm{PC}}=11.7 \mathrm{~Hz}, m-\mathrm{Mes}^{\mathrm{a}}\right), 129.3(\mathrm{o}-\mathrm{Ph}), 129.2$ (br m, 3-CH), $128.6(m-\mathrm{Ph})$, $126.6(p-\mathrm{Ph}), 124.9\left(\mathrm{~d},{ }^{1} J_{\mathrm{PC}}=86.3 \mathrm{~Hz}, i-\mathrm{Mes}^{\mathrm{a}}\right), 122.5\left(\mathrm{~d},{ }^{1} J_{\mathrm{PC}}=90.0 \mathrm{~Hz}, i-\mathrm{Mes}^{\mathrm{b}}\right), 120.4\left(\mathrm{~d},{ }^{1} J_{\mathrm{PC}}\right.$ $=99.3 \mathrm{~Hz}, 1-\mathrm{C}), 50.5\left(\mathrm{~d},{ }^{3} J_{\mathrm{PC}}=13.3 \mathrm{~Hz}, 5-\mathrm{CH}\right), 44.1(4-\mathrm{CH}), 35.6\left(\mathrm{~d},{ }^{3} J_{\mathrm{PC}}=7.3 \mathrm{~Hz}, 9-\mathrm{CH}_{2}\right)$, $30.2\left(\mathrm{br} \mathrm{d},{ }^{4} \mathrm{~J}_{\mathrm{PC}}=1.9 \mathrm{~Hz}, 8-\mathrm{CH}_{2}\right), 30.0\left(\mathrm{br}, 6-\mathrm{CH}_{2}\right), 26.9\left(7-\mathrm{CH}_{2}\right), 24.2\left(\mathrm{~d},{ }^{3} \mathrm{JPC}_{\mathrm{PC}}=4.2 \mathrm{~Hz}, 0-\right.$ 
$\left.\mathrm{CH}_{3}{ }^{\mathrm{Mes}, \mathrm{a}}\right), 23.8\left(\mathrm{~d},{ }^{3} \mathrm{JPC}=5.4 \mathrm{~Hz}, o-\mathrm{CH}_{3}{ }^{\mathrm{Mes}, \mathrm{b}}\right), 21.2\left(\mathrm{~d}, J=1.9 \mathrm{~Hz}, p-\mathrm{CH}_{3}{ }^{\mathrm{Mes}, \mathrm{b}}\right), 21.2(\mathrm{~d}, J=1.3 \mathrm{~Hz}$, $\left.p-\mathrm{CH}_{3}{ }^{\mathrm{Mes}, \mathrm{a}}\right) \cdot\left[\mathrm{C}_{6} \mathrm{~F}_{5}\right.$ not listed]

${ }^{31} \mathbf{P}\left\{{ }^{1} \mathrm{H}\right\}$ NMR $\left(202 \mathrm{MHz}\right.$, dichloromethane- $\left.d_{2}, 299 \mathrm{~K}\right): \delta 28.7\left(v_{1 / 2} \sim 25 \mathrm{~Hz}\right)$.

${ }^{11} \mathbf{B}\left\{{ }^{1} \mathrm{H}\right\}$ NMR $\left(160 \mathrm{MHz}\right.$, dichloromethane- $\left.d_{2}, 299 \mathrm{~K}\right): \delta-6.6\left(v_{1 / 2} \sim 130 \mathrm{~Hz}\right)$

${ }^{19} \mathrm{~F}$ NMR $\left(470 \mathrm{MHz}\right.$, dichloromethane- $\left.d_{2}, 299 \mathrm{~K}\right): \delta-128.7(\mathrm{~m}, 2 \mathrm{~F}, \mathrm{o}),-161.5\left(\mathrm{t},{ }^{3} \mathrm{JFF}=20.3 \mathrm{~Hz}\right.$, $1 \mathrm{~F}, p),-165.9(\mathrm{~m}, 2 \mathrm{~F}, m)\left(\mathrm{C}_{6} \mathrm{~F}_{5}\right)\left[\Delta \delta^{19} \mathrm{~F}_{\mathrm{mp}}=4.4\right],-136.8(\mathrm{~m}, 2 \mathrm{~F}, o),-162.6\left(\mathrm{t},{ }^{3} J_{\mathrm{FF}}=20.3 \mathrm{~Hz}, 1 \mathrm{~F}\right.$, $p),-165.3(\mathrm{~m}, 2 \mathrm{~F}, m)\left(\mathrm{C}_{6} \mathrm{~F}_{5}\right)\left[\Delta \delta^{19} \mathrm{~F}_{\mathrm{mp}}=2.7\right]$.

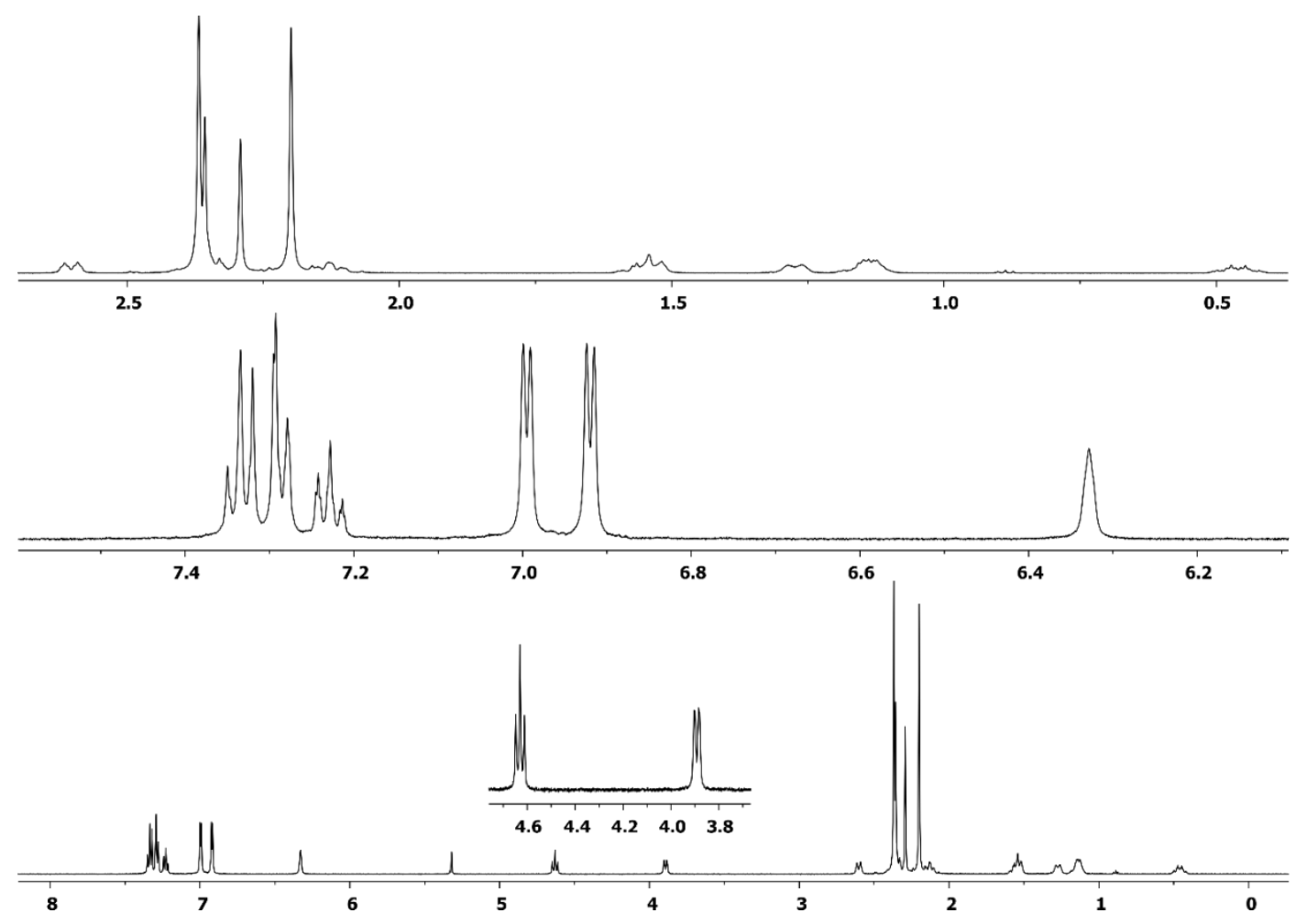

Figure S65: ${ }^{1} \mathrm{H}$ NMR (500 MHz, dichloromethane- $\left.d_{2}, 299 \mathrm{~K}\right)$ spectrum of compound 20 

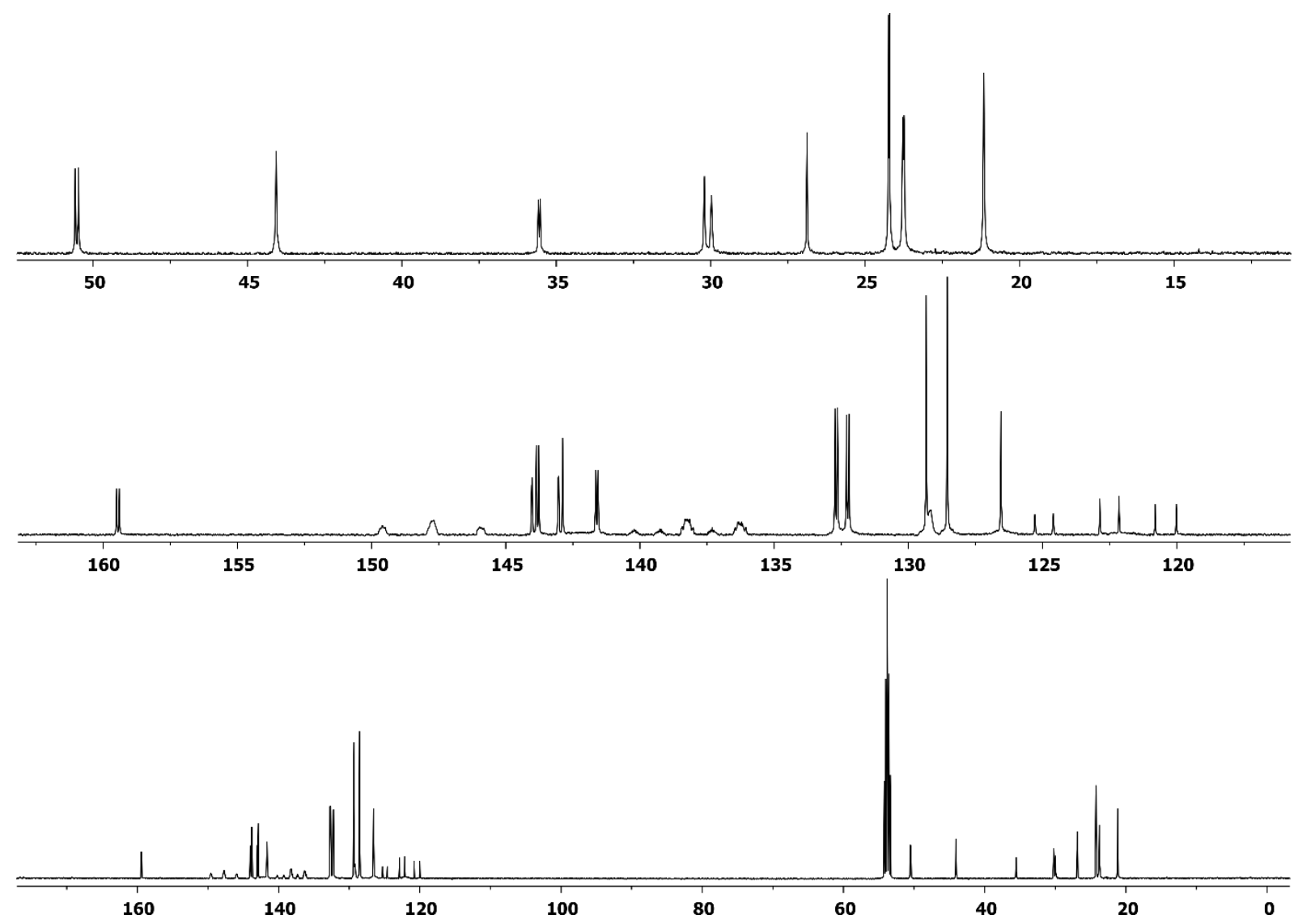

Figure S66: ${ }^{13} \mathbf{C}\left\{{ }^{1} \mathrm{H}\right\}$ NMR $\left(126 \mathrm{MHz}\right.$, dichloromethane- $\left.d_{2}, 299 \mathrm{~K}\right)$ spectrum of compound 20

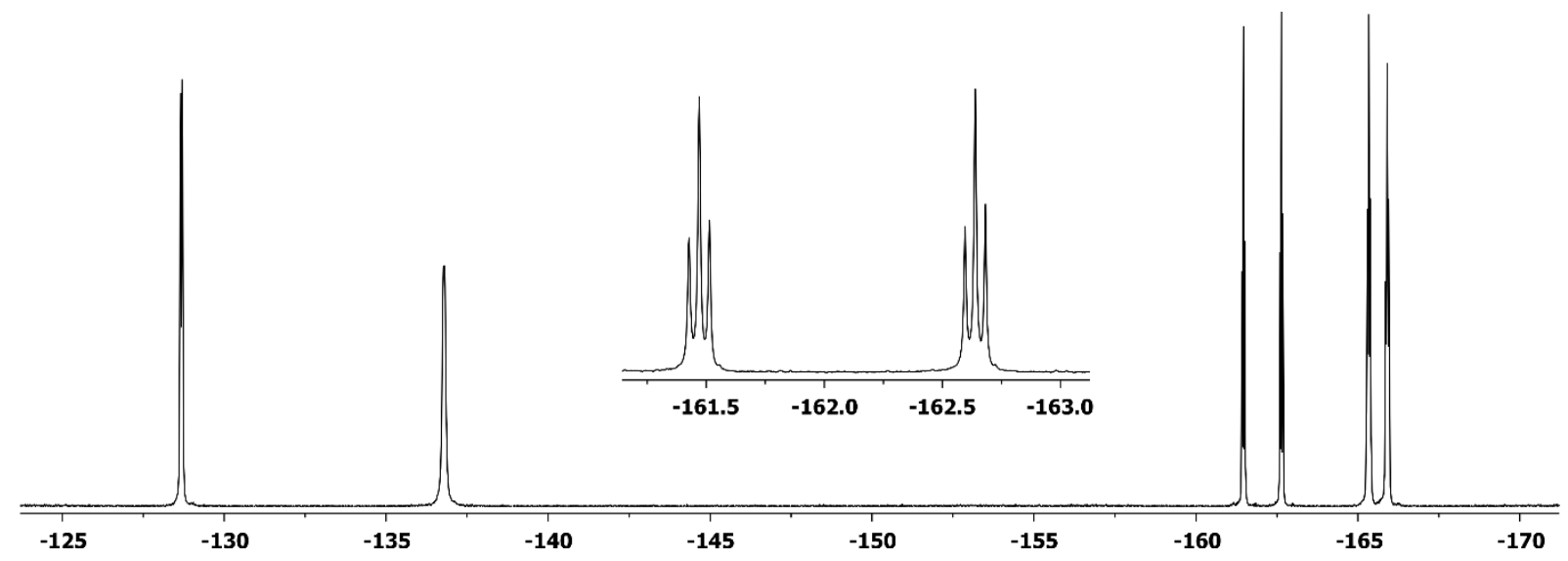

Figure S67: ${ }^{19} \mathrm{~F}$ NMR (470 MHz, dichloromethane-d2, $\left.299 \mathrm{~K}\right)$ spectrum of compound 20

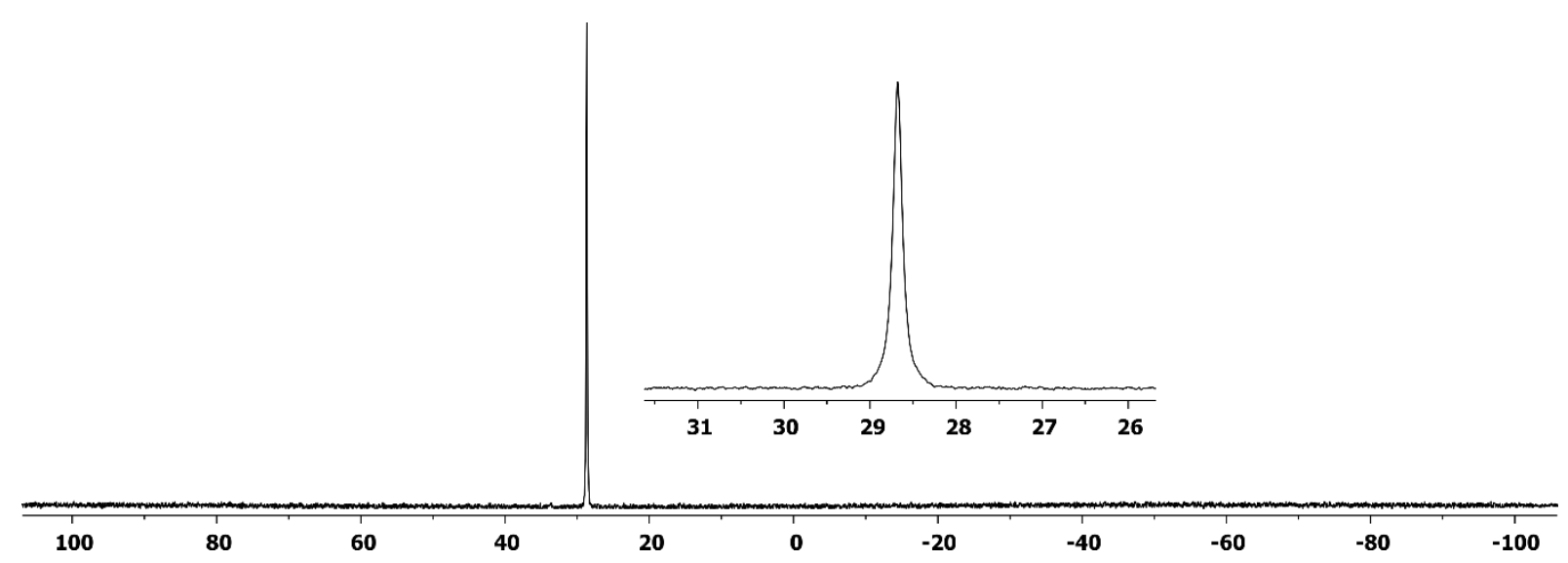

Figure $\mathbf{S} 68{ }^{31} \mathrm{P}\left\{{ }^{1} \mathrm{H}\right\}$ NMR (202 MHz, dichloromethane- $\left.d_{2}, 299 \mathrm{~K}\right)$ spectrum of compound 20 


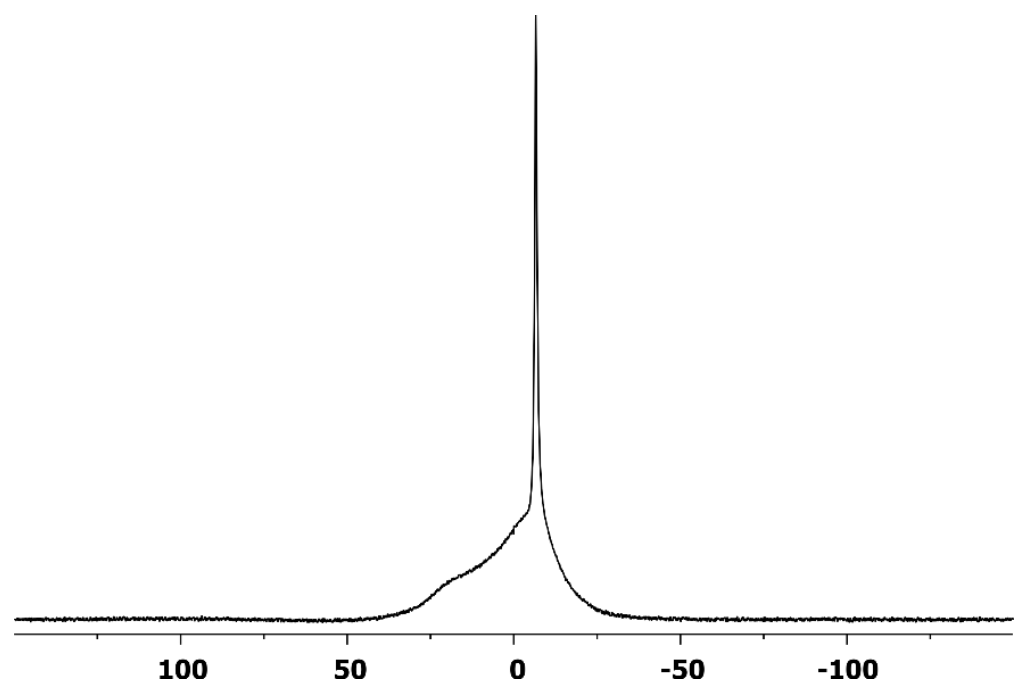

Figure S69: ${ }^{11} \mathbf{B}\left\{{ }^{1} \mathbf{H}\right\}$ NMR (160 MHz, dichloromethane-d2, $\left.299 \mathrm{~K}\right)$ spectrum of compound 20

Crystals suitable for the X-ray crystal structure analysis were obtained by slow evaporation from a dichloromethane solution of compound $\mathbf{2 0}$ :

X-ray crystal structure analysis of compound 20: A colorless prism-like specimen of $\mathrm{C}_{46} \mathrm{H}_{39} \mathrm{BF}_{10} \mathrm{NOP}$, approximate dimensions $0.080 \mathrm{~mm} \times 0.180 \mathrm{~mm} \times 0.200 \mathrm{~mm}$, was used for the $\mathrm{X}$-ray crystallographic analysis. The X-ray intensity data were measured. A total of 1605 frames were collected. The total exposure time was 22.24 hours. The frames were integrated with the Bruker SAINT software package using a wide-frame algorithm. The integration of the data using a monoclinic unit cell yielded a total of 57199 reflections to a maximum $\theta$ angle of $66.59^{\circ}$ (0.84 A resolution), of which 7063 were independent (average redundancy 8.098, completeness $\left.=99.2 \%, R_{\text {int }}=7.46 \%, R_{\text {sig }}=3.97 \%\right)$ and $5503(77.91 \%)$ were greater than $2 \sigma\left(F^{2}\right)$. The final cell constants of $\underline{a}=12.5817(5) \AA, \underline{b}=15.0138(6) \AA, \underline{c}=22.1390(9) \AA, \beta=$ $105.279(2)^{\circ}$, volume $=4034.2(3) \AA^{3}$, are based upon the refinement of the $X Y Z$-centroids of 9971 reflections above $20 \sigma(\mathrm{I})$ with $7.197^{\circ}<2 \theta<135.7^{\circ}$. Data were corrected for absorption effects using the multi-scan method (SADABS). The ratio of minimum to maximum apparent transmission was 0.841 . The calculated minimum and maximum transmission coefficients (based on crystal size) are 0.7750 and 0.9000 . The structure was solved and refined using the Bruker SHELXTL Software Package, using the space group P $121 / n 1$, with $Z=4$ for the formula unit, $\mathrm{C}_{46} \mathrm{H}_{39} \mathrm{BF} \mathrm{F}_{10} \mathrm{NOP}$. The final anisotropic full-matrix least-squares refinement on $\mathrm{F}^{2}$ with 551 variables converged at $R 1=4.75 \%$, for the observed data and $w R 2=12.71 \%$ for all data. The goodness-of-fit was 1.072. The largest peak in the final difference electron density synthesis was $0.744 \mathrm{e}^{-} / \AA^{3}$ and the largest hole was $-0.363 \mathrm{e}^{-} / \AA^{3}$ with an RMS deviation of 0.060 $\mathrm{e}^{-} / \AA^{3}$. On the basis of the final model, the calculated density was $1.405 \mathrm{~g} / \mathrm{cm}^{3}$ and $F(000)$, $1760 \mathrm{e}^{-}$. 


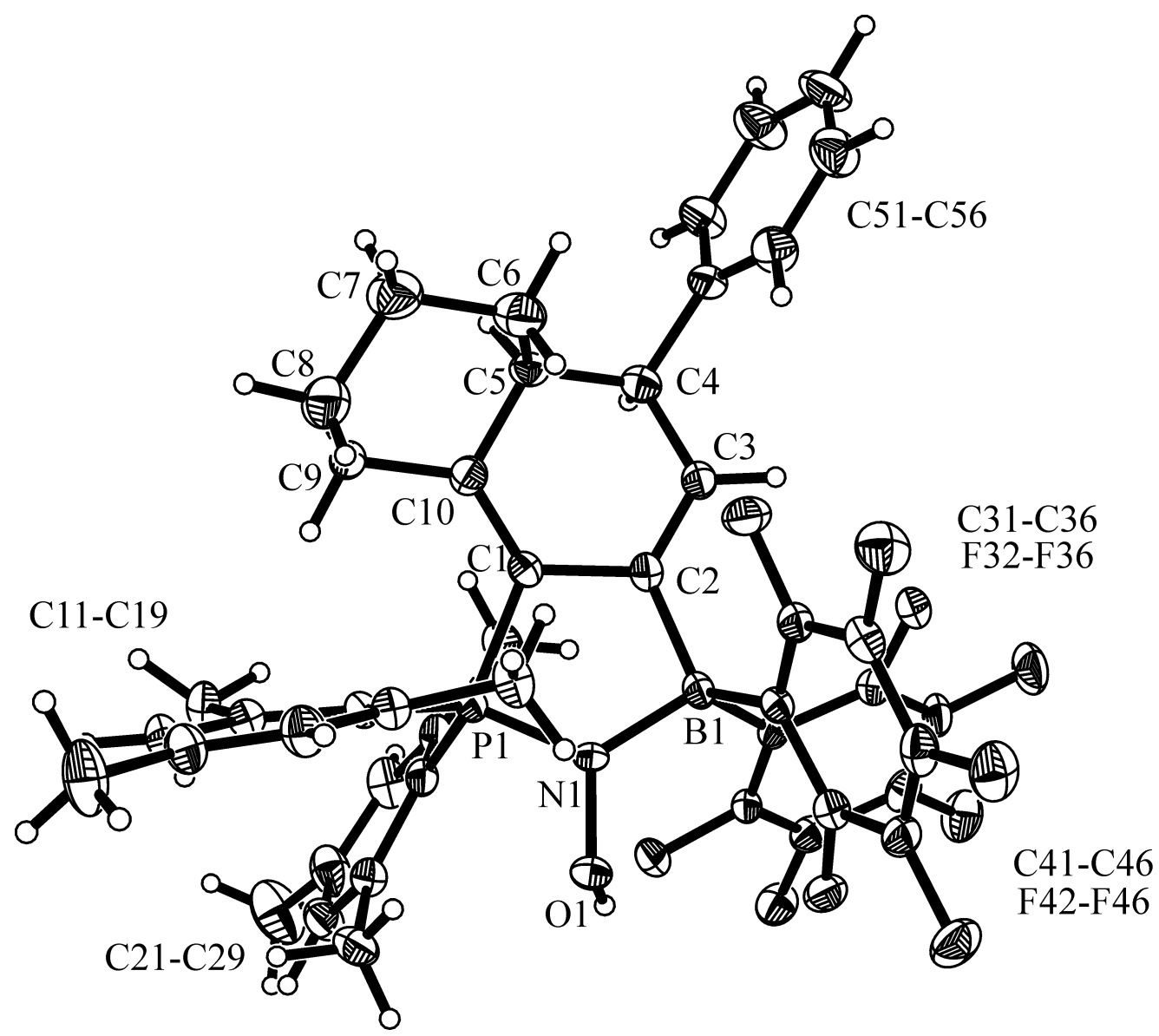

Figure S70: X-ray crystal structure of compound 20 (thermal ellipsoids are shown at the 50\% probability level)

\section{Synthesis of compound 21}

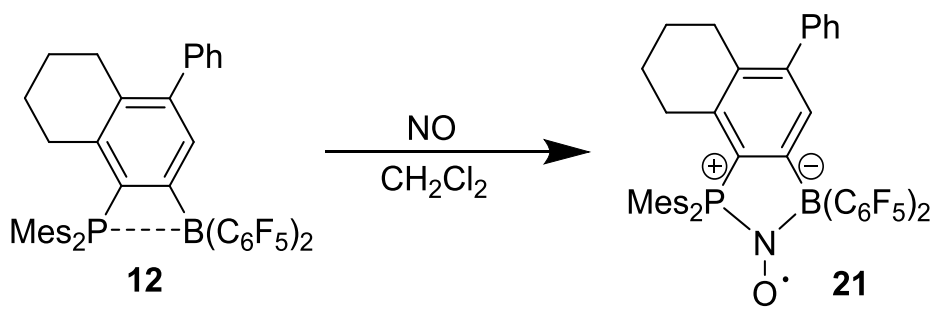

Scheme S13

A solution of compound $12(100.0 \mathrm{mg}, 121.9 \mu \mathrm{mol}, 1.0 \mathrm{eq})$ in $\mathrm{CH}_{2} \mathrm{Cl}_{2}(1 \mathrm{~mL})$ was degassed at $-78{ }^{\circ} \mathrm{C}$ and exposed to a nitric oxide atmosphere (1.5 bar). The solution turned dark green upon stirring for $15 \mathrm{~min}$. Then all volatiles were removed in vacuo and pentane $(3 \mathrm{~mL})$ was added to the obtained dark green residue. The suspension was filtrated via cannula (Whatman glass fiber filter) and the remaining solid was washed with pentane $(3 \times 2 \mathrm{~mL})$. After drying in vacuo compound 21 was obtained as a pale green solid (87.4 mg, $91.9 \mu \mathrm{mol}$, 84\%).

IR $(\mathrm{KBr}): \tilde{v}\left[\mathrm{~cm}^{-1}\right]=3518(\mathrm{w}), 3455(\mathrm{w}), 3031(\mathrm{w}), 2940(\mathrm{~m}), 2864(\mathrm{w}), 2741(\mathrm{w}), 2624(\mathrm{w}), 2386$ (w), $2348(w), 2302(w), 1732(w), 1642(m), 1604(m), 1554(w), 1515(s), 1459(s), 1382(m)$, $1322(w), 1283(m), 1253(m), 1205(w), 1178(w), 1157(w), 1103(s), 1029(w), 974(s), 926$ 
(w), $881(w), 850(m), 836(m), 809(w), 772(m), 748(w), 702(m), 659(m), 643(m), 609(w)$, $571(w), 515(w), 478(w), 455(w), 429(w)$.

Decomp. $193^{\circ} \mathrm{C}$.

Anal. Calc. for $\mathrm{C}_{46} \mathrm{H}_{36} \mathrm{BF}_{10} \mathrm{PNO}$ : C: 64.96; $\mathrm{H}: 4.27 ; \mathrm{N}: 1.65$. Found: C: 65.34; $\mathrm{H}: 4.28 ; \mathrm{N}: 1.31$.

Crystals suitable for the X-ray crystal structure analysis were obtained by slow diffusion of pentane into a solution of compound 21 in dichloromethane at $-35^{\circ} \mathrm{C}$ :

X-ray crystal structure analysis of compound 21: formula $\mathrm{C}_{46} \mathrm{H}_{36} \mathrm{BF}_{10} \mathrm{NOP}, M=850.54$, pale green crystal, $0.15 \times 0.12 \times 0.10 \mathrm{~mm}, a=13.9970(3), b=14.5832(3), c=19.9467(4) \AA$, $\beta=105.140(1)^{\circ}, V=3930.2(1) \AA^{3}, \rho_{\text {calc }}=1.437 \mathrm{gcm}^{-3}, \mu=0.156 \mathrm{~mm}^{-1}$, empirical absorption correction (0.976 $\leq \mathrm{T} \leq 0.984), Z=4$, monoclinic, space group $P 2_{1} / \mathrm{n}$ (No. 14), $\lambda=0.71073 \AA$, $T=223(2) \mathrm{K}, \omega$ and $\varphi$ scans, 23571 reflections collected $( \pm h, \pm k, \pm 1), 6794$ independent $\left(R_{\text {int }}=\right.$ $0.053)$ and 5010 observed reflections $[/>2 \sigma(I)], 639$ refined parameters, $R=0.066, w R^{2}=$ 0.139 , max. (min.) residual electron density $0.31(-0.29)$ e. $\AA^{-3}$, hydrogen atoms were calculated and refined as riding atoms.

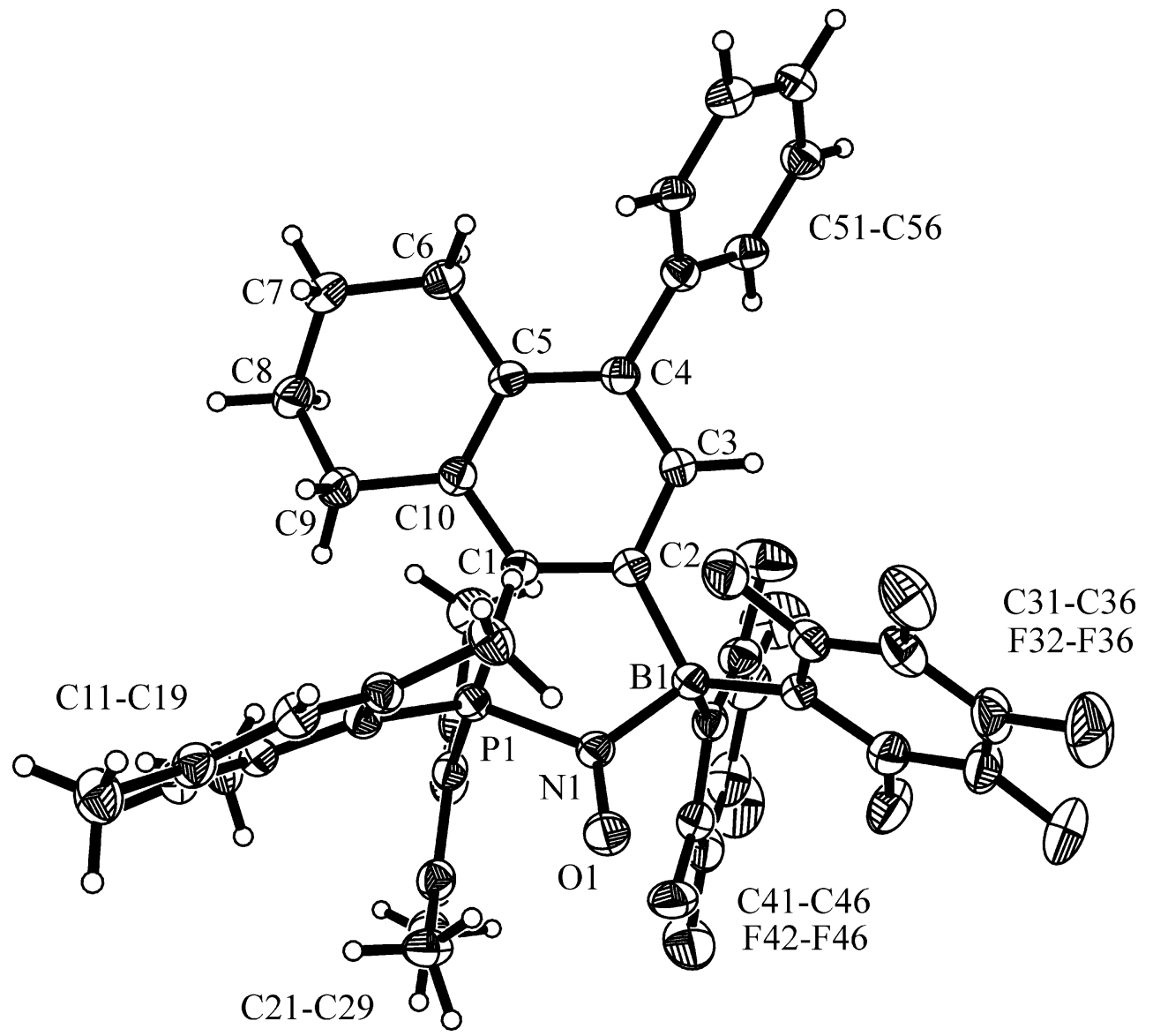

Figure S71: X-ray crystal structure of compound 21 (thermal ellipsoids are shown at the 30\% probability level) 


\section{Synthesis of compound 22}
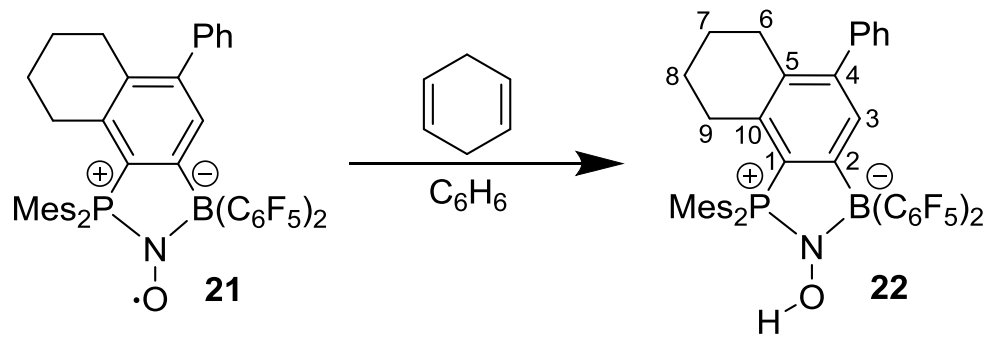

Scheme S14

1,8-Cyclohexadiene $(75.4 \mathrm{mg}, 940.6 \mu \mathrm{mol}, 10.0 \mathrm{eq})$ was added to a green solution of compound $21(80.0 \mathrm{mg}, 94.1 \mu \mathrm{mol}, 1.0 \mathrm{eq})$ in benzene $(2 \mathrm{~mL})$ at room temperature. The solution became colorless upon stirring for 1 hour. Then all volatiles were removed in vacuo and the remaining off-white residue was washed with pentane $(3 \times 1 \mathrm{~mL})$. After drying in vacuo, compound 22 was obtained as a white solid (70.8 mg, $83.1 \mu \mathrm{mol}, 88 \%)$.

IR $(\mathrm{KBr}): \tilde{v}\left[\mathrm{~cm}^{-1}\right]=3494(\mathrm{w}), 2934(\mathrm{w}), 2864(\mathrm{w}), 1642(\mathrm{~m}), 1604(\mathrm{~m}), 1556(\mathrm{w}), 1515(\mathrm{~s}), 1456$ (s), $1381(\mathrm{w}), 1342(\mathrm{w}), 1227(\mathrm{~m}), 1252(\mathrm{~m}), 1212(\mathrm{w}), 1159(\mathrm{w}), 1090(\mathrm{~s}), 1041$ (w), 971 (s), $933(w), 853(m), 822(w), 775(m), 741(w), 703(m), 659(m), 642(m), 576(w), 507(w), 482$ $(w), 440(w)$.

Decomp. $220^{\circ} \mathrm{C}$.

Anal. Calc. for $\mathrm{C}_{46} \mathrm{H}_{37} \mathrm{BF}_{10} \mathrm{PNO}$ : C: $64.88 ; \mathrm{H}: 4.38 ; \mathrm{N}: 1.64$. Found: C: $65.00 ; \mathrm{H}: 4.44 ; \mathrm{N}: 1.50$.

${ }^{1} \mathrm{H}$ NMR $\left(500 \mathrm{MHz}\right.$, dichloromethane- $\left.d_{2}, 299 \mathrm{~K}\right): \delta 7.82(\mathrm{br}, 1 \mathrm{H}, 3-\mathrm{CH}), 7.42(\mathrm{~m}, 2 \mathrm{H}, m-\mathrm{Ph})$, $7.35(\mathrm{~m}, 1 \mathrm{H}, p-\mathrm{Ph}), 7.33(\mathrm{~m}, 2 \mathrm{H}, o-\mathrm{Ph}), 6.97$ (d, $\left.{ }^{4} \mathrm{~J}_{\mathrm{PH}}=4.4 \mathrm{~Hz}, 4 \mathrm{H}, m-\mathrm{Mes}\right), 4.76$ (quint, $J=$ $5.0 \mathrm{~Hz}, 1 \mathrm{H}, \mathrm{OH}), 2.68\left(\mathrm{~m}, 2 \mathrm{H}, 9-\mathrm{CH}_{2}\right), 2.56\left(\mathrm{~m}, 2 \mathrm{H}, 6-\mathrm{CH}_{2}\right), 2.33\left(\mathrm{~s}, 6 \mathrm{H}, p-\mathrm{CH}_{3}{ }^{\mathrm{Mes}}\right), 2.13(\mathrm{~s}$, $\left.12 \mathrm{H}, o-\mathrm{CH}_{3}{ }^{\mathrm{Mes}}\right), 1.67\left(\mathrm{~m}, 2 \mathrm{H}, 8-\mathrm{CH}_{2}\right), 1.57\left(\mathrm{~m}, 2 \mathrm{H}, 7-\mathrm{CH}_{2}\right)$.

${ }^{13} \mathrm{C}\left\{{ }^{1} \mathrm{H}\right\}$ NMR $\left(126 \mathrm{MHz}\right.$, dichloromethane- $\left.d_{2}, 299 \mathrm{~K}\right): \delta 156.5(\mathrm{br}, 2-\mathrm{C}), 148.0\left(\mathrm{dm},{ }^{1} \mathrm{JFC}^{\sim}\right.$ $\left.240 \mathrm{~Hz}, \mathrm{C}_{6} \mathrm{~F}_{5}\right), 138.2\left(\mathrm{dm}, 146.5\left(\mathrm{~d},{ }^{4} \mathrm{~J}_{\mathrm{PC}}=3.1 \mathrm{~Hz}, 4-\mathrm{CH}\right), 143.6\left(\mathrm{~d},{ }^{4} \mathrm{~J}_{\mathrm{PC}}=2.9 \mathrm{~Hz}, p-\mathrm{Mes}\right), 143.2\right.$ (br d, $\left.{ }^{2} J_{\mathrm{PC}}=10.8 \mathrm{~Hz}, \mathrm{o}-\mathrm{Mes}\right), 142.3(\mathrm{~d}, J=0.9 \mathrm{~Hz}, i-\mathrm{Ph}), 138.2$ (d, $\left.{ }^{2} J_{\mathrm{PC}}=16.6 \mathrm{~Hz}, 10-\mathrm{C}\right), 138.2$ $\left(\mathrm{dm},{ }^{1} \mathrm{~J}_{\mathrm{FC}} \sim 240 \mathrm{~Hz}, \mathrm{C}_{6} \mathrm{~F}_{5}\right), 137.2\left(\mathrm{dm},{ }^{1} \mathrm{~J}_{\mathrm{FC}} \sim 250 \mathrm{~Hz}, \mathrm{C}_{6} \mathrm{~F}_{5}\right), 134.7\left(\mathrm{~d},{ }^{3} J_{\mathrm{PC}}=12.2 \mathrm{~Hz}, 5-\mathrm{C}\right), 133.3$ $(\mathrm{m}, 3-\mathrm{CH}), 132.7$ (d, $\left.{ }^{3} \mathrm{JPC}_{\mathrm{PC}}=11.6 \mathrm{~Hz}, \mathrm{~m}-\mathrm{Mes}\right), 129.5(\mathrm{o}-\mathrm{Ph}), 128.4(\mathrm{~m}-\mathrm{Ph}), 128.3$ (d, ${ }^{1} \mathrm{~J}_{\mathrm{PC}}=$ $100.5 \mathrm{~Hz}, 1-\mathrm{C}), 127.5$ (br, $i-\mathrm{C}_{6} \mathrm{~F}_{5}$ ), 127.4 (p-Ph), 122.2 (d, $\left.{ }^{1} J_{\mathrm{PC}}=87.4 \mathrm{~Hz}, i-\mathrm{Mes}\right), 30.3$ (d, ${ }^{3} J_{\mathrm{PC}}$ $\left.=5.6 \mathrm{~Hz}, 9-\mathrm{CH}_{2}\right), 29.0\left(\mathrm{~d},{ }^{4} \mathrm{~J}_{\mathrm{PC}}=1.8 \mathrm{~Hz}, 6-\mathrm{CH}_{2}\right), 23.5\left(\mathrm{br} \mathrm{d},{ }^{3} \mathrm{~J}_{\mathrm{PC}}=3.6 \mathrm{~Hz}, 0-\mathrm{CH}_{3}{ }^{\mathrm{Mes}}\right), 23.0(8-$ $\left.\mathrm{CH}_{2}\right), 22.9\left(7-\mathrm{CH}_{2}\right), 21.2\left(\mathrm{~d}, J=1.5 \mathrm{~Hz}, p-\mathrm{CH}_{3}{ }^{\mathrm{Mes}}\right)$.

${ }^{31} \mathbf{P}\left\{{ }^{1} \mathrm{H}\right\}$ NMR $\left(202 \mathrm{MHz}\right.$, dichloromethane- $\left.d_{2}, 299 \mathrm{~K}\right): \delta 33.7\left(v_{1 / 2} \sim 40 \mathrm{~Hz}\right)$.

${ }^{11} \mathbf{B}\left\{{ }^{1} \mathrm{H}\right\}$ NMR $\left(160 \mathrm{MHz}\right.$, dichloromethane- $\left.d_{2}, 299 \mathrm{~K}\right): \delta-5.4\left(v_{1 / 2} \sim 150 \mathrm{~Hz}\right)$

${ }^{19} \mathrm{~F}$ NMR $\left(470 \mathrm{MHz}\right.$, dichloromethane- $\left.d_{2}, 299 \mathrm{~K}\right): \delta-131.7\left(\mathrm{br}, 2 \mathrm{~F}, \mathrm{o}-\mathrm{C}_{6} \mathrm{~F}_{5}\right),-161.2\left(\mathrm{t},{ }^{3} \mathrm{JFF}_{\mathrm{FF}}=\right.$ $\left.20.3 \mathrm{~Hz}, 1 \mathrm{~F}, p-\mathrm{C}_{6} \mathrm{~F}_{5}\right),-165.4\left(\mathrm{~m}, 2 \mathrm{~F}, m-\mathrm{C}_{6} \mathrm{~F}_{5}\right)\left[\Delta \delta^{19} \mathrm{~F}_{\mathrm{mp}}=4.2\right]$. 

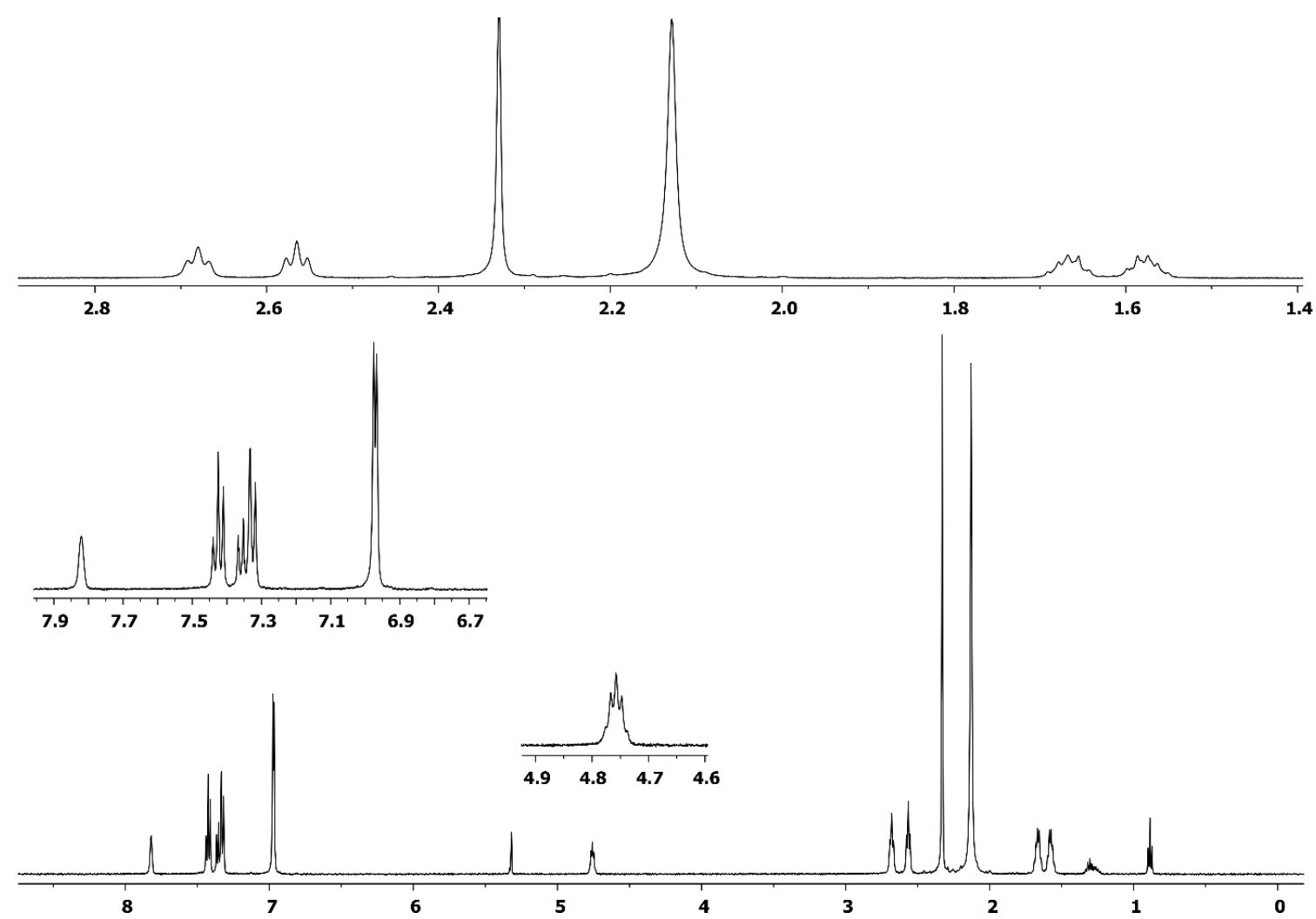

Figure S72: ${ }^{1} \mathbf{H}$ NMR (500 MHz, dichloromethane- $d_{2}, 299 \mathrm{~K}$ ) spectrum of compound 22

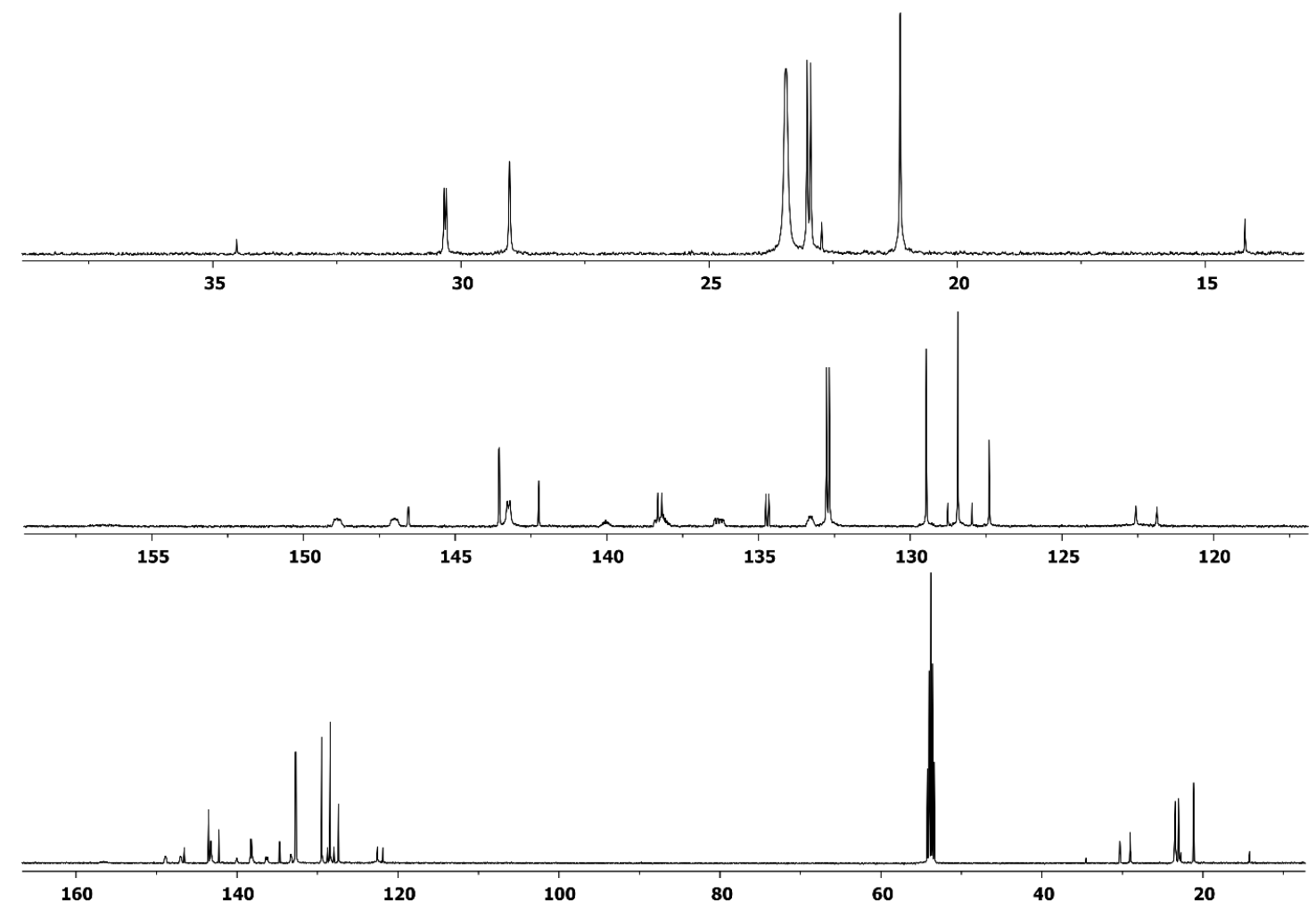

Figure S73: ${ }^{13} \mathbf{C}\left\{{ }^{1} \mathbf{H}\right\}$ NMR (126 MHz, dichloromethane- $\left.d_{2}, 299 \mathrm{~K}\right)$ spectrum of compound 22 


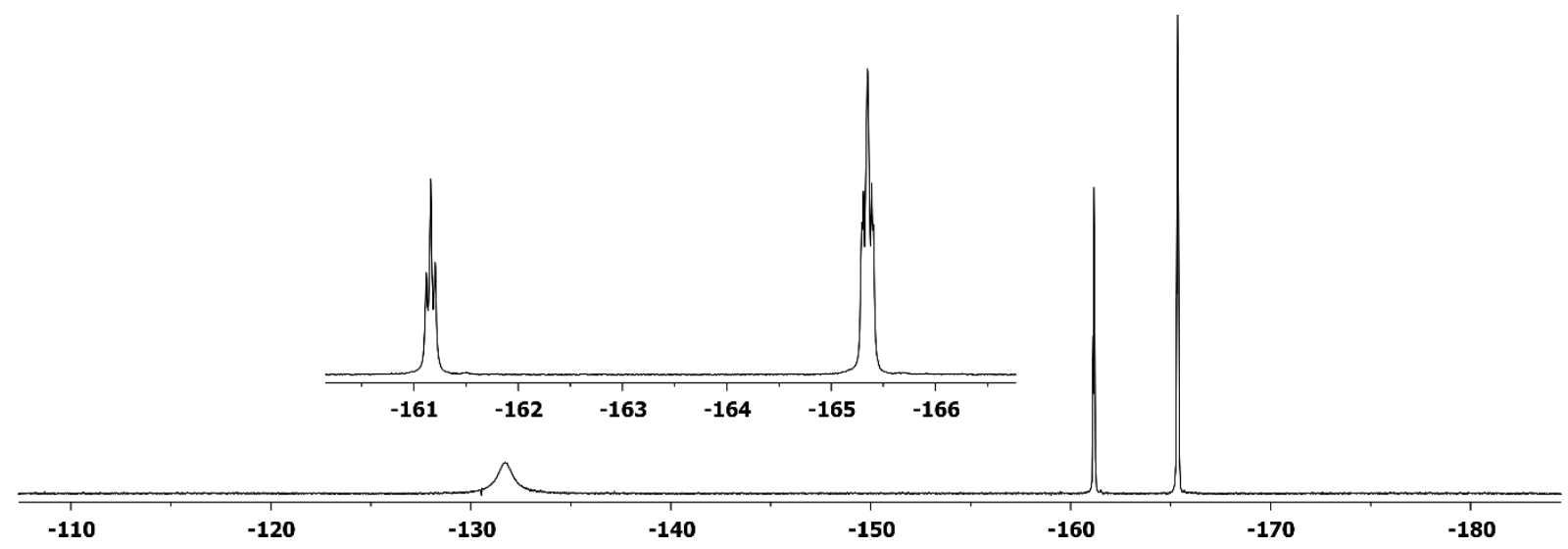

Figure S74: ${ }^{19} \mathrm{~F}$ NMR (470 MHz, dichloromethane-d2, $299 \mathrm{~K}$ ) spectrum of compound 22

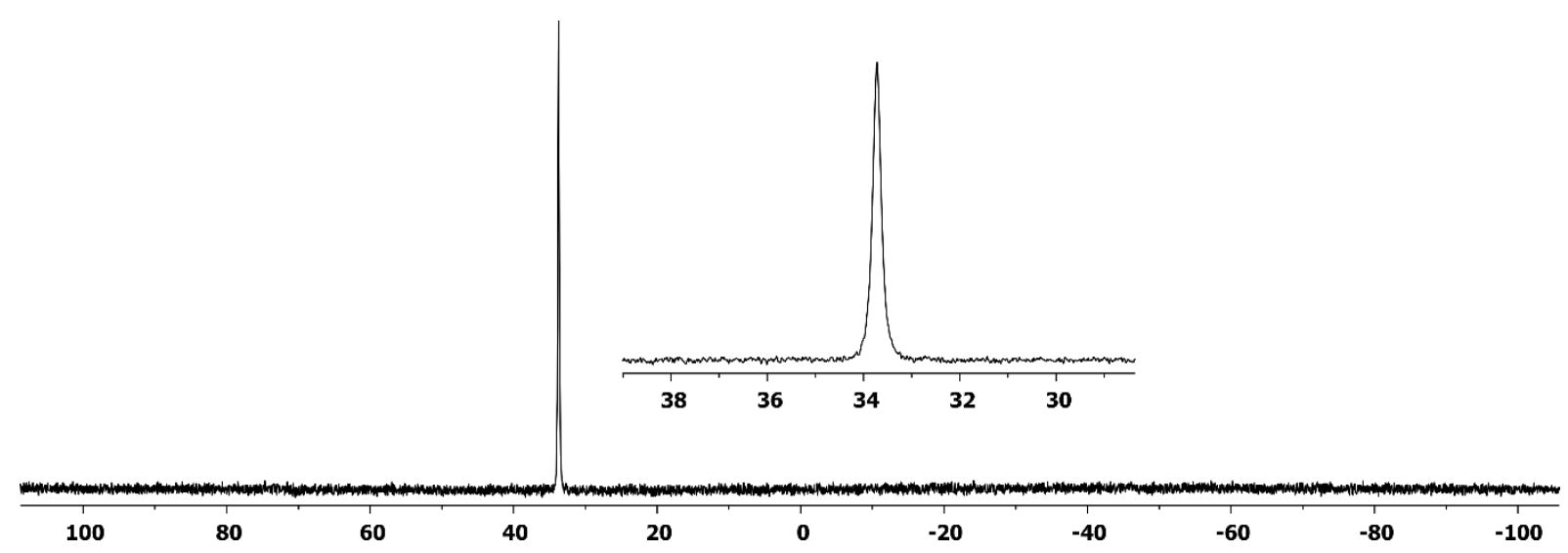

Figure S75: ${ }^{31} \mathrm{P}\left\{{ }^{1} \mathrm{H}\right\}$ NMR (202 MHz, dichloromethane- $\left.d_{2}, 299 \mathrm{~K}\right)$ spectrum of compound 22

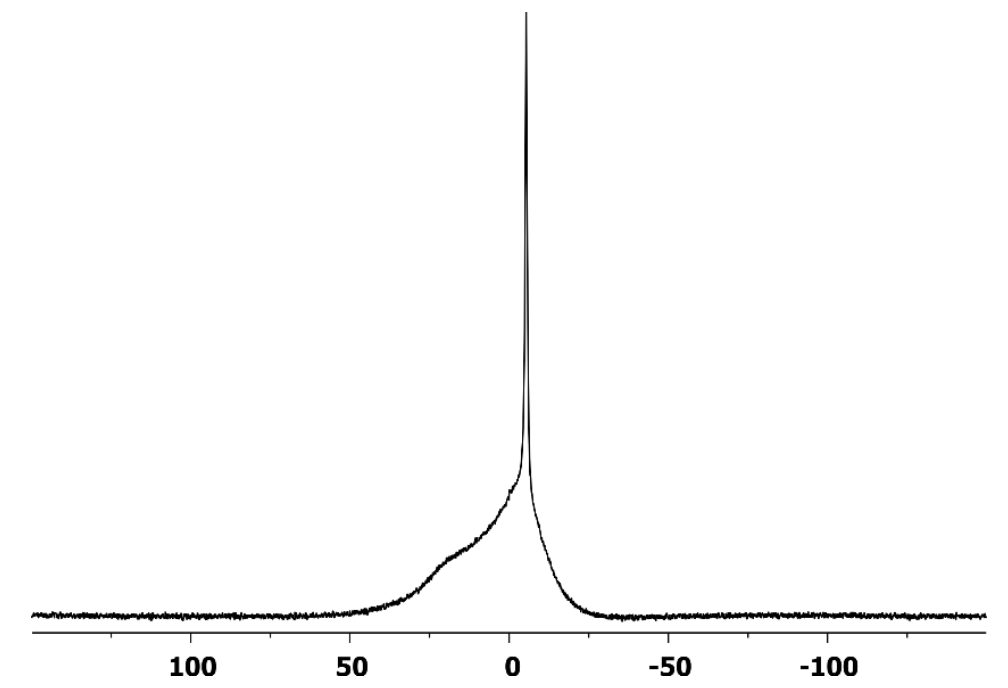

Figure S76: ${ }^{11} \mathbf{B}\left\{{ }^{1} \mathbf{H}\right\}$ NMR $\left(160 \mathrm{MHz}\right.$, dichloromethane- $\left.d_{2}, 299 \mathrm{~K}\right)$ spectrum of compound 22 
Crystals suitable for the X-ray crystal structure analysis were obtained by slow evaporation a solution of compound $\mathbf{2 2}$ in dichloromethane at room temperature:

X-ray crystal structure analysis of compound 22: formula $\mathrm{C}_{46} \mathrm{H}_{37} \mathrm{BF}_{10} \mathrm{NOP}, M=851.55$, colourless crystal, $0.20 \times 0.12 \times 0.06 \mathrm{~mm}, a=17.1332(2), b=14.0711(2), c=17.4447$ (3) $\AA, \beta$ $=106.398(1)^{\circ}, V=4034.5(1) \AA^{3}, \rho_{\text {calc }}=1.402 \mathrm{gcm}^{-3}, \mu=0.152 \mathrm{~mm}^{-1}$, empirical absorption correction (0.970 $\leq \mathrm{T} \leq 0.990), Z=4$, monoclinic, space group $P 2 / \mathrm{n}$ (No. 14$), \lambda=0.71073 \AA$, $T=223(2) \mathrm{K}, \omega$ and $\varphi$ scans, 22804 reflections collected $( \pm h, \pm k, \pm)$, 6988 independent $\left(R_{\text {int }}=\right.$ $0.058)$ and 4841 observed reflections $[>2 \sigma(I)], 548$ refined parameters, $R=0.070, w R^{2}=$ 0.143 , max. (min.) residual electron density $0.26(-0.32)$ e. $\AA^{-3}$, hydrogen atoms were calculated and refined as riding atoms.

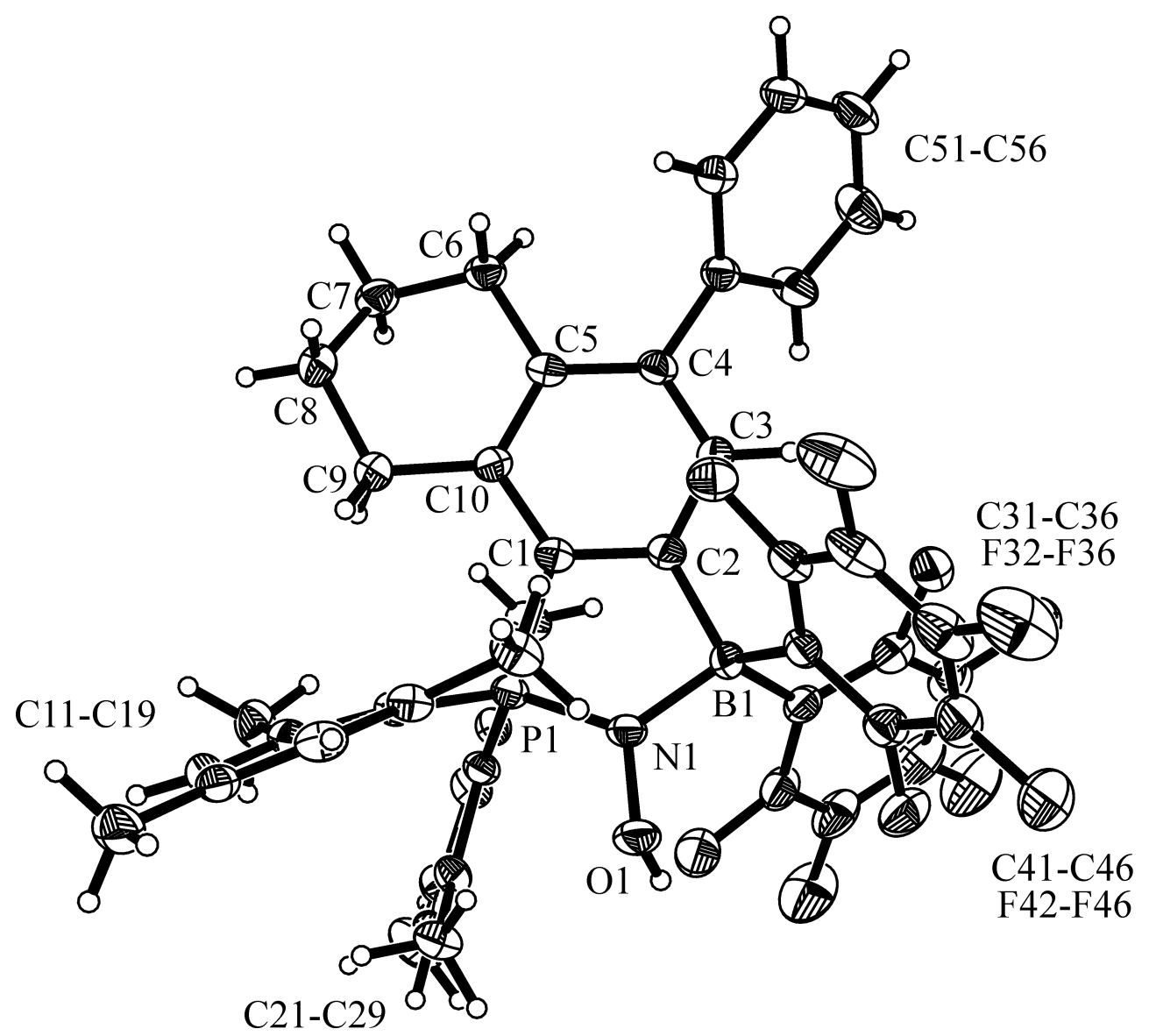

Figure S77: X-ray crystal structure of compound 22 (thermal ellipsoids are shown at the $30 \%$ probability level) 


\section{Solid State NMR Data}

\section{Experimental}

Solid State NMR measurements were carried out on a BRUKER Avance III 300 spectrometer (magnetic field strength of $7.05 \mathrm{~T}$ ). Magic angle spinning was used in 4mm NMR double and triple resonance probes. For ${ }^{11} \mathrm{~B}\left\{{ }^{1} \mathrm{H}\right\}$ MAS NMR experiments a short duration pulse of $0.5-1 \mu$ s was used corresponding approximately to a $30^{\circ}$ flip angle to achieve uniform excitation. A spinning speed of $12.0 \mathrm{kHz}$ and relaxation delays of 20-80 s were used. Chemical shifts are reported relative to an external sample of $\mathrm{BF}_{3} \cdot \mathrm{Et}_{2} \mathrm{O} .{ }^{31} \mathrm{P}\left\{{ }^{1} \mathrm{H}\right\}$ CPMAS NMR spectra were obtained at $12.5 \mathrm{kHz}$ spinning rate using proton $\pi / 2-$ pulses between 5 and $7 \mu \mathrm{s}$, a contact time of 3 to $5 \mathrm{~ms}$ with a ramp (90-100\% of the maximum power on the ${ }^{1} \mathrm{H}$ channel). The relaxation delay was 5-15 s. Spectra were externally referenced to $85 \% \mathrm{H}_{3} \mathrm{PO}_{4}$. TPPM-15 proton decoupling was conducted during acquisition of ${ }^{11} \mathrm{~B}$ and ${ }^{31} \mathrm{P}$ spectra with pulses of about 5.5-8 $\mu$ s length, corresponding to $10 / 12 \pi$-pulses. Spectral deconvolution was done with DMFIT software (version 2011). ${ }^{[1]}$ 

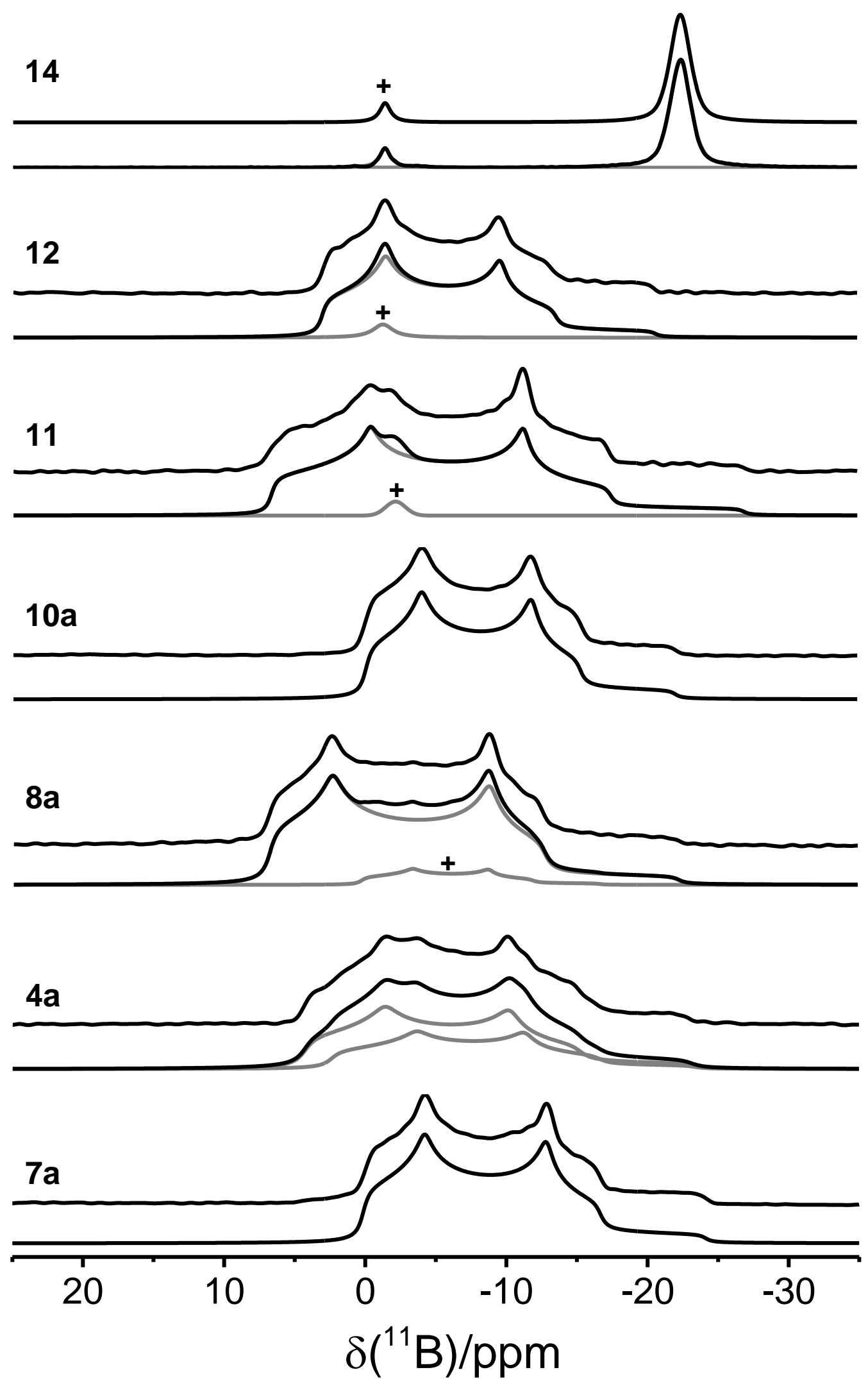

Figure S78: Experimental (top line) and simulated (bottom line) ${ }^{11} \mathrm{~B}$ MAS spectra of acyclic to cyclic to aromatic FLP (7a-4a-8a and 10a-11-12) and FLP-H $\mathrm{H}_{2}$ adduct 14 measured at a field of $7.05 \mathrm{~T}$ and a spinning speed of $12 \mathrm{kHz}$. Grey lines show simulated sub-spectra. Crosses mark impurities of the samples. 
Table S1: Experimental ${ }^{11} \mathrm{~B}$ chemical shift and nuclear electric quadrupole coupling parameters obtained via deconvolution of MAS spectra (Figure S78).

\begin{tabular}{|l|c|c|c|}
\hline Comp. & $\boldsymbol{\delta}_{\text {CS }}^{\text {iso }} / \mathbf{p p m}$ & $\mathbf{C}_{\mathrm{Q}} / \mathbf{M H z}$ & $\eta_{\mathrm{Q}}$ \\
\hline 7a & 2.0 & 1.95 & 0.30 \\
\hline $\mathbf{4 a}$ & $6.0 / 2.8$ & $2.05 / 1.92$ & 0.35 \\
\hline $\mathbf{8 a}$ & 9.3 & 2.15 & 0.24 \\
\hline $10 \mathrm{a}$ & 1.6 & 1.86 & 0.30 \\
\hline 11 & 8.8 & 2.28 & 0.35 \\
\hline 12 & 4.7 & 1.92 & 0.32 \\
\hline 14 & -21.8 & ca. $0.5 \mathrm{MHz}$ & --- \\
\hline
\end{tabular}

14
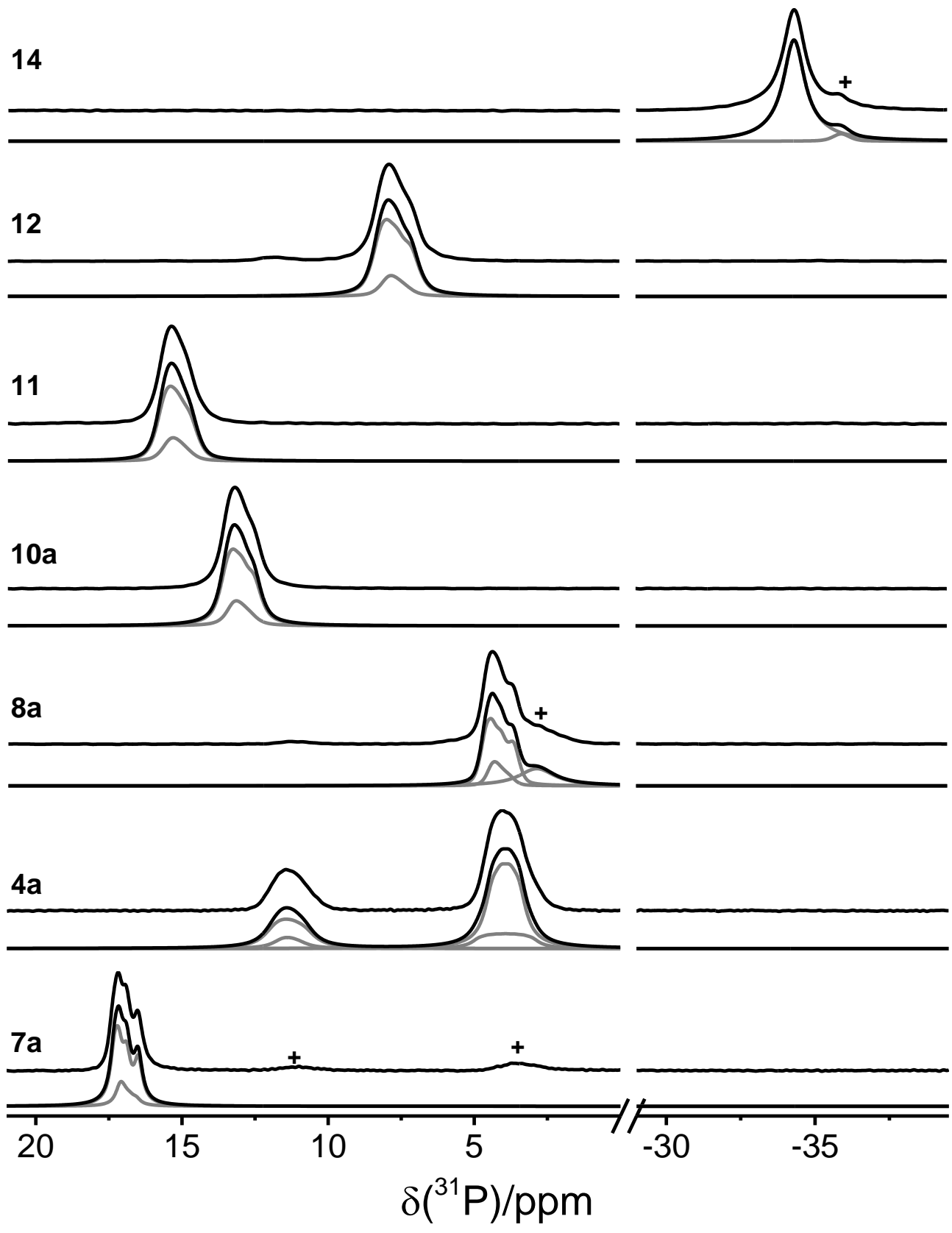

Figure S79: Experimental (top line) and simulated (bottom line) ${ }^{31} \mathrm{P}$ CPMAS spectra of acyclic to cyclic to aromatic FLP (7a-4a-8a and 10a-11-12) and FLP-H ${ }_{2}$ adduct 14 measured at a field strength of $7.05 \mathrm{~T}$ and a spinning speed of $12.5 \mathrm{kHz}$ using a 5 ms contact pulse for compounds $4 \mathrm{a}-12$ and a $3 \mathrm{~ms}$ contact pulse for compound 14. Grey lines show simulated sub-spectra for the ${ }^{11} \mathrm{~B}$ and ${ }^{10} \mathrm{~B}$ isotopologues. Crosses mark impurities of the samples. 
Table S2 : Experimental ${ }^{31} \mathrm{P}$ chemical shift and J-coupling constants obtained via deconvolution of CPMAS spectra (Figure S79).

\begin{tabular}{|c|c|c|}
\hline Comp. & $\boldsymbol{\delta}_{\mathbf{C S}}^{\text {iso }} \mathbf{~ p p m}$ & $\mathbf{J}\left({ }^{\mathbf{3 1}} \mathbf{P}-{ }^{11} \mathbf{B}\right) / \mathbf{H z}$ \\
\hline $\mathbf{7 a}$ & 17.0 & 33.0 \\
\hline $\mathbf{4 a}$ & $3.9 / 11.3$ & 39.0 \\
\hline $\mathbf{8 a}$ & 4.4 & 39.0 \\
\hline $\mathbf{1 0 a}$ & 13.1 & 38.0 \\
\hline $\mathbf{1 1}$ & 15.3 & 37.0 \\
\hline $\mathbf{1 2}$ & $\mathbf{7 . 8}$ & 44.0 \\
\hline $\mathbf{1 5}$ & -34.3 & --- \\
\hline
\end{tabular}

\section{Literature:}

[1] D. Massiot, F. Fayon, M. Capron, I. King, S. Le Calvé, B. Alonso, J.-O. Durand, B. Bujoli, Z. Gan and G. Hoatson, Magn. Reson. Chem. 2002, 40, 70-76. 


\section{EPR Data}

The EPR measurements were performed in quartz tubes. Solution EPR spectra were recorded on a JEOL continuous wave spectrometer JES-FA200 equipped with an X-band Gunn oscillator bridge and a cylindrical mode cavity. For all samples, a modulation frequency of 100 $\mathrm{kHz}$ was employed. All spectra were obtained from freshly prepared fluorobenzene solutions $(0.005 \mathrm{M})$ at $298 \mathrm{~K}$.

Spectral simulation was performed using the program QCMP 136 by Prof. Dr. Frank Neese from the Quantum Chemistry Program Exchange as used by Neese et al. in J. Am. Chem. Soc. 1996, 118, 8692-8699. The fittings were performed by the "chi by eye" approach. Collinear $g$ and $A$ tensors were used. Coupling to ${ }^{10} \mathrm{~B}(I=3 ; 19.9 \%$ abundant $)$ was neglected in all simulations.
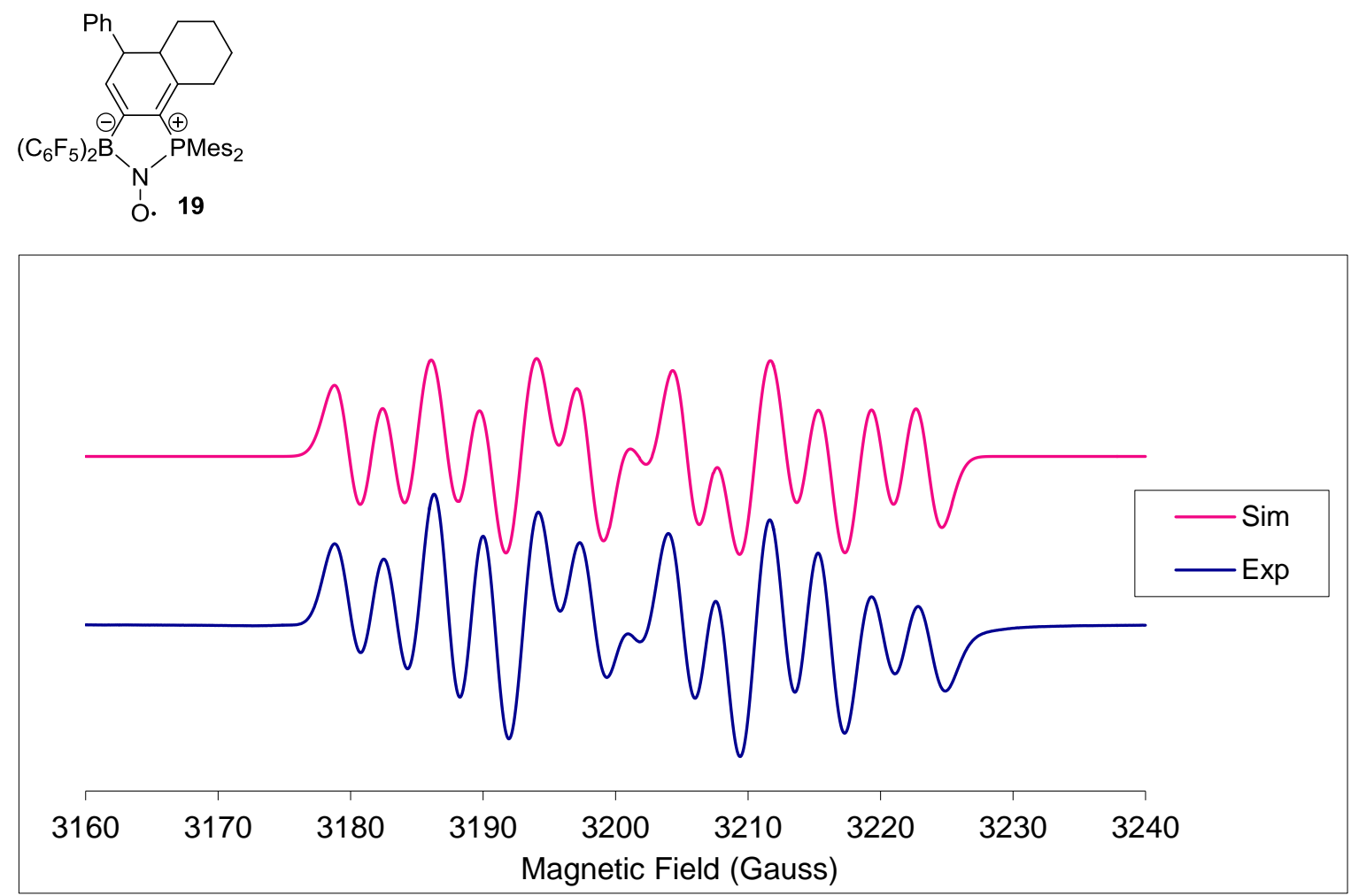

Figure S80: X-band EPR spectrum and simulation for compound 19 (fluorobenzene, RT): $8.986159 \mathrm{GHz}$, ModWidth $=0.1 \mathrm{mT}$, Power $=1.0 \mathrm{~mW}$, time constant $=0.1 \mathrm{~s}$. Simulation (Gaussian lineshape with $1.3 \mathrm{G}$ linebroadening) gives giso $=2.0053, A\left({ }^{14} \mathrm{~N}\right)=22.2 \mathrm{MHz}, A\left({ }^{31} \mathrm{P}\right)=49.6 \mathrm{MHz}, A\left({ }^{11} \mathrm{~B}\right)=9.5 \mathrm{MHz}$ 


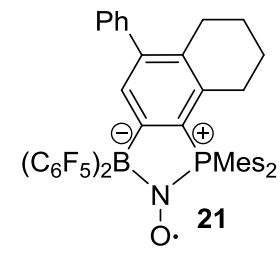

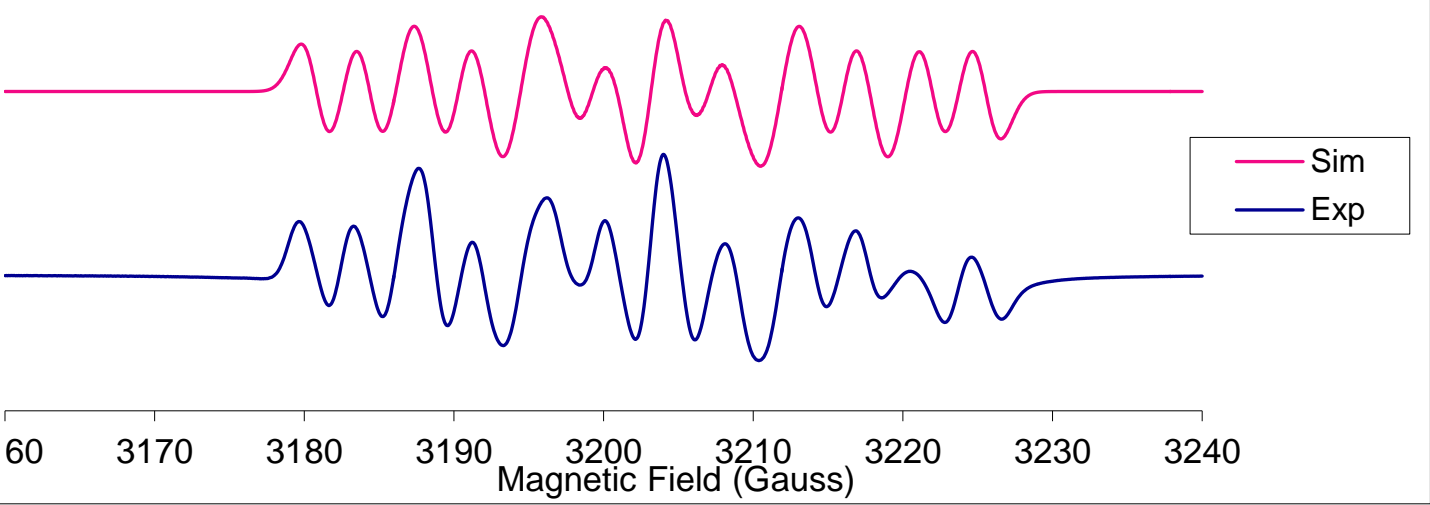

Figure S81: X-band EPR spectrum and simulation for compound 21 (fluorobenzene, RT): $8.989816 \mathrm{GHz}$, ModWidth $=0.1 \mathrm{mT}$, Power $=1.0 \mathrm{~mW}$, time constant $=0.03 \mathrm{~s}$. Simulation (Gaussian lineshape with $1.2 \mathrm{G}$ linebroadening) gives giso $=2.0052, A\left({ }^{14} \mathrm{~N}\right)=23.3 \mathrm{MHz}, A\left({ }^{31} \mathrm{P}\right)=48.9 \mathrm{MHz}, A\left({ }^{11} \mathrm{~B}\right)=10.0 \mathrm{MHz}$ 